THERMO-MECHANICALLY TUNABLE BRAGG GRATING FILTERS ON SILICON-ON-INSULATOR RIB WAVEGUIDE BRIDGES

by

Christopher R. Raum

B. A. Sc. (Regina), M. A. Sc. (Regina)

A Thesis

Submitted to the Faculty of Graduate Studies and Research in Partial Fulfilment of the Requirements for the Degree of Doctor of Philosophy

Ottawa-Carleton Institute for Electrical Engineering

Department of Electronics

Carleton University

Ottawa, Canada

Copyright $(C$ August 2010 by Christopher R. Raum 
Library and Archives
Canada

Published Heritage Branch

395 Wellington Street Ottawa ON K1A ON4 Canada
Bibliothèque et

Archives Canada

Direction du

Patrimoine de l'édition

395, rue Wellington

Ottawa ON K1A ON4

Canada
Your file Votre référence
ISBN: $978-0-494-70555-1$
Our file Notre référence
ISBN: $978-0-494-70555-1$
NOTICE:

The author has granted a nonexclusive license allowing Library and Archives Canada to reproduce, publish, archive, preserve, conserve, communicate to the public by telecommunication or on the Internet, loan, distribute and sell theses worldwide, for commercial or noncommercial purposes, in microform paper, electronic and/or any other formats.

The author retains copyright ownership and moral rights in this thesis. Neither the thesis nor substantial extracts from it may be printed or otherwise reproduced without the author's permission.
AVIS:

L'auteur a accordé une licence non exclusive permettant à la Bibliothèque et Archives Canada de reproduire, publier, archiver, sauvegarder, conserver, transmettre au public par télécommunication ou par l'Internet, prêter distribuer et vendre des thèses partout dans le monde, à des fins commerciales ou autres, sur support microforme, papier, électronique et/ou autres formats.

L'auteur conserve la propriété du droit d'auteur et des droits moraux qui protège cette thèse. $\mathrm{Ni}$ la thèse ni des extraits substantiels de celle-ci ne doivent être imprimés ou autrement reproduits sans son autorisation.
In compliance with the Canadian Privacy Act some supporting forms may have been removed from this thesis.

While these forms may be included in the document page count, their removal does not represent any loss of content from the thesis.
Conformément à la loi canadienne sur la protection de la vie privée, quelques formulaires secondaires ont été enlevés de cette thèse.

Bien que ces formulaires aient inclus dans la pagination, il n'y aura aucun contenu manquant. 
The undersigned recommend to the Faculty of Graduate

Studies and Research acceptance of the thesis

(Signatures on file at the Faculty of Graduate Studies and Research)

\title{
THERMO-MECHANICALLY TUNABLE BRAGG GRATING FILTERS ON SILICON-ON-INSULATOR RIB WAVEGUIDE BRIDGES
}

\author{
submitted by \\ Christopher R. Raum, B.A.Sc., M.A.Sc. \\ in partial fulfillment of the requirements for the degree of \\ Doctor of Philosophy in Electrical Engineering
}

Professor Niall Tait, Co-Supervisor

Professor Robert Gauthier, Co-Supervisor

Professor Qi-Jun Zhang, Chair, Department of Electronics

Professor Rafael Kleiman, External Examiner

Ottawa-Carleton Institute for Electrical Engineering

Department of Electronics

Carleton University

July 2010 


\begin{abstract}
This thesis explores the integration of an optical device within a micromechanical structure to enhance its performance and enable behaviour it would otherwise be incapable of. Thermo-mechanically tunable Bragg grating filters on silicon-on-insulator rib waveguide bridges have been designed, fabricated and characterised to demonstrate what happens when an optical device, and the actuator used to tune its optical response, are physically the same structure. The process flow developed to fabricate the device was a five mask process that included a bridge waveguide, integrated filter, and integrated heater. A surface micromachining technique was developed to release up to $4000 \mu \mathrm{m}$ long, $5 \mu \mathrm{m}$ thick waveguide bridges.

The device has three distinct operating regimes: pre-buckle, buckle, and postbuckle. The pre-buckle experimental thermal sensitivity of the filter was $76 \mathrm{pm} /{ }^{\circ} \mathrm{C}$ and the theoretical sensitivity was $83 \mathrm{pm} /{ }^{\circ} \mathrm{C}$. During the transient buckle regime, the Bragg filter wavelength was measured to shift $0.95 \mathrm{~nm}$, and theorised to shift $0.55 \mathrm{~nm}$. The post-buckle experimental thermal sensitivity of the filter was $88 \mathrm{pm} /{ }^{\circ} \mathrm{C}$ and the theoretical sensitivity was $99 \mathrm{pm} /{ }^{\circ} \mathrm{C}$.

The rib waveguide bridge was observed to possess a meta-stable regime between the pre- and post-buckle regimes. Before the critical buckle temperature could be attained, the bridge deflected $0.5 \mu \mathrm{m}$ out-of-plane and remained static over a range of $7.5{ }^{\circ} \mathrm{C}$, whereupon it deflected to its full $15.1 \mu \mathrm{m}$ buckling mode height. This metastable deflection caused a Bragg wavelength shift of $0.39 \mathrm{~nm}$. The thermal sensitivity of the Bragg filter wavelength in this meta-stable regime was $62 \mathrm{pm} /{ }^{\circ} \mathrm{C}$. Rectangular crosssection beams did not produce this behaviour.
\end{abstract}


Mechano-optical bi-stability was also observed. In this bi-stable regime there would be two possible Bragg wavelengths for a given temperature, depending on whether the device was in a forward or return path. The bi-stable regime occurred over a span of $15^{\circ} \mathrm{C}$. The Bragg wavelength difference at the start of the post-buckle regime was $0.95 \mathrm{~nm}$, while the Bragg wavelength difference at the return to the pre-buckle regime was $0.77 \mathrm{~nm}$. 


\section{Acknowledgements}

Composing this thesis has been a process that drew upon many elements of engineering and science. As such, I had support from a variety of sources for education, advice, or just reassurance that I would like to acknowledge. First and foremost I would like to thank my supervisors, Professors Niall Tait and Robert Gauthier. Although they advised me in their areas of expertise throughout my research, I would also like to acknowledge their trust in the beginning that I could achieve what I proposed; and their patience later on while I pulled all the threads together into the tapestry that would become my thesis.

Next I would like to thank my mom and dad, Elizabeth and Richard Raum. As a symphonic composer and a professor of music, respectively, they nevertheless let me be the "black note" of the family. In my formative years they tolerated my inquisitive nature

and encouraged my love for science, the result of which carried me to the completion of my Ph.D. They, along with my grandmother, Elizabeth Hodges; my uncle, Kenny Raum; and my sisters, Jessica and Erika gave me emotional support and more when I doubted myself. Thanks, too, to my brother-in-law, Marc Lafoy, who performed the beautiful graphic art of the post-buckle device configuration figure.

Early on when I didn't quite know what I was getting myself into, Professors Henry Schriemer and André Lalonde, and Drs. Peter Krug and Stewart Clark, gave invaluable guidance. In Bragg theory and optical characterisation, Professor Jacques Albert was a constant source of knowledge and experience. In mechanical theory, Professor Heng-Aik Khoo helped me understand the nature of the beams I had fabricated.

In fabrication I received engineering assistance from Professor Garry Tarr and Dean Ducharme. Help with technical and operator expertise came, with great patience, 
from Rob Vandusen, Carol Adams, and Rick Adams. All these people taught me and enhanced my skills in the operation of the equipment, as well as my knowledge of the materials, used to fabricate my device. Without their assistance I could not have made the device I did.

At National Research Council Canada, Edith Post helped me optically characterise my first device to help confirm I was on the right track, and Dr. Jean Lapointe processed the Bragg grating mask on my final device. A special thanks goes to Jean for his patience with me in working through the initial scaling mix-ups that plagued the grating write process. Thanks also goes out to Scott Newman, who helped me program the lab equipment for data capture; and the support staff at the department of electronics, especially Blazenka Powers and Anna Lee.

And last, but certainly not least, there was Khaled Mnaymneh, a fellow Ph.D. student and devotee of natural science. His friendship and great sense of humour kept me moving forward throughout my research. Our conversations were a whetstone to hone my ideas against. Of all the people who helped me arrive at the finish line, Khaled, you were my greatest booster.

Financial assistance was provided by the Canadian Institute for Photonic Innovations (CIPI) and Carleton University’s scholarship fund.

Know your limits, but never accept them. 


\section{Table of Contents}

1 Introduction

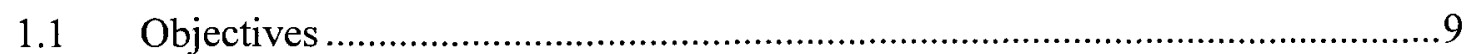

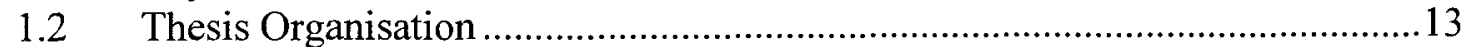

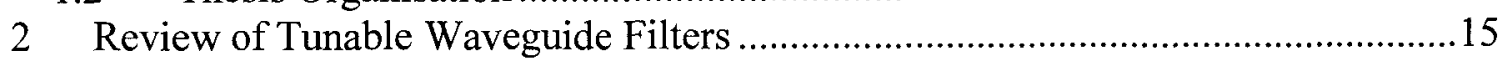

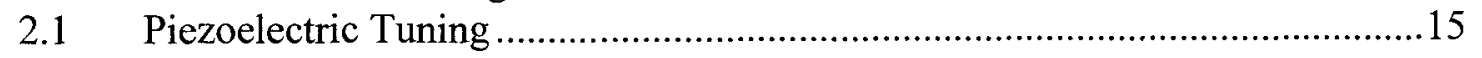

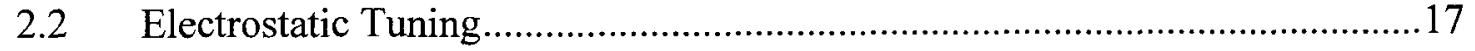

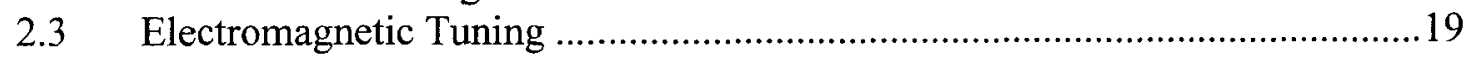

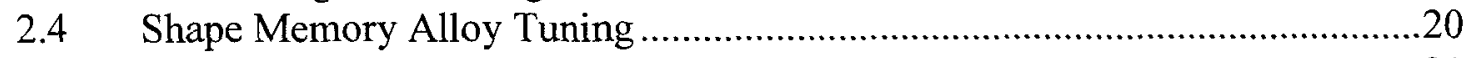

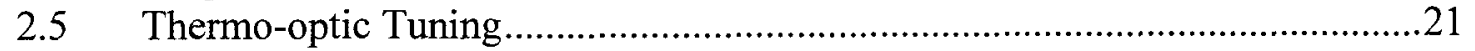

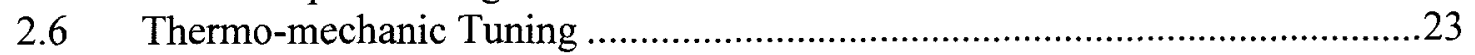

3 A Method for Thermally Tuning the Wavelength of an Optical Filter ....................25

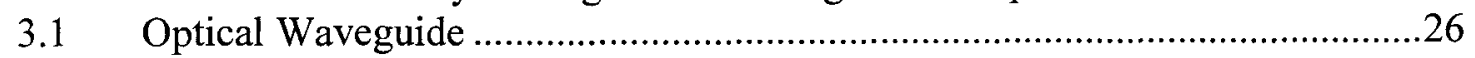

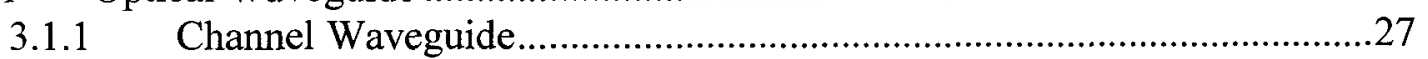

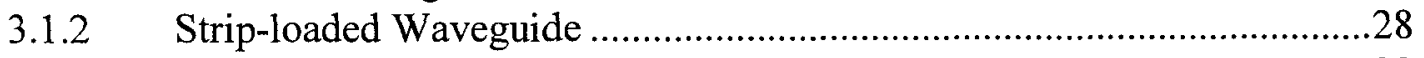

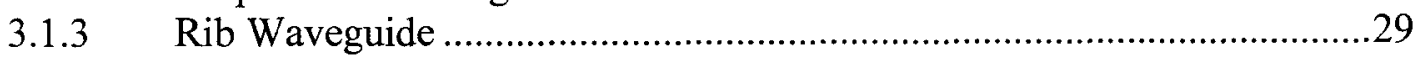

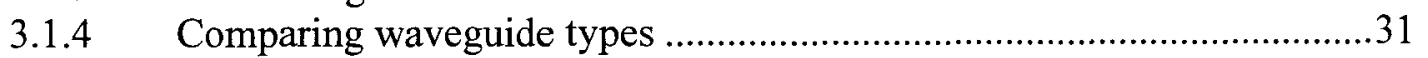

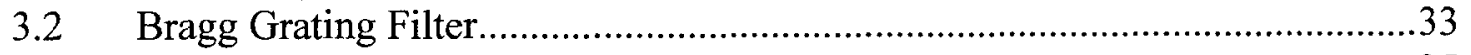

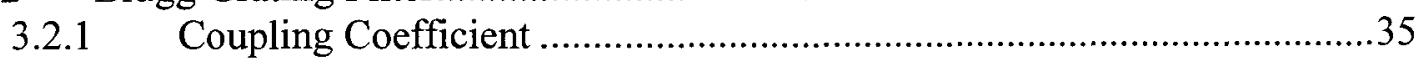

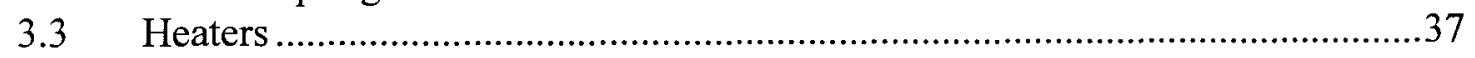

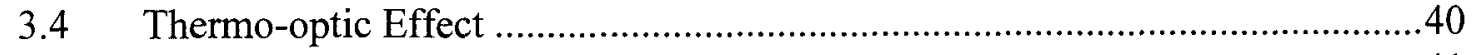

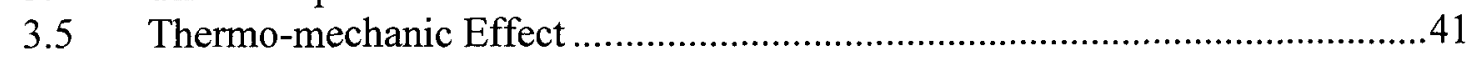

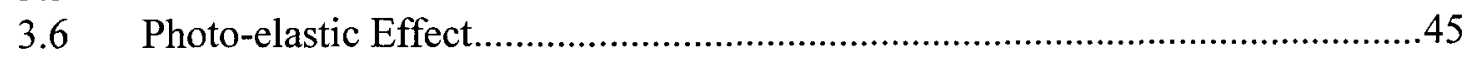

3.7 Thermo-mechanical axial beam deformation ................................................49

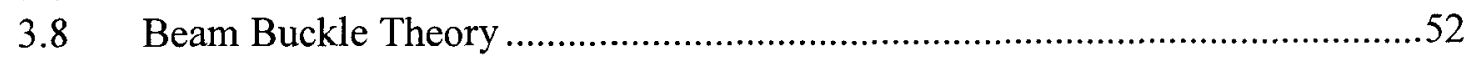

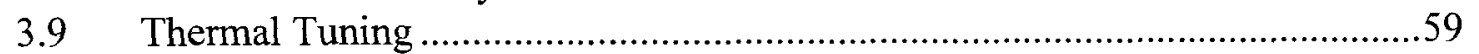

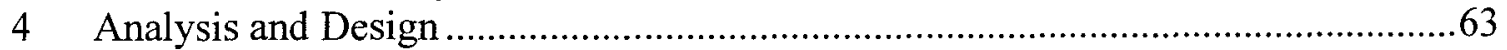

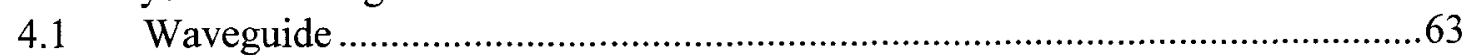

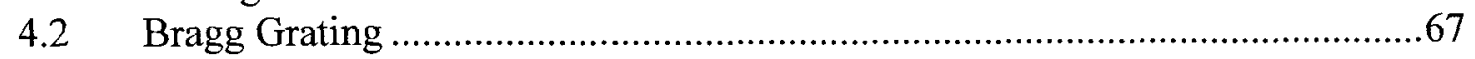

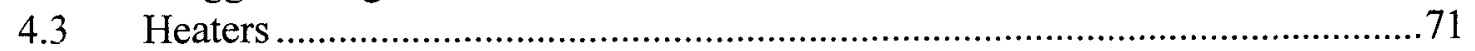

4.4 Thermally Tuning the Bragg Filter Wavelength ............................................ 73

4.4.1 Analysis of Bragg Wavelength Shift in the Pre-Buckle Regime................74

4.4.2 Analysis of Bragg Wavelength Shift in the Buckle Regime .....................77

4.4.3 Analysis of Bragg Wavelength Shift in the Post-Buckle Regime ..............80

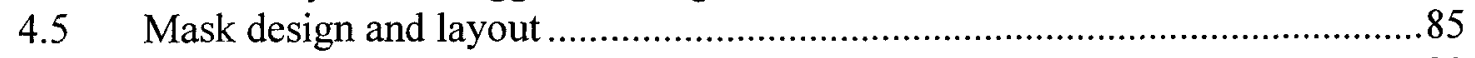

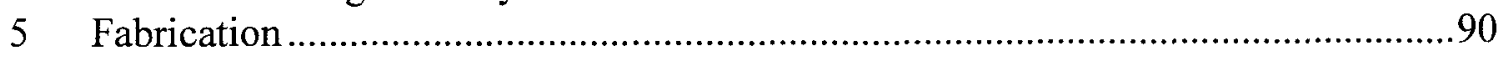

5.1 Mask Generation and Lithography ....................................................... 93

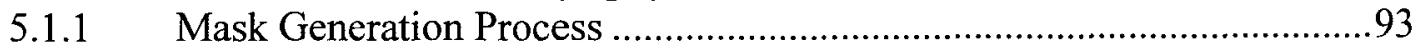

5.1.2 Electron Beam Lithography ...............................................................98

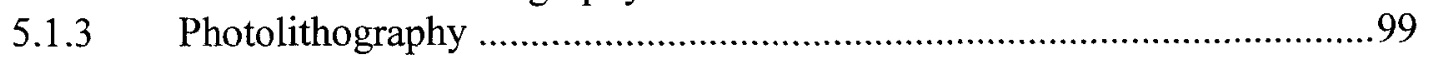

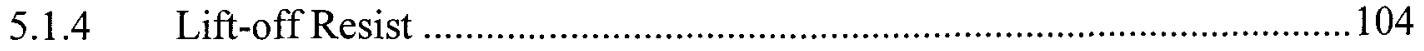

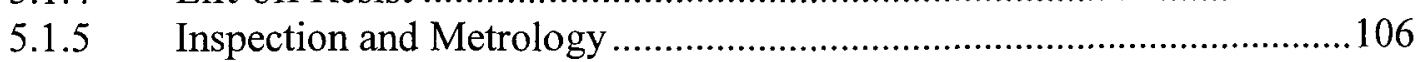

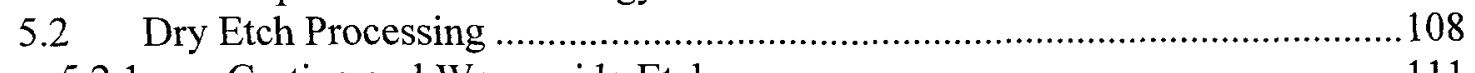

5.2.1 Grating and Waveguide Etch....................................................... 111 


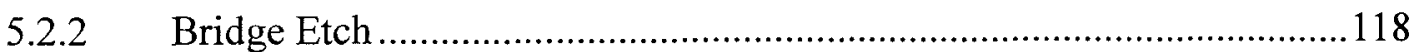

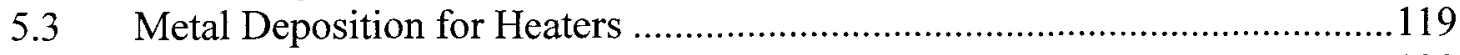

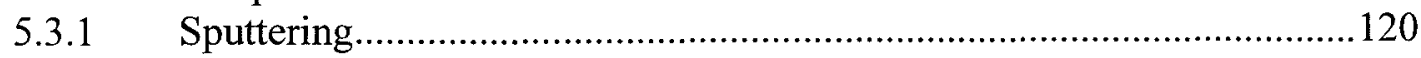

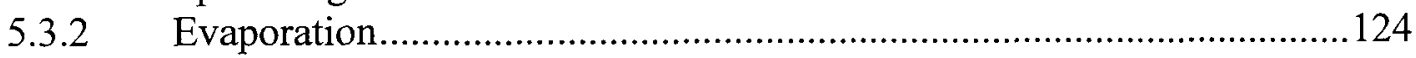

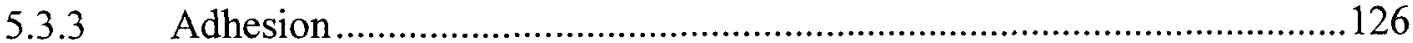

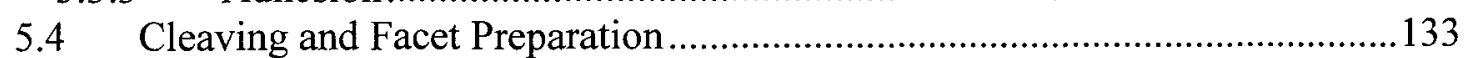

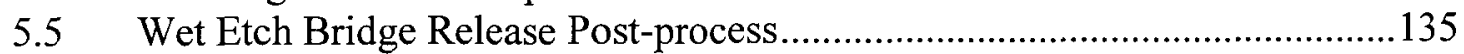

5.5.1 Bridge Release Process Development ...................................................136

5.5.2 Oxygen Plasma Dry Release with PR support .......................................141

5.5.3 Structural Release Shock and Etch Profile ...........................................150

5.6 Packaging and Wirebonding ...................................................................152

5.7 Device Die Completed for Test and Characterisation .....................................156

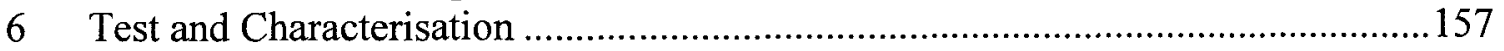

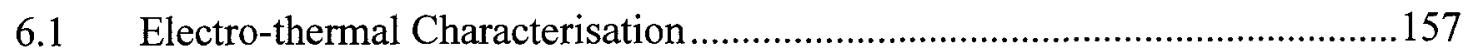

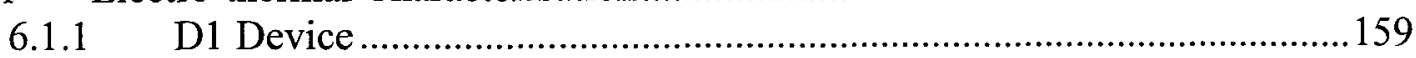

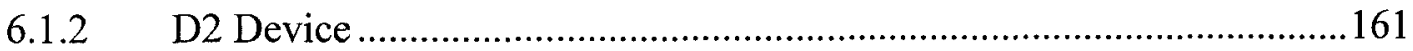

6.2 Thermo-mechanical Characterisation ......................................................163

6.3 Thermo-optical and Thermomechano-optical Characterisation .......................177

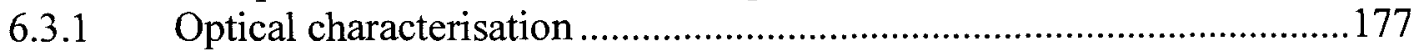

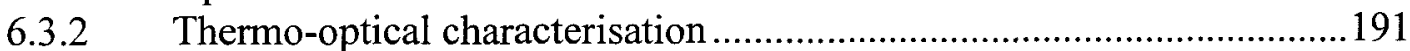

6.3.3 Thermomechano-optical characterisation........................................... 198

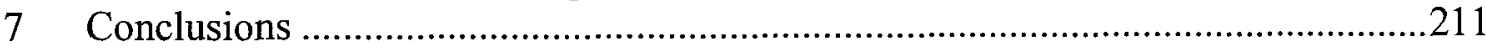

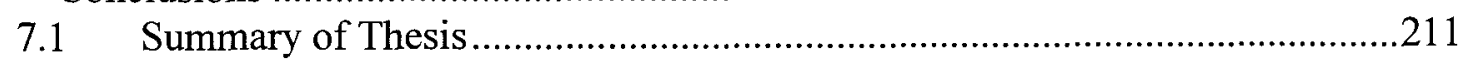

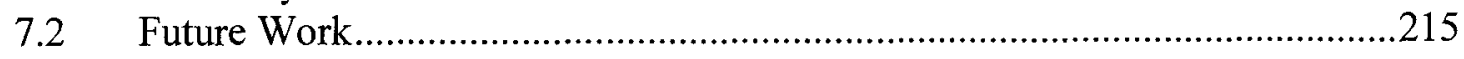

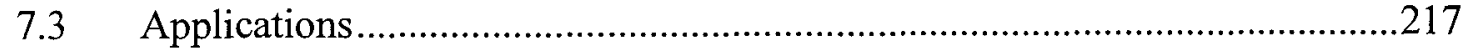

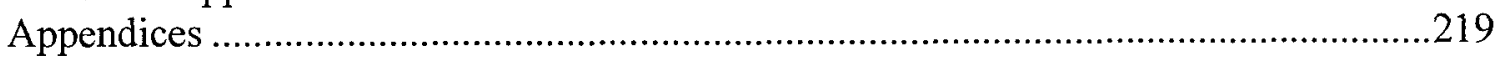

Appendix I: Verifying Critical Buckle Stress Calculations.....................................219

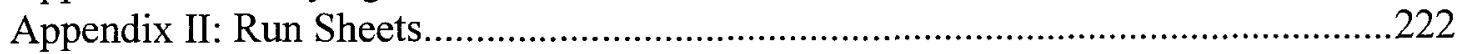

Appendix III: Alternative Processes for Heater Protection during Bridge Release Postprocess 


\section{List of Tables}

TABLE 1: THERMO-OPTIC PROPERTIES OF MATERIALS USED IN INTEGRATED OPTICS...................................21 TABLE 2: SUMMARY OF WAVEGUIDE DESIGNS WITH PROCESS CHALLENGES, STRENGTHS AND WEAKNESSES.

32

TABLE 3. LIST OF CRITICAL WAVEGUIDE BRIDGE STATES THAT CORRESPOND TO FIG. 3.13, WITH

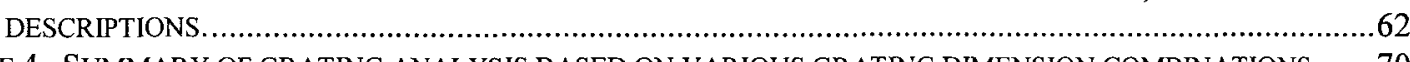

TABLE 4. SUMMARY OF GRATING ANALYSIS BASED ON VARIOUS GRATING DIMENSION COMBINATIONS .......70

TABLE 5. SUMMARY OF DEVICE VARIANTS FOR CHARACTERISATION ....................................................89

TABLE 6. COMPARISON OF ETCH STABILITY AS A FUNCTION OF BOE PRE-ETCH DIP..................................118

TABLE 7. SUMMARY OF DEVICE DIE USED IN TEST AND CHARACTERISATION EXPERIMENTS. UNFILLED BOXES INDICATE THAT THE DEVICE WAS NOT PROCESSED WITH THE MATERIAL OR STRUCTURE SHOWN IN THAT

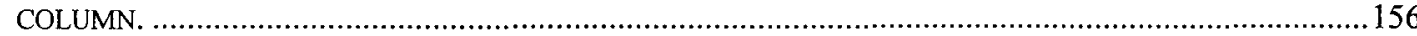

TABLE 8. COMPARISON OF THEORETICAL HEATER RESISTANCE TO EXPERIMENTAL MEASUREMENT. ...........158

TABLE 9. COMPARISON OF RESISTANCE DUE TO OHMIC CONTACT ....................................................158 


\section{List of Figures}

FIGURE 1.1. DEFLECTION OF SILICON MICROMACHINED CANTILEVER TO DEMONSTRATE ITS MECHANICAL PROPERTIES [11].

FIGURE 1.2. ANISOTROPIC WET ETCH OF $(110) /(111)$ SILICON AT $55^{\circ}$ FROM THE (100) FLAT. THE TRENCHES DEFINE A RIB WAVEGUIDE [12]

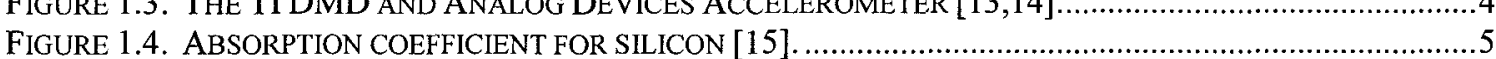

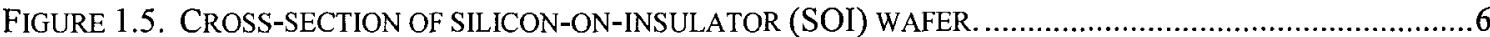

FIGURE 1.6. EXAMPLES OF LARGE ACTUATOR STRUCTURES USED TO TUNE SMALL ENABLING COMPONENTS $[16,17] \ldots$

FIGURE 1.7. BUCKLE BEAM THERMAL ACTUATOR TO DEFLECT MIRROR [18] .

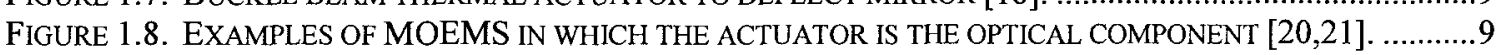

FIGURE 1.9. BRAGG GRATING FILTER ON SOI RIB WAVEGUIDE IN PRE-BUCKLE CONFIGURATION.................12

FIGURE 1.10. CROSS-SECTION OF BRAGG GRATING FILTER ON SOI RIB WAVEGUIDE BRIDGE IN BUCKLE MODE CONFIGURATION. THE VERTICAL DEFLECTION HAS BEEN EXAGGERATED TO SHOW ITS SHAPE [22] ....113

FIGURE 2.1: PIEZOELECTRIC AXIAL COMPRESSION OF FIBRE BRAGG GRATING [23] ...............................16

FIGURE 2.2. MEMS STRUCTURE - DBR HOUSED IN A TORSIONAL ARM CANTILEVER [31] ..........................17

FIGURE 2.3: OVER-AND-UNDER BRIDGE FORMS FABRY - PEROT CAVITY [32] ........................................... 18

FIGURE 2.4: WAVELENGTH ADD-DROP SWITCHING USING TILTING MICRO-MIRRORS [33]..........................19

FIGURE 2.5. COMMERCIAL EXAMPLE OF AN OXC; LUCENT'S "LAMBDA ROUTER" [34].............................19

FIGURE 2.6. ELECTROMAGNETICALLY TUNABLE IR FILTER [36] .....................................................20

FIGURE 2.7: SCHEMATIC CONFIGURATION OF A SMA TUNABLE FBG FILTER [40] .................................21

FIGURE 2.8: IN-PLANE SILICON TOF HORIZONTALLY ALIGNED WITH AN OPTICAL FIBRE [46]...................22

FIGURE 2.9: SCHEMATIC OF THERMO-ELASTICALL Y PITCH-TUNABLE GRATING FILTER [48] ......................24

FIGURE 2.10. SCHEMATIC OF A THERMALLY INSENSITIVE CANTILEVER BASED VERTICAL CAVITY FILTER TUNED BY THERMAL EXPANSION [49].

FIGURE 3.1. CHANNEL WAVEGUIDE EXAMPLE.

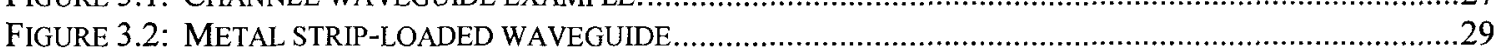

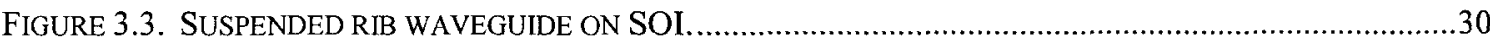

FIGURE 3.4. TOP VIEW AND CROSS-SECTION OF A RIB WAVEGUIDE FOR EIM. ..........................................32

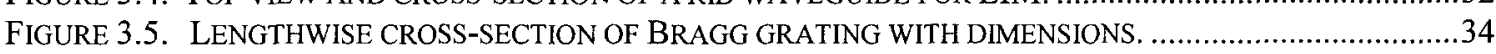

FIGURE 3.6. DIMENSIONS OF A GRATING USED FOR THE COUPLED MODE SOLUTION OF THE BRAGG FILTER

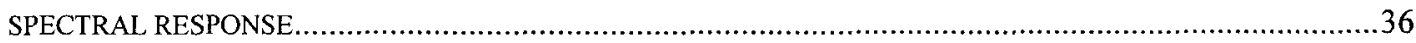

FIGURE 3.7. ORIENTATION OF WAVEGUIDE BRIDGE WITHIN THE CRYSTALLOGRAPHIC PLANES OF SINGLE CRYSTAL SILICON TO HELP DETERMINE THE MECHANICAL PROPERTIES OF THE BRIDGE [68]..............44

FIGURE 3.8. CUBIC HEXOCTAHEDRAL SILICON SHOWING THE ALIGNMENT OF THE OPTICAL AND CRYSTALLOGRAPHIC AXIS IN AN OPTICAL ORIENTATION PLOT, ................................................45

FIGURE 3.9. ISOTROPIC OPTICAL INDICATRIX FOR A CUBIC HEXOCTAHEDRAL CRYSTAL SUCH AS SILICON ...46

FIGURE 3.10. LONG SLENDER FIXED-FIXED BEAM SUBJECT TO A UNIFORM TEMPERATURE INCREASE. ..........49

FIGURE 3.11. THE BUCKLING MODE SHAPE OF A FIXED-FIXED BEAM WHERE THE $W$-AXIS IS EXAGGERATED TO SHOW THE VERTICAL DISPLACEMENT, $\delta$, OF THE BEAM................................................................5

FIGURE 3.12. MOMENT OF INERTIA DIAGRAM OF RIB WAVEGUIDE CROSS-SECTION .................................55

FIGURE 3.13: IMPORTANT STATES FOR THE WAVEGUIDE BRIDGE FILTER OVER PRE-BUCKLE TO POST-BUCKLE REGIMES, SHOWING THE BRAGG WAVELENGTH VERSUS THE WAVEGUIDE BRIDGE TEMPERATURE [AFTER 86].

FIGURE 4.1. CROSS-SECTION OF AN SOI RIB WAVEGUIDE WITH PRINCIPAL DIMENSIONS MARKED. THE

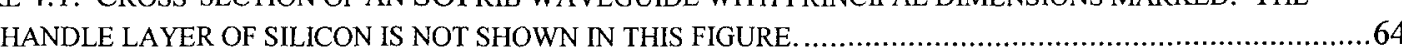

FIGURE 4.2. W/H AS A FUNCTION OF H/H FOR THE SINGLE MODE PROPAGATION LIMIT [87] ......................65

FIGURE 4.3: SINGLE MODE PROFILE OF SUSPENDED RIB WAVEGUIDE WITH $H=5.0 \mu \mathrm{M}, W=5.0 \mu \mathrm{M}$, AND $H=$ $890 \mathrm{NM}$

FIGURE 4.4: REFLECTIVITY OF THE BRAGG FILTER AS A FUNCTION OF WAVELENGTH DEVIATION FROM THE BRAGG CONDITION WITH $A=200$ NM AND $L_{B}=500 \mu \mathrm{M}$ 
FIGURE 4.5. REFLECTIVITY FOR DIFFERENT GRATING DEPTHS WITH A GRATING LENGTH $L_{B}=1000 \mathrm{MM} \ldots \ldots .70$ FIGURE 4.6. REFLECTIVITY FOR DIFFERENT GRATING LENGTHS WITH A GRATING DEPTH $A=100$ NM...........71

FIGURE 4.7. EXAMPLES OF TENSILE, COMPRESSIVE AND NEUTRAL STRESS ON MICROMACHINED $\mathrm{SIO}_{2}$ CANTILEVERS [93]....

FIGURE 4.8. TWO $4000 \mu \mathrm{M}$ LONG, $5 \mu \mathrm{M}$ THICK WAVEGUIDE BRIDGES AT ROOM TEMPERATURE. BRIDGE A SHOWS NO RESIDUAL STRESS. BRIDGE B SHOWS BI-STABLE COMPRESSIVE RESIDUAL STRESS..............82

FIGURE 4.9. LOCATION OF CENTROID, $Y$, IN A RIB WAVEGUIDE BRIDGE OF SPECIFIC DIMENSIONS.................83

FIGURE 4.10. LENGTHWISE CROSS-SECTION OF BRIDGE WAVEGUIDE IN PRE- AND POST-BUCKLE CONFIGURATIONS WITH PRE-BUCKLE AND POST-BUCKLE AXIAL BENDING STRESS DISTRIBUTIONS.......83

FIGURE 4.11. THEORETICAL PLOT OF THERMOMECHANO-OPTICAL DEVICE BEHAVIOUR AS A RESULT OF ANALYSIS OVER THE PRE-BUCKLE, BUCKLE, AND POST-BUCKLE REGIMES. .....................................8

FIGURE 4.12. PARTIAL D1 MASK SET INCLUDING THE HEATER MASK AND BRIDGE MASK..........................86

FIGURE 4.13. ALIGNMENT WINDOWS TO ASSIST IN CENTRING THE $2000 \mu \mathrm{M}$ AND $4000 \mu \mathrm{M}$ LONG BRIDGE AND HEATER MASKS ALONG THE PC GRATING MASK IN DEVICE D1 ....................................................8

FIGURE 4.14. D2 MASK SET INCLUDING THE BRAGG GRATING, RIB WAVEGUIDE, BRIDGE, AND HEATER MASKS FOR $500 \mu \mathrm{M}, 1000 \mu \mathrm{M}, 2000 \mu \mathrm{M}$, AND $4000 \mu \mathrm{M}$ BRIDGE LENGTHS..............................................8

FIGURE 4.15. THE $500 \mu \mathrm{M}$ BRIDGE SET SHOWING DEVICE VARIATIONS .................................................8

FIGURE 5.1. PROCESS FLOW DIAGRAM FOR D2 FABRICATION STREAM.................................................92

FIGURE 5.2. GCA MANN 1600A PATTERN GENERATOR ..............................................................94

FIGURE 5.3. CLEAR FIELD D2 HEATER MASK FROM PG COMPARED TO FINAL PHOTOREDUCED DARK FIELD MASK WITH A DIME FOR SCALE.

FIGURE 5.4. THE EXPOSURE PATH IS ALONG THE X-AXIS. ROTATION OF THE HEATER MASK WITHIN THE MASK FILE ALLOWED A SINGLE STRIP TO BE EXPOSED IN A SINGLE PASS ALONG THE X-AXIS SO THAT CUMULATIVE EXPOSURE ALIGNMENT ERROR WOULD NOT OCCUR. .............................................96

FIGURE 5.5. ORIEL 8410 PHOTOMASK CONTACT PRINTER AND JADE 4M- 10AXYL S\&R CAMERA.............97

FIGURE 5.6. COMPARISON OF THE MASK LAYOUT FILE, TO DARK FIELD MASK, TO GOLD LIFT-OFF PATTERN

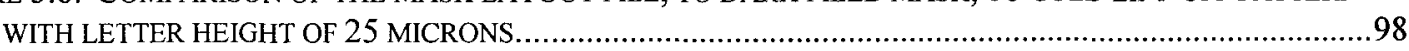

FIGURE 5.7. ALKALINE AND ACIDIC WET BENCHES IN PHOTOLITHOGRAPHY (YELLOW ROOM) ....................99

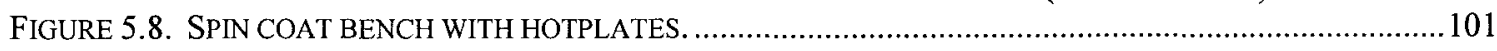

FIGURE 5.9. SINGLE HOLE VACUUM CHUCK FOR DIE AS SMALL AS 6 MM SQUARE.................................... 101

FIGURE 5.10. KARL-SUSS MA6 CONTACT ALIGNER ............................................................................. 103

FIGURE 5.11. CROSS-SECTION DIAGRAM OF LIFT-OFF RESIST PROCESS FOR HEATER STRIP OR BOND PAD ...105

FIGURE 5.12. EXAMPLE OF OVERHANG OF PR ON LOR WITH D1 DEVICE BOND PAD LITHOGRAPHY. ..........106

FIGURE 5.13. THE TENCOR P1 PROFILOMETER AND NANOSPEC 3000 REFLECTOMETER............................108

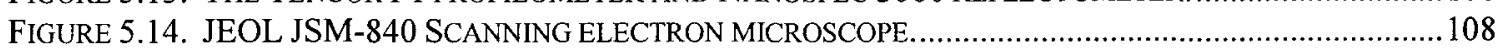

Figure 5.15. PlaSMATIC SYSTEMS PlaSMA PREEN II PLASMA ETCHER/CLEANER ...................................110

FIGURE 5.16. TECHNICS PLANAR ETCH II PLASMA ETCHER USED PRIMARILY FOR DESCUMMING...............111

FIGURE 5.17. MAIN ETCH CONTROL ELEMENTS OF AN ECR RIE [AFTER 96] ...........................................112

FIGURE 5.18. THE CARLETON UNIVERSITY MFL PLASMATHERM SLR-772 ECR RIE. ..........................114

FIGURE 5.19. SILICON GRASS FROM THE A) GRATING ETCH, AND B) AND C) WAVEGUIDE ETCH, RESULTING FROM NON-IDEAL ETCH PARAMETERS.

FIGURE 5.20. PR TUNING THE ANISOTROPY OF THE ETCH REMOVED THE SILICON GRASS B) AND C), BUT

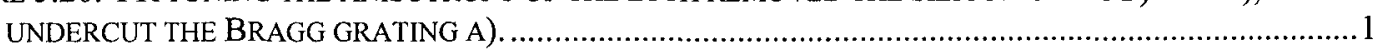

FIGURE 5.21. ORIGINAL GRATING AND WAVEGUIDE ETCH ON A) BARE BACKING WAFER, B) PR BALANCED BACKING WAFER, AND C) BARE BACKING WAFER WITH PROCESS IMPROVEMENTS AND ECR OPERATION

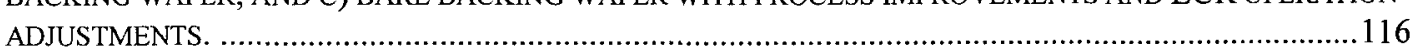

FIGURE 5.22. BRIDGE ETCH WITH ONE MICRON THICK BOX FOR THE D1 STREAM AND TWO MICRON THICK BOX FOR THE D2 STREAM, EACH REVEALING THEIR OXIDE INTERFERENCE COLOURS. ......................119

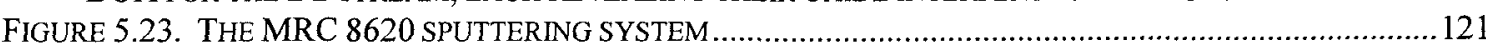

FIGURE 5.24. OVAL OUTLINE OF BOND WEDGE IN REMAINING GOLD BOND WIRE ON THE NICR SURFACE. ..123

FIGURE 5.25. NICR HEATER STRIP AND GOLD BOND PAD VIA SPUTTER DEPOSITION ...............................123

FIGURE 5.26. THE BALZERS BA510 EVAPORATION SYSTEM

FIGURE 5.27. GOLD HEATER STRIPS AND BOND PADS VIA EVAPORATION DEPOSITION .............................126

FIGURE 5.28. THE HF BRIDGE RELEASE ON D1 DIE CAUSED SILICON UNDER THE BOND PADS TO RUPTURE, ALLOWING THE BOND PADS ON THE DEVICE LAYER TO BREAK AWAY.... 
FIGURE 5.29. FRAGMENTED HEATER STRIPS DELAMINATE, WITH BOND PADS REMAINING IN PLACE ON D1 DEVICES......

FIGURE 5.30. DELAMINATION OF HEATER STRIPS WITH HEATERS REMAINING INTACT ON D2-4 DIE. INFILTRATION REPLACEMENT PR FOR BRIDGE RELEASE SUPPORT IS STILL IN PLACE (FOV = $1.1 \mathrm{MM}$ ).

FIGURE 5.31. SHORTER HEATER STRIPS SURVIVED THE BRIDGE RELEASE PROCESS WITH POOR ADHESION AND WERE DISLODGED FROM THE FORCE OF A PROBE TIP OR WIRE BONDER. THIS BRIDGE FROM D2-8 IS 500 $M \mathrm{M}$ LONG.

FIGURE 5.32. EXAMPLE OF REDEPOSITION OF POLYMER FLAKES DURING A 60 MINUTE SACRIFICIAL PR ETCH FOR A WAVEGUIDE BRIDGE RELEASE IN THE TECHNICS PLASMA ETCHER .......................................132

FigURE 5.33. ALIGNING THE SCRIBE STRAIGHT EDGE TO $\{110\}$ PLANES FOR SCRIBE AND CLEAVING..........135

FIGURE 5.34. ALIGNED SCRIBE AND CLEAVE METHOD RESULTING IN A) A PC BRAGG GRATING CROSSSECTION, B) A D1 RIB WAVEGUIDE FACET, AND C) A D2 RIB WAVEGUIDE FACET ............................135

FIGURE 5.35. HANDLING TECHNIQUE FOR DIE DURING THE BRIDGE RELEASE PROCESS ..............................137

FIGURE 5.36. PHOTOMICROGRAPH SHOWING THE RELEASE OF A 100 AND $200 \mu \mathrm{M}$ BRIDGE AND COLLAPSE OF A 400 AND $800 \mu \mathrm{M}$ BRIDGE. THE ETCH FRONT BETWEEN OXIDE AND SILICON IS EVEN......................138

FIGURE 5.37. TOUSIMIS CRITICAL POINT DRYER PRESENT AT THE MCGILL UNIVERSITY MICROFAB. ..........140

FIGURE 5.38. CROSS-SECTION OF D1 DEVICE SHOWING PR INFILTRATION FROM THE BACKFILL PROCESS FOR A PARTIALLY RELEASED BRIDGE. AN 800 NM RIB WAVEGUIDE HAS BEEN ETCHED, LEAVING A VISIBLE DEVICE LAYER THICKNESS OF $1.7 \mu \mathrm{M}$.

FIGURE 5.39. CROSS-SECTION SHOWING COMPLETE PR INFILTRATION REPLACEMENT FOR A PARTIALLY

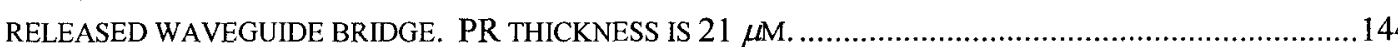

FIGURE 5.40. A) TOP DOWN PHOTOMICROGRAPH, AND B) SEM CROSS-SECTION OF THE SAME $500 \mu M$ LONG BRIDGE AFTER THE ASH RELEASE FROM ITS PR SUPPORT. STRUCTURES WERE HELD IN PLACE WITHOUT FRACTURE DURING THE CROSS-SECTION CLEAVE. ................................................................145

FIGURE 5.41. CROSS-SECTION FROM BOTH BESOI WAFERS. A) SHOWS AN "A" RELEASE PROFILE FROM THE BRIDGE TEST WAFER AND B) SHOWS A "V" RELEASE PROFILE FROM THE DEVICE WAFER.....................145

FIGURE 5.42. ISOTROPIC ETCH PROFILE OVER FOUR INTERVALS OF A HOMOGENEOUS SACRIFICIAL LAYER. 146

FIGURE 5.43. AN "A" ETCH PROFILE AS IT DEVELOPS OVER FOUR ETCH INTERVALS OF AN INHOMOGENEOUS

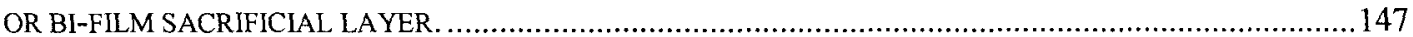

FIGURE 5.44. TRUNCATED SACRIFICIAL ETCH OF THE "V" SIDEWALL PROFILE BESOI SHOWING COMPOSITION OF WORKING FILM AND SACRIFICIAL FILM. ONCE THE FASTER ETCHING SACRIFICIAL FILM OVERTAKES THE WORKING FILM, A "V" PROFILE WOULD DEVELOP. 148 IGURE 5.45. TRUE MEANDERING SHAPE OF THE "A" SACRIFICIAL LAYER SOI SHOWN IN A) SEM CROSSSECTION AND B) TOP-DOWN PHOTOMICROGRAPH OF SAME SAMPLE. ................................................ 148

FIGURE 5.46. PHOTOMICROGRAPH OF D2-41 ("A" PROFILE SOI ) SHOWING BUCKLED $4000 \mathrm{MM}$ BRIDGE WITH OXIDE BUMPS REMAINING ON THE HANDLE LAYER (250 MM FIELD OF VIEW)..................................149

FIGURE 5.47. VARIOUS $4000 \mu$ M RELEASED BRIDGES: A) SHOWS A NEUTRAL RELEASE. B) AND C) SHOW RELEASE OF A $4000 \mu \mathrm{M}$ BRIDGE WITH SOME RESIDUAL STRESS IN THE DEVICE LAYER CAUSING SLIGHT

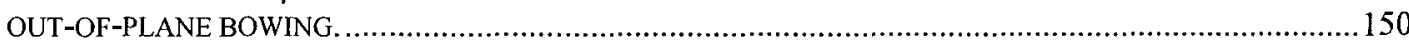

FIGURE 5.48. EXAMPLE OF MECHANICAL RUPTURE OF DEVICE LAYER SILICON ALONG AN UNEVEN ETCH FRONT DURING THE SACRIFICIAL ETCH OF "A" PROFILE SOI. ...................................................

FIGURE 5.49. UNEVEN ETCH FRONTS LEFT BY CONC. 49\% HF ON D2 BRIDGE RELEASE PROCESS

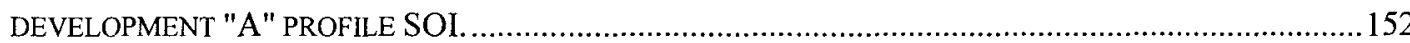

FIGURE 5.50. WEST-BOND WIRE BONDER WITH CHIP CARRIER IN PLACE ON HEATED WORKHOLDER FOR D2-

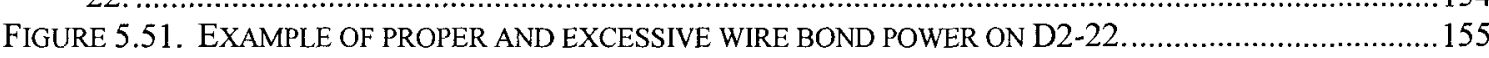

FIGURE 5.52. D2-HT1 WITH HEATERS TO TEST PACKAGING, BONDING, AND ELECTRICAL CHARACTERISTICS.

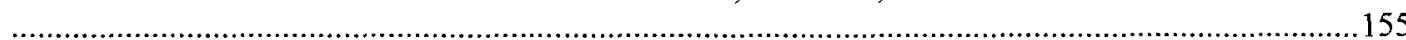

FIGURE 6.1. TEST FOR OHMIC CONTACT OF WIREBOND TO HEATER STRIPS ON D2-HT1 ........................... 159

FIGURE 6.2. SCHEMATIC FOR ELECTRICAL CHARACTERISATION OF INTEGRATED HEATERS........................159

FIGURE 6.3. PACKAGED D1-3 DEVICE UNDERGOING ELECTRICAL TESTING. THE CAPABILITY EXISTS FOR SIMULTANEOUS OPTICAL CHARACTERISATION. ... 160

FIGURE 6.4. VOLTAGE VERSUS CURRENT FOR DEVICE D1-3

160 
FiguRE 6.5. PACKAGED D2-22 DEVICE CONFIGURED FOR SIMULTANEOUS ELECTRO-THERMAL AND THERMO-

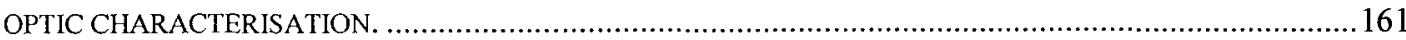

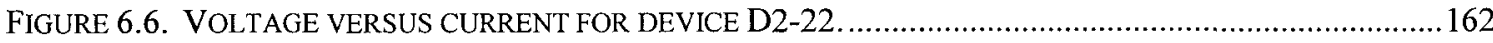

FIGURE 6.7. CALCULATED HEATER RESISTANCE VERSUS THE TEMPERATURE CALCULATED FROM THE TCR OF

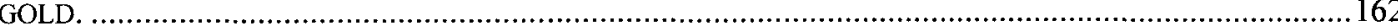

FIGURE 6.8. TEST SETUP FOR EXTERNAL HEATING FROM A LASER DIODE FOR THERMO-MECHANICAL BRIDGE

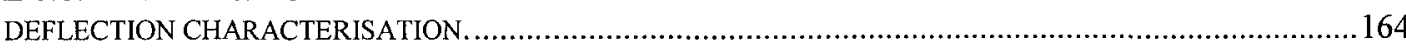

FIGURE 6.9. IMAGE OF LASER DIRECTED ONTO A SMOOTH SILICON SURFACE ON WHICH TEST BRIDGES HAVE

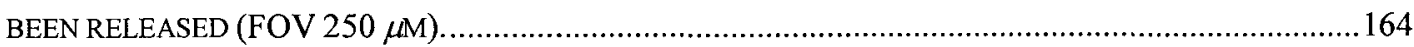

FIGURE 6.10. LD DRIVE CURRENT VERSUS LD POWER, INDICATING REVERSIBILITY IN THE LD

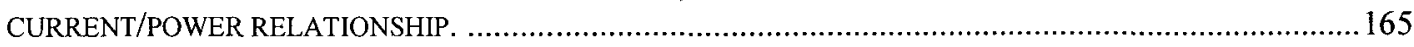

FIGURE 6.11. LD DRIVE CURRENT VERSUS LD POWER PLOTTED TO SHOW THE LINEARITY OF THE LD POWER OVER THE RANGE OF THERMO-MECHANICAL CHARACTERISATION. THE LD CURRENT AT WHICH A 2000

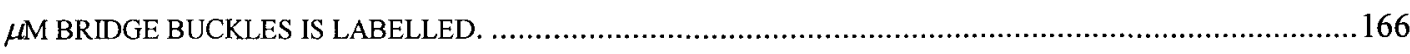

FIGURE 6.12. ALTERNATIVE METHOD OF EXTERNAL HEATING BY GUIDING THE LASER LIGHT DIRECTLY TO THE BRIDGE THROUGH A FIBRE. ALSO SHOWN IS THE BRIDGE DEFLECTION CAMERA ORIENTATION TO CAPTURE BUCKLE HEIGHT.

FIGURE 6.13. THE $123 \mu \mathrm{M}$ WIDTH OF THE LASER ACTUATION FIBRE USED TO CALIBRATE THE RULER IN PHOTOSHOP ELEMENTS FOR DEFLECTION HEIGHT MEASUREMENTS (FOV $250 \mu \mathrm{M}$ ) ......................167

FIGURE 6.14. A) MASK ALIGNMENT CROSS STRUCTURE CAPTURED BY THE BRIDGE DEFLECTION CAMERA FOR PIXEL ASPECT RATIO VERIFICATION. CROSS ON THE LEFT IS ENCLOSED BY GOLD DEPOSITION FROM HEATER MASK ALIGNMENT SQUARE, CROSS ON THE RIGHT IS BARE SILICON. B) SAMPLE LINE WIDTH MEASUREMENT ON THE SAME STRUCTURE FROM LINE WIDTH METROLOGY SYSTEM IN FABRICATION LAB.

FIGURE 6.15. PHOTOMICROGRAPHS OF LASER HEATING DEFLECTING A $2000 \mu \mathrm{M}$ LONG, $5.0 \mu \mathrm{M}$ THICK, $30 \mu \mathrm{M}$ WIDE BRIDGE INTO THE POST-BUCKLE REGIME WITHOUT AND WITH AN 800 NM LASER LIGHT FILTER $(\mathrm{FOV}=250 \mu \mathrm{M})$.

FIGURE 6.16. $500 \mu \mathrm{M}$ LONG, 890 NM DEEP RIB WAVEGUIDE BRIDGE IN POST-BUCKLE REGIME (D2-16 WG-A).

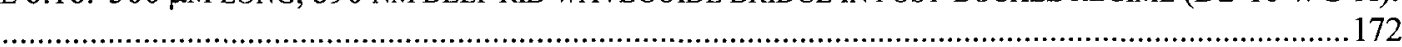

FIGURE 6.17. $2000 \mu \mathrm{M}$ LONG, 890 NM DEEP RIB WAVEGUIDE BRIDGE IN POST-BUCKLE REGIME USING THE 123

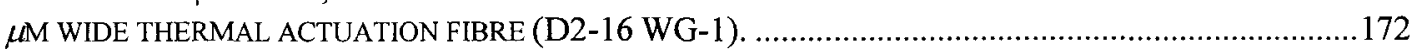

FIGURE 6.18. CRITICAL BUCKLE HEIGHT VERSUS BRIDGE LENGTH FOR A $30.9 \mu \mathrm{M}$ WIDE, $5 \mu \mathrm{M}$ THICK

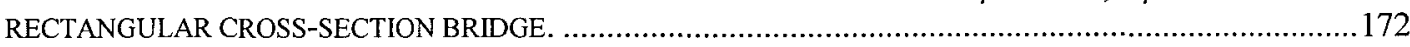

FIGURE 6.19. CRITICAL BUCKLE HEIGHT VERSUS BRIDGE LENGTH FOR A $30.7 \mu \mathrm{M}$ WIDE, 890 NM DEEP RIB

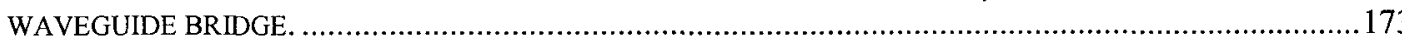

FIGURE 6.20. $2000 \mu \mathrm{M}$ LONG, 890 NM DEEP RIB WAVEGUIDE BRIDGE AT REST, $0.5 \mu \mathrm{M}$ BUMP DEFLECTION,

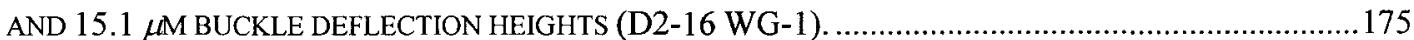

FIGURE 6.21. DEFLECTION HEIGHT VERSUS LD CURRENT FOR D2-16 WG-1 .....................................176 FIGURE 6.22. IMS-NRC OPTICAL TEST BENCH WITH THE PC-1 TEST DIE IN PLACE FOR CHARACTERISATION.

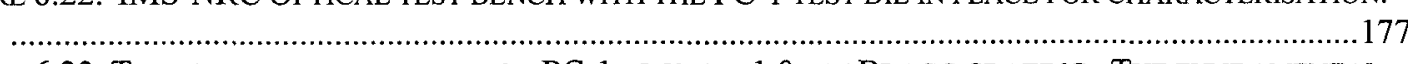

FIGURE 6.23. TRANSMISSION SPECTRUM FOR PC-1 ALONG A 1.0 MM BRAGG GRATING. THE FUNDAMENTAL MODE OF THE BRAGG WAVELENGTH AT 1555.6 NM IS SHOWN ALONG WITH THREE HIGHER ORDER LEAKY MODE WAVELENGTHS.

FIGURE 6.24. RESULTS FROM 4MM BRAGG GRATING PUBLISHED BY T. MURPHY [115] ..........................179

FIGURE 6.25. FIBRE OPTIC U-BENCH USED TO ALLOW THE INSERTION OF A 1100 NM EDGEPASS FILTER INTO

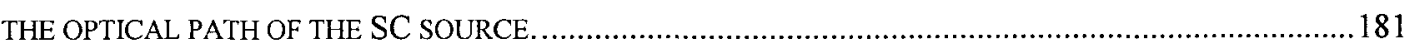

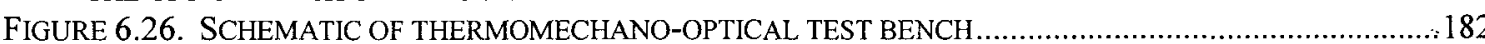

FIGURE 6.27. THERMOMECHANO-OPTICAL TEST BENCH WITH SC LIGHT SOURCE (IMAGE REVERSED)........182

FIGURE 6.28. CORE OF THERMOMECHANO-OPTICAL TEST BENCH (IMAGE REVERSED) ...............................183

FIGURE 6.29. PART OF THE FIBRE TO WAVEGUIDE ALIGNMENT PROCESS IN THREE-SPACE. .......................184

FIGURE 6.30. NEAR-IR IMAGE OF $1 \mathrm{MW} 1550$ NM LIGHT FROM OUTPUT FACET.........................................184

FIGURE 6.31. DIRECT SPECTRAL RESPONSE OF SC SOURCE TO OSA VIA OPTICAL PATCH CORD...................185

FIGURE 6.32. FIBRE-TO-FIBRE AND FIBRE-TO-STRAIGHT WAVEGUIDE ALIGNMENT. .................................186

FIGURE 6.33. TRANSMISSION RESPONSE TO DETERMINE THE AMOUNT OF LOSS FROM EACH ELEMENT OF THE TEST SETUP, TAKEN OVER 1300 - 1600 NM 
FIGURE 6.34. COMPARISON OF THE SPECTRAL RESPONSE OF A STRAIGHT WAVEGUIDE TO A BRAGG GRATING WAVEGUIDE FOR D2-16. THE BRAGG WAVELENGTH RESPONSE WAS SHIFTED 4 DB DOWN.

FIGURE 6.35. TRANSMISSION SPECTRUM OF A $1000 \mu \mathrm{M}$ BRAGG GRATING AT COURSE AND FINE BANDWIDTHS FOR D2-16 WG-1 AT ROOM TEMPERATURE. THE FUNDAMENTAL MODE BRAGG WAVELENGTH IS

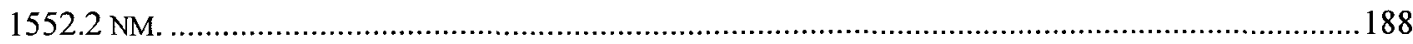
FIGURE 6.36. PLOT OF A HIGHER ORDER MODE BRAGG DIP FROM D2-16 SHOWING TWO PEAKS AND THE METHOD TO RESOLVE THE BRAGG WAVELENGTH, 1315.1 NM IN THIS CASE.

FIGURE 6.37. SEM IMAGES OF D2-24 AND D2-14 SHOWING THE RIB WAVEGUIDE / BRAGG GRATING OVERLAP THAT CAUSES TWO DIFFERENT DUTY CYCLES FOR THE SAME BRAGG GRATING PITCH.........190

FIGURE 6.38. SCHEMATIC FOR THERMO-OPTICAL CHARACTERISATION OF DEVICE WITH INTEGRATED

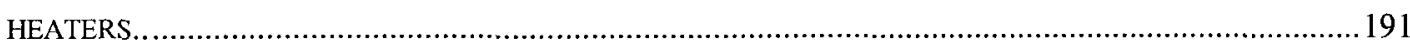

FIGURE 6.39. PACKAGED D2-22 DEVICE UNDERGOING SIMULTANEOUS ELECTRO-THERMAL AND THERMO-

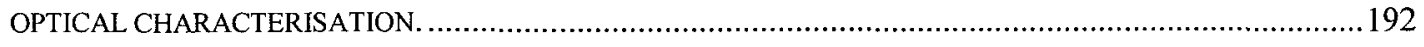

FIGURE 6.40. THE SHIFT IN BRAGG WAVELENGTH VERSUS THE TEMPERATURE FROM AN INTEGRATED HEATER OF A BRAGG GRATING FILTER WITHIN A WAVEGUIDE..........................................................193

FIGURE 6.41. COMPARISON OF THERMAL SENSITIVITY IN THE BRAGG WAVELENGTH OF HIGHER ORDER

LEAKY MODES ON D2-22
FIGURE 6.42. SHIFT IN BRAGG WAVELENGTH OF HIGHER ORDER LEAKY MODE VERSUS LASER DIODE CURRENT USING D2-16 IN THE PRE-BUCKLE REGIME. ........................................................... 196

FIGURE 6.43. WAVEGUIDE BRIDGE DEFLECTION VERSUS THE WAVEGUIDE BRIDGE TEMPERATURE OVER THE PRE-TO POST-BUCKLE REGIME FOR D2-16 WG-1.

IGURE 6.44. UNPACKAGED D2-16 DEVICE UNDERGOING THERMOMECHANO-OPTICAL CHARACTERISATION.

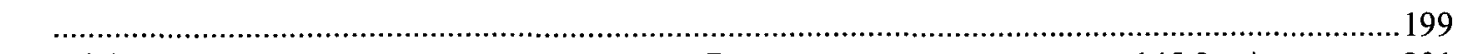

FIGURE 6.45. 20 NM RESOLUTION SCAN OF PRE-BUMP BRAGG WAVELENGTH PLOT AT 145.0 MA. ............201

FIGURE 6.46. 5 NM RESOLUTION SCAN OF PRE-BUMP BRAGG WAVELENGTH PLOT AT 145.0 MA.................201

FIGURE 6.47. 20 NM RESOLUTION SCAN OF POST-BUMP BRAGG WAVELENGTH PLOT AT $146.0 \mathrm{MA} \ldots \ldots \ldots \ldots . .202$

FIGURE 6.48. 5 NM RESOLUTION SCAN OF POST-BUMP BRAGG WAVELENGTH PLOT AT 146.0 MA..............202

FIGURE 6.49. 20 NM RESOLUTION SCAN OF PRE-BUCKLE BRAGG WAVELENGTH PLOT AT 153.0 MA...........203

FIGURE 6.50. 5 NM RESOLUTION SCAN OF PRE-BUCKLE BRAGG WAVELENGTH PLOT AT 153.0 MA.............203

FIGURE 6.51. 20 NM RESOLUTION SCAN OF POST-BUCKLE BRAGG WAVELENGTH PLOT AT 154.0 MA...........204

FIGURE 6.52. 5 NM RESOLUTION SCAN OF POST-BUCKLE BRAGG WAVELENGTH AT 154.0 MA. ................204

FIGURE 6.53. PLOT OF THE BRAGG FILTER WAVELENGTHS BEFORE AND AFTER BUMP DEFLECTION AND BEFORE AND AFTER BUCKLE DEFLECTION OF THE WAVEGUIDE BRIDGE FILTER. .............................20

FIGURE 6.54. THERMOMECHANO-OPTICAL RESPONSE OF THE D2-16 WG-1 SUSPENDED WAVEGUIDE FILTER.

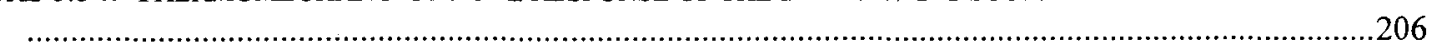

FIGURE 6.55. THEORETICAL THERMOMECHANO-OPTICAL RESPONSE OF DEVICE AS A RESULT OF ANALYSIS OVER THE PRE-BUCKLE, BUMP, BUCKLE, AND POST-BUCKLE REGIMES........................................208

FIGURE 6.56. OVERLAY OF THERMOMECHANO-OPTICAL AND THERMO-MECHANICAL RESPONSE OF D2-16 WG- 1

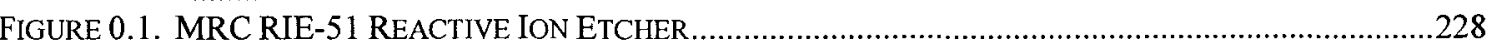

FIGURE 0.2. RIE RESULTS IN WHICH PART OF THE BOX HAS BEEN ETCHED. THE PHOTORESIST DEFINING THE BRIDGE WIDTH HAS NOT YET BEEN REMOVED IN THESE SEM CROSS-SECTIONS .............................229

FIGURE 0.3. BOTH DIE HAVE GONE THROUGH THE HF BRIDGE ETCH. THE PR ON THE LEFT SAMPLE WAS PROCESSED WITH AN HMDS SPIN COAT. THE PR ON THE RIGHT SAMPLE WAS PROCESSED IN AN HMDS

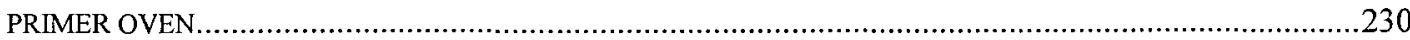

FIGURE 0.4. CHROMIUM ADHESION ON THE LEFT (D2-30) AND TITANIUM ADHESION ON THE RIGHT (D2-19) AFTER THE PR PASSIVATION LAYER WAS REMOVED VIA O ${ }_{2}$ PLASMA ASHING. .................................230

FIGURE 0.5. D2-19 ON THE LEFT SHOWS WHERE THE PHOTORESIST PASSIVATION HAS FAILED AFTER THE HF BRIDGE RELEASE. D2-8 ON THE RIGHT SHOWS AREAS OF FAILURE ALONG THE STEP HEIGHT DIFFERENTIAL AFTER THE HF BRIDGE RELEASE ETCH AND PR PASSIVATION REMOVAL....................231

FIGURE 0.6. EXAMPLE OF STEP HEIGHT COVERAGE PROBLEM WITH D2-30 ALONG WITH HF RELEASE........232

FIGURE 0.7. D2-8 HEATERS ON THE 500 MICRON BRIDGE SURVIVED THE HF BRIDGE RELEASE PROCESS THOUGH THE HEATERS COULD NOT BE SUCCESSFULLY ADDRESSED WITH EITHER PROBE TIPS OR WIRE BONDING. 


\section{Introduction}

Producing three dimensional integrated devices in the field of microsystem technology (MST) draws upon a wide variety of sciences that interact with the physical world. These devices are either the microscopic equivalent of a macroscopic structure $[1,2,3]$, or the enabling component of a larger system $[4,5,6]$. The processes used to fabricate these devices are generally called micromachining or nanomachining. Depending on the scale, the devices themselves are known as microelectromechanical systems (MEMS) or nanoelectromechanical system (NEMS). MEMS refer to device structures that measure on a scale in the millimetre to micron range, while NEMS describes structures scaled to the nanometre range. When a microsystem incorporates optical elements into its functionality, the device is referred to as a Microoptoelectromechanical system (MOEMS).

Integrated optics and micro-optics make use of many of the same fabrication processes that MEOMS based devices do. They can be considered to be "solid-state" in the sense that there are no moving or mechanical elements to the design. This class of devices can include such microstructured geometries as Bragg gratings, photonic crystals, ring resonators, arrayed waveguide gratings (AWG) or Mach-Zehnder interferometers (MZI).

When choosing a micro-optics approach the engineer must consider such factors as desired range of performance and relative simplicity of process flow in the design process. It is also possible to take a proven micro-optic device and enhance its performance by introducing MOEMS device elements with functionality such as environmental isolation [7] or greater tunability such as the mechanically actuated anti- 
reflection switch (MARS) [8].

The bulk of MOEMS based devices can be arranged into the following categories: sensors and actuators, optical scanners, communications, display and imaging, and adaptive optics [9]. In addition to these specific device categories, there can also be considerable "cross-pollination" between them. For example, the tilting mirror design responsible for the functionality of the Lucent Lambda Router all-optical cross-connect communications device is essentially the same enabling element as the seminal TI DMD (Texas Instruments Digital Micromirror Device) display and imaging system.

This section has thus far dealt mainly with the structural element of MOEMS devices. Materials are just as important to the design process and device performance. In the field of MEMS and MOEMS, silicon, particularly single crystal silicon, is an ideal material for device fabrication [10]. Included in its many advantages are its mechanical properties. Single crystal silicon has a similar stress to strain ratio, also known as modulus of elasticity, as steel. It is also only elastically deformed at low temperature and thus has no mechanical hysteresis in this regime. This is demonstrated in figure 1.1 where a deflected silicon cantilever will return to its original shape when the probe tip is removed [11]. This is an important property when considering the stability of a device whose mechanical component will be cycled over a regular interval.

There are a variety of fabrication process options available, due to silicon's anisotropic wet etch characteristics, in addition to the more complicated dry etch processes. Figure 1.2 demonstrates that by choosing the proper wafer and mask alignment, an optical rib waveguide can be defined by anisotropically wet etching a trench on either side of the rib [12]. With proper mask alignment the sidewall of the 
waveguide will be nearly, in theory, atomically smooth.

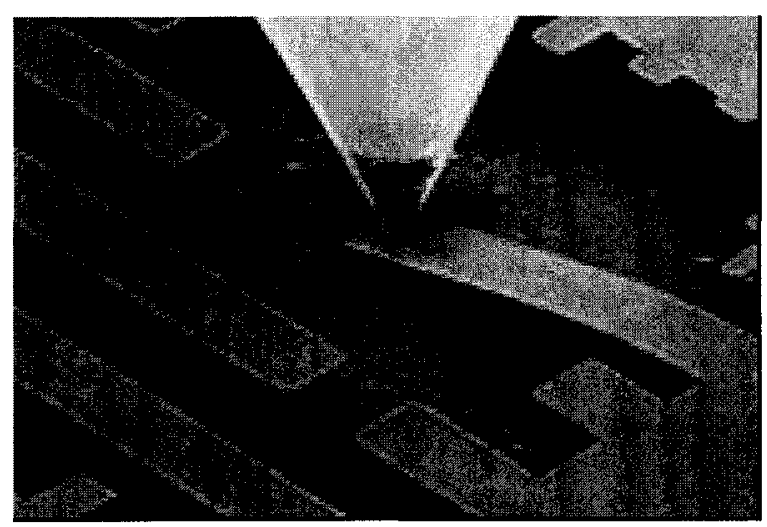

Figure 1.1. Deflection of silicon micromachined cantilever to demonstrate its mechanical properties [11].

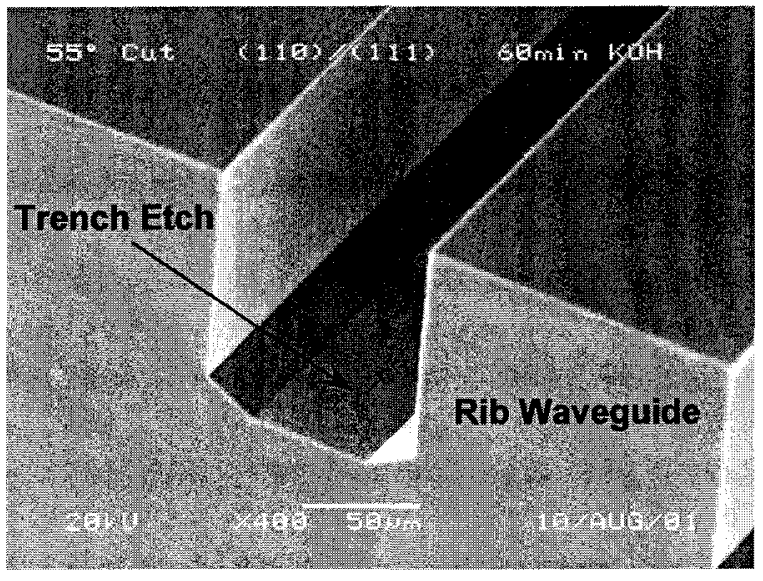

Figure 1.2. Anisotropic wet etch of $(110) /(111)$ silicon at $55^{\circ}$ from the (100) flat. The trenches define a rib waveguide [12].

As a semiconductor, silicon is also a natural match when the electronic properties of the material or the integration of electronic components are important to the design. Well established process flows also allow for the integration of such electrical components as resistors and capacitors into MEMS and MOEMS devices made of silicon. These elements can be added monolithically to the silicon platform as shown in figure 1.3. One example is the previously mentioned TI DMD. Each micro-mirror pixel is monolithically integrated on top of the electronic CMOS bit that controls that mirror 
[13]. Another example is the family of Analog Devices accelerometers. These devices are fabricated using both their iMEMS and SOI-MEMS processes [14]. Within the sensor, the micromachined proof-mass structure is integrated with the signal process and control circuitry on a single silicon substrate. The MEMS component of each device is realized through the surface micromaching fabrication process.
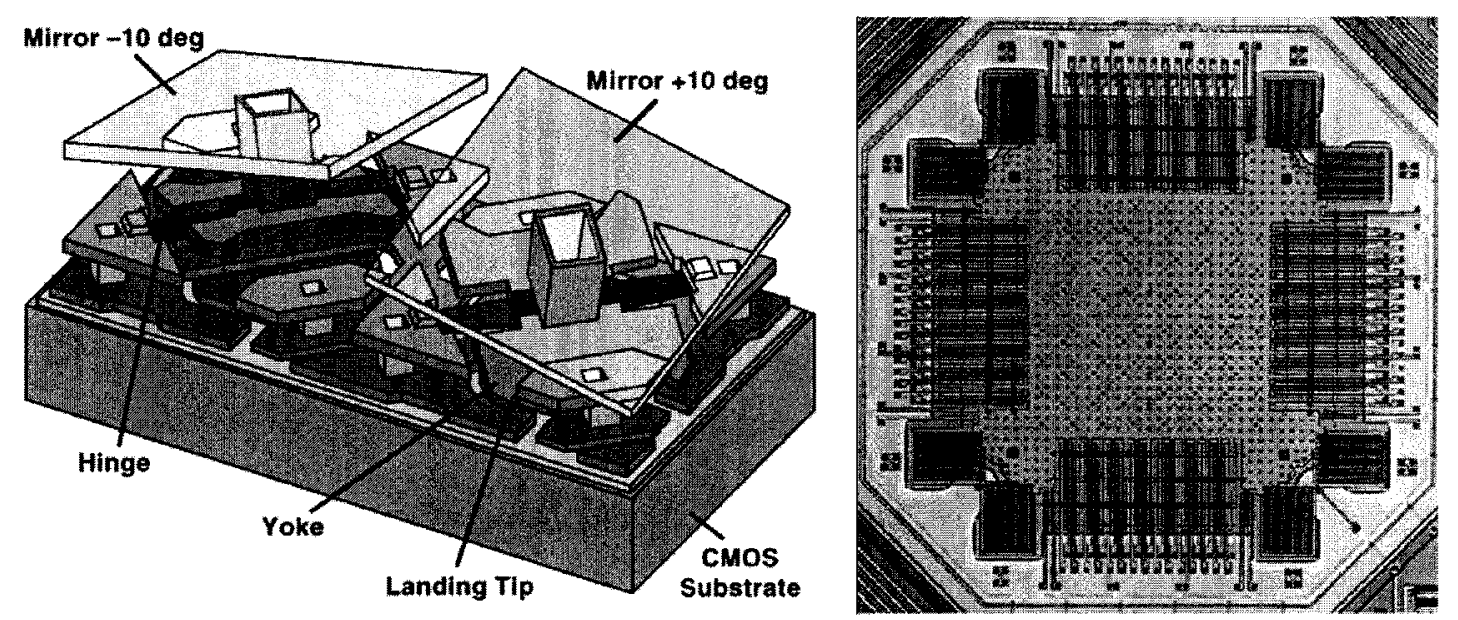

Figure 1.3. The TI DMD and Analog Devices Accelerometer [13,14]

The optical properties of silicon are such that the absorption band edge of silicon occurs at a wavelength of $1.1 \mu \mathrm{m}$ as shown in figure 1.4 in comparison to other direct and indirect band gap materials [15]. The absorption of shorter wavelengths makes silicon useful for photodetectors, while its transmission in the near-IR wavelengths makes silicon suitable for photonic devices in the optical communication bands. The high thermo-optic coefficient of silicon also makes it an excellent material candidate for thermally tuned MOEMS and micro-optic devices.

Since silicon will be the substrate for integration of the MEMS, micro-optic, and electrical elements of the device presented in this thesis; the sacrificial material to release the microstructures will be silicon dioxide. Silicon-on-insulator (SOI) fits this profile and is a convenient platform that will be ideal for development of the device. 


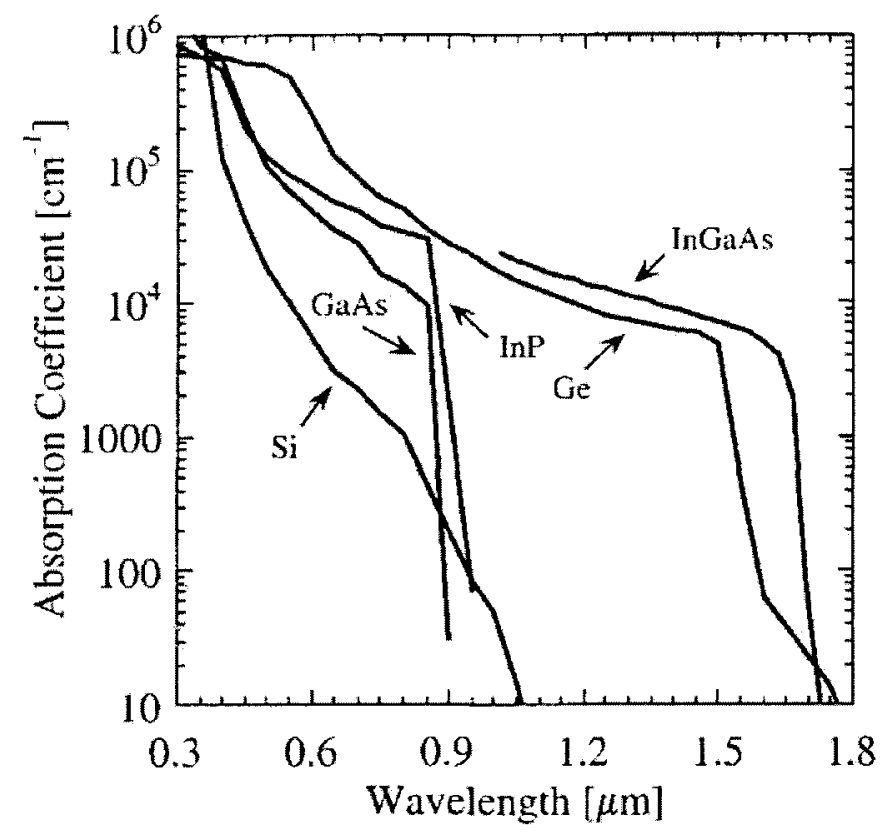

Figure 1.4. Absorption coefficient for silicon [15].

SOI was introduced to improve isolation of the $n$ and $p$ doped structures in an integrated circuit, as well as to help isolate the circuit from the bulk of the silicon to improve power consumption. Composed of three material layers, as shown in figure 1.5, the top film is called the device layer. It is generally single crystal silicon with some degree of $p$ or $n$ doping. Under the device layer is the buried oxide (BOX) layer. This is generally amorphous silicon dioxide and its formation depends on how the SOI is produced. The final layer, called the handle layer, is a standard silicon wafer. It accounts for the bulk of the SOI material and has a thickness similar to a bare silicon wafer of the same diameter.

There are numerous ways to prepare SOI for commercial use. The performance characteristics of the device to be fabricated will determine the composition of the device layer and BOX layer, as well as their respective thicknesses. One technique of SOI preparation is a method whereby oxygen is implanted into the silicon and then diffused to 
a depth that will define the thickness of the device layer. This is called SIMOX (Separation by Ion Implantation of Oxygen) SOI. Another method involves the use of two silicon wafers. A layer of thermal oxide is grown on either or both silicon wafers and the wafers are fused together. The wafer that will ultimately form the device layer is then chemo-mechanically polished (CMP) back to the desired device layer thickness. This is called BESOI (Bonded and Etched-back SOI). An SOI fabrication technique that also makes use of thermal oxide for the BOX layer is the "Smart Cut" process. Instead of CMPing the device wafer to its desired thickness from the original wafer, hydrogen is implanted to a depth that forms a plane of micro cavities. This plane separates under a heat treatment, removing the bulk of the material with only a minor CMP to finish the surface. The manufacturer of this type of SOI, SOITEC, claims greater device layer uniformity than current BESOI wafers. This uniformity is an important factor to consider should the device proceed to the manufacturing process development stage.

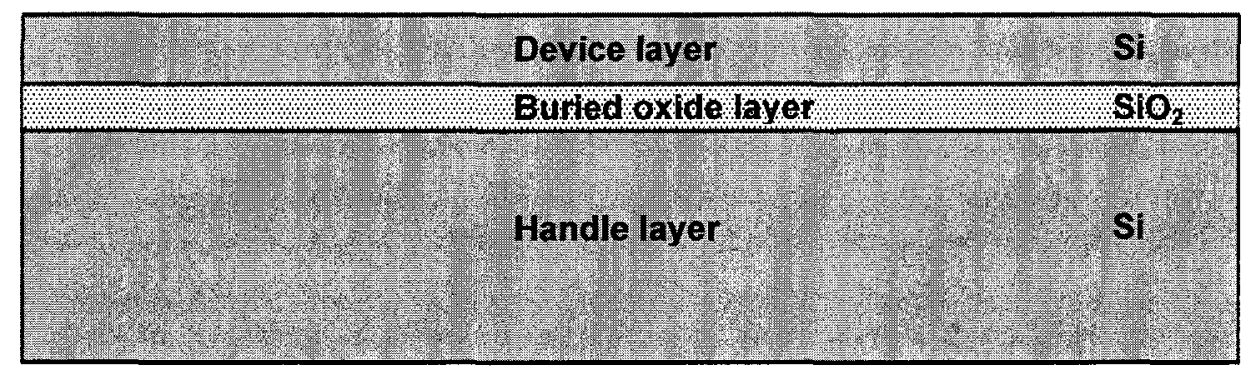

Figure 1.5. Cross-section of silicon-on-insulator (SOI) wafer.

Using the implantation method, there is a limited depth the oxygen can penetrate in the silicon to form the BOX. The BOX thickness itself will also be limited. The fusion method can produce a device layer thickness limited only by the source wafer itself. The BOX can be grown to a much greater thickness as well. The desired SOI characteristics will depend on the class of device to be fabricated. MEMS devices that 
are easier to process on thicker device and BOX layers might conflict with thinner films that are ideal for the electronic or optical components of a device. As is often the case, a successful device will depend on whether the design rules can be reconciled within the tolerance of each component of the device.

Fabrication of a MOEMS on an SOI platform provides much potential to develop a device that combines material properties and structural design in interesting and novel ways. MEMS/MOEMS is often considered to be the enabling element within a system and not a device unto itself. To explore how material and structure can be synergistically combined, an enabling component must be considered.

In the context of optical devices, the enabling component can include such elements as a filter, grating, lens, mirror, or waveguide, to name a few. The use of material properties to control these elements include thermo-optic, electro-optic, or stress-optic effects; and in more exotic devices, magneto-optic effects. The mechanical means of controlling the optical behaviour of the component can include the use of electro-static, thermal, shape memory alloy, magnetic and piezo-electric actuation on such structures as cantilevers, bridges, membranes, suspended plates, and interdigitated comb structures.

When using these various material and structural elements in the design of a MEMS/MOEMS device, the philosophy is often to use the MEMS actuator to separately manipulate the material of the enabling optical element. This design strategy can have two drawbacks. The first involves the use of space on the device substrate. For example, in the case of an electrostatic comb actuator that moves a guillotine shutter to switch light [16], much space could be saved if the mirror and actuator were physically the same 
structure. The same problem is seen with an electrostatic comb actuator that is used to buckle a fixed-fixed bridge structure [17]. These devices are shown in figure 1.6. If the bridge were to be its own actuator, the design would be much more compact and space would be saved on the substrate.
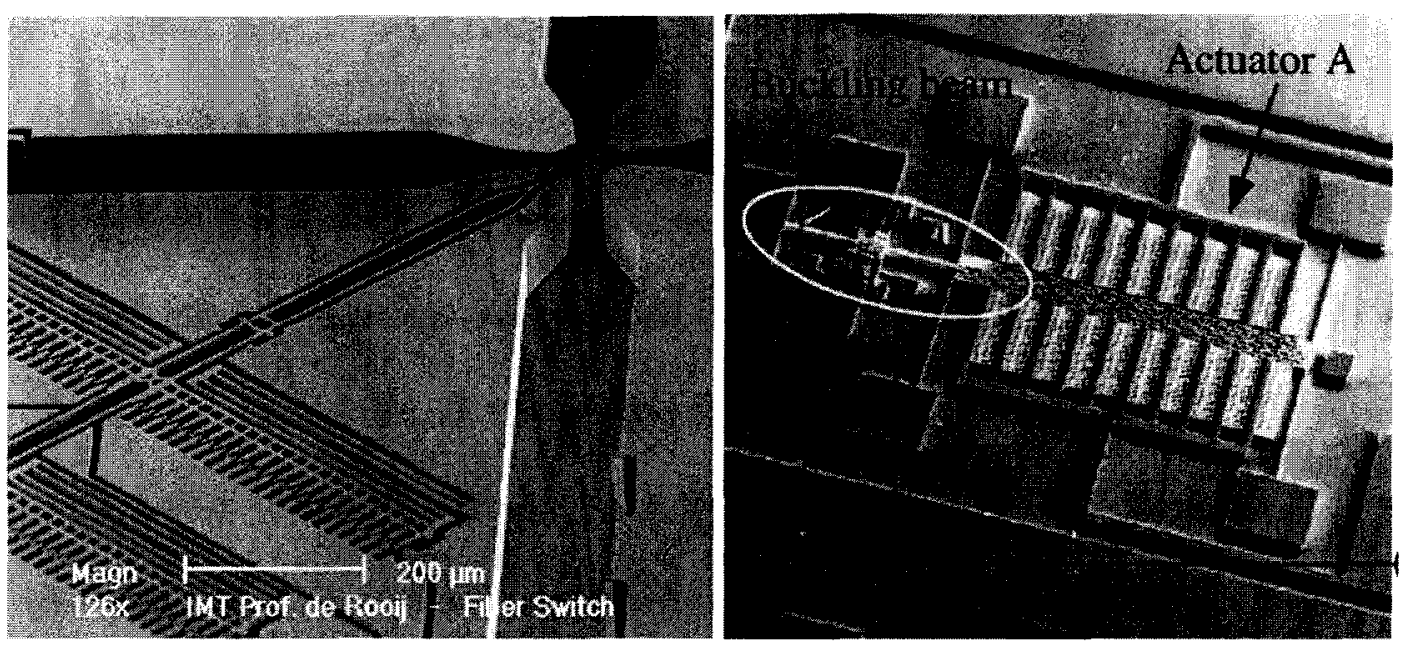

Figure 1.6. Examples of large actuator structures used to tune small enabling components $[16,17]$.

An equally important concept when considering design philosophy is the potential for performance enhancement if the material property effects of the optical element can be coupled to the tuning action of the actuator. This potential can be wasted if the actuator is used to manipulate the optical element separately. One example is the case of actuating a micro-mirror for scanning or switching light by attaching it to an electrothermally buckled bridge $[18,19]$. While this design strategy deals with the real estate issue by thermally actuating the bridge, the optical device is still separately attached to the actuator. An example of this design concept is shown in figure 1.7.

The key to reducing the use of space on the die, and taking advantage of potential coupled material effects to enhance the performance of the device, is to incorporate the mechanical and optical functionality into a single structure. One example is an electro- 
static comb actuator which is itself the waveguide for a 1 X 2 switch [20]. Another example is an electro-statically actuated cantilever in which the cantilever itself is the top half of a distributed Bragg reflector (DBR) [21].

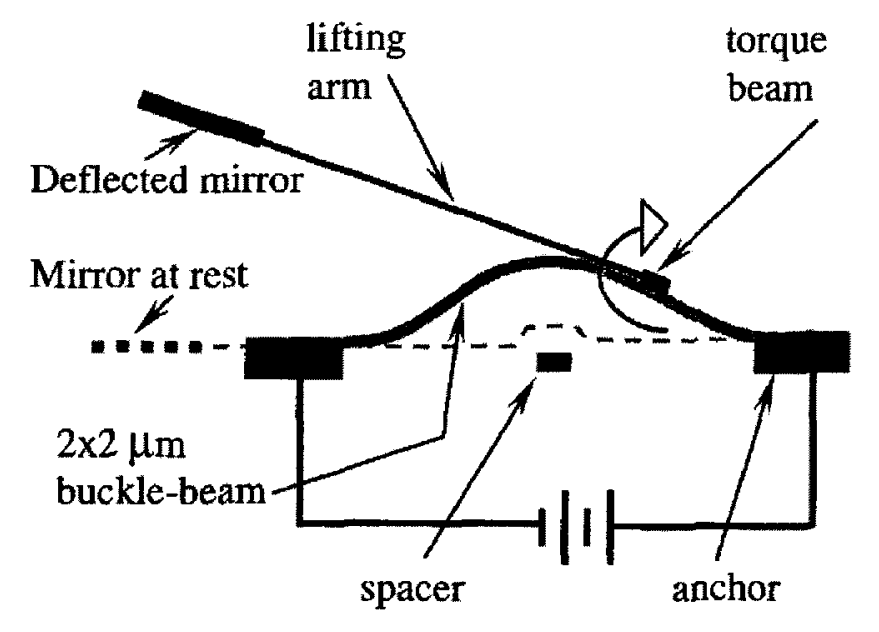

Figure 1.7. Buckle beam thermal actuator to deflect mirror [18].
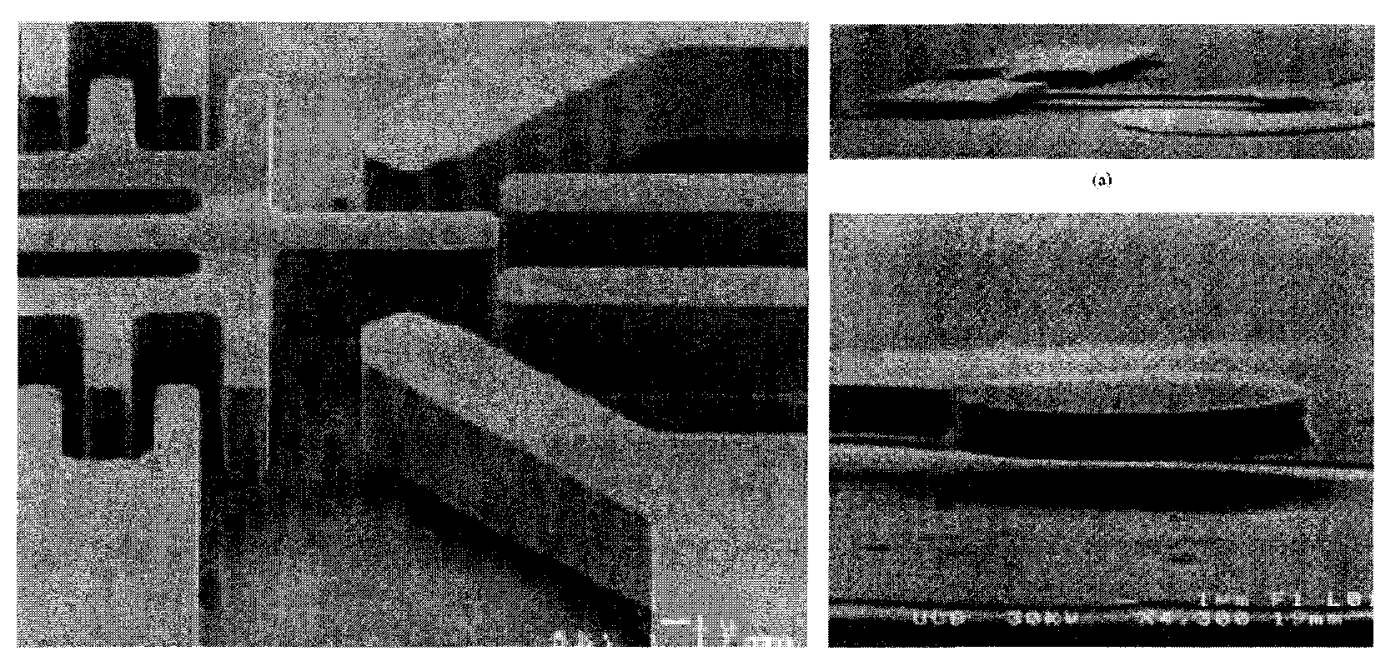

Figure 1.8. Examples of MOEMS in which the actuator is the optical component [20,21].

\subsection{Objectives}

This thesis will explore what happens when an optical device, and the actuator used to tune its response, are physically the same structure. A Bragg filter will be used to 
investigate the anticipated benefits of coupling the material and structural effects of the actuator to the optical behaviour of the device. A tunable optical filter is an important optical element that will be straightforward to characterise. The actuating structure that the optical device is integrated into will be a bridge. A bridge actuator lends itself naturally to waveguide integration so that light can be guided to the filter. The method of structural actuation will be thermal. Of the previously listed mechanical means to tune the behaviour of an optical element, thermal actuation will allow both integrated and external control. This flexibility is an important factor when developing a novel device that will bring together many elements in the design, fabrication, and characterisation process with as yet to be determined consequences.

Specific objectives have been defined to organize the investigation of a thermally tunable filter in which the actuator that tunes the filter is the filter itself. They are: 1) to develop a sacrificial release process for a fixed-fixed waveguide bridge with a high length to thickness aspect ratio; 2) to develop a prototype packaging technique that will allow for confidence and repeatability in the characterisation process; 3 ) to measure a rate of tunability before the bridge buckles that matches literature for similar non-MEMS thermo-optically tuned devices to establish a baseline optical performance; 4) to characterise the mechanical behaviour of the bridge waveguide filter as it deflects out-ofplane along with the corresponding optical response; 5) to confirm that when the bridge buckles, the wavelength of the Bragg grating filter will discontinuously shift by an amount that is in agreement with theory and; 6) to determine the difference, if any, of the Bragg filter wavelength sensitivity in $\mathrm{pm} /{ }^{\circ} \mathrm{C}$ before and after the bridge buckles.

The device will have three separate operating regimes that will be studied in order 
to characterise the result of coupling the thermal, mechanical and optical effects on the shift in the Bragg filter wavelength. In the first regime, the pre-buckle regime, the waveguide bridge will be heated, but not actuated out-of-plane. As the temperature increases, the effective index of the Bragg grating will change as a result of 1) the change in temperature, and 2) the stress resulting from constrained thermal expansion. The shift in the Bragg wavelength is sensitive to temperature and stress. Since the stress results from a temperature change, the total pre-buckle sensitivity of the Bragg wavelength can be expressed as a thermal sensitivity in $\mathrm{pm} /{ }^{\circ} \mathrm{C}$. The other element contributing to the Bragg wavelength, the grating pitch, will not change in this regime because there is no axial strain.

Figure 1.9 shows a diagram of the device in the pre-buckle configuration. The device as it is shown consists of a rib waveguide in SOI. The waveguide has been facetted so that light from input and output fibres (not shown) can be coupled into the waveguide. The centre region of the rib is patterned with the Bragg grating filter. Heaters on either side of the waveguide rib provide thermal tuning and are powered through wires at each end. The sacrificial buried oxide of the SOI is exposed through the bridge etch and wet-etched away to create the suspended rib waveguide with integrated filter.

In the second regime, the buckle regime, the bridge deflects through a transient condition to a stable buckling mode shape. As the bridge expands to this shape, the stress is released, removing the influence of the stress-optic effect. This buckle expansion also results in strain on the Bragg grating pitch, rendering the Bragg wavelength sensitive to changes in both the stress and strain within the rib waveguide filter. The buckle regime 
occurs at a characteristic temperature that results from a combination of the bridge material and geometry. Figure 1.10 depicts a cross-section of the suspended rib waveguide filter, deflected to its buckling mode shape, showing the optical mode profile confined within the rib waveguide [22]. The vertical deflection has been exaggerated to show its shape. A realistic view of the deflection height to bridge length ratio is shown in figure 6.17 of chapter 6 .

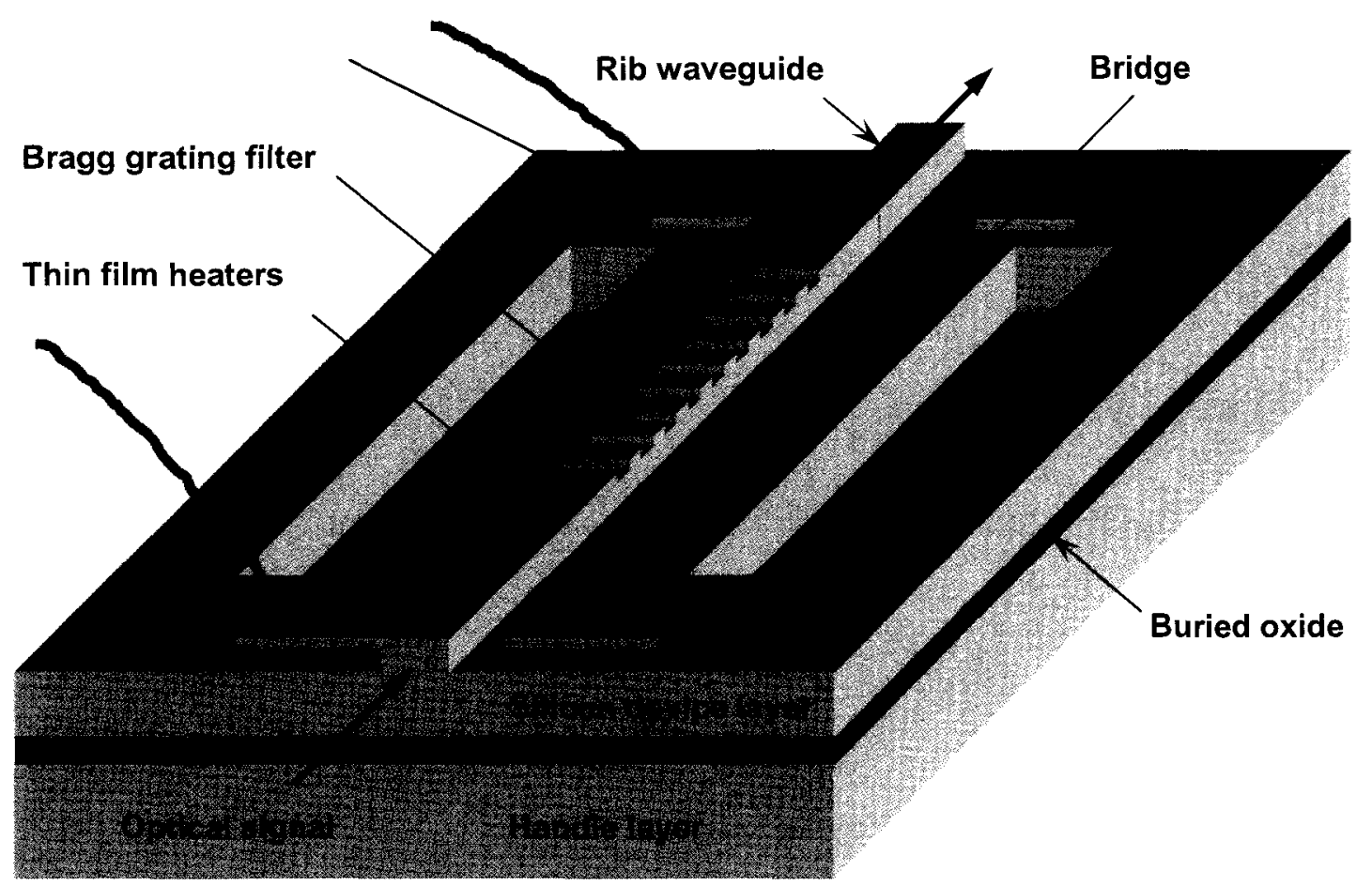

Figure 1.9. Bragg grating filter on SOI rib waveguide in pre-buckle configuration.

In the third regime, the post-buckle regime, the bridge continues to deflect steadily out-of-plane as the temperature of the bridge waveguide filter continues to increase. The effective index of the Bragg grating will once again be sensitive to temperature change. Since thermal expansion is no longer constrained, the Bragg pitch will continue to expand with a thermal sensitivity to strain. The total sensitivity of the 
Bragg wavelength in this regime is again expressed as $\mathrm{pm} /{ }^{\circ} \mathrm{C}$.

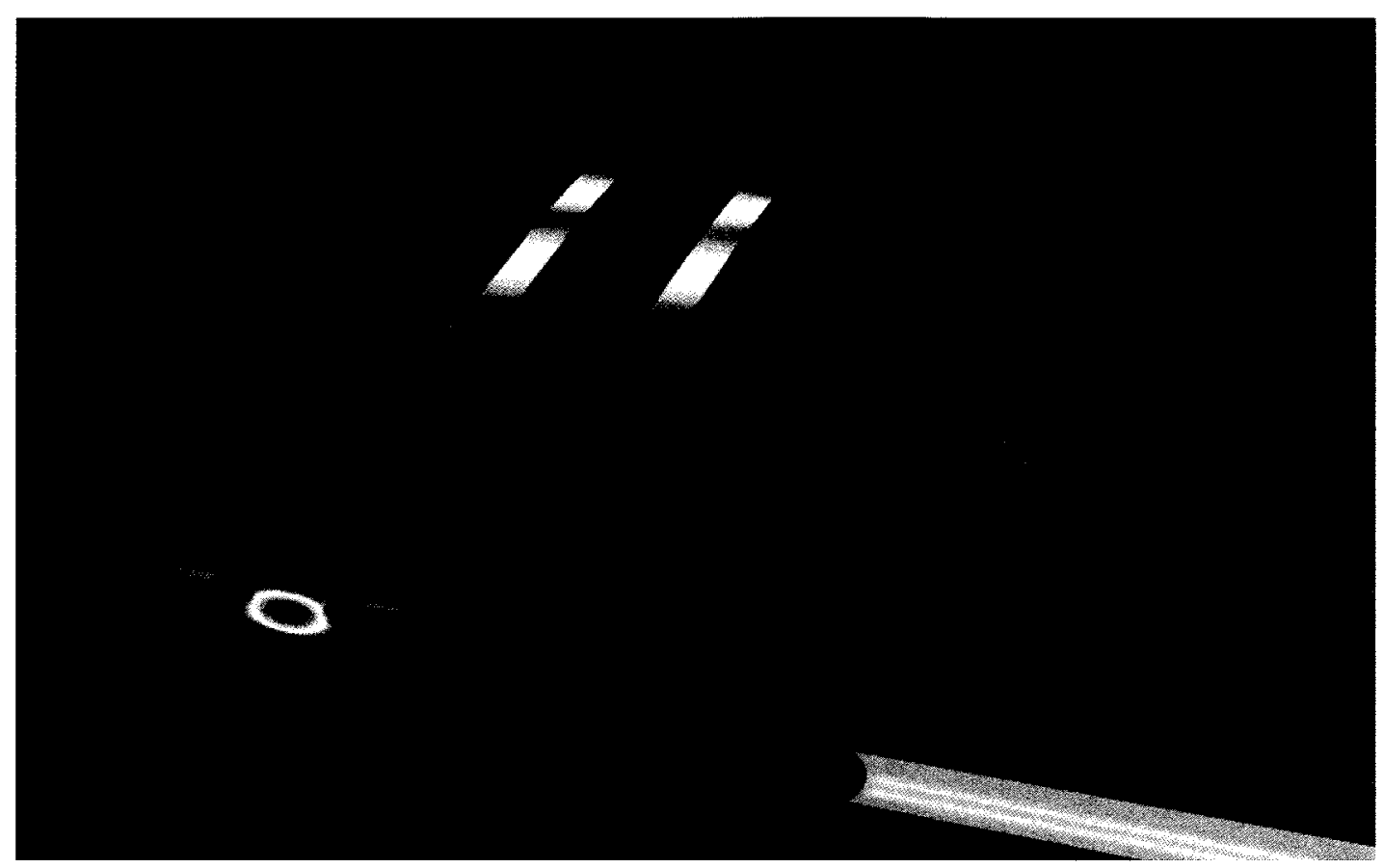

Figure 1.10. Cross-section of Bragg grating filter on SOI rib waveguide bridge in buckle mode configuration. The vertical deflection has been exaggerated to show its shape [22].

With the large number of design, fabrication, and characterisation elements at play in this thesis, the research will be an exercise in verifying the theorised behaviour from the experimental results, and not optimising those results. Chapter 5 will outline the process flow that was developed to fabricate the device shown in figure 1.9 and figure 1.10 .

\subsection{Thesis Organisation}

This thesis will use the principles of photonics, optical waves in crystals, and beam mechanics, in the context of thermal excitation, to describe a thermo-mechanically tunable suspended waveguide filter on SOI; with a focus on analytic modelling, fabrication and characterisation. Following the introduction in chapter 1, chapter 2 will 
describe the operational space the device will function in, including a survey of devices in its class, along with characteristic performance. Chapter 3 will define the theoretical elements of the device such that its performance can be reliably predicted given a set of material properties, design parameters and boundary conditions. These elements will include optical, electrical, thermal, and mechanical theory. The chapter will also begin the process of identifying ideal materials supplemental to the silicon, and geometries for the device; a process that will be finalised in the subsequent chapter. Chapter 4 collects the theory detailed in chapter 3 to generate an analytic model of the device performance and develop a design for the device. Chapter 5 documents the process flow developed to fabricate the device. Chapter 6 describes the test apparatus and experimental procedures developed to characterize the electrical, thermal, mechanical and optical performance of the device. Chapter 7 contains a discussion of the results, future work and applications. 


\section{Review of Tunable Waveguide Filters}

This survey will review research relating to waveguide filters and the spectral response that can be modified by a mechanical alteration of the Bragg grating or a physical alteration of the waveguide index itself. Devices designed from a tunable filter perform such functions as add/drop filters, dispersion compensators, noise filters, gain flatteners, sensors, spectroscopic devices and distributed-feedback lasers.

Six of the most common methods to tune the spectral response of an optical filter will be examined in this chapter. These include filters tuned by 1) piezoelectric; 2) electrostatic; 3) electromagnetic; 4) shape memory alloy (SMA) and bi-metal; 5) thermo-optic; and 6) thermo-mechanic techniques. The first four effects are mechanical in nature. Elastic stress can cause both mechanical and optical alteration in the device to achieve its tuning effect, depending on the method of actuation. The fifth effect involves optical tuning via the thermal properties of the material the device is made from. The sixth method of tuning results in a photo-elastic influence on the spectral response of the device similar to the first four, via thermal actuation. Since the device in this thesis is integrated within a thermal actuator, it will simultaneously make use of the fifth and sixth tuning methods.

\subsection{Piezoelectric Tuning}

The piezoelectric effect occurs in crystals where mechanical strain in the material produces an electric field. The change in volume (or length) caused by the strain disturbs the symmetry of the crystal and the resulting redistribution of positive and negative charges within results in a voltage being developed across the bulk of the material. 
Conversely, an applied electric field produces a mechanical strain in the structural lattice of the crystal, which in turn changes the dimensions of the crystal and produces actuation. This form of actuation applies a fast and precise displacement to the structure that the crystal has been deposited onto.

Piezoelectric tuning of optical devices has been attempted on fibre Bragg gratings (FBG) $[23,24]$ by A. Iocco, with a design detailed in figure 2.1 . The device allows both traction and compression in a fibre and can be used in both transmission and reflection. The design combines the actuated change in the grating period of the FBG, $\Delta \Lambda$, with the photo-elastic effect caused by the strain of a compressed fibre, $\Delta n$. This combination of effects shifts the Bragg wavelength peak, $\Delta \lambda_{B}$. The device was able to achieve a linear tuning range of $45 \mathrm{~nm}$ with a maximum tuning speed of $21 \mathrm{~nm} / \mathrm{ms}$.

Another more conventional use of piezoelectric based optical design is an acousto-optic device called a surface acoustic wave (SAW) filter. By using the vibration of a piezoelectric element to set up a wave of alternating densities of compression and rarefaction, a Bragg grating is created in the material. This is due to the fact that from the point of view of the propagating light wave, the SAW appears stationary $[25,26]$.

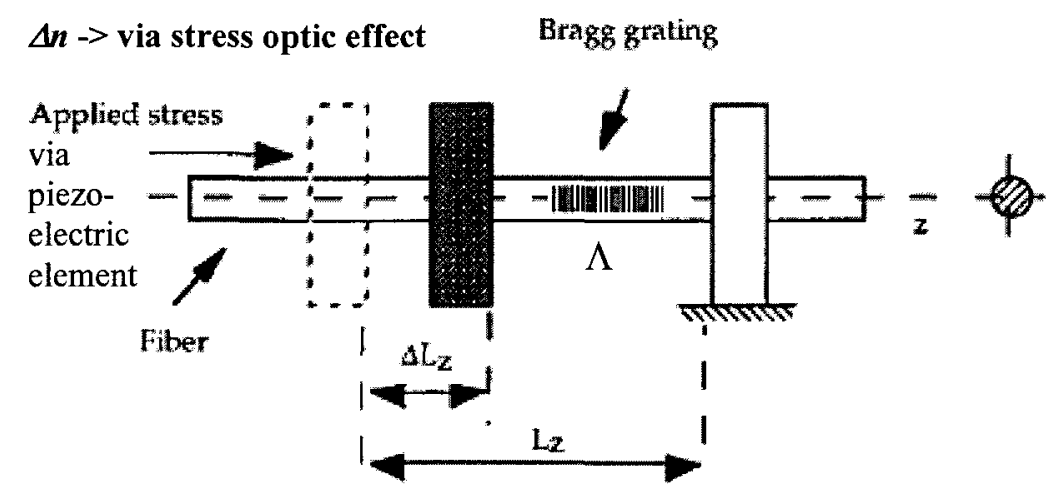

Figure 2.1: Piezoelectric axial compression of fibre Bragg grating [23]. 


\subsection{Electrostatic Tuning}

Electrostatic actuation occurs as a result of the interaction of two charged plates. When oppositely charged, the attraction is a nonlinear force-to-voltage relationship that includes the area of the plates, the voltage, and the plate separation [27]. Electrostatic actuation is a mature technology in the realm of MEMS. A variety of structures and design techniques accomplish a relatively large range of motion, in the order of microns, over a high frequency of actuation. The technique of electrostatic tuning has been applied to a variety of optical components such as filters, grating light valves, tunable cavities, and vertical-cavity surface-emitting lasers (VCSEL) [21,28,29,30].

A widely tunable torsional optical filter with a range of $100 \mathrm{~nm}$, over $1500 \mathrm{~nm}$ to $1600 \mathrm{~nm}$, with a bandwidth of $1 \mathrm{~nm}$, was developed by Carlos Mateus et al. [31]. This "see-saw" design uses one side of the cantilever as a counterweight and the other side to house two distributed Bragg reflectors (DBRs). As the pull-in force causes the counterweight to shift position, there is a corresponding change in the gap between the DBRs, changing the Bragg wavelength of reflection.

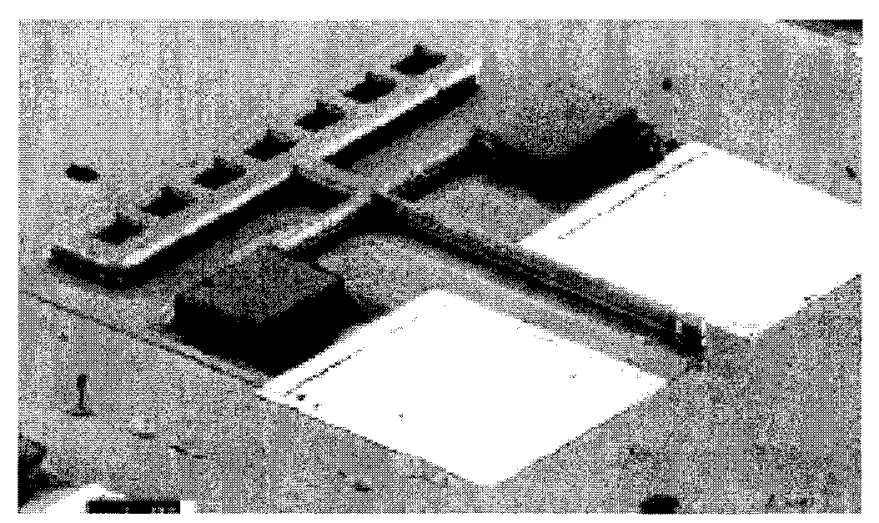

Figure 2.2. MEMS structure - DBR housed in a torsional arm cantilever [31]

An over-and-under suspended bridge design of a Fabry - Perot cavity was used 
by LeDantec et al. in InP to achieve a bandwidth of $0.6 \mathrm{~nm}$ and a tuning range of $62 \mathrm{~nm}$ [32]. The bandwidth was kept below $1 \mathrm{~nm}$ over a $40 \mathrm{~nm}$ tuning range. Essentially, this design is an elongated double pinned variation of the "suspended plate" Mechanical AntiReflection Switch (MARS) design invented by K. Goossen.

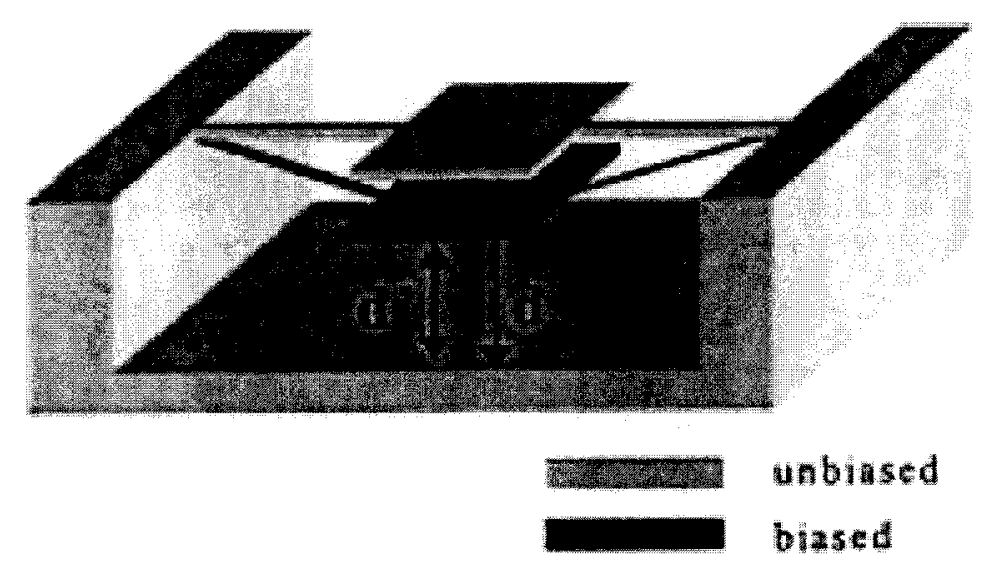

Figure 2.3: Over-and-under bridge forms Fabry - Perot cavity [32].

Tilting micro-mirrors is another design strategy that makes use of electrostatic tuning. J. Ford et al. developed a $40 \mathrm{Gbit} / \mathrm{s}$ DWDM transmission system with OADM, capable of switching $64 \times 40 \mathrm{Gbit} / \mathrm{s}$ wavelengths using a switching fabric of over 1000 mirrors [33]. The Lucent Lambda Router is an example of a commercially developed high capacity optical cross-connect (OXC) whose core enabling technology is electrostatically actuated micro-mirrors [34]. Their approach physically resembles the communications switching fabric of an electronic digital cross-connect, in which electricity is replaced with light. 


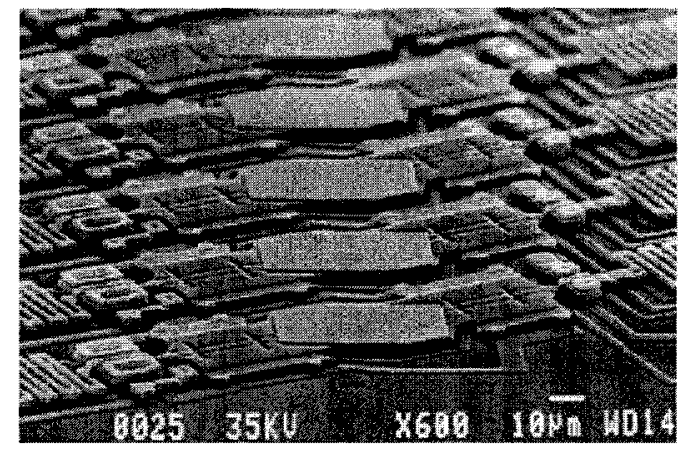

Figure 2.4: Wavelength add-drop switching using tilting micro-mirrors [33].
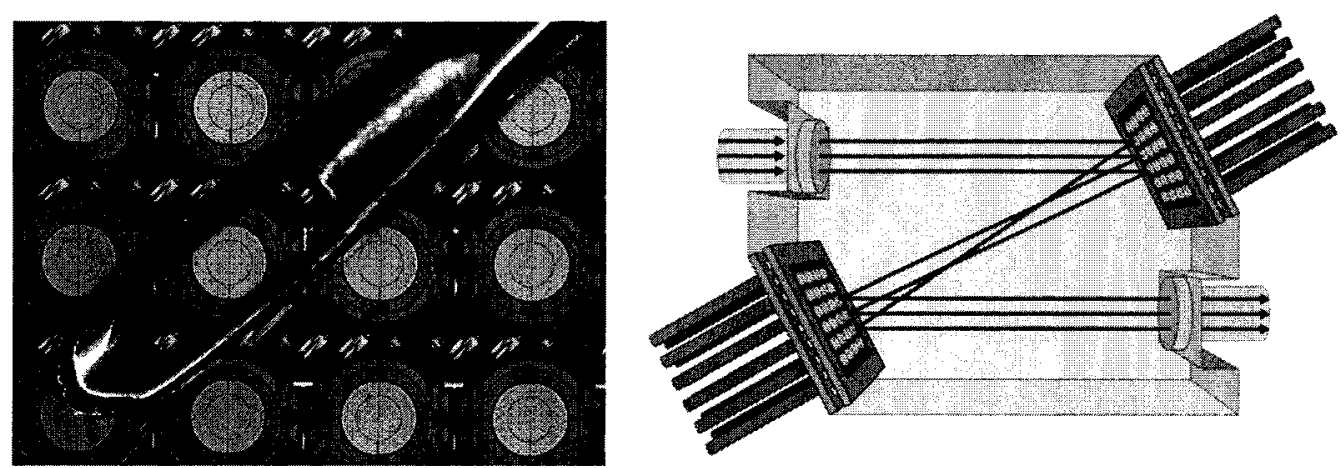

Figure 2.5. Commercial example of an OXC; Lucent's "Lambda Router" [34].

\subsection{Electromagnetic Tuning}

Electromagnetic tuning generates comparatively stronger and longer ranging forces than other methods of actuation. In general, this approach passes current through a micromachined element that generates a magnetic field. The proof mass or structure that will be actuated is normally made from, or is attached to, a ferro-magnetic material. This approach lends itself to tuning the response of an optical device that works with longer wave radiation. For example, Ohnstein et al. $[35,36]$ have developed a large period, high aspect ratio bellows-like grating, shown in figure 2.6, that tunes over a range of $24 \mu \mathrm{m}$ from eight to $32 \mu \mathrm{m}$.

Jin et al. [37] used the strong active force of electromagnetic actuation to strain- 
tune a FBG. Through this method they were able to shift the Bragg wavelength in the communications $\mathrm{C}$ band by $15.7 \mathrm{~nm}$. This shift took place in the time frame of milliseconds.

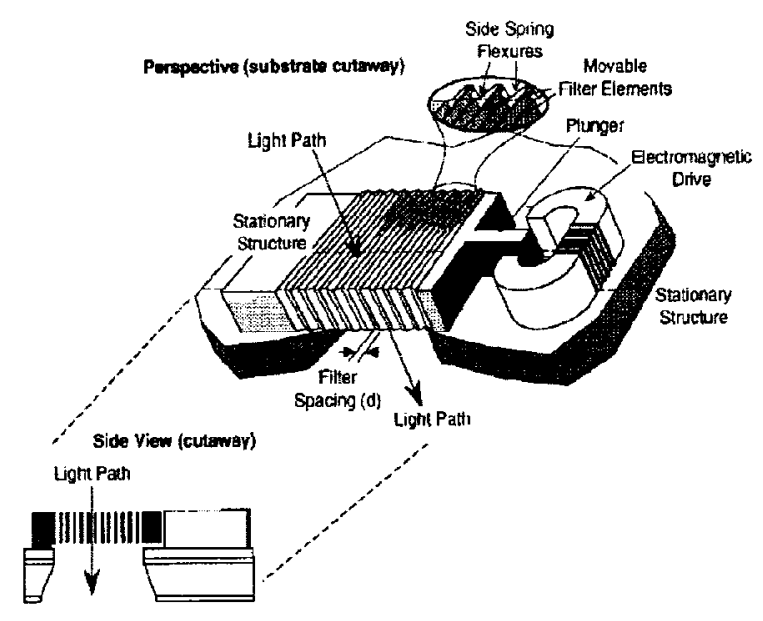

Figure 2.6. Electromagnetically tunable IR filter [36]

\subsection{Shape Memory Alloy Tuning}

Alloy combinations such as $\mathrm{Au} / \mathrm{Cu}, \mathrm{In} / \mathrm{Ti}$, and $\mathrm{Ni} / \mathrm{Ti}$ exhibit a repeatable contraction effect when heated, due to the thermal transition from one crystal phase to another. These materials are known as shape memory alloy (SMA). Thin film Ni/Ti is commonly used as an actuator by passing a current though the alloy to cause a thermal joule effect. SMA actuators are known to generate high displacement and large force to mass ratios under low voltage. Process flow can be challenging however, with common problems including difficult annealing treatments and adhesion problems of thin films. Nevertheless, designs that are compatible with a thermally tunable grating assisted optical waveguide have been attempted [38,39].

A device developed by S. Nagaoka et al. [40] featured a compact tunable FBG filter using a $\mathrm{Ni}$ film heater coated on a FBG and a SMA coil actuator shown in 
figure 2.7. They were able to demonstrate precise wavelength control of under $1 \mathrm{~nm}$ though they do not report the tuning range they were able to achieve.

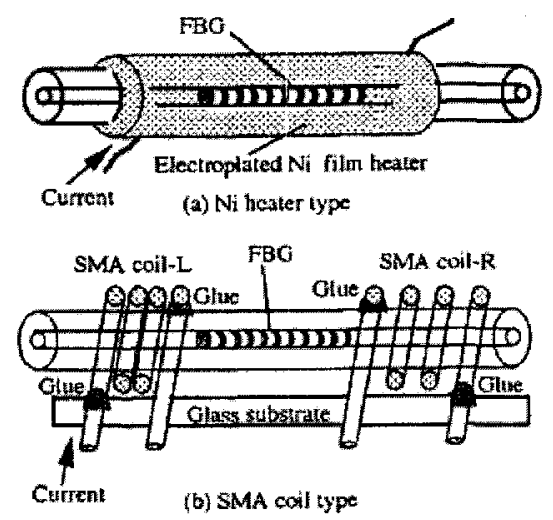

Figure 2.7: Schematic configuration of a SMA tunable FBG filter [40].

\subsection{Thermo-optic Tuning}

The thermo-optic (TO) effect, $d n / d T$, is a material property of all transparent materials including dielectrics, semiconductors and polymers. Table 1 shows the thermo-optic coefficient for a variety of materials [41]. If the coefficient is positive $(d n / d T>0), n$ increases for $\Delta T>0$ (i.e. silicon). If the coefficient is negative $(d n / d T<0), n$ decreases for $\Delta T>0$ (i.e. polymers).

\begin{tabular}{|l|c|c|}
\hline Material & Refractive index & TO coeff. $(\mathbf{d n} / \mathbf{d T}) \mathbf{1 0}^{\mathbf{1}} \mathbf{5}^{\mathbf{}} \mathbf{C}^{\mathbf{- 1}}$ \\
\hline Silica & $1.44-1.47$ & 1.0 \\
\hline Silicon & 3.48 & 18 \\
\hline Indium phosphide & 3.1 & 8.0 \\
\hline Gallium arsenide & 3.37 & 25 \\
\hline Lithium niobate & $2.21,2.14$ (along $\mathrm{x}, \mathrm{z})$ & 1.0 \\
\hline Silicon oxynitride & $1.44-1.99$ & 1.0 \\
\hline Sol-gels & $1.2-1.47$ & 1.0 \\
\hline Polymers & $1.3-1.7$ & -10 to -40 \\
\hline
\end{tabular}

Table 1: Thermo-optic properties of materials used in integrated optics.

Thermo-optic tuning of optical devices makes use of a wide variety of materials 
including silica, semi-conductors, and polymers. While silica waveguides have a comparatively small thermo-optic coefficient, silica on silicon as well as FBG fabrication technology is comparatively mature, particularly in the area of variable optical attenuators. Silicon and GaAs, as well as polymers, have comparatively high TO coefficients and have been useful in DBR, VCSEL, and ROADM designs $[42,43,44,45]$.

S. Yun and J. Lee have developed an in-plane tunable optical filter using the thermo-optic effect of silicon [46]. The device is comprised of a silicon etalon and two DBRs based on silicon/air-gap pairs. Through thermal modulation of the optical thickness of the silicon etalon, they were able to achieve a tuning range of $9 \mathrm{~nm}$; claiming a sensitivity of $0.9 \mathrm{~nm} /{ }^{\circ} \mathrm{C}$. They were careful to note that the optical path length, a product of the refractive index and physical length, is changed predominantly from the thermo-optic effect. They make this claim since their design renders the change in etalon thickness negligible considering the comparatively small effect from the thermal expansion of silicon.

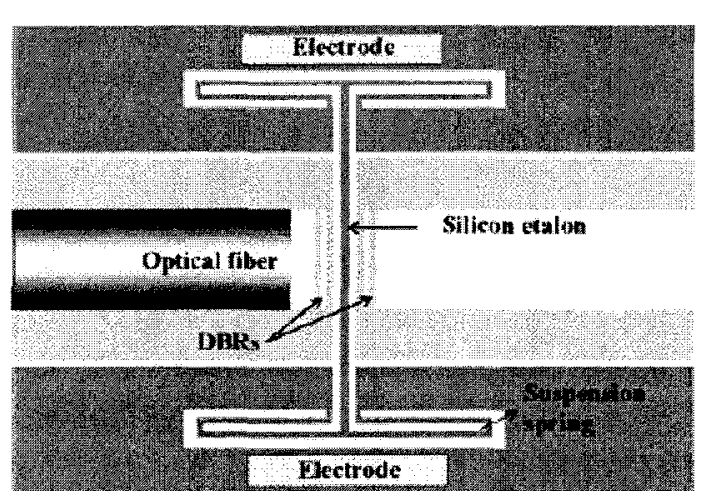

(a)

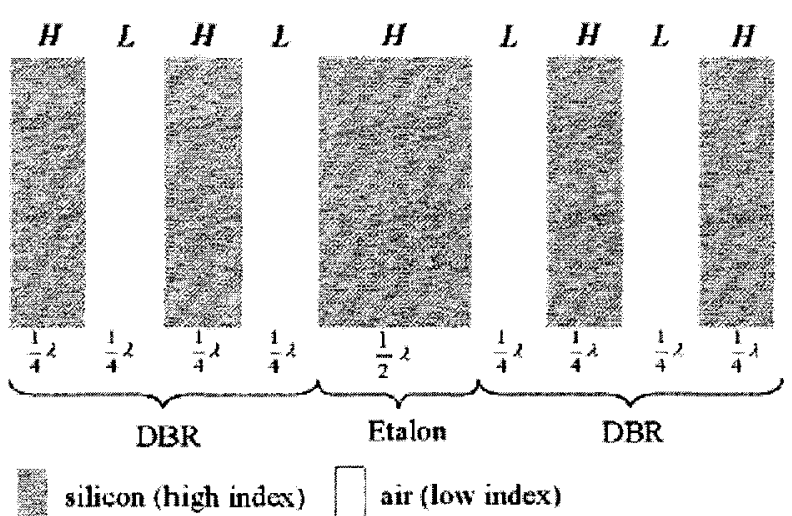

数器 silicon (high index) $\square$ air (low index)

Figure 2.8: In-plane Silicon TOF Horizontally Aligned with an Optical Fibre [46].

Noteworthy in polymer design, L. Eldada et al. [47] have developed a planar polymer Bragg grating $\mathrm{OADM}$ that is tunable over a $20 \mathrm{~nm}$ range with a sensitivity of 
$0.26 \mathrm{~nm} /{ }^{\circ} \mathrm{C}$ and a minimum channel spacing of $75 \mathrm{GHz}$.

\subsection{Thermo-mechanic Tuning}

This method of optical tuning relies on the effect of thermal expansion. The expansion will either manipulate the actuator that produces the tuning effect on the optical element, or directly alter the material the optical element itself is made from. The coefficient of thermal expansion refers to the relative dimensional change in a material that occurs for a given change in temperature. Actuation or tuning occurs as a result of the change in length of the material as it undergoes temperature change.

This effect is not to be confused with the strain-optic effect, in which elastic stress causes a change in the refractive index, since there are many other ways in which actuation can cause stress in a material. In general, since the thermo-elastic effect is so small in comparison to the accompanying thermo-optic effect, it is generally not used on its own to shift the Bragg wavelength of an optical device. It is often used, however, in combination with other material properties to alter the spectral response of a waveguide.

Zhang et al. [48] proposed an add/drop multiplexer based on a polysilicon pitch tunable grating. The design is a kind of thermopile, in which each coupling pair forms one period of the grating. The actuator itself is called a "rhomboid heatuator". When current passes through the four arms of the rhomboid heatuator, the length of the waveguide arms extend to tune the pitch of the grating. A driving current of $10 \mu \mathrm{A}$ causes a temperature change of $200^{\circ} \mathrm{C}$, which in turn actuates the pitch from $13 \mu \mathrm{m}$ to 18.6 $\mu \mathrm{m}$. They did not report the corresponding voltage or power requirements. The tuning response over this range causes a diffraction angle change from $6.8^{\circ}$ to $4.8^{\circ}$ for a 
change in intensity distribution of the \pm 1 order of $1.55 \mu \mathrm{m}$ wavelength light.

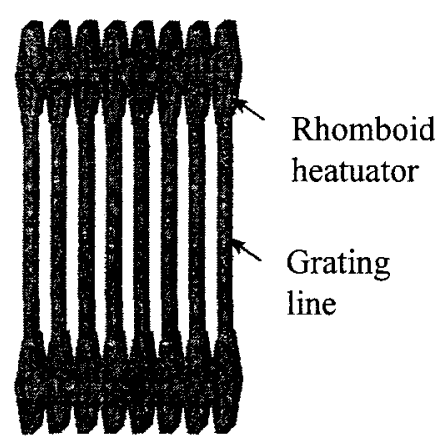

Figure 2.9: Schematic of thermo-elastically pitch-tunable grating filter [48].

In a design reminiscent of the electrostatic device proposed by M. Li et al. [21], the idea of thermal actuation is turned on its head by Amano et al. [49] with a temperature insensitive micromachined AlGaAs-GaAs vertical cavity filter, shown in figure 2.10. This device takes the form of a cantilever in which the suspended beam, and the substrate it hangs over, are made up of layers of AlGaAs and GaAs.

Through a concept they refer to as "thermal inaction", differential thermal expansion in the cantilever renders the thickness of the air gap between the two DBRs thermally stable. This allows the addition of another actuation technique without having to worry about any secondary thermal effect of that actuation technique. This design could be particular useful when used in conjunction with methods of tuning such as SMA or bimetal alloy.

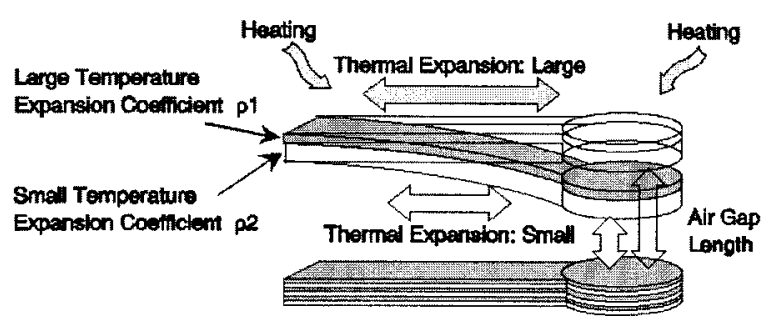

Figure 2.10. Schematic of a thermally insensitive cantilever based vertical cavity filter tuned by thermal expansion [49]. 


\section{A Method for Thermally Tuning the Wavelength of an Optical Filter}

The photonic device described in this thesis uses thermo-optic, photo-elastic and thermomechanic effects; as well as buckling mechanics to control the response of a Bragg filter. These effects are driven by locally heating a suspended waveguide bridge on which the filter itself is integrated. There are three regimes the optical filter operates in. The first regime is the pre-buckle condition. It occurs over the thermal range in which the bridge waveguide lies flat, in plane with the die surface. The thermo-optic and photo-elastic effects combine for a characteristic linear shift in the Bragg filter wavelength per degree Celsius. The second regime involves a discrete shift in the Bragg filter wavelength that is caused when the waveguide bridge buckles at its critical temperature. The discontinuous shift in the Bragg wavelength at this instant is due to a combination of buckling mechanics and the photo-elastic effect. The final regime is the post-buckle condition. In this regime the thermo-mechanical and thermo-optic effects combine to shift the Bragg wavelength, while the photo-elastic effect is no longer present. The Bragg wavelength shift is again linear, but at a greater rate per degree Celsius.

These effects, and their influence on device performance, will be theoretically described in this chapter. This includes the optics of the waveguide and grating filter, the mechanics of the bridge waveguide structure, and the thermal effects on these functional elements due to their material properties.

The optical, thermal, mechanical and buckle theory described here will allow the development of an analytical model. This model will be used in chapter 4 to conduct a design analysis so that the device geometry can be established for fabrication of an SOI- 
based device.

\subsection{Optical Waveguide}

Optical filtering, heating, and actuation produce the functionality of this device, but it is at its core an optical waveguide. Therefore the optical waveguiding theory will be established first to lay the framework for the optical filter, thermal and mechanical elements to follow.

The first factor to consider is the mode property of the waveguide. A waveguide that supports propagation of only a single mode will avoid intermodal effects. Since intermodal dispersion cannot occur in a single mode waveguide, this will maximize the power that can be propagated for a given geometry. Single mode operation will also facilitate the efficient coupling of a single mode fibre to the input and output facets of the waveguide. Therefore, regardless of the geometry the guide takes, it will be designed to be single mode.

Three waveguide geometries were considered. These included channel, strip loaded, and rib waveguides. Common to each waveguide geometry would be a contact cut etch in the device layer of the SOI to define an open rectangular area on each side of the waveguide. After etching through the silicon to expose the BOX layer, a surface micromachining process will release the waveguide from the BOX so it is suspended in air as a waveguide bridge. Each design will also require accommodation for an integrated heater. In the case of the channel and strip loaded designs, the heater element would not only provide thermal tuning, but waveguiding as well. 


\subsubsection{Channel Waveguide}

The channel waveguide uses adjacent indices of refraction that are less than the core index to define the waveguide. A longitudinal cross-section is depicted in figure 3.1. The vertical confinement is due to the index contrast between the silicon waveguide and air. The lateral confinement would come from the index contrast produced by diffusion of implanted ions, typically phosphorous or boron, into the suspended waveguide bridge.

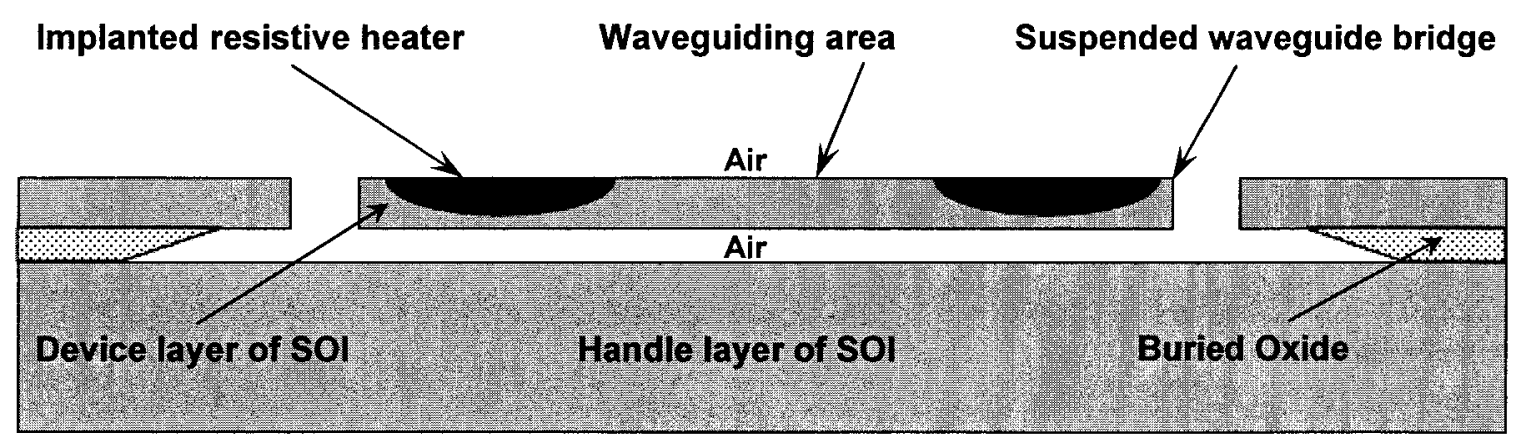

Figure 3.1. Channel waveguide example.

The change in index of refraction that free carriers cause has been explored by Soref and Bennett [50]. They report that a phosphorus carrier density of $10^{20} \mathrm{~cm}^{-3}$ will cause an index change of -0.1 . For guiding to occur, the waveguiding core is typically surrounded on all sides by a confining medium of lower index of refraction. Since $\Delta n$ is negative, it follows that the areas to either side of the waveguide must receive the phosphorus implantation rather than the core itself. By implanting and then diffusing ions into the device layer of the SOI, a pn junction is created that defines the dimensions of the lateral confinement strips.

The negative index change is also fortuitous, since implanting silicon with phosphorus also causes a substantial attenuation effect to the light on the order of 
$2290 \mathrm{~cm}^{-1}$ [50]. The mode of propagation will be in the lower loss undoped centre region. There would, however, be a loss associated with the boundary between the doped and undoped silicon. Discontinuities in the boundary would contribute to scattering loss along the waveguide length.

If there is effective junction isolation between the p-type isolation strips and the n-type device layer silicon that contains them, electrical leakage to the waveguide bridge itself can be ignored, and the isolation strips will double as self-heating silicon resistors. The resistive heaters would provide thermal tuning by passing a current through them.

\subsubsection{Strip-loaded Waveguide}

In this design metallic electrodes forming the integrated heaters would also be used to produce optical striplines. When considering an analysis of a strip loaded waveguide, the location of the stripline will be determined by the index contrast between the waveguiding material and the material the strip is made from.

In the case where the cladding stripline is a dielectric material of lesser index than the waveguide, the strip is deposited on top of the waveguide, creating an index larger than the unclad regions adjacent to it. In the case where the loading strip is metal, the effective index in the region beneath it is smaller than the effective index in the core region where there is no metal loading strip.

Therefore, as in the case of the channel waveguide, the waveguiding channel is defined laterally by strips of a metallic material deposited on either side. Confinement in the vertical direction would again come from the release of the bridge waveguide, exposing the top and bottom of the waveguide to air. A cross-section of this waveguide type is shown in figure 3.2. 
This option would not suffer from the sidewall roughness that we shall see in the case of the rib design, or loss due to confinement by ion implantation boundaries in the case of the channel waveguide design. There would, however, be optical power loss due to absorption of the photons at the metal interface. The primary disadvantage to this approach would be the optical loss on the metal interface of the heater strips, since metal is not a perfect conductor at optical frequencies [51].

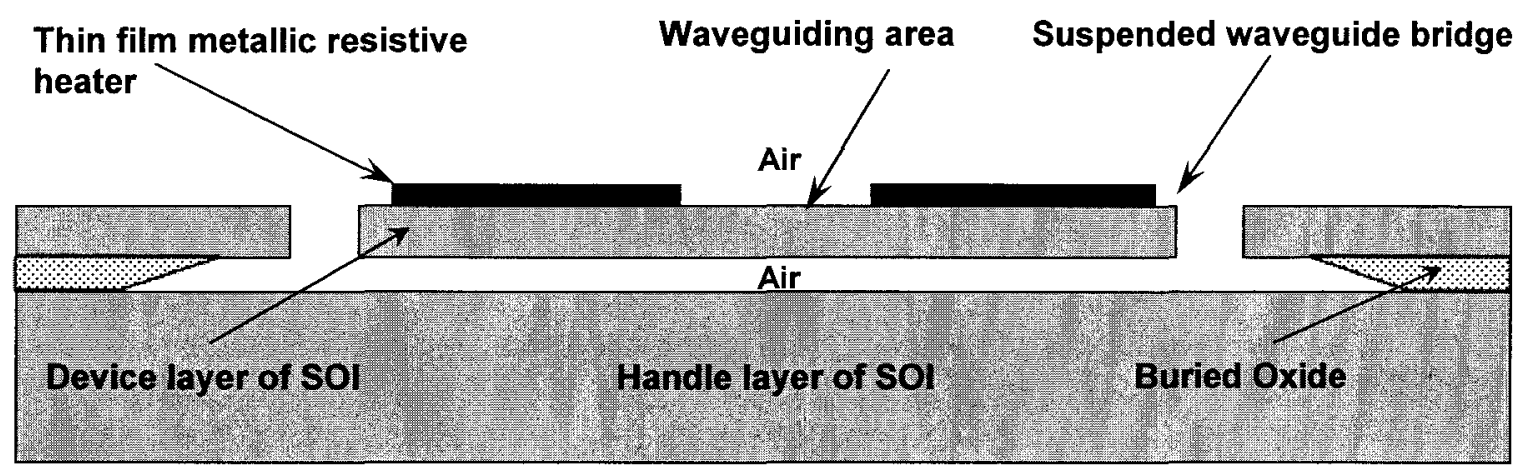

Figure 3.2: Metal strip-loaded waveguide

Loss could be reduced by processing a thin buffer layer of oxide between the heater/waveguiding metal strips and the silicon, producing a buffered metal waveguide [52]. This would introduce a difficulty in the sacrificial release of the waveguide bridge, however, since the same step that removed the BOX layer between the device layer and handle layer silicon would also attack this buffer oxide. To analyse the channel and striploaded waveguide types, in order to calculate the effective index and propagation constant, methods developed by Marcatili [53] and Furuta [54] would be employed.

\subsubsection{Rib Waveguide}

This design geometry makes use of an etch process to create two trenches in the guiding silicon slab of an SOI wafer. In conjunction with the thickness of the device layer of the SOI, the separation of the trenches becomes the rib width that defines the 
core of the waveguide. A cross-section of the rib type waveguide in SOI is shown in figure 3.3.

As before, the contrast between the indices of air and silicon will confine the optical mode to the rib waveguide in the vertical direction. In the lateral direction, the effective index on either side of the rib waveguide will be less than the index of the full thickness of the rib, providing confinement in the lateral direction. The main source of loss with this waveguide type is the radiative loss from the sidewall roughness of the rib itself.

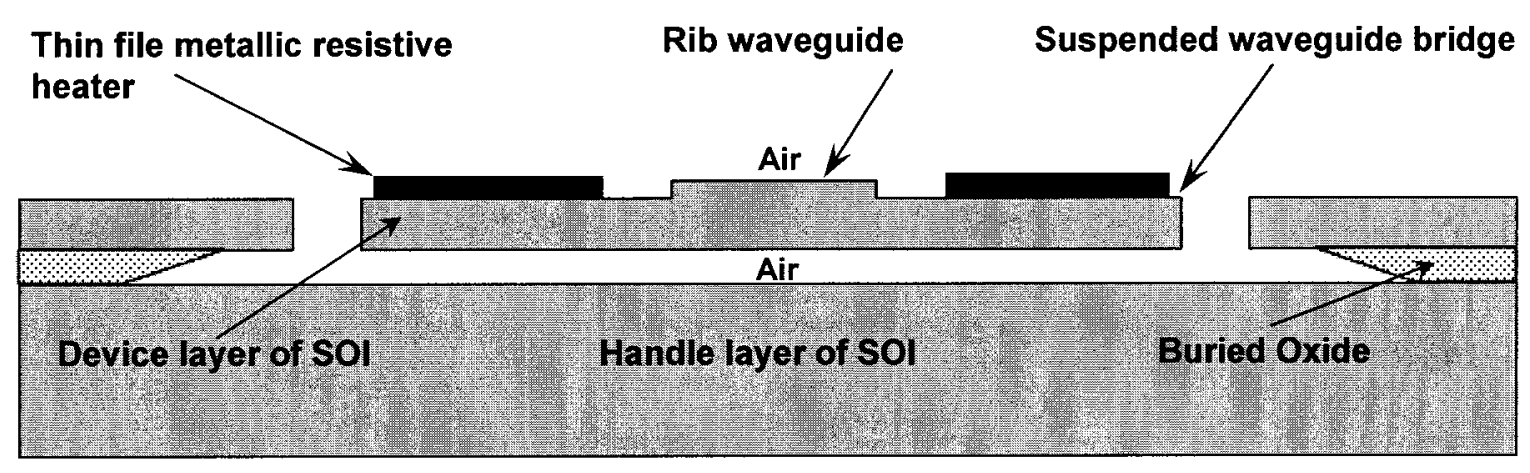

Figure 3.3. Suspended rib waveguide on SOI.

Since the rib provides lateral confinement in the waveguide, there is freedom to use either implanted or metallic heaters to thermally tune the device. With these elements moved some distance from the rib itself, loss associated from either element will also be reduced.

Another benefit of lateral mode confinement without the use of an integrated heating strip is that the suspended waveguide filter could be thermally tuned and actuated through the use of an external heat source. This allows for extra flexibility in the design and fabrication cycle since a device could be mechanically and optically characterised without an integrated heater. 
The width and height of the rib, and the index contrast it provides, defines the waveguiding properties of the rib waveguide in relation to the thickness of the bridge waveguide itself [55]. This concept will prove to be of critical importance in the design process when it will become apparent that the bridge thickness plays an integral role in the mechanical and therefore optical performance of this filter.

The final important difference between the other two waveguiding types and the rib waveguide is mechanical rather than optical. The cross-section of the channel and strip loaded waveguides is rectangular (or prismatic), while the rib waveguide crosssection is a " $\mathrm{T}$ " shaped beam. While the rib is a small surface perturbation in comparison to the slab that forms the waveguide bridge, it will still reduce the moment of inertia of the beam. This will translate to a lower critical buckle temperature.

The rib waveguide is difficult to analyse using Marcatili's method because of the division of the waveguide geometry. Analytically, the effective index method (EIM) can be used to calculate the modality and propagation constant of this structure [56].

\subsubsection{Comparing waveguide types}

The design strengths, weaknesses and process challenges of each waveguide type are summarized in table 2. An existing rib waveguide and Bragg filter mask, and etch process, were developed at Carleton University's Microelectronics Fabrication Laboratory (MFL) [57]. These existing device elements could be adapted to the rib waveguide filter proof-of-concept process flow that would be developed for the first design iteration of the device. This benefit, combined with a more flexible method of thermal actuation, makes the rib waveguide the most favourable design to move forward with. 


\begin{tabular}{|l|l|l|l|}
\hline \multicolumn{1}{|c|}{ Waveguide } & \multicolumn{1}{c|}{ Channel } & Strip Loaded & \multicolumn{1}{c|}{ Rib } \\
\hline $\begin{array}{l}\text { Process } \\
\text { Challenges }\end{array}$ & $\begin{array}{l}\text { - Lateral phosphorus } \\
\text { diffusion must be } \\
\text { controlled }\end{array}$ & $\begin{array}{l}\text { - Delamination of } \\
\text { metallic strip line }\end{array}$ & $\begin{array}{l}\text { - Sidewall roughness } \\
\text { - Delamination of metallic } \\
\text { strip line }\end{array}$ \\
\hline Strengths & $\begin{array}{c}\text { - Less sidewall } \\
\text { imperfection than rib }\end{array}$ & $\begin{array}{l}\text { - Can test without integrated } \\
\text { heaters } \\
\text { - Least process steps }\end{array}$ & $\begin{array}{l}\text { - Building on developed } \\
\text { waveguide process } \\
\text { - Heating elements separated } \\
\text { from WG core }\end{array}$ \\
\hline Weakness & $\begin{array}{c}\text { - Most complicated } \\
\text { process }\end{array}$ & $\begin{array}{c}\text { - May not be compatible } \\
\text { with HF bridge release }\end{array}$ & - Sidewall roughness in rib \\
\hline
\end{tabular}

Table 2: Summary of waveguide designs with process challenges, strengths and weaknesses.

Figure 3.4 shows the top section and cross view of a rib waveguide, where $W$ is the rib waveguide width and $H$ is the rib waveguide thickness, equal to the thickness of the device layer of the SOI. The thickness of the slab waveguide is $h$. The rib waveguide height is the difference between $H$ and $h$. The top view is used to show the guiding characteristics of an equivalent slab guide in order to predict the guiding properties of the rib guide in the y-direction. Index $n_{\text {eff }(h)}$ is the effective index of the slab portion of the waveguide and index $n_{e f f(H)}$ is the effective index of the rib waveguide [58].

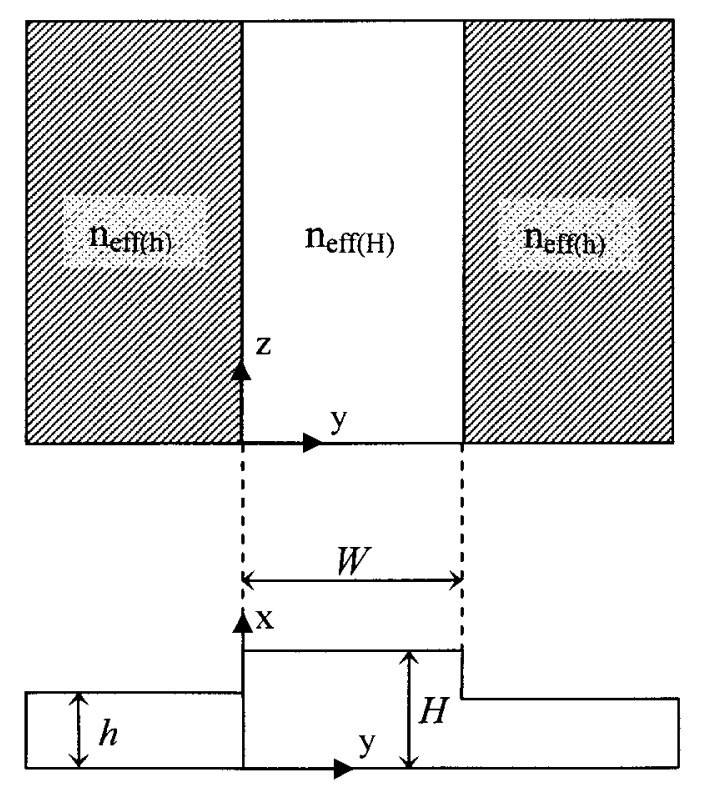

Figure 3.4. Top view and cross-section of a rib waveguide for EIM. 
The boundary condition is such that $\mathrm{E}_{\mathrm{z}}$ is continuous at $\mathrm{y}=0$ and $\mathrm{W}$. In general, the effective index, $n_{\text {eff }}$, is expressed as:

$$
n_{e f f}=\frac{\beta}{k}
$$

where propagation constant in the $z$ direction (or longitudinal wavenumber) is $\beta$ and the wavenumber $k=2 \pi / \lambda$.

The dispersion equation is [59]:

$$
u \tan (u)=\frac{n_{e f f(H)}^{2}}{n_{e f f(h)}^{2}} w
$$

where $u$ and $w$ are transverse wavenumbers:

$$
u=\frac{k W}{2} \sqrt{n_{e f f(H)}^{2}-\left(\frac{\beta}{k}\right)^{2}}
$$

and

$$
w=\frac{k W}{2} \sqrt{\left(\frac{\beta}{k}\right)^{2}-n_{\text {eff }(h)}^{2}}
$$

If the transcendental equation 3.2 were solved graphically, the result is a range of discrete allowed values of $\beta$ corresponding to the allowed modes. In chapter 4 , software using numeric methods will conduct an analysis on the rib waveguide design to determine $\beta$ over a range of wavelengths.

\subsection{Bragg Grating Filter}

The Bragg grating filter is etched into the waveguide rib so that light can be directed to it. The wavelength of maximum reflectivity, $\lambda_{B}$, is characterised by the Bragg equation: 


$$
\lambda_{B}=2 n_{e f f} \Lambda
$$

This wavelength occurs as a result of forward and backward propagating mode coupling, and is determined from the mode effective index in one period of the grating, $n_{\text {eff }}$, and the Bragg grating period itself, $\Lambda$, as depicted in figure 3.5.

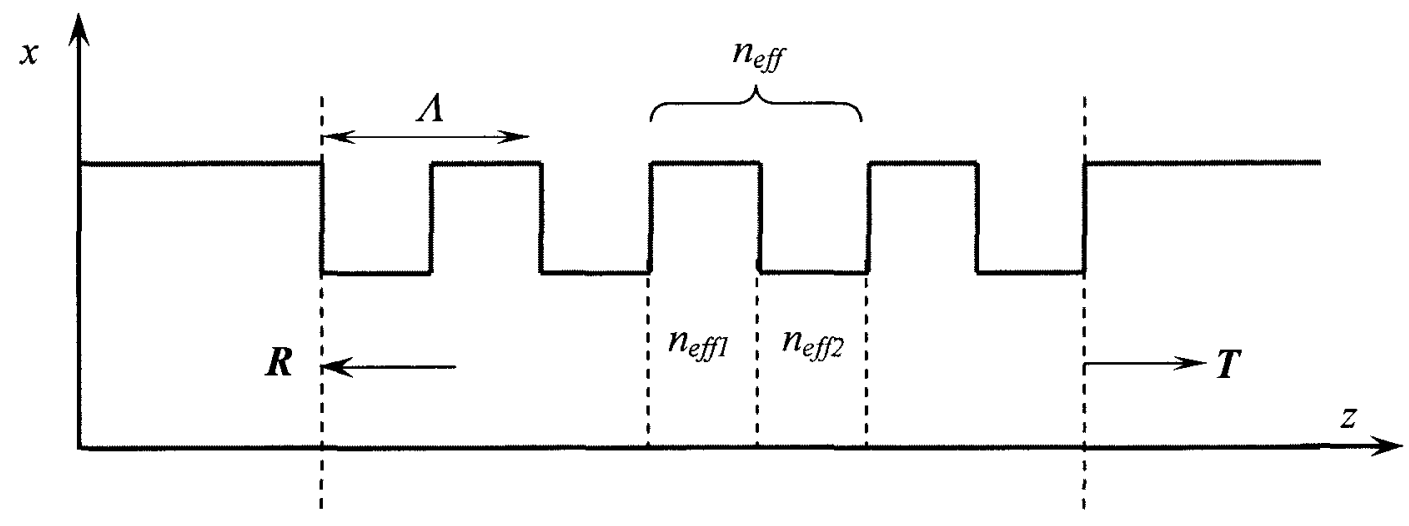

Figure 3.5. Lengthwise cross-section of Bragg grating with dimensions.

The Bragg equation only indicates the wavelength at which maximum reflectance occurs. Also indicated on the figure is the reflectance, $R$, and transmittance, $T$. These values refer to the power ratio of the reflected light to incident light, and transmitted light to incident light, respectively, at a specific wavelength from the centre Bragg peak.

Equation 3.5 is the foundation upon which this thermally tuned suspended waveguide filter will be analysed. The device proposed here will change the Bragg wavelength by changing both the effective index of the rib waveguide that the Bragg grating has been integrated, and the pitch of the Bragg grating. This relationship can be informally stated by expanding equation 3.5 into the following phenomenological equation:

$$
\Delta \lambda_{B}=2 n_{e f f} \Delta \Lambda+2 \Delta n_{e f f} \Lambda
$$

The equation will be more formally expanded in the next chapter. 
It follows from equation 3.6 that the shift in wavelength at which minimum transmittance occurs will be affected by any thermal or stress based change in the grating index due to thermo-optic or photo-elastic effects; or any thermal or mechanical based change in the pitch of the grating due to thermal expansion or the buckling mode shape.

While this relationship is important to determine the wavelength of the Bragg peak for a given set of thermal and mechanical based operational parameters, a more rigorous definition of the grating transmittance is necessary to be able to model the shape and strength of the Bragg response. To determine the wavelength of the peak requires equation 3.5 , but to get a sense of how to maximize the filter strength and minimize the passband bandwidth at full-width half-maximum (FWHM), $\delta \lambda_{3 d B}$, a different theoretical model is required. The nature of the forward and backward propagating mode coupling of the grating must be determined.

\subsubsection{Coupling Coefficient}

Yariv has shown [60] that for a rectangular grating, depicted in figure 3.6, the coupling coefficient, $\kappa$, of the filter (or selective mirror) can be characterised by the following formula:

$$
\kappa=\frac{2 \pi^{2}}{3 l \lambda} \frac{n_{\text {core }}^{2}-n_{\text {air }}^{2}}{n_{\text {core }}}\left(\frac{a}{t_{g}}\right)^{3}\left[1+\frac{3(\lambda / a)}{2 \pi\left(n_{\text {core }}^{2}-n_{\text {air }}^{2}\right)^{1 / 2}}+\frac{3(\lambda / a)^{2}}{4 \pi^{2}\left(n_{\text {core }}^{2}-n_{\text {air }}^{2}\right)}\right]
$$

where $n_{\text {core }}$ is the effective core index of the rib waveguide, $n_{\text {air }}$ is the index of refraction of air, $l$ is the order of harmonic coupling, $\lambda$ is the wavelength coupled into the waveguide, $a$ is the grating depth, and $t_{g}$ is the waveguide thickness. Also labelled in figure 3.6 is the grating duty cycle, $D$, which is the ratio between the grating width, $b$, and the grating pitch, $\Lambda$; or $D=b / \Lambda$. 
The order of the harmonic response used to calculate the coupling coefficient $\kappa$ is given by:

$$
l \cong \frac{\beta_{m} \Lambda}{\pi}
$$

where $\beta_{m}$ is the propagation constant of the particular mode $(m)$ under consideration, and $\Lambda$ is the grating period. For the first order $(l=1)$ the reflection wavelength, which is $\lambda_{B}$, occurs as a result of forward and backward propagating mode coupling of the fundamental mode travelling in the $\pm z$ direction.

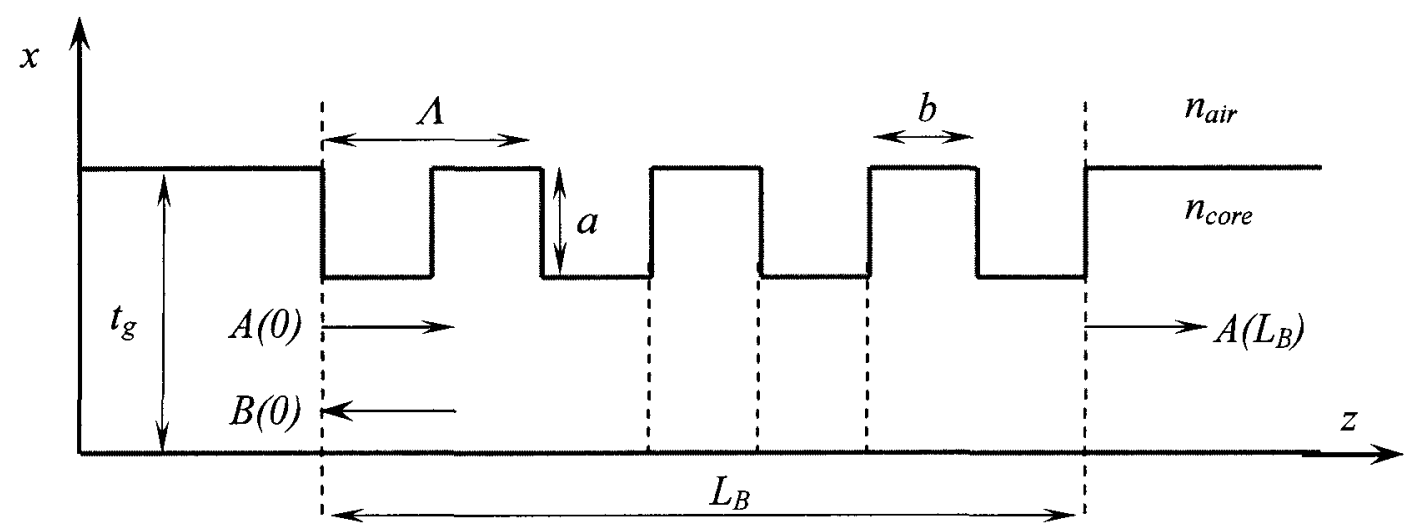

Figure 3.6. Dimensions of a grating used for the coupled mode solution of the Bragg filter spectral response.

The solution of the coupled mode equation of the waveguide as a function of distance, $z$, which is the distance the incident wave travels along the grating, is given by the mode reflectivity equation 3.9 [61]. A(z) describes the forward propagating wave and $B(z)$ describes the backward propagating wave such that:

$$
A(z) e^{i \beta z}=B(0) \frac{i \kappa \exp \left(i \beta_{0} z\right)}{-\Delta \beta \sinh \left(S L_{B}\right)+i S \cosh \left(S L_{B}\right)} \cdot \sinh \left[S\left(z-L_{B}\right)\right]
$$

where $L_{B}$ is the length of grating, $\beta_{0}$ is the lowest order mode propagation constant of the waveguide at a given wavelength, and $\beta$ is the propagation constant of the waveguide at a 
given wavelength, where the Bragg filter has been integrated.

Additionally, $S$ is a variation of the coupling constant, and is calculated thus:

$$
S=\sqrt{\kappa^{2}-(\Delta \beta)^{2}}
$$

with

$$
\Delta \beta \equiv \beta-\beta_{0}
$$

and

$$
\beta_{0} \equiv \frac{\pi l}{\Lambda}
$$

where $\Delta \beta$ is the detuning value.

In addition to providing a solution for the reflectivity versus the deviation from the Bragg condition, the mode reflectivity equation can also be used to calculate the same spectral shift of the Bragg reflector that the expansion of equation 3.6 provides in order to compare the results of the two. In the following sections of this chapter the Bragg wavelength equation, 3.6, will be deconstructed into its thermo-optic, thermo-mechanic, and photo-elastic components and then combined in chapter 4 for analysis and design The resulting effective index and grating pitch changes will be used to compute the shift in the Bragg wavelength response over a range of temperatures for a specific device geometry.

\subsection{Heaters}

The waveguide bridge that contains the Bragg grating must be heated locally. If the entire die were heated, thermal expansion over the surface would prevent differential expansion of the bridge from its anchor points on the die. Two approaches were 
considered to achieve the thermal differential required to actuate the suspended waveguide bridge and tune the filter it contained. The first involved integrating selfheating resistors onto the bridge, on either side of the rib waveguide. The second entailed the application of an external localised heat source to the bridge instead of from the integrated heaters.

Heating elements can be integrated either as implantation and diffusion of a resistor into the silicon substrate, or deposited on the silicon surface as a thin film metallic resistor. A flow of electrical current through the resistor is converted to heat via Joule heating. The resistance of a heater is the result of its dimensions and material properties, and can be calculated from the following equation:

$$
R=\frac{\rho L}{A}
$$

where $\rho$ is the resistivity; and the dimensions of the resistor are determined by its crosssectional area, $A$, and its length, $L$. The resistivity of the heater material can be obtained from a material properties handbook if the material is of sufficient purity, or measured experimentally.

The temperature increase due to Joule heating is linear over a characteristic temperature range that depends on the resistor material. Resistance is known to change as temperature changes. This material property is called the temperature coefficient of resistance (TCR). Once the resistance of a heater has been confirmed experimentally at a reference temperature (room temperature for instance), the temperature of the heater can be calculated if the resistance is known at that current. This can be accomplished by measuring the voltage across the resistor for that current, and using Ohm's law to calculate the resistance. 
The following equation incorporates these effects:

$$
R_{T}=R_{0}\left[1+\tau\left(T-T_{0}\right)\right]
$$

where $R_{T}$ is the resistance at temperature $T, R_{0}$ is the resistance at reference temperature $T_{0}$, and $\tau$ is the TCR of the material the resistor is composed of. Equation 3.14 can be rearranged in terms of temperature as follows:

$$
T=\frac{R_{T}-R_{0}+\tau R_{0} T_{0}}{\tau R_{0}}
$$

This relationship will be used when thermally characterising the optical performance of the device using integrated heaters.

The second technique for thermal actuation involves focusing the output of an external laser diode (LD) onto the bridge so it can be heated locally in comparison to the surrounding surface layer at its anchor points. This technique requires the LD to operate in its linear region over the thermal region the device is characterised. The LD must also operate at a wavelength for which the silicon is highly absorptive.

The self-heating resistor, hereafter referred to as a heater, is the preferred solution for a variety of reasons. An external heater requires a more complicated experimental setup. Measuring the thermal effects on the device using an integrated heater is also more direct. An external heater requires additional experimentation to translate a shift due to the laser power to a shift due to the thermal effect of the laser power.

During the design, fabrication, and characterisation process for the devices that were developed, both heating techniques were explored. Although the integrated heater approach was preferred, external heating proved invaluable for both rapid bridge buckle characterisation as well as a contingency when the integrated heaters were not available. 


\subsection{Thermo-optic Effect}

The thermo-optic effect describes the temperature dependency of the refractive index. The effect is a combination of the change in index as the material's polarisability and density changes with respect to temperature. Polarisability is a material property that quantifies the ease of distortion of the electron cloud in the atoms of a crystal by an electric field. An increase in polarisability will increase the refractive index.

The coefficient of thermal expansion, discussed in section 3.5 , is a material property indicative of the extent to which a material's dimensions change upon heating Since the number of atoms or molecules in the material remain constant, the density of the material changes as the volume (and temperature) changes. In general, a decrease in density will decrease the refractive index [62].

As the temperature changes, the change in polarisability and density of the material competes to determine the physical value of the refractive index. The dominant coefficient determines whether the thermo-optic coefficient will be positive or negative as a result.

The following equation describes this effect [63]:

$$
n=\sqrt{1+\frac{N \phi}{\varepsilon_{0}}}
$$

where $n$ is the refractive index, $N$ is the material density, $\phi$ is the material polarisability, and $\varepsilon_{o}$ is the permittivity of free space. When $N$ and $\phi$ change as a result of the change in temperature, $T$, the resulting $\mathrm{dn} / \mathrm{dT}$ is called the thermo-optic coefficient (TOC), and is represented by the symbol $\xi$ in this thesis. TOC is a material property and for silicon it is positive. At room temperature $\xi=1.75 \times 10^{-4}{ }^{\circ} \mathrm{C}^{-1}[64]$. 


\subsection{Thermo-mechanic Effect}

The thermo-mechanical effect describes the temperature dependency of the linear dimension of a material. As with the thermo-optic effect, a material property coefficient relates this dimensional change to a change in the material temperature. In this case the change, or deformation to be exact, is called the strain, $\varepsilon$. Strain is the ratio of change in linear dimension, $\Delta L$, to its original dimension, $L_{0}$, as shown in equation 3.17. Although the symbol, $\varepsilon$, can also refer to the dielectric permittivity, unless otherwise stated it refers to strain in this thesis.

$$
\varepsilon=\frac{\Delta L}{L_{0}}
$$

When deformation occurs as a result of the change in temperature, $\varepsilon / \mathrm{dT}$ is called the linear thermal expansion coefficient (TEC). This material property is represented by the symbol, $\alpha$. For silicon at room temperature $\left(22^{\circ} \mathrm{C}\right)$ it is $\alpha=2.53 \times 10^{-6}{ }^{\circ} \mathrm{C}^{-1}[65]$.

The TEC relates strain to temperature change in the following equation:

$$
\varepsilon=\alpha \Delta T
$$

As deformation occurs due to this thermal expansion, the material will have a characteristic stiffness, or capacity to resist deformation. This material property is called the modulus of elasticity, and is represented by the symbol $E$. Although $E$ can also refer to the electric field, unless otherwise stated it refers to the modulus of elasticity in this thesis.

The most general modulus of elasticity is a bulk isotropic number, which is the material's resistance to being compressed from all sides. The bulk modulus of elasticity for silicon is $98 \mathrm{GPa}$ [66]. If deformation of the structure takes place in a specific 
direction, the bulk modulus is the least accurate material property to use when analysing the mechanical behaviour of the device. Young's modulus, the capacity of the material to resist deformation in a linear direction, is generally taken as $130 \mathrm{GPa}$ for silicon [67]. When the exact crystallographic direction of deformation is known, an even more accurate value for $E$ can be derived. The material used to fabricate the suspended waveguide bridge will be single crystal silicon, for which the crystallographic orientation will be known. Therefore the crystallographic representation of $E$ can be derived for a better understanding of the mechanical and optical behaviour of the buckling beam.

When a material's resistance to being deformed is combined with a deformation, the force applied per unit area to cause that deformation is referred to as the stress, $\sigma$. The stress is related to strain via the modulus of elasticity in the following equation:

$$
\sigma=E \varepsilon
$$

Equations 3.18 and 3.19 are fundamental relationships that will be used in conjunction with buckling mechanics to determine the temperature at which the waveguide bridge will buckle. This critical temperature, along with the accompanying thermo-optic, thermo-mechanic, and photo-elastic effects, will determine the thermomechano-optical change in the Bragg wavelength response of the waveguide filter.

A strain tensor is used to describe the change in cubic structure of the silicon lattice due to deformation by thermal expansion. The resulting stress tensor consists of normal strain components $\varepsilon_{\mathrm{x}}, \varepsilon_{\mathrm{y}}$ and $\varepsilon_{\mathrm{z}}$ and shear strain components $\gamma_{\mathrm{xy}}, \gamma_{\mathrm{xz}}$ and $\gamma_{\mathrm{yz}}$. The stress tensor describes the pressure that is applied to the silicon crystal. It includes normal stresses $\sigma_{\mathrm{x}}, \sigma_{\mathrm{y}}$ and $\sigma_{\mathrm{z}}$ and shear stresses $\tau_{\mathrm{yz}}, \tau_{\mathrm{zx}}$ and $\tau_{\mathrm{xy}}$. Recalling equation 3.19, the stress and strain are related via the modulus of elasticity of silicon. As a result of the 
cubic structure of silicon, the crystallographic representation of $E$ in the $\langle 100\rangle$ axes has the stiffness coefficients $C_{11}, C_{12}$, and $C_{44}$. The relationship between stress and strain can be expressed in matrix form with equation 3.20. The values for $C$ are as follows: $C_{I I}=165.6 \times 10^{9} \mathrm{~Pa}, C_{I 2}=63.98 \times 10^{9} \mathrm{~Pa}$ and $C_{44}=79.51 \times 10^{9} \mathrm{~Pa}[66]$.

$$
\left[\begin{array}{c}
\sigma_{x} \\
\sigma_{y} \\
\sigma_{z} \\
\tau_{y z} \\
\tau_{x z} \\
\tau_{x y}
\end{array}\right]=\left[\begin{array}{cccccc}
C_{11} & C_{12} & C_{12} & 0 & 0 & 0 \\
C_{12} & C_{11} & C_{12} & 0 & 0 & 0 \\
C_{12} & C_{12} & C_{11} & 0 & 0 & 0 \\
0 & 0 & 0 & C_{44} & 0 & 0 \\
0 & 0 & 0 & 0 & C_{44} & 0 \\
0 & 0 & 0 & 0 & 0 & C_{44}
\end{array}\right]\left[\begin{array}{c}
\varepsilon_{x} \\
\varepsilon_{y} \\
\varepsilon_{z} \\
\gamma_{y z} \\
\gamma_{z x} \\
\gamma_{x y}
\end{array}\right]
$$

The device layer of single crystal SOI used in this research has a standard crystallographic orientation that includes the (001) face with a flat ground to indicate the (110) face. The lithographic masks that define the features of the device will be oriented to this flat. Therefore the axis of the suspended waveguide will be parallel to the [110] direction of the silicon crystal. Figure 3.7 shows the orientation of a silicon wafer within cubic hexoctahedral single crystal silicon [68], which in turn shows the crystallographic orientation of the waveguide bridge structure on the wafer itself. The crystallographic orientation of the waveguide bridge will also impact the influence of the photo-elastic effect. This will be covered in section 3.6.

The general formula for determining the modulus of elasticity in an arbitrary crystallographic direction is [69]:

$$
\frac{1}{E}=S_{11}-2\left(S_{11}-S_{12}-\frac{1}{2} S_{44}\right)\left(l_{1}^{2} l_{2}^{2}+l_{2}^{2} l_{3}^{2}+l_{1}^{2} l_{3}^{2}\right)
$$

where $l_{i}$ are the directional cosines for $l$; and $S_{i j}$ are the compliance coefficients of the material, and the inverse of matrix $C_{i j}$. The values for $S$ are: $S_{I 1}=76.8 \times 10^{-13} \mathrm{~Pa}^{-1}$, 
$S_{12}=-21.4 \times 10^{-13} \mathrm{~Pa}^{-1}$ and $S_{44}=126 \times 10^{-13} \mathrm{~Pa}^{-1}$ [66]. The modulus of elasticity for single crystal silicon in the $\langle 110\rangle$ direction is $\mathrm{E}_{\langle 110\rangle}=168.0 \mathrm{GPa}$.

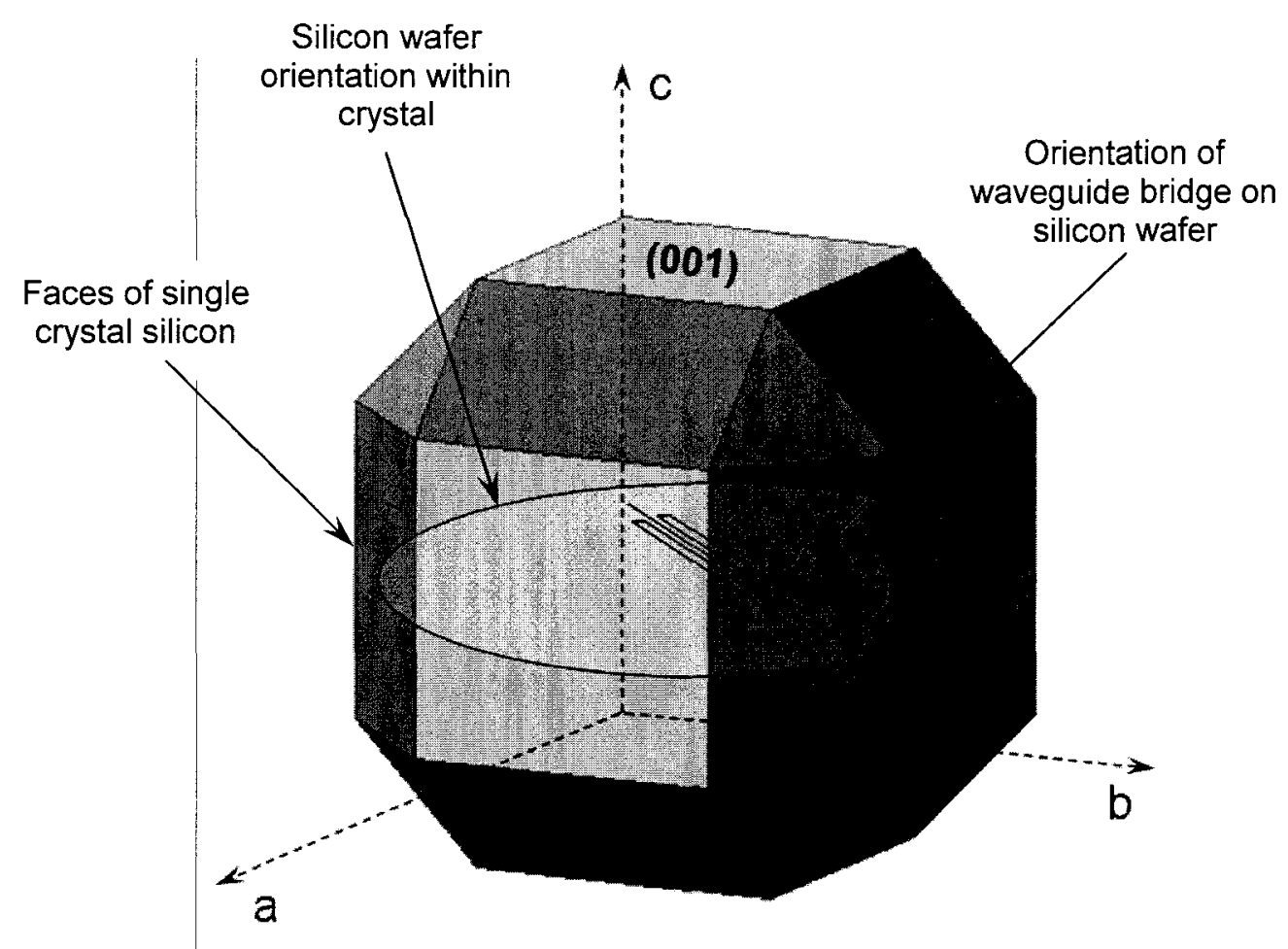

Figure 3.7. Orientation of waveguide bridge within the crystallographic planes of single crystal silicon to help determine the mechanical properties of the bridge [68].

The fixed-fixed beam that forms the waveguide bridge is much longer than it is thick or wide, and the sides and surfaces of the beam are free to expand without restriction, so the uniaxial stress that will occur along the axis of the beam need only be considered [69]. Equations 3.18 and 3.19 can be combined into a relationship that will take into account the crystallographic orientation of the waveguide bridge when calculating the stress within the bridge due to constrained axial thermal expansion in the $\langle 110\rangle$ direction:

$$
\sigma_{\langle 110\rangle}=\alpha \Delta T E_{\langle 110\rangle}
$$




\subsection{Photo-elastic Effect}

The change in refractive index caused by stress is the photo-elastic effect. When a crystal is subject to a stress field, the resulting photo-elastic effect is dependant on the orientation of the optical indicatrix to the crystallographic axes, and the mechanical effects they represent. Overlaying these two axes results in the optical orientation of the crystal. In order to relate the optical property of the photo-elastic effect to the mechanical effect of the thermally stressed beam, the optical orientation of silicon is important to consider.

Silicon belongs to the isometric crystal system, and has the highest order of symetry among the seven crystal systems. Its class is cubic hexocahedral as is shown in figure 3.8. Crystallographic axes $[\mathbf{a}, \mathbf{b}, \mathbf{c}]$ and tensor reporting frame axes $[\mathbf{X}, \mathbf{Y}, \mathbf{Z}]$ for silicon are designated according to IEEE/ANSI STD 176-1987, which equates $\mathrm{X}=\mathrm{a}=\mathrm{z}$, $\mathrm{Y}=\mathrm{b}=\mathrm{x}$, and $\mathrm{Z}=\mathrm{c}=\mathrm{y}$, relative to principal optical axes $[\mathbf{x}, \mathbf{y}, \mathbf{z}]$.

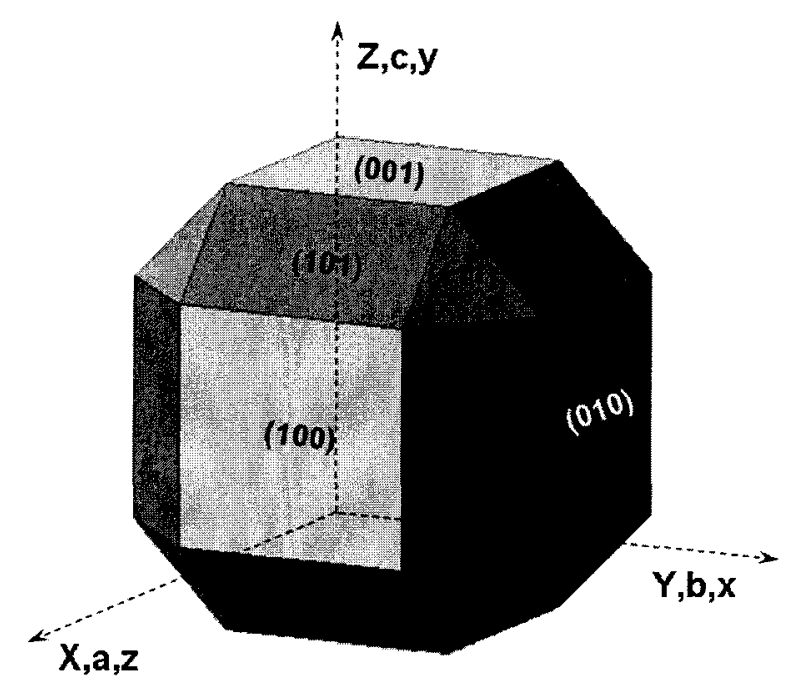

Figure 3.8. Cubic hexoctahedral silicon showing the alignment of the optical and crystallographic axis in an optical orientation plot. 
Since silicon has a cubic atomic structure, the optical indicatrix is a sphere before stress is applied. The resulting isotropic optical indicatrix is shown in figure 3.9 where $\mathrm{n}_{\varepsilon}$ (the index in the extraordinary or longitudinal direction) is equal to $n_{\omega}$ (the index in the ordinary or lateral direction) by definition. It is key to note that before the silicon experiences any stress:

$$
\mathrm{n}_{\mathrm{o}}=\mathrm{n}_{\mathrm{x}}=\mathrm{n}_{\mathrm{y}}=\mathrm{n}_{\mathrm{z}}
$$

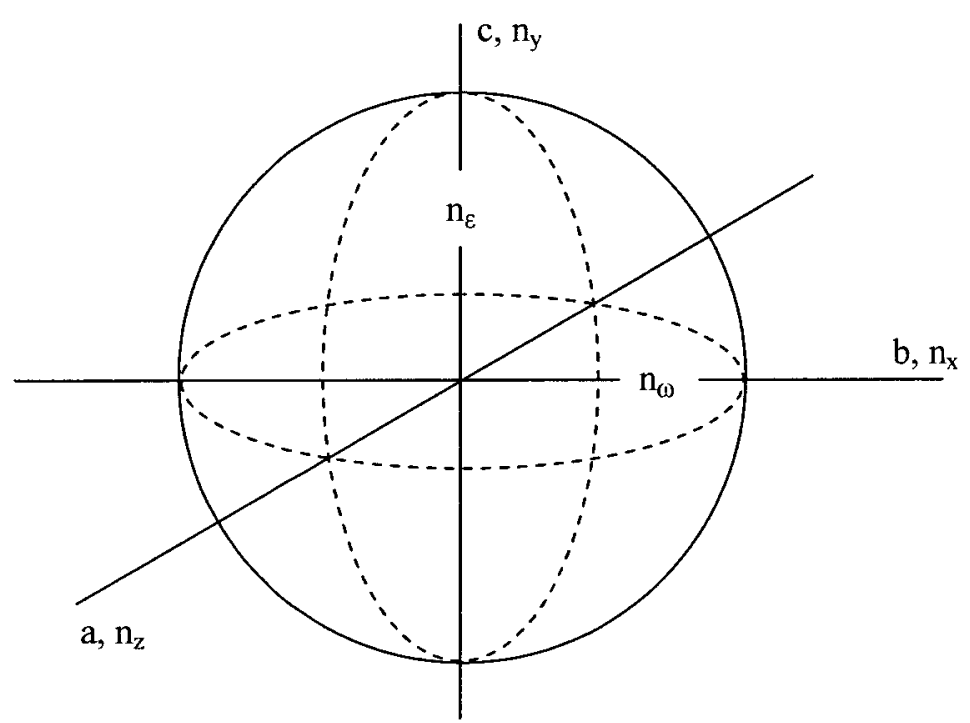

Figure 3.9. Isotropic Optical Indicatrix for a cubic hexoctahedral crystal such as silicon

In general, the indicatrix for silicon is defined by:

$$
\frac{a^{2}}{n_{z}^{2}}+\frac{b^{2}}{n_{x}^{2}}+\frac{c^{2}}{n_{y}^{2}}=1
$$

where
$\mathrm{a}, \mathrm{b}, \mathrm{c}=$ crystallographic axes
$\mathrm{n}_{\mathrm{x}}, \mathrm{n}_{\mathrm{y}}, \mathrm{n}_{\mathrm{z}}=$ principle refractive indices

The relative dielectric impermeability, $\eta$, is defined as: 


$$
\eta=\frac{\varepsilon_{0}}{\varepsilon}=\frac{1}{\varepsilon_{r}}
$$

where $\varepsilon$ is the dielectric permittivity, $\varepsilon_{0}$ is the permittivity of free space, and $\varepsilon_{r}$ is the characteristic dielectric permittivity of the material.

The index of refraction, $n$, is related to $\eta$ by $\varepsilon_{r}$, such that:

$$
n=\sqrt{\varepsilon_{r}}
$$

Therefore:

$$
\eta=\frac{1}{n^{2}}
$$

When a crystal experiences stress, the indicatrix becomes [70]:

$$
\eta_{1} x^{2}+\eta_{2} y^{2}+\eta_{3} z^{2}+2 \eta_{4} y z+2 \eta_{4} z x+2 \eta_{4} x y=1
$$

where

$$
\mathrm{x}, \mathrm{y}, \mathrm{z}=\text { axes of the indicatrix }
$$

The general equation for the photo-elastic effect on the waveguide mode index can be obtained from [71]:

$$
\Delta \eta_{i}=\pi_{i j} \sigma_{j}
$$

where $\boldsymbol{\eta}$ is the dielectric impermeability tensor, $\pi_{i j}$ is a fourth rank stress optic coefficient tensor, and $\sigma$ is the stress tensor.

The change in the index of the silicon when under stress can be solved along the optic axis in the direction the stress is applied via the application of its stress-optic coefficients. To solve for the change of refractive index in silicon for a long slender beam in the $\langle 110\rangle$ crystallographic direction, equation 3.28 is expanded to matrix form in equation 3.29 . 


$$
\left[\begin{array}{c}
\Delta \eta_{1} \\
\Delta \eta_{2} \\
\Delta \eta_{3} \\
\Delta \eta_{4} \\
\Delta \eta_{5} \\
\Delta \eta_{6}
\end{array}\right]=\left[\begin{array}{cccccc}
\pi_{11} & \pi_{12} & \pi_{12} & 0 & 0 & 0 \\
\pi_{12} & \pi_{11} & \pi_{12} & 0 & 0 & 0 \\
\pi_{12} & \pi_{12} & \pi_{11} & 0 & 0 & 0 \\
0 & 0 & 0 & \pi_{44} & 0 & 0 \\
0 & 0 & 0 & 0 & \pi_{44} & 0 \\
0 & 0 & 0 & 0 & 0 & \pi_{44}
\end{array}\right]\left[\begin{array}{c}
\sigma_{x} \\
\sigma_{y} \\
0 \\
0 \\
0 \\
0
\end{array}\right]
$$

Using equation 3.26 and solving for one principle axis, the dielectric impermeability, $\eta$, can be rewritten as:

$$
\eta_{1}=\frac{1}{n_{x}^{2}}
$$

Next consider the change in impermeability as the index changes:

$$
\Delta \eta_{1}=-\left(\frac{2}{n_{x}^{3}}\right) \Delta n_{x}
$$

Due to the cubic nature of the crystal, $n_{0}$ can be taken as an approximation of $n_{x}$ so that equation 3.31 can be rewritten as:

$$
\Delta n_{x}=-\frac{1}{2} n_{0}^{3} \Delta \eta_{1}
$$

Substituting from equation 3.28 gives:

$$
\Delta n_{x}=-\frac{1}{2}\left(n_{0}\right)^{3}\left(\sigma_{x} \pi_{11}\right)
$$

Similarly:

$$
\begin{aligned}
& \Delta n_{y}=-\frac{1}{2}\left(n_{0}\right)^{3}\left(\sigma_{x} \pi_{12}\right) \\
& \Delta n_{z}=-\frac{1}{2}\left(n_{0}\right)^{3}\left(\sigma_{x} \pi_{12}\right)
\end{aligned}
$$

As discussed in section 3.5 , the crystallographic orientation of a SEMI standard (100) silicon wafer is such that the stress on a waveguide bridge aligned to the (110) 
wafer flat will influence the index in the $\mathrm{x}$ and $\mathrm{z}$ indicatrix directions. The axial stress that the waveguide will experience prior to buckling will be the resultant of $\sigma_{x}$ and $\sigma_{y}$ stresses. By resolving the stresses in the $\langle 110\rangle$ direction, the photo-elastic effect on the index becomes $[70,72]$ :

$$
\Delta n_{\langle 110\rangle}=-\frac{1}{2}\left(n_{0}\right)^{3}\left[\sigma_{\langle 10\rangle}\left(\pi_{11}-\pi_{12}\right)\right]
$$

The stress-optic coefficient, $\pi_{11}-\pi_{12}$, for silicon is $-14.4 \times 10^{-13} \mathrm{~Pa}^{-1}$ [73] and the compressive stress, $\sigma_{\{110\rangle}$, is negative; therefore $\Delta n_{\{110\rangle}$ is negative. This will have an important implication for the amount of shift in the Bragg filter wavelength when the waveguide bridge buckles.

\subsection{Thermo-mechanical axial beam deformation}

The fixed-fixed beam, shown in figure 3.10 , is considered to be a "long column" such that the suspended waveguide bridge it represents is much longer than it is wide or thick. For simplicity, the $x$ axis is rotated into alignment with the axis of the beam. The stress along its axis, $\sigma_{x}$, dominates and $\sigma_{y}$ and $\sigma_{z}$ are negligible. For analysis of this structure, $\sigma_{m e c h a n i c a l} \equiv \sigma_{x}$, and the beam is uniformly heated.

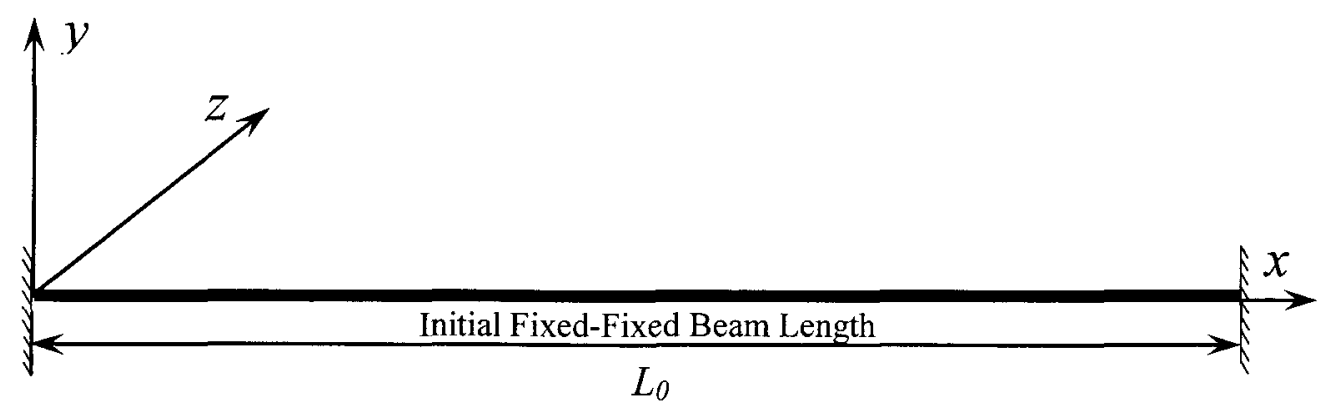

Figure 3.10. Long slender fixed-fixed beam subject to a uniform temperature increase.

Beams whose ends are restrained against translation produce opposing 
mechanical strains to thermal expansion strains and therefore compressive stresses. The total axial strain, $\varepsilon_{t o t a l}$, is the sum of the strain, $\varepsilon_{\text {mechanical }}$, due to the axial stress, $\sigma_{\text {mechanical }}$; and the thermal strain, $\mathcal{E}_{\text {thermal, }}$, due to heating. Therefore the strain created by the waveguide bridge thermally expanding against its fixed supports will have a mechanical component and a thermal component. The total strain for the waveguide bridge in the pre-buckle configuration can be written as:

$$
\varepsilon_{\text {total }}=\varepsilon_{\text {mechanical }}+\varepsilon_{\text {thermal }}
$$

This equation is called the stress-strain-temperature equation for axial deformation of a slender, linear elastic member [74] where $\varepsilon_{\text {mechanical }}$ is the value calculated from equation 3.19 and $\varepsilon_{\text {thermal }}$ is the value calculated from equation 3.18. In the pre-buckle temperature range, the thermal strain in the waveguide bridge is fully constrained. Since the beam ends are restrained against expansion or deflection, they produce opposing mechanical strains to counter the thermal expansion. The total axial strain in this range is zero and equation 3.36 can be rewritten as:

$$
\begin{gathered}
\varepsilon_{\text {total }}=\varepsilon_{\text {mechanical }}+\varepsilon_{\text {thermal }}=0 \\
\varepsilon_{\text {mechanical }}=-\varepsilon_{\text {thermal }}
\end{gathered}
$$

The shift of the Bragg wavelength in the pre-buckle regime will be impacted by the thermal influence of the thermo-optic effect and the stress influence of the photoelastic effect. There will be no change in the Bragg pitch during the pre-buckle regime. Combining equation 3.38 with 3.18 and 3.19 results in an expression for the axial stress in the pre-buckle regime:

$$
\sigma_{\text {mechanical }}=-\alpha \Delta T E
$$


When thermal expansion of the fixed-fixed waveguide bridge increases to a characteristic critical pressure and the beam buckles, the strains are free to develop, and the beam will reach a new stable shape in which the vertical deflection, $\delta$, of the waveguide bridge is related to the total strain in the beam [75].

$$
\varepsilon_{\text {total }} \rightarrow \delta
$$

In the post-buckle configuration, the strain due to mechanical deformation is no longer present since the stress due to thermal expansion has been released in the buckle process. Therefore the total strain for the waveguide bridge in the post-buckle configuration can be written as:

$$
\varepsilon_{\text {total }}=\varepsilon_{\text {thermal }}
$$

Furthermore, as the temperature continues to rise in the post-buckle condition, provided there are no external loads, the thermal expansion will continue to be expressed by $\varepsilon_{\text {thermal }}$ alone. Since the post-buckled waveguide bridge is now free to thermally expand out-of-plane, stress will not develop along the axis of the long slender beam and there will no longer be a photo-elastic effect on the Bragg wavelength. This is an important assertion to make when analysing the difference in the thermal sensitivity of the Bragg wavelength in the pre- and post-buckle regimes, as well as the magnitude of the Bragg wavelength shift when the waveguide bridge buckles. This model will be more fully explained in section 4.4 .3 by accounting for the residual, bending, and compressive stress in the beam as it deflects through to its post-buckle condition. The dependency of the Bragg wavelength in the pre-buckle regime is $\lambda_{B}$ (thermo-optic, stress-optic), while the dependency of the Bragg wavelength in the post buckle regime is $\lambda_{B}$ (thermo-optic, thermo-mechanic). 


\subsection{Beam Buckle Theory}

With an understanding of the pre- and post-buckle thermo-mechanical axial beam deformation, it is now time to determine the shape that this deformation will force the fixed-fixed beam into when it buckles. The bridge waveguide structure that contains the Bragg filter is usually referred to as a beam, column or rod; while the anchored configuration is variably described as a built-in beam, a clamped-clamped beam, or a fixed-fixed beam.

The fixed-fixed beam that forms the suspended waveguide bridge in this thesis is assumed to be rigid, straight, and fixed at both ends; and meet the conditions of a "long column" discussed in the previous section. A long column will allow the application of the Euler-Bernoulli beam displacement theory, while a fixed-fixed configuration will dictate the amount of thermal expansion required to develop critical buckling force, as well as the height of deflection that occurs when the bridge buckles.

Figure 3.11 labels the important elements of the beam that will be used to describe its buckle behaviour. The length of the beam at its rest state, or pre-buckle configuration, is $L_{0}$. As the silicon beam is heated, thermal expansion occurs. If the beam were free to expand along the $\mathrm{x}$ axis, this thermal expansion would be represented by $d x$. Since the bridge is fixed, however, and not free to expand axially, $d x$ results in axial force, $P$, with a bending moment, $M$.

As the fixed-fixed beam continues to expand, the force will continue to increase until the bridge buckles. This critical buckling force, $P_{c r}$, is known as the Euler buckling load. The critical load as a result of the axial stress of thermal expansion is defined as: 


$$
P_{c r}=\sigma_{c r} A
$$

where the formula for calculating the critical stress, $\sigma_{c r}$, is described by equation 3.22 .

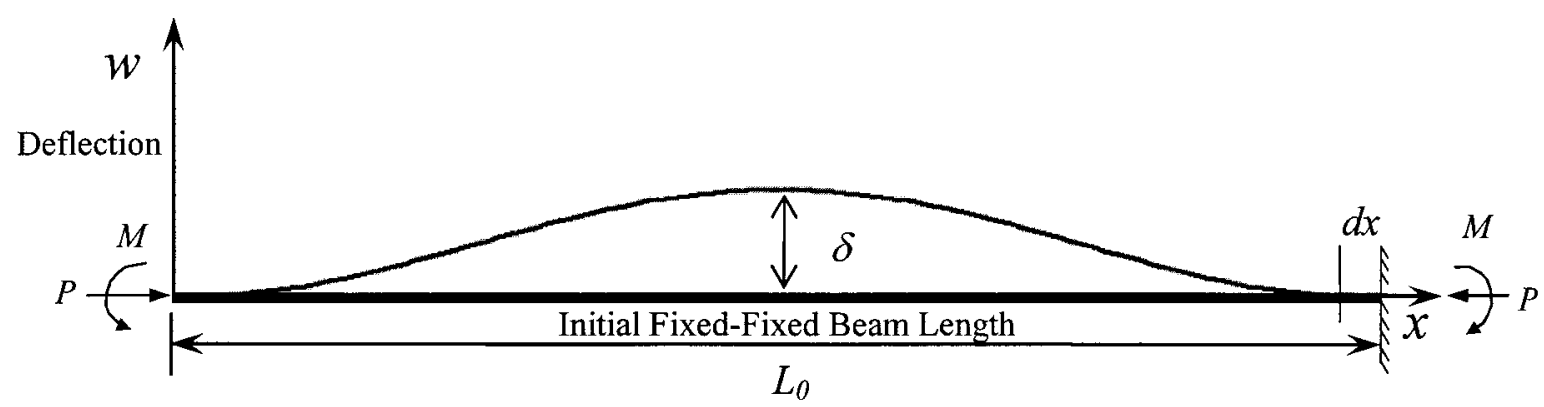

Figure 3.11. The buckling mode shape of a fixed-fixed beam where the $w$-axis is exaggerated to show the vertical displacement, $\delta$, of the beam.

If the beam is of sufficient slenderness it will form a shape called the mode shape, or buckling mode. This is the shape depicted in figure 3.11 , in which the $w$-axis is exaggerated to show the vertical displacement of the beam. The distance that the centre of the beam will deflect out-of-plane in its first buckling mode is $\delta$.

Although a straight beam could theoretically buckle in the second buckling mode, this would only physically happen if the beam were laterally constrained at its centre to prevent it from buckling in the first mode [74]. Higher order mode buckling has been achieved in MEMS beams that were not initially straight [76,77]. Such structures exhibit bi-stability in that they are stable in two different mode shapes at room temperature.

The governing equation that describes the relationship between the waveguide bridge deflection, $w(x)$, and the load, $P$, due to the axial thermal expansion, is the EulerBernoulli beam equation [74]:

$$
E I \frac{d^{4} w}{d x^{4}}+P \frac{d^{2} w}{d x^{2}}=0
$$

where $E I$ is the flexural rigidity (comprised of modulus of elasticity, $E$ and moment of 
inertia, $I$ ), and the function $w(x)$ describes the deflection at a position $x$ along the beam. Due to the fixed-fixed configuration of the beam, the boundary conditions are $w(0)=w^{\prime}(0)=w(L)=w^{\prime}(L)=0$.

M. Vangbo developed an analytical model to determine the lateral displacement of a long fixed-fixed beam [78]. Considering the buckling mode shape that the solution of $w(x)$ will take, equation 3.43 can be solved as an eigenvalue problem. Setting $w=e^{\lambda x}$, equation 3.43 can be rewritten as

$$
\lambda^{4}+\frac{P}{E I} \lambda^{2}=0
$$

where

$$
\lambda= \pm \sqrt{\frac{P}{E I}} i
$$

The general homogeneous solution for 3.43 becomes

$$
w=A \sin \sqrt{\frac{P}{E I}} x+B \cos \sqrt{\frac{P}{E I} x}+C+D x
$$

where $\mathrm{A}, \mathrm{B}, \mathrm{C}$ and $\mathrm{D}$ are constants that are determined by using the previously defined boundary conditions. If equation 3.43 is solved for the condition at which the load due to thermal expansion, $P$, is 0 , the beam has buckled and $P$ becomes $P_{c r}$ [79]. The critical axial compressive load that will cause a fixed-fixed beam to buckle is given by:

$$
P_{c r}=\frac{4 \pi^{2} E I}{L^{2}}
$$

with its associated buckle shape function:

$$
w(x)=\frac{\delta}{2}\left(1-\cos \frac{2 \pi x}{L_{0}}\right)
$$

This function describes the buckling mode shape plotted in figure 3.11 [80]. The 
critical buckling load, $P_{c r}$, is the eigenvalue, and the buckling mode shape, $w(x)$, is its eigenfunction. The value of the maximum deflection is $\delta$. Because the beam is statically indeterminate, the value of $\delta$ cannot be derived from equation 3.43.

To complete the mechanical description of this beam, its moment of inertia and radius of gyration must be defined. The moment of inertia for a rectangular beam is:

$$
I=\frac{1}{12} b d^{3}
$$

where $b$ is the beam width, and $d$ is the beam thickness. This type of cross-section can also be called "prismatic".

The cross-section that describes the rib waveguide bridge of this thesis is commonly called a "T beam". Figure 3.12 depicts the geometry to describe the calculation of the centroid and moment of inertia for this shape.

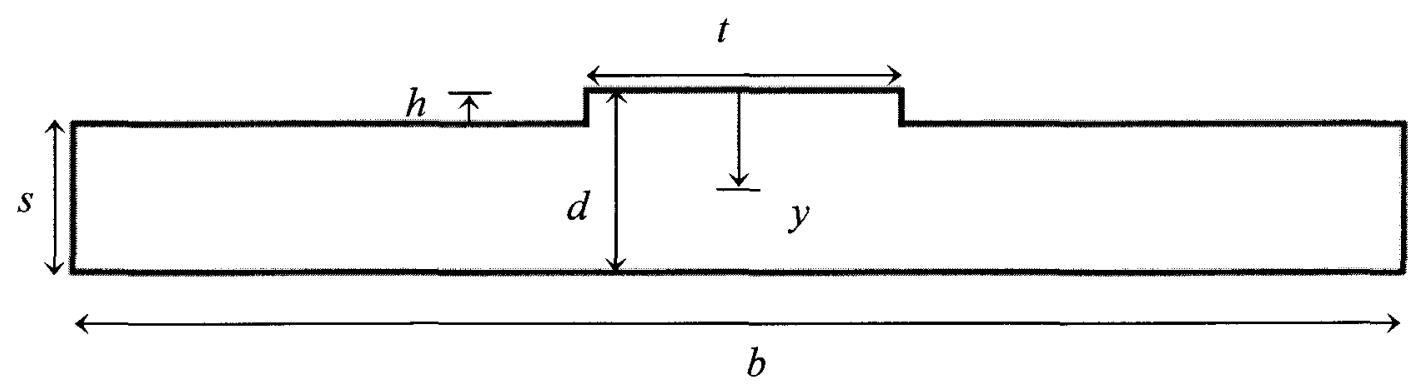

Figure 3.12. Moment of Inertia diagram of rib waveguide cross-section

The centroid, $y$, is [74]:

$$
y=d-\frac{d^{2} t+s^{2}(b-t)}{2(b s+h t)}
$$

The moment of inertia, $I$, is:

$$
I=\frac{1}{3}\left[t y^{3}+b(d-y)^{3}-(b-t)(d-y-s)^{3}\right]
$$


Additionally, the radius of gyration, $r$, is:

$$
r=\sqrt{\frac{I}{A}}
$$

where $A$ is the cross-sectional area of the bridge.

With $I$ and $r$ defined, we can now obtain the Euler condition of the waveguide bridge. The Euler critical buckling load, $P_{c r}$, and the buckling mode, $w(x)$, is valid for long columns for which the slenderness ratio leads to a critical stress below the yield stress of the beam material [74]. The slenderness ratio is a ratio of the beam length to the radius of gyration. In the case of polycrystalline silicon, the Euler buckling formula is considered valid for a slenderness ratio greater than 26 [81]. Since the modulus of elasticity and yield strength is similar to single crystal silicon, the same slenderness ratio can be used. The slenderness ratio of the $500 \mu \mathrm{m}$ to $4000 \mu \mathrm{m}$ structures developed for this thesis range from 400 to 3000 , easily complying.

An important realisation is made from the nature of equation 3.48. In determining the buckling mode shape, and ultimately its buckle height, the width of the beam does not mater, nor does its cross-sectional geometry. There is also no material component in this equation. This function describes the mathematical mode shape that a long fixed-fixed buckled beam will conform to in its minimum energy state. The material properties of the beam and its moment of inertia are not a consideration.

Another important insight is the distinction between the axial thermal expansion, $d x$, and the beam expansion related to the buckle height, $\delta$, caused by $d x$. The buckle expansion is related to the non-mechanical buckling mode shape of the beam, while the magnitude of thermal expansion is governed by the laws of mechanics as described by the beam's material properties and dimensions. The relationship between the two is that 
for a comparatively small thermal expansion, $d x$, a large $\delta$ is generated. This is useful for MEMS buckle beam actuator designs, where a comparatively small in-plane actuation can cause larger out-of-plane travel in a second actuator $[17,82,83]$. Depending on the location along the beam, a greater grating pitch expansion, $\Delta \Lambda$, also occurs; as much as double the value of $d x$. A quantitative treatment of this is found in section 4.4.2.

Recalling the beam profile described in figure 3.11, the relationship between $d x$ and $\delta$ for a long fixed-fixed buckled beam is [84]:

$$
\delta=\sqrt{\frac{d x 4 L_{0}}{\pi^{2}}}
$$

With $\delta$ determined, it is now possible to give the buckling mode shape a quantitative amplitude.

The mid-span deflection equation 3.53 can also be approximated for a long fixedfixed beam by the following relationship [75]:

$$
\delta=\frac{2 L_{0}}{\pi} \sqrt{\varepsilon_{T}+\frac{\varepsilon_{T}^{2}}{2}}
$$

where $\varepsilon_{T}$ is the thermal strain. Once the critical buckling temperature that results in $P_{c r}$ has been calculated, comparing the result of this relationship for various beam lengths against 3.53 will help confirm the validity of the beam deflection height analysis.

The final work of this section is to use line segment theory to determine the change in Bragg grating length (or pitch) on the bridge in which it resides. To begin, one must recall that the length of the curve along any two close points $P_{1}$ and $P_{2}$ can be calculated by using the line segment formula [85]:

$$
\left|P_{1} P_{2}\right|=\sqrt{\left(x_{2}-x_{1}\right)^{2}+\left(y_{2}-y_{1}\right)^{2}}
$$


Now consider the length of the beam as a polygon. The length, $L$, of the polygon can be computed by adding the lengths of the line segments that form the polygon.

$$
L=\lim _{\|P\| \rightarrow 0} \sum_{i=1}^{n}\left|P_{i-1} P_{i}\right|
$$

An integral formula for the new length of the buckled beam, $L$, can be derived in the case where the buckle function $w(x)$ has a continuous derivative on any two points on the beam, $[a, b]$ such that:

$$
L=L_{0}+\Delta L=\int_{a}^{b} \sqrt{1+\left[w^{\prime}(x)\right]^{2}} d x
$$

where $a$ and $b$ are defined over the region of the waveguide bridge where the Bragg grating filter has been integrated, $L_{0}$ is the initial length of the entire grating and $\Delta L$ corresponds to the change in length of the entire grating due to the buckle shape of the waveguide bridge. The change in the Bragg pitch, $\Delta \Lambda$, can be determined from dividing $\Delta L$ by the number of periods in the grating.

The derivative of equation 3.48 is:

$$
w^{\prime}(x)=\delta \frac{\pi}{L_{0}} \sin \left(\frac{2 \pi x}{L_{0}}\right)
$$

and the integral is:

$$
L_{0}+\Delta L=\int_{0}^{L} \sqrt{1+\left[\delta \frac{\pi}{L_{0}} \sin \left(\frac{2 \pi x}{L_{0}}\right)\right]^{2}} d x
$$

This equation reduces to a non-integrable function that cannot be solved analytically. It can either be solved numerically, or approximated via a Taylor series expansion provided $\Delta L$ is small [78]. The approximation of equation 3.59 is: 


$$
L_{0}+\Delta L=\int_{0}^{L}\left\{1+\frac{\left[w^{\prime}(x)\right]^{2}}{2}\right\} d x
$$

\subsection{Thermal Tuning}

The previous sections outlined the effect that thermal tuning will have on the thermooptic, thermo-mechanic, photo-elastic and buckling mechanics of the device that is described in this thesis. Section 3.7 explained the balance between thermal strain and compressive stress in the pre-buckle regime, the release of stress in the buckle regime, and the thermal strain active in the post-buckle regime. Section 3.8 outlined the buckling mode Bragg grating expansion in the buckle regime. The different combinations of material and structural effects will result in different tuning responses in each regime. By collecting the different effect combinations, a qualitative model of the thermal influence on the Bragg filter wavelength over the three regimes of operation will be developed and presented at the end of this section.

The device layer that the bridge waveguide is anchored to will be fixed at a reference temperature such that $T_{\infty}=$ room temperature. The beam itself is surrounded by air with a $2 \mu \mathrm{m}$ gap from the bridge to the substrate. External heating, when used, will occur along the middle section of the beam. Thus the entire waveguide bridge will not be held at a single elevated temperature. Since the Bragg grating filter is itself integrated along the middle section of the bridge, however, assuming a uniform axial thermal profile on the effective index of the grating is likely a good approximation. During the experimental characterisation procedure, the waveguide bridge temperature will be increased in $1.0^{\circ} \mathrm{C}$ increments with a 60 second pause for a steady state to develop and 
the optical measurement to be taken.

The two primary critical buckle effects on the thermo-mechanical behaviour of the waveguide bridge include constrained axial thermal expansion, and a surface distribution of temperature which leads to compression on the heated side of the bridge. Due to the small thermal increments between each steady state, it is likely the influence of vertical non-uniformities in the thermal profile will have a negligible influence on the critical buckle temperature compared to the cumulative constrained thermal expansion along the axis of the bridge.

In the pre-buckle regime of operation, starting at room temperature, there is no thermal actuation or waveguide bridge deflection. The tunability of the Bragg filter wavelength is a combination of the thermo-optic effect due to temperature shift and the photo-elastic effect due to stress in the waveguide from constrained thermal expansion. The thermal sensitivity of the Bragg wavelength in the pre-buckle region of operation will be the sum of these effects on the effective index of the Bragg grating within the waveguide. Since the waveguide bridge is fixed at both ends, axial strain will not be present in the pre-buckle regime and therefore the Bragg grating pitch will not change.

The buckle regime occurs as the waveguide bridge surpasses its critical buckling pressure and the device transitions from the pre- to post-buckle regimes. When buckling, the bridge deflects through a region of instability until it reaches its stable buckling mode shape. In doing so, the compressive stress that has built-up in the beam is released. This removes the cumulative influence of the photo-elastic effect on the effective index of the Bragg filter waveguide. The discrete shift of the Bragg filter wavelength is increased further by the expansion of the Bragg grating pitch as a result of the buckling mode shape 
of the rib waveguide.

The condition of the beam at the beginning of the post-buckle regime is the result of the buckling mode shape. The period of the Bragg filter is now free to expand as the temperature continues to increase over this range of thermal operation. The thermo-optic effect will also continue its influence on the shift in Bragg wavelength. Since the stress has been released and will not return appreciably as the beam continues to deflect out-ofplane [75], there will be no stress effect on the index of the waveguide filter in the postbuckle regime. Since the negative photo-elastic influence on the effective index is no longer present and the Bragg pitch is now free to expand, the rate of change in the Bragg wavelength as a result of change in temperature should be greater in the post-buckle regime than in the pre-buckle regime.

When decreasing the temperature from the post-buckle regime, it has been shown that the return to the pre-buckle state occurs at a lower temperature than the original buckle temperature [86]. This hysteresis or bi-stable effect has been analysed and characterised with thermally actuated silicon and polysilicon fixed-fixed bridges as well as from a general mechanics frame of analysis [78]. Although this effect is not a primary area of investigation in this thesis, it is an interesting phenomena to acknowledge nonetheless. Based on the predicted behaviour discussed thus far, figure 3.13 presents a qualitative plot of the critical thermal states for a suspended waveguide Bragg filter and the corresponding shifts in the Bragg filter wavelength, while table 3 describes them. 


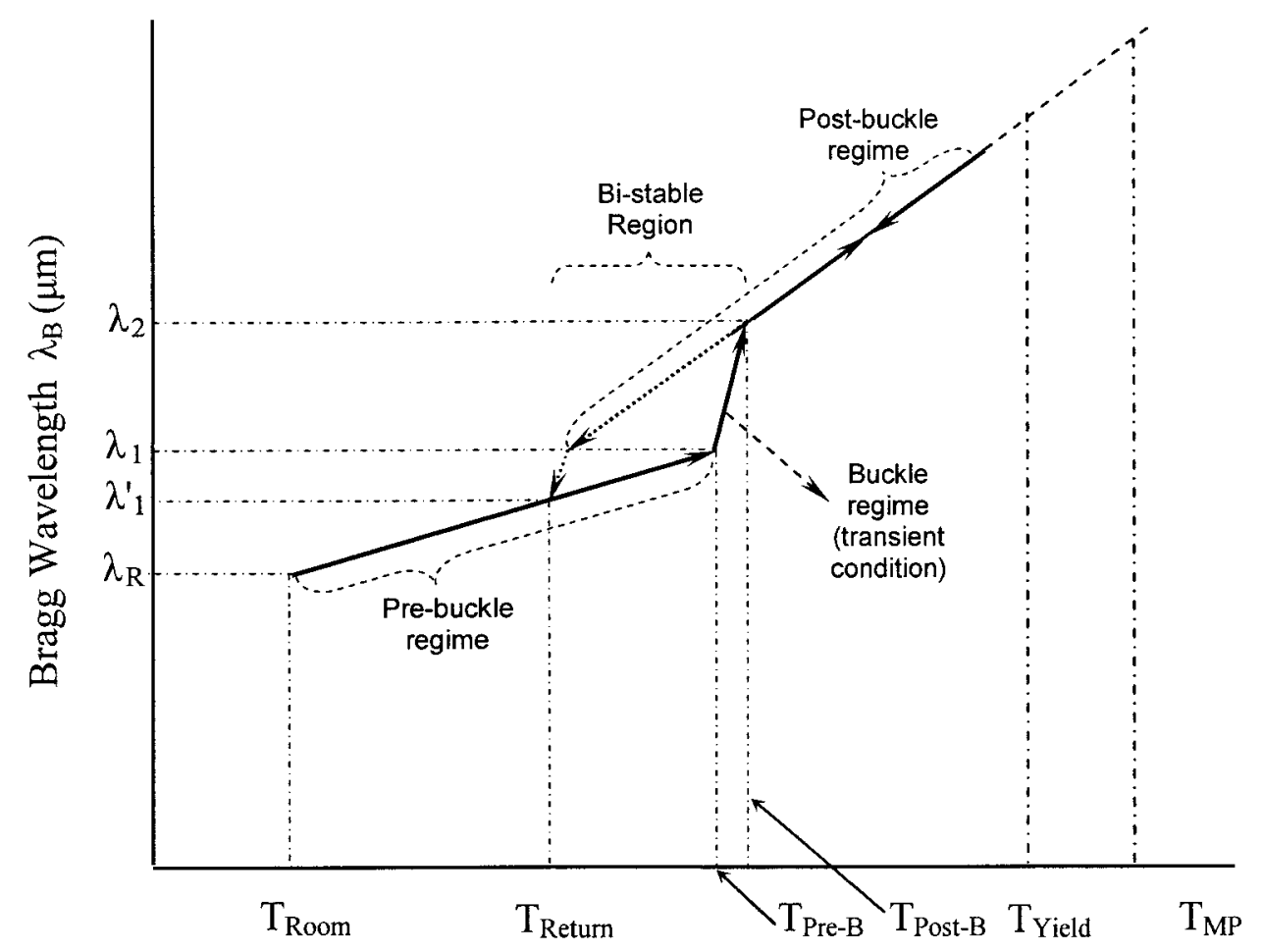

Waveguide Bridge Temperature $\left({ }^{\circ} \mathrm{C}\right)$

Figure 3.13: Important states for the waveguide bridge filter over pre-buckle to postbuckle regimes, showing the Bragg wavelength versus the waveguide bridge temperature [after 86].

\begin{tabular}{|c|l|}
\hline Device states & \multicolumn{1}{c|}{ Description } \\
\hline$\lambda_{\mathrm{R}}$ versus $\mathrm{T}_{\mathrm{Room}}$ & $\begin{array}{l}\text { The Bragg wavelength of the waveguide } \\
\text { filter at room temperature. }\end{array}$ \\
\hline$\lambda_{1}$ versus $\mathrm{T}_{\text {Pre-B }}$ & $\begin{array}{l}\text { The Bragg wavelength at the highest pre- } \\
\text { buckle temperature of the waveguide } \\
\text { filter. }\end{array}$ \\
\hline$\lambda_{2}$ versus $\mathrm{T}_{\text {Post-B }}$ & $\begin{array}{l}\text { The Bragg wavelength at the lowest post- } \\
\text { buckle temperature of the waveguide. }\end{array}$ \\
\hline$\lambda_{1}^{\prime}$ versus $\mathrm{T}_{\text {Return }}$ & $\begin{array}{l}\text { The Bragg wavelength at the temperature } \\
\text { when the waveguide returns to its pre- } \\
\text { buckle configuration. }\end{array}$ \\
\hline $\mathrm{T}_{\text {Yield }}$ & $\begin{array}{l}\text { The thermal actuation temperature at } \\
\text { which the crystal structure of the silicon } \\
\text { breaks down such that the bridge can not } \\
\text { elastically return to } \lambda_{1}^{\prime} .\end{array}$ \\
\hline $\mathrm{T}_{\mathrm{MP}}$ & $\begin{array}{l}\text { The catastrophic actuation temperature at } \\
\text { which the silicon reaches melting point. }\end{array}$ \\
\hline
\end{tabular}

Table 3. List of critical waveguide bridge states that correspond to Fig. 3.13, with descriptions. 


\section{Analysis and Design}

Theory introduced in the previous chapter will be used to analyse the tuning effects for the device proposed in this thesis. The analysis will be divided into material and structural elements that, when combined, will predict and describe the behaviour of the suspended waveguide filter over specific regions of thermal operation. This analytical model will then be used to design the device.

The structural elements include a single mode waveguide, a Bragg grating, an integrated heater, and a waveguide bridge. The material elements within the waveguide bridge include the thermo-optic, photo-elastic, and thermo-mechanic effects on the effective index and pitch of the integrated Bragg grating filter, over the pre- to postbuckle regions of thermal tuning.

It is not uncommon for the flow between design, fabrication, and characterisation to be an iterative process in a MEMS or MOEMS based device. This is expected for a device in which multiple structural and material elements are coupled to each other. Modelling the coupled behaviour of the design with numeric analysis can be a significant undertaking on its own and as such an analytic model is often a good "first best guess" to predict the fundamental operating characteristics of the device. Numeric analysis can then optimize the design parameters. This approach is an effective way to isolate or discover useful or unique performance characteristics in a new device.

\subsection{Waveguide}

Analysis of the geometry required to support the single mode condition in an SOI rib waveguide has been conducted by Soref et al. [55]. Given an SOI rib waveguide with the 
dimensions labelled in figure 4.1, Soref used mode-matching and beam-propagation methods to develop equation 4.1 , that defines the dimensions required for single mode operation:

$$
\frac{H}{w}<\gamma+\frac{\frac{h}{H}}{\sqrt{1-\left(\frac{h}{H}\right)^{2}}}
$$

where $H$ is the device layer thickness of the SOI, $h$ is the slab height, $w$ is the rib width and $\gamma$ is a design factor. The difference between $H$ and $h$ is the rib waveguide height. For single mode propagation, the conditions $h / H>0.5$ and $y=0.3$ must hold.

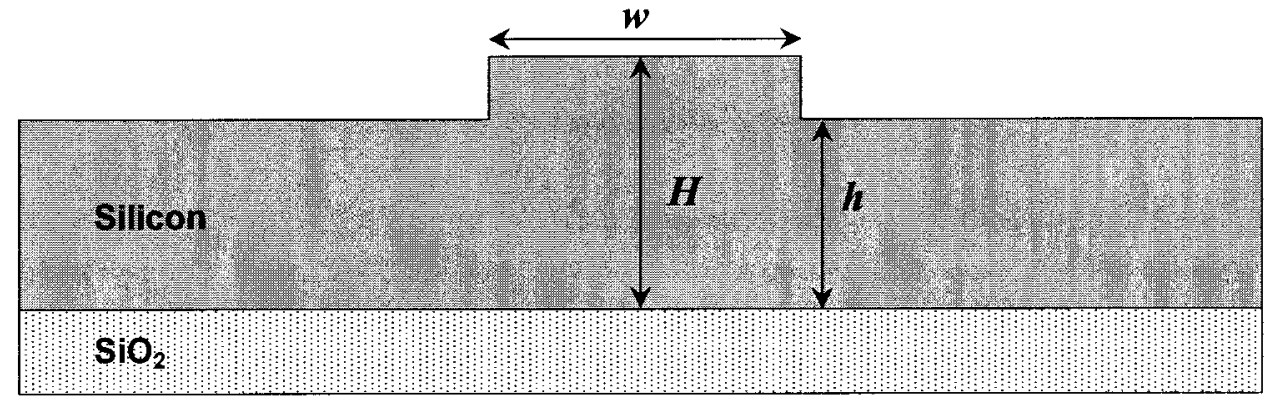

Figure 4.1. Cross-section of an SOI rib waveguide with principal dimensions marked. The handle layer of silicon is not shown in this figure.

Soref's analysis demonstrates that all the higher order modes in the central rib region are filtered out by leaking laterally along the slab portion of the guide, adjacent to the rib. At a distance within $2.0 \mathrm{~mm}$ along the guide, the field intensity would be close to the fundamental mode of the waveguide [55]. As the light propagates along the waveguide, all the higher order modes in the central rib region leak away, leaving only the fundamental mode.

Pogossian et al, used the effective index method outlined in section 3.1.4, and set $\gamma$ to 0 . Using the effective index method he derived the same relationship [87]. 
Pogossian then compared his theory to experimental results from Rickman et al. [88]. A plot of the ratios $w / H$ versus $h / H$, that delineates single mode from multimode operation, is shown in figure 4.2. This plot combines the work of Soref, Pogossian and Rickman. Pogossian's function was used to resolve the rib dimensions for the device designed in this thesis to ensure the waveguide only supported a single mode.

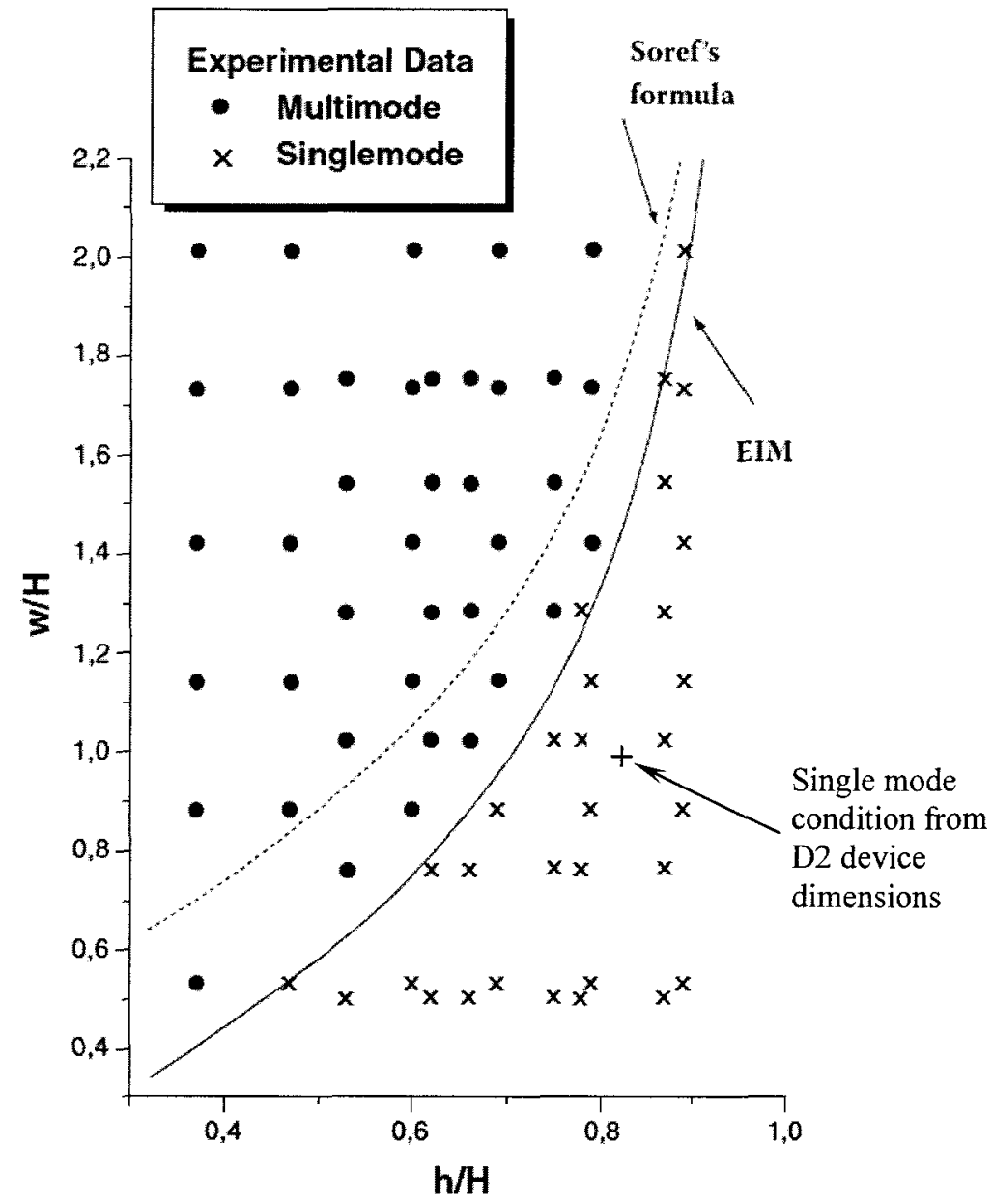

Figure 4.2. $\mathrm{W} / \mathrm{H}$ as a function of $\mathrm{h} / \mathrm{H}$ for the single mode propagation limit [87]

Choosing the device layer thickness of the SOI begins the design process. By using equation 4.1 and the multi/single mode delineation line of figure 4.2 , the thickness, $H$, will govern the rib width and height to ensure single mode operation. To physically realise these dimensions, the rib width will be a product of the waveguide mask linewidth 
and the rib height will be determined by the etch process.

The first iteration of the design-fabrication-characterisation flow made use of SOI with a $2.5 \mu \mathrm{m}$ device layer thickness and a BOX thickness of $1.0 \mu \mathrm{m}$. In order to increase the length of a reliable waveguide bridge release to the range of $2000 \mu \mathrm{m}$ to $4000 \mu \mathrm{m}$, the thickness was increased to $5.0 \mu \mathrm{m}$ with a BOX thickness of $2.0 \mu \mathrm{m}$. It is this SOI profile that will be used throughout the chapter to develop the design for the device. The rib waveguide geometry includes a device layer thickness, $H=5.0 \mu \mathrm{m}$; a rib width, $W=5.0 \mu \mathrm{m}$; and a rib height, $h=0.89 \mu \mathrm{m}$. Using Pogossian's mode propagation limit chart with these dimensions confirms the single mode condition of this waveguide.

To verify that the established waveguide geometry would support the single mode condition, the Apollo Photonics Optical Waveguide Mode Solver (OWMS) [89] was used to calculate the fundamental transverse electric (TE) mode of the suspended rib waveguide. While Pogossian's chart was derived for the general case of an SOI rib waveguide, Apollo would analyse the specific design, including the overall waveguide bridge width of 30 microns and the 2 micron air gap to the handle silicon. The index of silicon, intrinsic to the Pogossian chart, was also required to complete the computation. The index of silicon at room temperature for a wavelength of $1550 \mathrm{~nm}$ is 3.481 [66].

Apollo was used to calculate the mode profile using an effective index method mode solver. The cross-section of the rib waveguide mode profile is shown in figure 4.3 in which the single mode does not leak away in the slab as the higher modes do. 


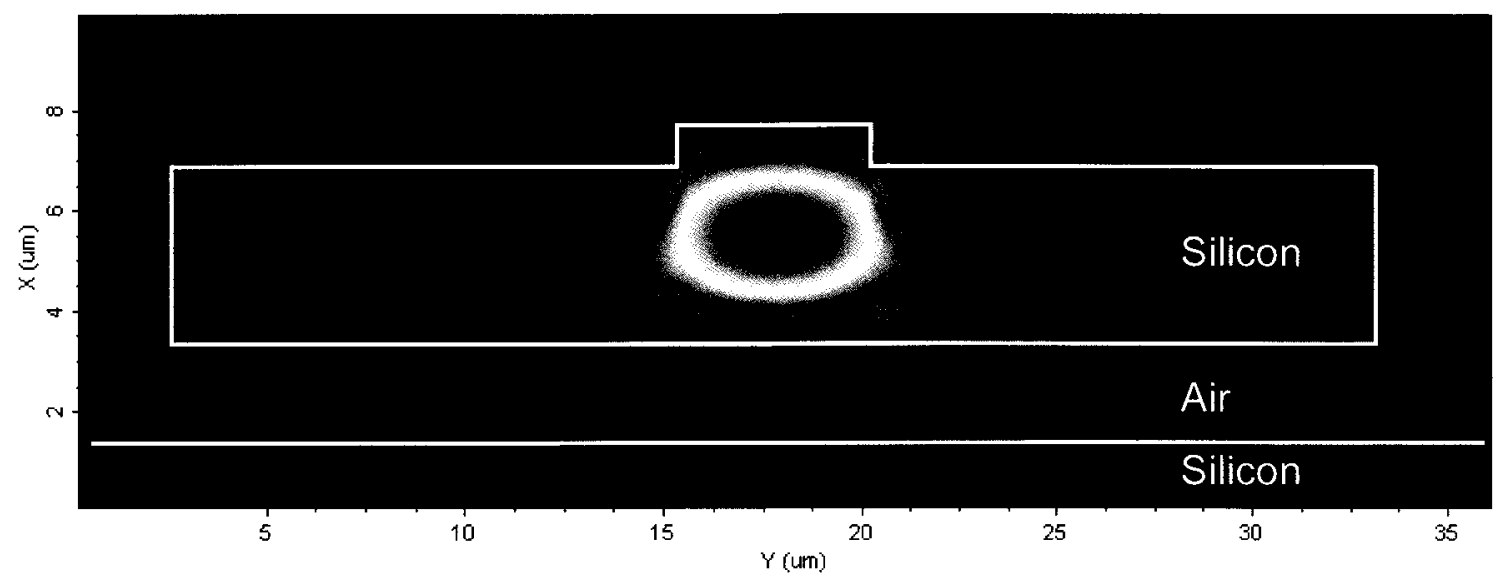

Figure 4.3: Single mode profile of suspended rib waveguide with $H=5.0 \mu \mathrm{m}, w=5.0$ $\mu \mathrm{m}$, and $h=890 \mathrm{~nm}$.

\subsection{Bragg Grating}

The numeric analysis provided by Apollo was a useful tool to compute the core index, $n_{\text {core, }}$ and propagation constant, $\beta$, along the rib waveguide axis over a range of wavelengths. Analysis of the Bragg wavelength, $\lambda_{B}$, reflected by the Bragg grating filter is achieved using the Bragg equation 3.5.

$$
\lambda_{B}=2 n_{e f f} \Lambda
$$

The value $n_{\text {core }}=3.477$ from Apollo is an approximation of $n_{e f f}$, and $\Lambda=225 \mathrm{~nm}$ produces a $\lambda_{B}$ of $1564.7 \mathrm{~nm}$. This analytic result is the iterative starting point for the material and structural design of the device in this thesis. When equation 3.5 is expanded to take into account the thermal and mechanical effects on the performance of the device described in chapter 3 , the shift in $\lambda_{B}$ as the temperature changes, $\Delta \lambda_{B} / \Delta T$, can be calculated.

The coupled mode reflectance equation developed in chapter 3 can compute the Bragg reflectance response over any range of wavelengths. The propagation constant data was computed by Apollo over a spectral range and read into a coupled mode 
reflection program that calculated the coupling coefficient and reflectance over the spectral response using the equations introduced in chapter 3.

$$
\begin{gathered}
\kappa=\frac{2 \pi^{2}}{3 l \lambda} \frac{n_{\text {core }}^{2}-n_{\text {air }}^{2}}{n_{\text {core }}}\left(\frac{a}{t_{g}}\right)^{3}\left[1+\frac{3(\lambda / a)}{2 \pi\left(n_{\text {core }}^{2}-n_{\text {air }}^{2}\right)^{1 / 2}}+\frac{3(\lambda / a)^{2}}{4 \pi^{2}\left(n_{\text {core }}^{2}-n_{\text {air }}^{2}\right)}\right] \\
A(z) e^{i \beta z}=B(0) \frac{i \kappa \exp \left(i \beta_{0} z\right)}{-\Delta \beta \sinh \left(S L_{B}\right)+i S \cosh \left(S L_{B}\right)} \cdot \sinh \left[S\left(z-L_{B}\right)\right]
\end{gathered}
$$

Apollo was used to generate the effective core index and propagation constant of the rib waveguide for wavelengths ranging from 1500 to $1600 \mathrm{~nm}$ in steps of $10 \mathrm{pm}$. At $1550 \mathrm{~nm}$ the index of bulk silicon is 3.481 . The result at $1550 \mathrm{~nm}$ was $n_{\text {core }}=3.477$ and $\beta=14.0939 \mu \mathrm{m}^{-1}$. Additional inputs to the program were the following structural parameters: $\Lambda, L_{B}, a$, and $t_{g}$. The grating pitch, $\Lambda=225 \mathrm{~nm}$, and the waveguide thickness, $t_{g}=5 \mu \mathrm{m}$. The grating length, $L_{B}$, and the grating depth, $a$, will be varied in the analysis.

The equivalent core index and the array of propagation constants over a range of wavelengths was used to generate the spectral response of a Bragg filter with the given rib waveguide and grating geometry. From this plot such characteristics as the strength of reflectance, $R$, and the stopband at $50 \%$ of peak reflectivity, $\delta \lambda_{3 d B}$, were determined in addition to $\lambda_{B}$. These quantities are labelled in a spectral reflectivity plot in figure 4.4. The Bragg wavelength calculated with equation $3.5, \lambda_{B}=1564.7 \mathrm{~nm}$, is very close to the value taken graphically from the coupled mode theory, $\lambda_{B}=1564.6 \mathrm{~nm}$.

The importance of this analysis is to provide some indication of how the geometry of the grating will effect its reflectivity, bandwidth, and channel isolation. The effect on each can be determined by fixing the grating pitch and waveguide thickness, 
and varying both the length of the grating and the grating depth in turn. The following geometries will be common to each grating design analysis. The waveguide height, $H$, also known as $t_{g}=5 \mu \mathrm{m}$; the rib height $=890 \mathrm{~nm}$, and the grating pitch, $\Lambda=225 \mathrm{~nm}$. The grating depth, $a$, will be set to $100 \mathrm{~nm}, 200 \mathrm{~nm}$ and $300 \mathrm{~nm}$ with a constant Bragg grating length, $L_{B}=1000 \mu \mathrm{m}$. Alternately, $L_{B}$ will be set to $500 \mu \mathrm{m}, 1000 \mu \mathrm{m}$, and $2000 \mu \mathrm{m}$ with a constant $a=200 \mathrm{~nm}$. The resulting plots are shown in figure 4.5 and figure 4.6 , and summarised in table 4 . The product of the coupling coefficient, $\kappa$, and the grating length is a commonly used indicator of the strength of the grating and its bandwidth. The $\kappa L_{B}$ grating strength factor is also included in the summary.

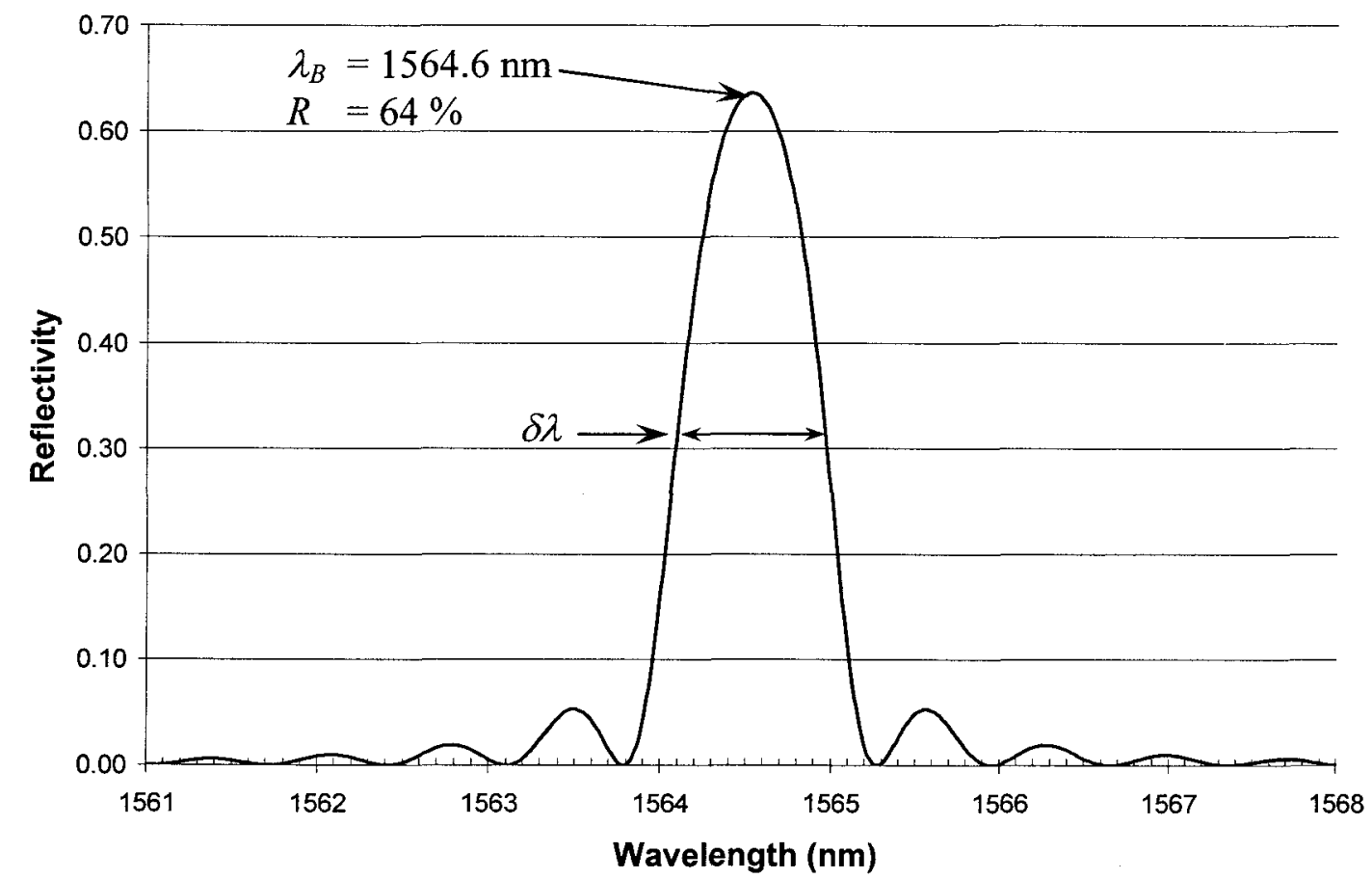

Figure 4.4: Reflectivity of the Bragg filter as a function of wavelength deviation from the Bragg condition with $a=200 \mathrm{~nm}$ and $L_{B}=500 \mu \mathrm{m}$. 


\begin{tabular}{|c|c|c|c|c|c|c|c|}
\hline \multicolumn{4}{|c||}{$L_{B}=1000 \mu \mathrm{m}$} & \multicolumn{5}{c|}{$a=200 \mathrm{~nm}$} \\
\hline$R \%$ & $a(\mathrm{~nm})$ & $\delta \lambda(\mathrm{nm})$ & $\kappa L_{B}$ & $R \%$ & $L_{B}(\mu \mathrm{m})$ & $\delta \lambda(\mathrm{nm})$ & $\kappa L_{B}$ \\
\hline 23.5 & 100 & 0.34 & 0.53 & 63.6 & 500 & 0.86 & 1.1 \\
\hline 95.1 & 200 & 0.66 & 2.2 & 95.1 & 1000 & 0.66 & 2.2 \\
\hline 100 & 300 & 1.54 & 5.6 & 100 & 2000 & 0.56 & 4.4 \\
\hline
\end{tabular}

Table 4. Summary of grating analysis based on various grating dimension combinations.

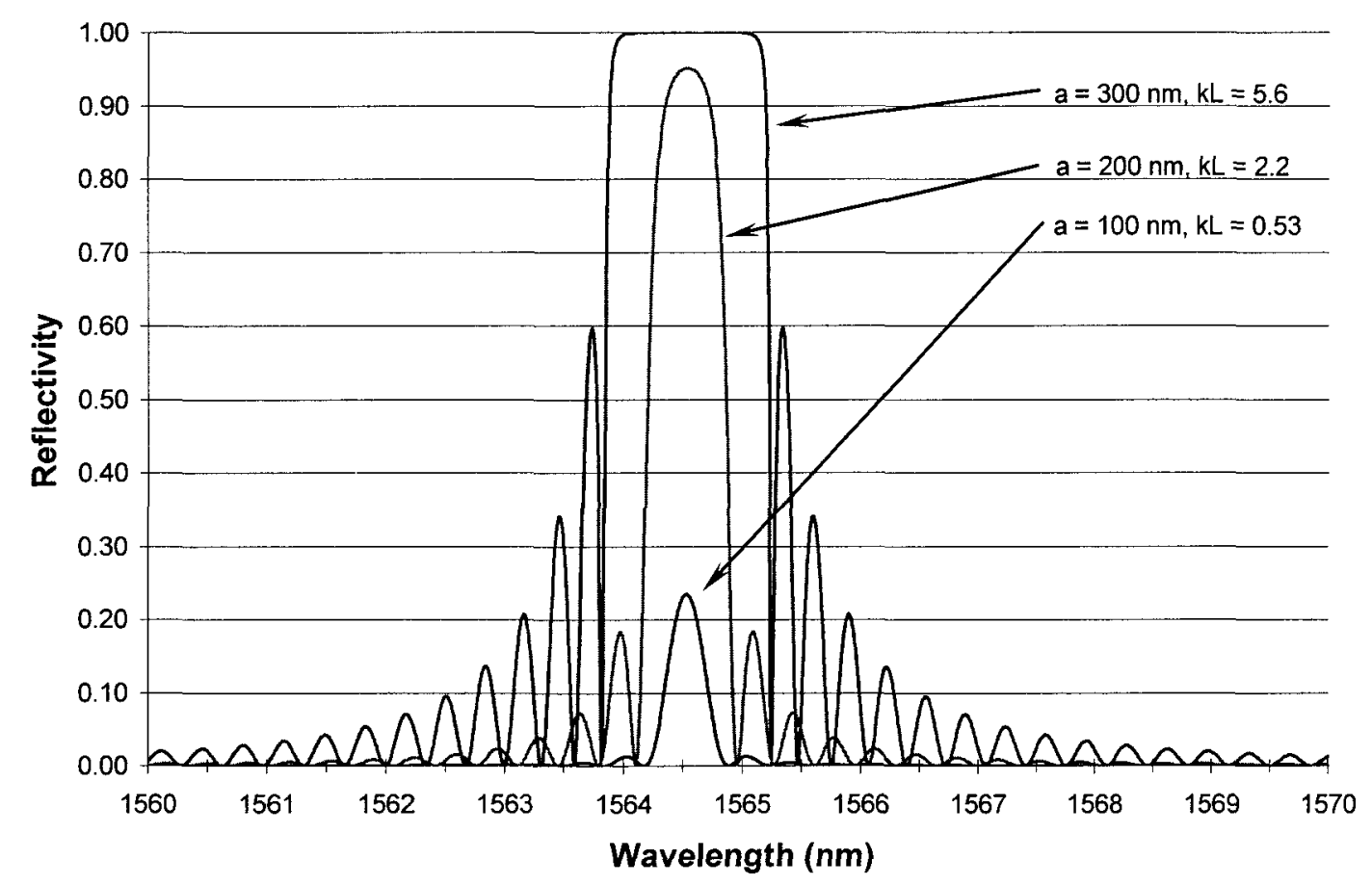

Figure 4.5. Reflectivity for different grating depths with a grating length $L_{B}=1000 \mu \mathrm{m}$

Analysis of grating design combinations provides a starting point for the other fabrication dimensions of the device. Gratings with a depth between 200 and $300 \mathrm{~nm}$ provide strong reflectance, but this must be balanced with the resulting increase in $\delta \lambda$. Should $\delta \lambda$ become too large, adjacent channel isolation becomes an issue. Increasing $L_{B}$ 
reduces $\delta \lambda$, which allows some compensation for increasing $a$, but $L_{B}$ is limited to half the overall length of the waveguide bridge. The length of the suspended waveguide bridge is limited by material and process factors that will be discussed later in the thesis.

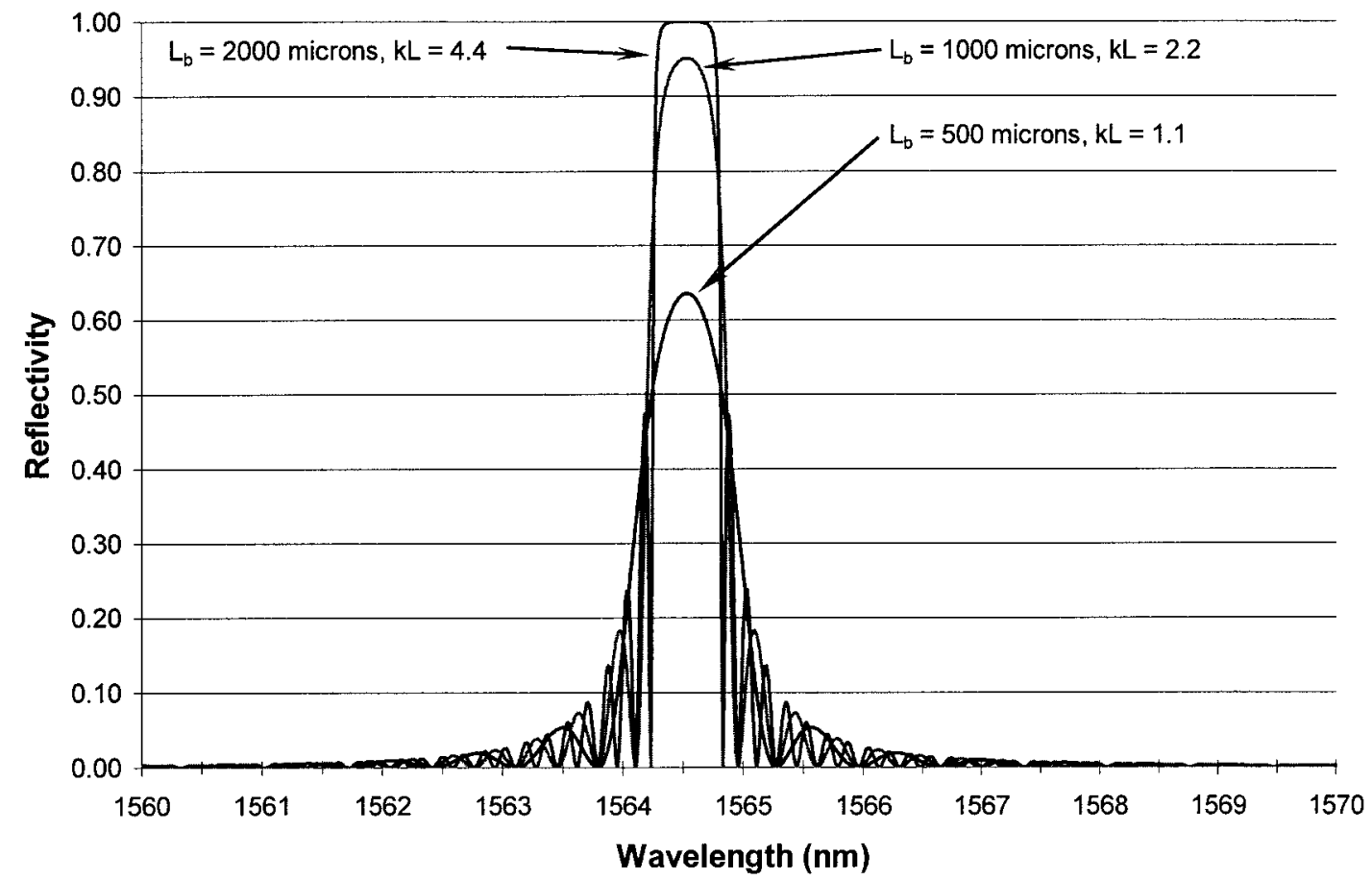

Figure 4.6. Reflectivity for different grating lengths with a grating depth $a=100 \mathrm{~nm}$.

\subsection{Heaters}

Thin film metallic resistors were chosen to develop the design and fabrication process for the integrated heaters of the device. Metallic heater strips, deposited on either side of the rib waveguide, were simpler to design, fabricate, and characterise than implanted and diffused semiconductor heaters.

An excellent material for use as the thin film heater is nichrome $(\mathrm{NiCr})$ as an $80 / 20$ percent alloy. This material has the benefit of a comparatively high resistivity for a metal, is a common material used in thin film deposition, and has high temperature and 
oxidisation resistance. As an example, given a heater element made of $\mathrm{NiCr}$ that is $2000 \mu \mathrm{m}$ long, $5 \mu \mathrm{m}$ wide and $200 \mathrm{~nm}$ thick; along with a resistivity of $110 \times 10^{-8} \Omega \mathrm{m}$ [90], equation 3.13 can be used to calculate a resistance value of $2200 \Omega$. This material also has good resistance to hydrofluoric acid (HF), which is used to etch the sacrificial BOX layer to release the device layer waveguide as a bridge.

Another material that is a good candidate for the heater design is gold. It is a common thin film deposition material and would not require a bond pad, leading to one less mask step in the fabrication process. It is also unaffected by HF. With a resistivity of $2.44 \times 10^{-8} \Omega \mathrm{m}$ [91] a heater with the same dimensions as the $\mathrm{NiCr}$ heater would have a resistance of $45 \Omega$.

A basic analytic comparison between the $\mathrm{NiCr}$ and gold heaters described above can be conducted in which they are required to produce $500 \mathrm{~mW}$ of total heating power. By fixing the power and resistance values, the $\mathrm{NiCr}$ heaters would require $15 \mathrm{~mA}$ at $33 \mathrm{~V}$, while gold heaters would require $106 \mathrm{~mA}$ at $4.7 \mathrm{~V}$. These current and voltage levels are reasonably attained in a lab setting and are not a factor in the experimental stage of development. In the interest of attempting a low current device, $\mathrm{NiCr}$ was chosen for the first design run. If other factors such as electronic integration were to be considered, low voltage would make the gold heaters attractive. The potential for gold contamination would require isolation of the heaters from the electronic circuitry and processing at the back-end.

It is useful at this point to provide an estimate of the power that would be required to bring a $1000 \mu \mathrm{m}$ long Bragg grating filter, integrated at the centre of a $2000 \mu \mathrm{m}$ long suspended rib waveguide that resides in a vacuum cavity, to a maximum operating 
temperature of $100{ }^{\circ} \mathrm{C}$. The estimate will be for a $1 \mathrm{D}$ problem with heat transport by conduction only.

The conduction rate equation along the length of the Bragg grating can be written as:

$$
Q=\kappa A \frac{d T}{d x}
$$

where $Q$ is the heat transfer in watts, $\kappa=148 \mathrm{~W} / \mathrm{m} \cdot \mathrm{K}$ is the thermal conductivity of silicon [66], $A$ is the cross-sectional area of the rib waveguide bridge, $d T$ is the temperature change, and $d x$ is the distance along the Bragg grating from the centre. If $\mathrm{T}_{\infty}=22^{\circ} \mathrm{C}$ then $d T=78^{\circ} \mathrm{C}$. The cross-section area of the rib waveguide is $128 \times 10^{-12}$ $\mathrm{m}^{2}$ and the Bragg grating filter that must be heated to $100{ }^{\circ} \mathrm{C}$ is $1 \times 10^{-3} \mathrm{~m}$ long. This results in a power requirement of $5.91 \mathrm{~mW}$. When other factors such as conduction to the air surrounding the bridge and to the bulk handle layer silicon below are taken into account, the value will increase. This approximation gives a basic estimate of the low power required for the device to function, particularly if highly efficient diffused resistive heaters and hermetic packaging are used.

\subsection{Thermally Tuning the Bragg Filter Wavelength}

To develop a thermomechano-optical model to describe the change in Bragg wavelength as the temperature changes, the thermal influence on the thermo-optic, stress-optic, and thermo-mechanic effects defined in chapter 3 need to be factored into equation 3.6 to produce equation 4.3 . 


$$
\Delta \lambda_{B}=\left(2 \frac{d n_{e f f}}{d T} \Lambda+2 n_{e f f} \frac{\Delta \Lambda}{d T}\right) \Delta T
$$

In the case of a thermally tuned FBG filter, in which the fibre is free to expand as it is heated, axial stress will not build up in the fibre and the device is sensitive to strain and temperature throughout its range of operation. In this example equation 3.6 can be expanded to [92]:

$$
\Delta \lambda_{B}=2\left(\Lambda \frac{\partial n_{e f f}}{\partial l}+n_{e f f} \frac{\partial \Lambda}{\partial l}\right) \Delta l+2\left(\Lambda \frac{\partial n_{e f f}}{\partial T}+n_{e f f} \frac{\partial \Lambda}{\partial T}\right) \Delta T
$$

where the first term of the equation represents the strain effect on the fibre and the second term represents the temperature effect on the fibre.

The device described in this thesis is a stiff suspended waveguide filter that is fixed or built-in at each supported end. Unlike the thermally tuned FBG, there will be three distinct regimes in which to calculate $\Delta \lambda_{B}$. In the pre-buckle regime, $n_{e f f}$ is sensitive to the temperature and stress within the device structure, and $\Lambda$ is not affected by strain. At the critical buckle temperature, when the waveguide bridge deflects to its buckling mode shape, $n_{\text {eff }}$ is sensitive to stress and $\Lambda$ is sensitive to strain. In the post-buckle regime, $n_{\text {eff }}$ is sensitive to temperature while $\Lambda$ is sensitive to strain. Instead of a unifying equation like 4.4 , it will be necessary to separately analyse the thermomechano-optical behaviour of the device in each regime of operation.

\subsubsection{Analysis of Bragg Wavelength Shift in the Pre-Buckle Regime}

During the pre-buckle regime of operation, as the device temperature builds towards the critical buckling temperature, the device is subject to thermal strain and an opposing axial compressive stress. Since the fixed-fixed suspended rib waveguide will 
not expand in the axial direction, the pitch of the Bragg grating filter will not change during this regime of operation. Therefore the shift in Bragg wavelength will occur only as a result of change in the effective index of the rib waveguide grating. This change will come as a result of the thermo-optic and photo-elastic effects.

The thermo-optic coefficient for the silicon of the rib waveguide, $d n_{\text {eff }} / d T$, is often set as a constant. In actuality the value changes as the temperature of the material changes. A second order polynomial interpolation for the TOC of silicon, valid from $300 \mathrm{~K}$ to $600 \mathrm{~K}\left(27^{\circ} \mathrm{C}\right.$ to $\left.327^{\circ} \mathrm{C}\right)$, was developed by F. Corte, et al. [64]:

$$
\frac{d n}{d T}=8.61 \times 10^{-5}+3.63 \times 10^{-7} T-2.07 \times 10^{-10} T^{2}
$$

For the purpose of analytic computation, this function for the TOC was used. The temperature dependence of the rib waveguide effective index was taken to be the same as that of bulk silicon; $d n_{e f f} / d T=d n / d T$.

The change in the effective index of the rib waveguide grating as a result of the photo-elastic effect was first described in equation 3.35:

$$
\Delta n_{\langle 110\rangle}=-\frac{1}{2}\left(n_{0}\right)^{3}\left[\sigma_{\langle 110\rangle}\left(\pi_{11}-\pi_{12}\right)\right]
$$

where the room temperature value for the effective index, $n_{e f f}$, (which is $n_{0}$ ) is set to 3.450 for a common starting point with the experimental measurements in chapter 6 . The value for the stress optic coefficient along the [110] direction of the waveguide bridge is $\left(\pi_{11}-\pi_{12}\right)=-14.4 \times 10^{-13} \mathrm{~Pa}^{-1}[73]$. The axial compressive stress along the waveguide bridge, $\sigma_{\langle 110\rangle}$, as a result of thermal expansion was first introduced in equation 3.39:

$$
\sigma_{\langle 110\rangle}=-\alpha \Delta T E_{\langle 110\rangle}
$$

The modulus of elasticity, E, of single crystal silicon in the $\langle 110\rangle$ direction was 
determined to be $168 \mathrm{GPa}$ using equation 3.21. As with the thermo-optic coefficient, the thermal expansion coefficient, $\alpha$, is temperature dependant. Equation 4.6 describes a curve fit of the linear thermal expansion coefficient for silicon from $300 \mathrm{~K}$ to $1500 \mathrm{~K}$ $\left(27^{\circ} \mathrm{C}\right.$ to $\left.1227^{\circ} \mathrm{C}\right)[65]$ :

$$
\alpha=3.725 \times 10^{-6}\left[1-\exp \left(-5.88 \times 10^{-3}(T-124)\right)\right]+5.548 \times 10^{-10} T
$$

Combining equations 3.35 and 3.39 yields a relationship for the photo-elastic change in effective index as a result of constrained thermal expansion in an unbuckled waveguide bridge:

$$
\frac{\Delta n_{e f f}}{\Delta T}=-\frac{1}{2} n_{0}^{3}\left[\alpha E_{\langle 110\rangle}\left(\pi_{11}-\pi_{12}\right)\right]
$$

It is now possible to calculate the pre-buckle thermal sensitivity of the Bragg wavelength from the thermo-optic and photo-elastic influence on the effective index of the rib waveguide grating, using equation 4.3. For this calculation, the room temperature $T_{0}=22^{\circ} \mathrm{C}$, and the change in temperature $\Delta T=1.0^{\circ} \mathrm{C}$. The pre-buckle Bragg pitch, $\Lambda=225 \mathrm{~nm}$ and $n_{e f f}=3.450$. Since the pitch does not change in the pre-buckle regime, $\Delta \Lambda=0$. Using equations 4.5 and 4.7 to calculate $\Delta n_{\text {eff }} / \Delta T$, and using this value with equation 4.3 , results in a thermal sensitivity of $\Delta \lambda_{B} / \Delta T=83 \mathrm{pm} /{ }^{\circ} \mathrm{C}$. This pre-buckle Bragg wavelength thermal sensitivity, as well as the buckle shift and the post-buckle sensitivity of the Bragg wavelength, are gathered into a single plot that is shown in figure 4.11 .

The shift due to the thermo-optic effect is dominant over the shift due to the photo-elastic effect. Omitting the photo-elastic effect results in a rate of $84 \mathrm{pm} /{ }^{\circ} \mathrm{C}$. While the thermo-optic effect accounts for over $99 \%$ of the influence on $n_{\text {eff }}$, the photo- 
elastic effect is significant because its influence builds cumulatively as the bridge is heated towards its critical buckle temperature. When the bridge buckles and the photoelastic effect due to that cumulative stress is suddenly released, its contribution to the Bragg filter wavelength shift will be much more influential at that temperature; the amount of which will be determined in the next section.

\subsubsection{Analysis of Bragg Wavelength Shift in the Buckle Regime}

Before analysis of the optical behaviour of the device in the buckle regime can begin, the critical buckle pressure, $P_{c r}$, generated by the critical buckle temperature, $T_{c r}$, must be determined. Given a fixed-fixed beam, $L_{0}=2000 \mu \mathrm{m}$, that is $30 \mu \mathrm{m}$ wide and $5 \mu \mathrm{m}$ thick; with an $890 \mathrm{~nm}$ deep, $5 \mu \mathrm{m}$ wide rib running along the centre; the moment of inertia, $I$, can be calculated using equation 3.51. This completes the geometric component of the analysis. The material property components, $\mathrm{E}$ and $\alpha$, were determined in the previous section.

Given geometries $I$ and $L_{0}$, and the material property E; $P_{c r}$ can be calculated using equation 3.47. By dividing $P_{c r}$ with the cross-sectional area of the rib waveguide bridge, the critical buckling stress, $\sigma_{c r}$ is calculated. Given material properties $\mathrm{E}$ and $\alpha$, and estimating a value for $\Delta T, \sigma_{c r}$ can also be approximated using equation 3.22. An iterative calculation of the difference between the two $\sigma_{c r}$ 's, that resolves the difference to zero, is used to determine the $\Delta T$ that will result in $\sigma_{c r}$. The fixed-fixed rib waveguide bridge described in this analysis will have a critical buckle temperature of $T_{c r}=69.0^{\circ} \mathrm{C}$. The critical buckle force and stress that exists in the suspended waveguide bridge at this thermal limit are $P_{c r}=3.33 \times 10^{-4} \mathrm{~N}$ and $\sigma_{c r}=2.61 \times 10^{6} \mathrm{~N} / \mathrm{m}^{2}$.

When the waveguide bridge buckles, the influence of the photo-elastic effect on 
the effective index of the Bragg grating in the rib waveguide is released. This was discussed briefly in section 3.7 and will be fully examined in the next section. In this transient buckle regime, not only is the cumulative influence of the photo-elastic effect on $n_{\text {eff }}$ released, but the grating pitch is expanded due to the buckling mode shape the waveguide bridge takes. Since the transient buckle deflection of the suspended waveguide bridge occurs at a specific critical buckle temperature, the shift in the Bragg filter wavelength during this regime is temperature independent.

To begin the analysis of $\Delta n_{\text {eff }}$ in the buckle regime, the cumulative thermal influence of the photo-elastic effect on $n_{e f f}$ at $T_{c r}=69^{\circ} \mathrm{C}$ and $\sigma_{c r}=2.61 \times 10^{6} \mathrm{~N} / \mathrm{m}^{2}$ is calculated to be $-7.7 \times 10^{-5}$. This is the theoretical value for $\Delta n_{\text {eff }}$ if the waveguide bridge buckles at an infinitesimally small temperature above $T_{c r}$. To base the analysis on the experimental conditions in chapter 6 , the resolution of the analytic model is set to $1.0^{\circ} \mathrm{C}$. The $1.0^{\circ} \mathrm{C}$ shift to $70{ }^{\circ} \mathrm{C}$ that is associated with the buckle transition from the pre- to post-buckle regime will shift $n_{\text {eff }}$ by $1.97 \times 10^{-4}$ as a result of the thermo-optic effect. By comparing this value to the cumulative $7.7 \times 10^{-5}$ photo-elastic change in $n_{\text {eff }}$ within the buckle regime, it is possible to see that the thermo-optic effect now only accounts for $72 \%$ of the influence on $\Delta n_{\text {eff. }}$ The total change in the effective index between the preand post-buckle regimes becomes $\Delta n_{\text {eff }}=7.7 \times 10^{-5}+1.97 \times 10^{-4}=2.74 \times 10^{-4}$. The prebuckle value of $n_{\text {eff }}$ at $T_{c r}$ has increased to 3.4587 from its room temperature value of 3.450 .

The analysis of the change in the Bragg grating pitch, $\Delta \Lambda$, in the buckle regime begins with equation 3.53 . With this equation the linear thermal expansion at the critical buckle temperature, $d x$, can be related to the waveguide bridge buckle height, $\delta$, at the 
centre of a beam of initial length, $L_{0}=2000 \mu \mathrm{m}$.

$$
\delta=\sqrt{\frac{d x 4 L_{0}}{\pi^{2}}}
$$

With $T_{c r}=69.0^{\circ} \mathrm{C}$, and $\alpha=2.881 \times 10^{-6}{ }^{\circ} \mathrm{C}^{-1}$ at $69.0^{\circ} \mathrm{C}$; the thermal strain, $\varepsilon_{T}$, is $1.355 \times 10^{-4}$. Since $\varepsilon_{T}=d x / L_{0}, d x=0.27 \mu \mathrm{m}$ and $\delta=14.8 \mu \mathrm{m}$.

With a physical value for $\delta$, the line integral described in equation 3.59

$$
L_{0}+\Delta L=\int_{0}^{L} \sqrt{1+\left[\delta \frac{\pi}{L_{0}} \sin \left(\frac{2 \pi x}{L_{0}}\right)\right]^{2}} d x
$$

can be solved for the buckling mode shape of the fixed-fixed beam, which in turn provides the expansion of the Bragg grating pitch. Numerical analysis is used to solve the integral and the change in the beam length is calculated to be $\Delta L=0.27 \mu \mathrm{m}$. This expansion due to the buckling mode shape line integral is the same value as the change in length due to thermal expansion. This conservation of length change over the enter beam is to be expected, but has now been verified using two independent means of analysis.

Next the analysis is completed for the centre portion of the beam where the Bragg grating is located. Therefore $L_{0}=1000 \mu \mathrm{m}$ and the thermal expansion is $d x=0.135 \mu \mathrm{m}$. This time equation 3.59 is solved between the limits of $500 \mu \mathrm{m}$ and $1500 \mu \mathrm{m}$ where the Bragg grating is located. The resulting $\Delta L=0.135 \mu \mathrm{m}$. This value is half the value when the full length of the beam was analysed and is again equal to the linear thermal expansion, $d x$.

Where the difference occurs is the expansion of the grating due to the buckling mode shape is not constant along the length of the bridge. The change in Bragg grating expansion along the buckling mode shape in this range is not constant as it is with linear 
thermal expansion. Depending on the location along the grating, the change in the Bragg grating pitch ranges from no expansion, to twice the change due to linear thermal expansion. By analysing the grating along the region of maximum buckle expansion, a grating with pitch, $\Lambda=225 \mathrm{~nm}$, spreads $\Delta \Lambda=0.061 \mathrm{~nm}$. The Bragg pitch at the end of the buckle regime, which is the beginning of the post-buckle regime, is $225.061 \mathrm{~nm}$.

The values for $\Delta \Lambda$ and $\Lambda$, when combined with $\Delta n_{\text {eff }}$ and $n_{\text {eff, }}$ are used in equation 4.3 to calculate the change in the Bragg filter wavelength between the pre- and postbuckle regimes, $\Delta \lambda_{B}=0.55 \mathrm{~nm}$. This shift is added to the plot in figure 4.11. The analytic result is based on the experimental conditions in chapter 6 , where the temperature was changed in approximately $1.0^{\circ} \mathrm{C}$ increments for the thermomechanooptical characterisation. If $\Delta T$ is infinitesimally small, $\Delta \lambda_{B}=0.46 \mathrm{~nm}$, and there is no thermo-optical influence on $\Delta n_{e f f}$.

\subsubsection{Analysis of Bragg Wavelength Shift in the Post-Buckle Regime}

After the transient shift in the Bragg wavelength at the critical buckle temperature, thermal tuning continues in the post-buckle regime. While the influence of the thermo-optic effect on the effective index of the Bragg filter will continue, the influence of stress on the effective index will no longer be present. To demonstrate this the various elements of stress in the bridge must be isolated and examined separately. These elements include residual, compressive, and bending stress; respectively: $\sigma_{r}, \sigma_{c}$, and $\sigma_{b}$. Residual stress is present at room temperature in the film that forms the microstructure, and becomes evident upon its release from the sacrificial layer. A released cantilever, for example, will curl up, down, or remain straight depending on the whether the residual thin film stress is tensile, compressive, or neutral. This is shown in 
figure $4.7[93]$.

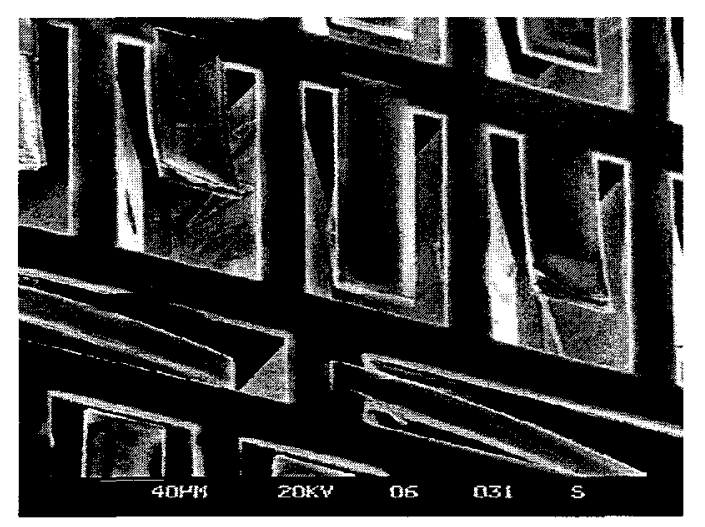

Figure 4.7. Examples of tensile, compressive and neutral stress on micromachined $\mathrm{SiO}_{2}$ cantilevers [93].

The suspended waveguide bridge structures proposed in this thesis are very long and slender. The high slenderness ratio results in a small $\sigma_{c r}$. Very little stress is required to deflect the waveguide bridge upon its release. SEM inspection at the centre of the bridge will indicate whether intrinsic stress is present in the bridge, once released.

Figure 4.8 depicts two $4000 \mu \mathrm{m}$ long, $5 \mu \mathrm{m}$ thick rib waveguide bridges, $\mathrm{A}$ and $\mathrm{B}$, after a successful sacrificial post-process release. Waveguide bridge A shows neutral intrinsic stress by remaining at the same distance above the handle layer of silicon once the sacrificial oxide has been removed. Waveguide bridge B demonstrates intrinsic compressive stress in the beam by deflecting slightly in-plane upon its release. Once this beam had been thermally actuated and forced to buckle out-of-plane, it remained in this second stable state, still showing deflection due to residual stress. This is an example of bi-stability. SEM metrology in the fabrication stage would ensure that only waveguide bridges with neutral residual stress would be thermomechano-optically characterised in the photonics lab. 


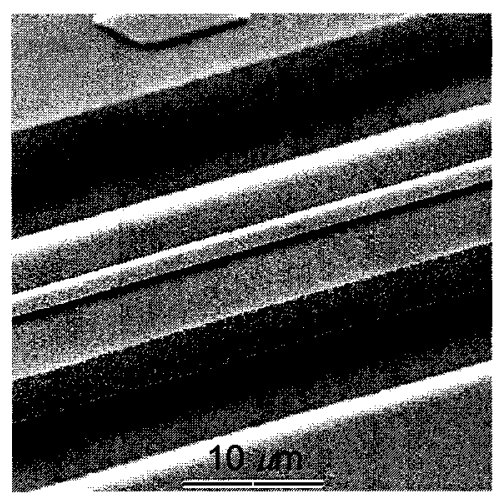

(A)

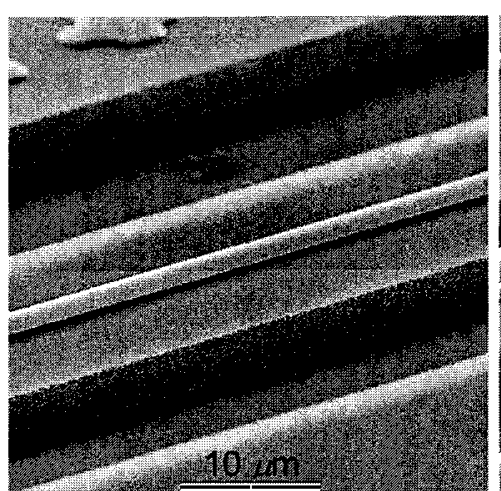

(B)

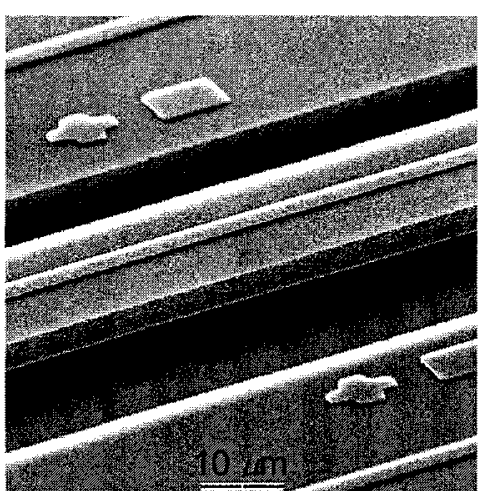

Figure 4.8. Two $4000 \mu \mathrm{m}$ long, $5 \mu \mathrm{m}$ thick waveguide bridges at room temperature.

Bridge A shows no residual stress. Bridge B shows bi-stable compressive residual stress.

In the pre-buckle range of operation, constrained thermal expansion of the fixedfixed suspended waveguide bridge causes axial compressive stress, $\sigma_{c}$, to steadily build in the structure until the critical buckling pressure, $P_{c r}$, is achieved. This stress is responsible for the photo-elastic influence on the effective index of the Bragg grating in the rib waveguide described in the previous section. When the waveguide bridge buckles, this compressive stress and its influence on the effective index is released.

According to stress theory for beams [94], once the waveguide bridge has buckled, bending stress develops. The stress will be zero along the centroid axis of the bridge. The location of this axis was defined with equation 3.50. Using the rib waveguide dimensions defined thus far for this design analysis, the centroid will occur close to the centre; at $2.86 \mu \mathrm{m}$ for a $5 \mu \mathrm{m}$ thick rib waveguide.

Bending stress during the post-buckle phase will be small due to the small deflection in relation to the beam length, and the magnitude will be approximately equal and opposite around the centroid. Therefore its post-buckle influence on the effective index of the Bragg grating is negligible. Figure 4.10 illustrates a beam in the pre and post-buckle positions with the associated stress distributions for $\sigma_{c}$ and $\sigma_{b}$. 


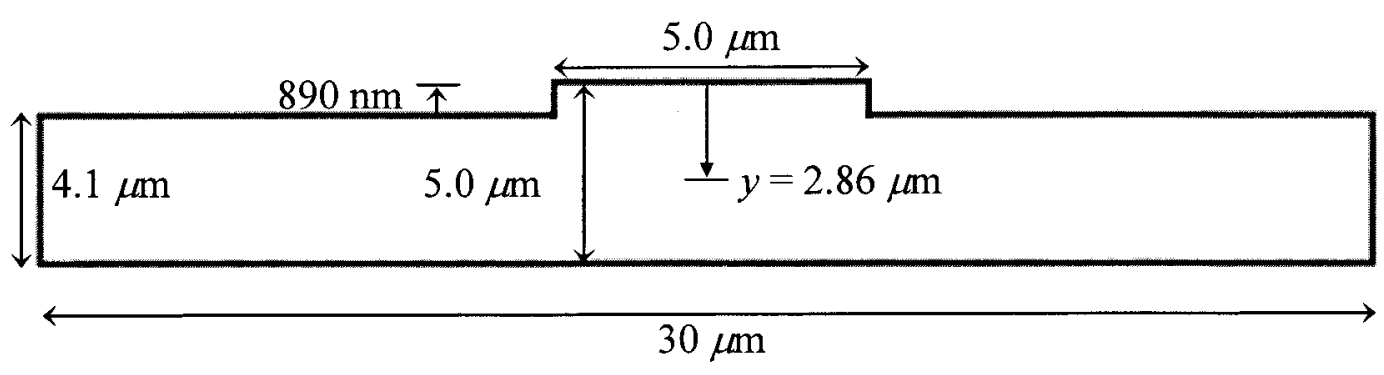

Figure 4.9. Location of centroid, $y$, in a rib waveguide bridge of specific dimensions.

With the waveguide bridge free to continue its out-of-plane deflection, the thermal strain will now have an effect on the grating pitch through the buckling mode shape. Although thermal expansion provides the force to compress the waveguide bridge into the shape of its new mode profile, it does not cause a broadening of the Bragg grating pitch. The change in grating pitch, $\Delta \Lambda$, is a function of the change in the bridge length, $\Delta L$, that the buckling mode shape causes. To calculate this effect equation 3.54 relates the thermal strain, $\varepsilon_{T}$, to $\delta$.

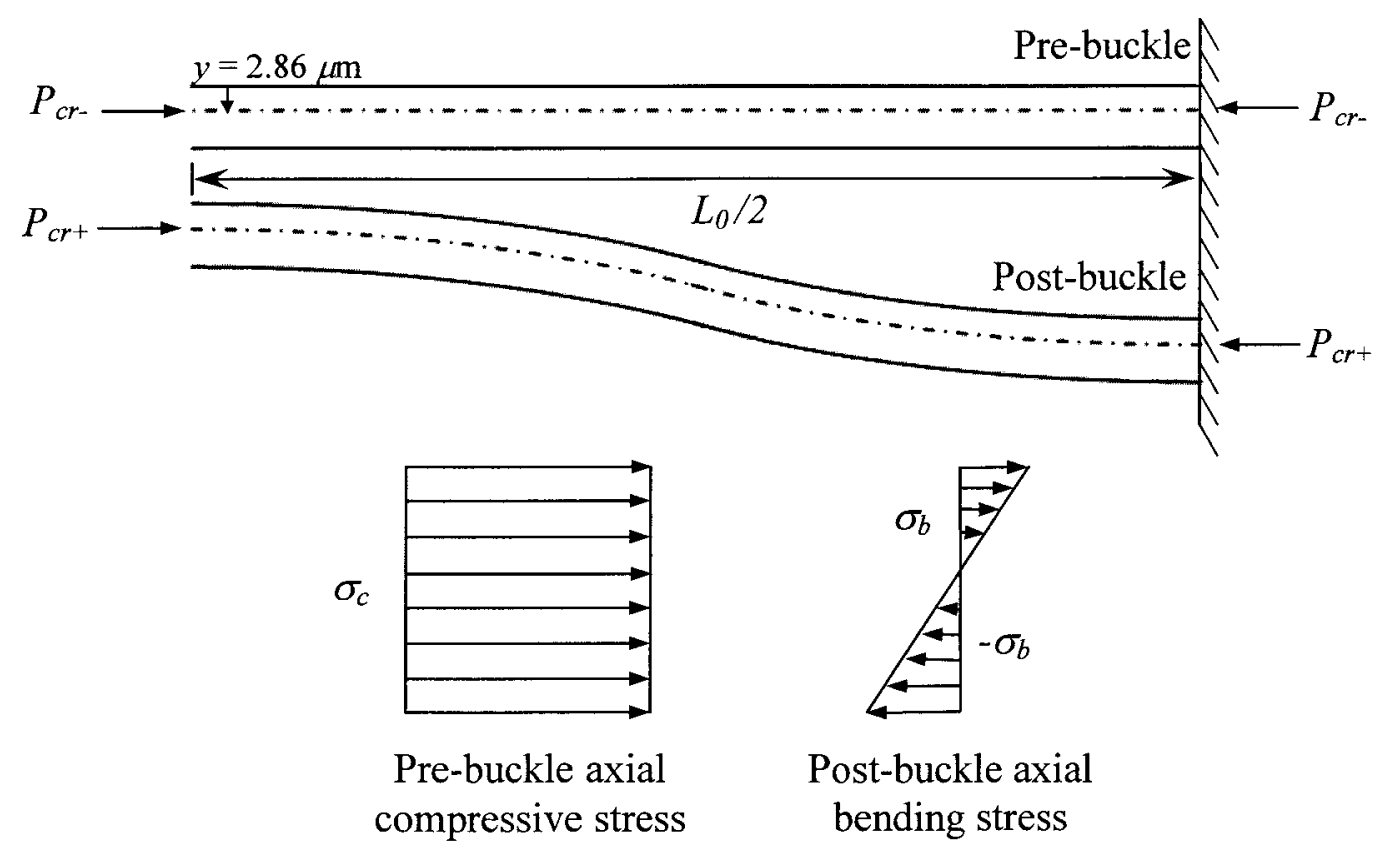

Figure 4.10. Lengthwise cross-section of bridge waveguide in pre- and post-buckle configurations with pre-buckle and post-buckle axial bending stress distributions. 


$$
\delta=\frac{2 L_{0}}{\pi} \sqrt{\varepsilon_{T}+\frac{\varepsilon_{T}^{2}}{2}}
$$

The thermal strain, $\varepsilon_{T}$, at the critical buckling temperature was calculated to be $1.355 \times 10^{-4}$ for a waveguide bridge of length $L_{0}=2000 \mu \mathrm{m}$ in the previous section. Using equation 3.54 results in $\delta=14.8 \times 10^{-6} \mathrm{~m}$, which is the same buckling mode deflection calculated separately with equation 3.53 . As the bridge continues to expand in the post-buckle regime, $\delta$ for a given temperature can be calculated, which in turn can be used to calculate the change in the grating pitch using equation 3.59 .

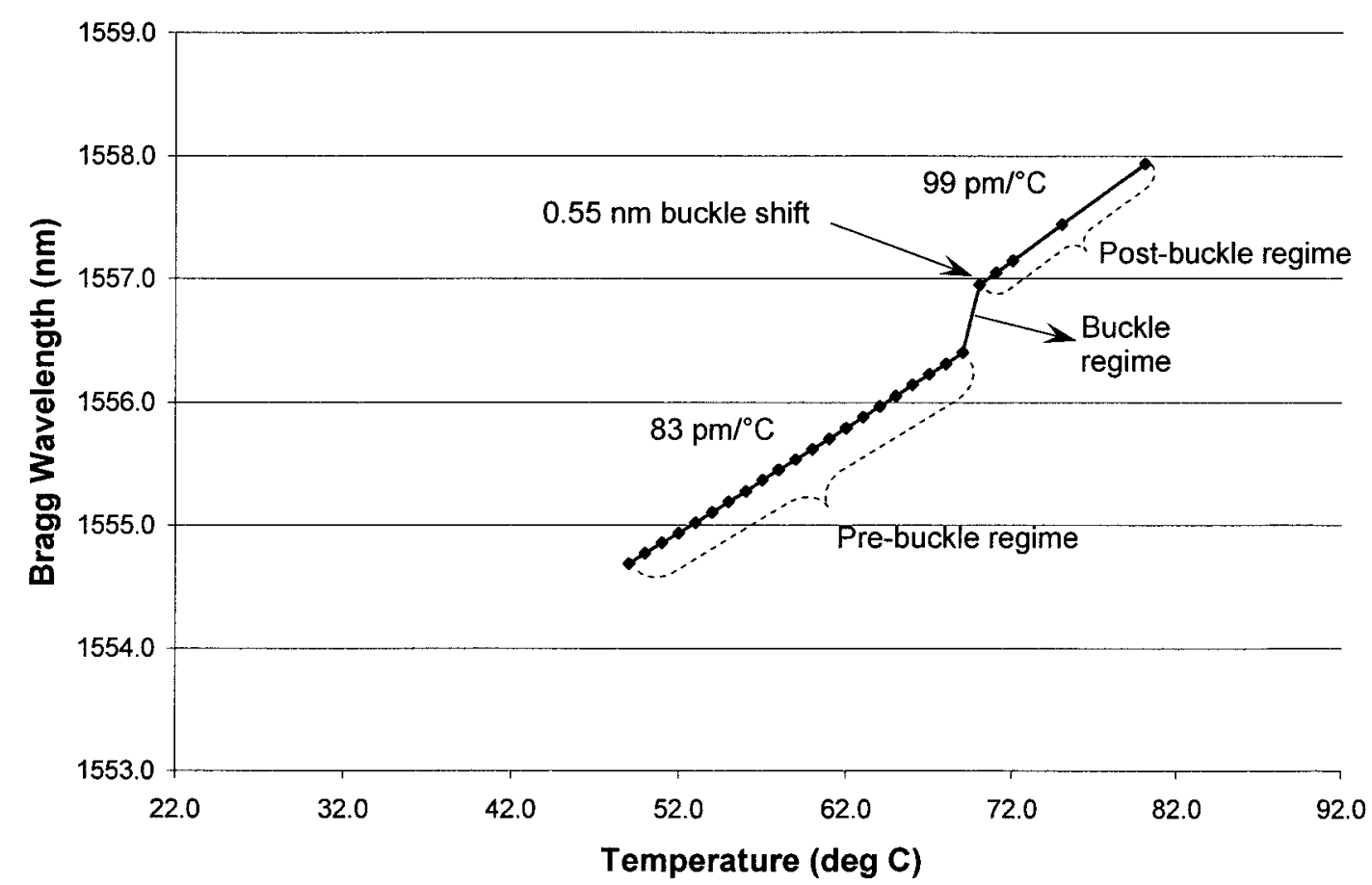

Figure 4.11. Theoretical plot of thermomechano-optical device behaviour as a result of analysis over the pre-buckle, buckle, and post-buckle regimes.

By combining $\Delta \Lambda$ with the thermo-optic influence on $\Delta n_{\text {eff }} \Delta \lambda_{B}$ can be calculated as the temperature changes in the post-buckle regime using equation 4.3 again. The resulting thermal sensitivity of the Bragg filter wavelength in the post-buckle regime is 
$\Delta \lambda_{B} / \Delta T=99 \mathrm{pm} /{ }^{\circ} \mathrm{C}$. This rate is added to the plot in figure 4.11 . This quantitative model can be compared to the qualitative model in figure 3.13 to give magnitude to both the buckle shift in the Bragg filter wavelength, and the thermal sensitivity in the pre- and post-buckle regimes.

\subsection{Mask design and layout}

Analysis of the optical, electrical, thermal, and mechanical elements of the proposed device provides a general understanding of the structural geometry required. With knowledge of the fabrication process capabilities available, masks can be designed to realize that geometry. The first design was called "proof of concept" or PC. This design used an existing Bragg grating and rib waveguide mask to establish the baseline process flow and optical characterisation techniques that would develop over the course of the research. The next design was called "Device 1" or D1. New masks were developed for an integrated heater and bridge that fit the geometry of the existing Bragg grating and rib waveguide mask.

The longest bridge length possible with the available mask making process was $4000 \mu \mathrm{m}$, corresponding to a $2000 \mu \mathrm{m}$ long grating. The shortest grating on the Bragg mask was $1000 \mu \mathrm{m}$, corresponding to a $2000 \mu \mathrm{m}$ bridge. In addition to these $4000 \mu \mathrm{m}$ and $2000 \mu \mathrm{m}$ bridge lengths, a variety of lengths from $100 \mu \mathrm{m}$ to $4000 \mu \mathrm{m}$, without a corresponding Bragg grating, were included in the bridge mask to help develop the bridge release post-process.

There were no pre-existing alignment marks on the PC Bragg grating mask to aid in centring the D1 heater and bridge masks along their length. Alignment windows were 
added to the D1 mask set to aid in backwards compatibility to the PC masks. Figure 4.12 depicts the heater and bridge masks, while figure 4.13 shows a SEM micrograph of the heater and bridge alignment windows in an actual device. A bond pad mask was also developed for this mask set.

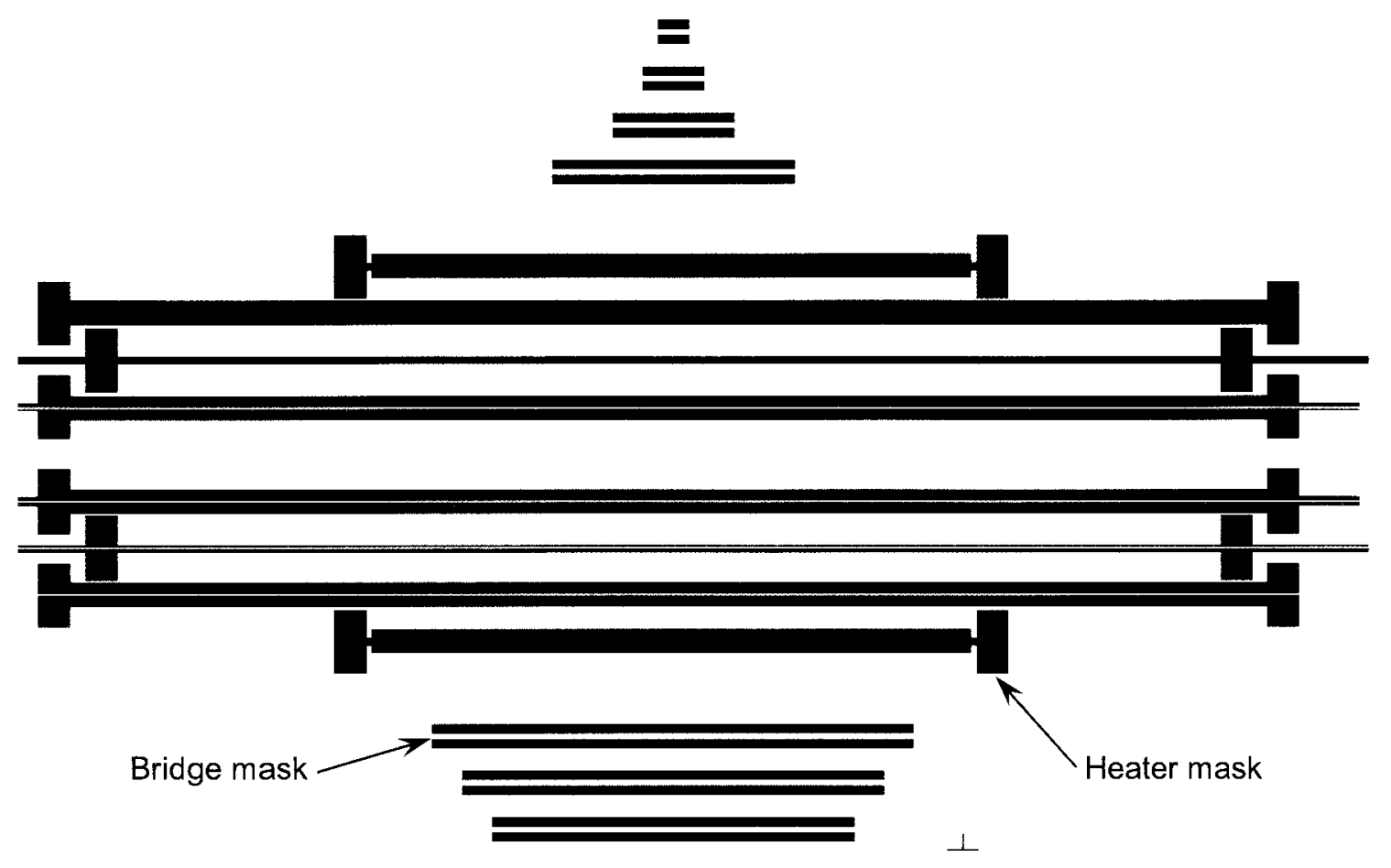

Figure 4.12. Partial D1 mask set including the heater mask and bridge mask.

The results from the fabrication and characterisation of the D1 device stream were used to iteratively advance the design and process development for the "Device 2" or D2 mask set, shown in figure 4.14. With a better understanding of what bridge lengths were suitable for the device, the selection was reduced to $500 \mu \mathrm{m}, 1000 \mu \mathrm{m}, 2000 \mu \mathrm{m}$ and $4000 \mu \mathrm{m}$. The resulting five mask set included a Bragg grating, rib waveguide, bridge, heater, and bond pad mask. Although not shown in figure 4.14 of the D2 mask set, the bond pad mask had long thin exposure windows to allow for better access to the lift-off resist during the lift-off process. 


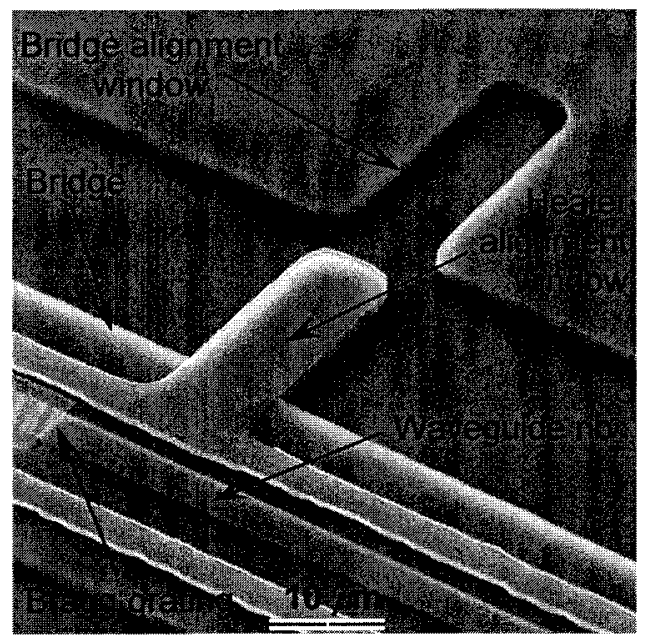

Figure 4.13. Alignment windows to assist in centring the $2000 \mu \mathrm{m}$ and $4000 \mu \mathrm{m}$ long bridge and heater masks along the PC grating mask in device D1.

The original waveguide mask was reused in the D2 development stream. Standard cross-in-box alignment marks were used to align the bridge, heater and bond pad masks to the new Bragg grating mask. The resulting suspended rib waveguide filters that would be used for thermo-mechanical and thermomechano-optical testing are labelled in figure 4.14. They include devices with or without integrated heaters, and with or without integrated Bragg gratings. If integrated heaters were used for the thermomechano-optical characterisation, two devices at each bridge length would be available on a die. If external heating was used there would be three devices at each bridge length available on a die.

Figure 4.15 shows a close-up of device variations for $500 \mu \mathrm{m}$ bridge lengths from the mask set in figure 4.14. The variations are intended to anticipate the range of operational parameters that might be of interest to isolate and characterise during the experimental analysis. These parameters are summarised in table 5. 


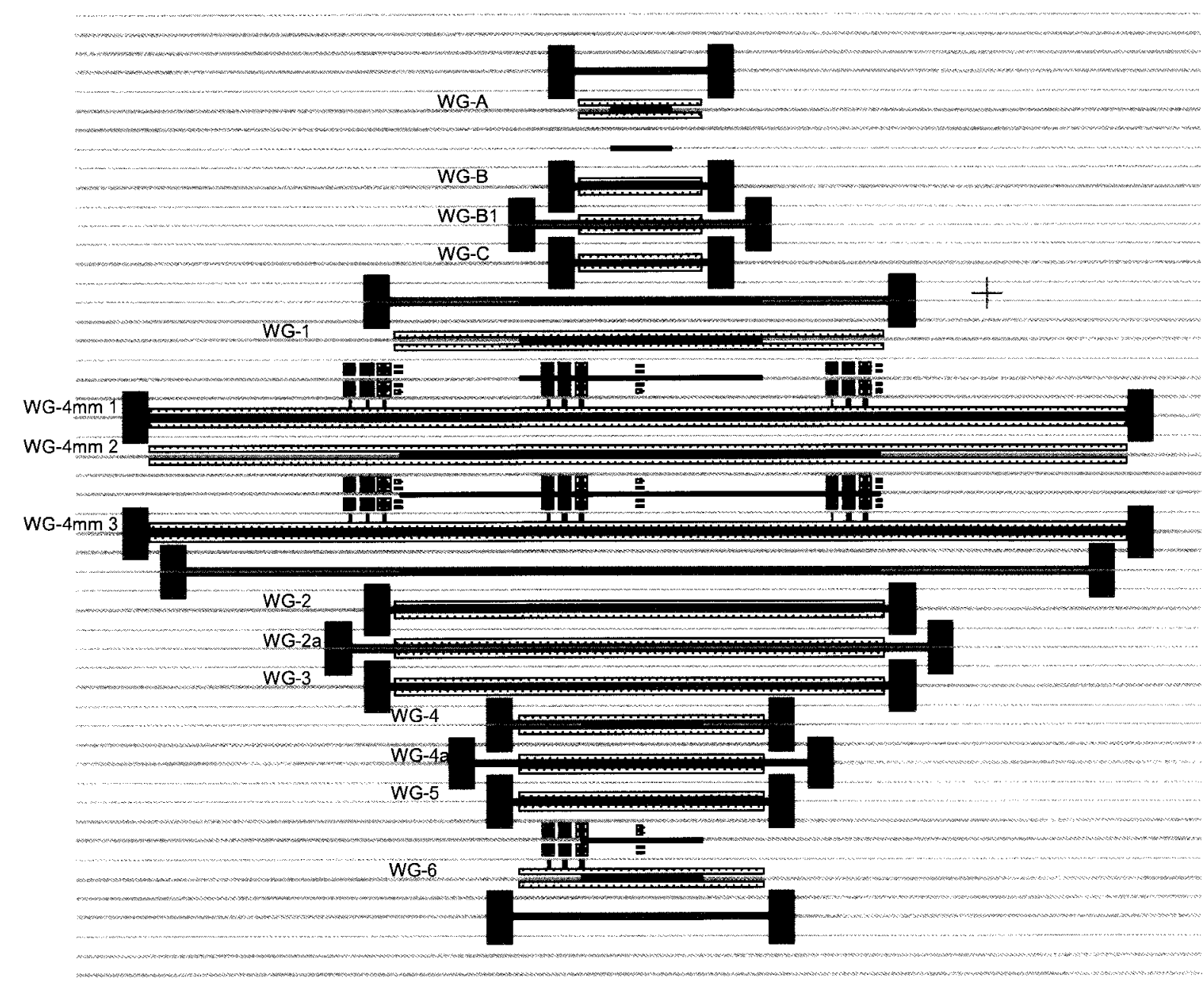

Figure 4.14. D2 mask set including the Bragg grating, rib waveguide, bridge, and heater masks for $500 \mu \mathrm{m}, 1000 \mu \mathrm{m}, 2000 \mu \mathrm{m}$, and $4000 \mu \mathrm{m}$ bridge lengths.

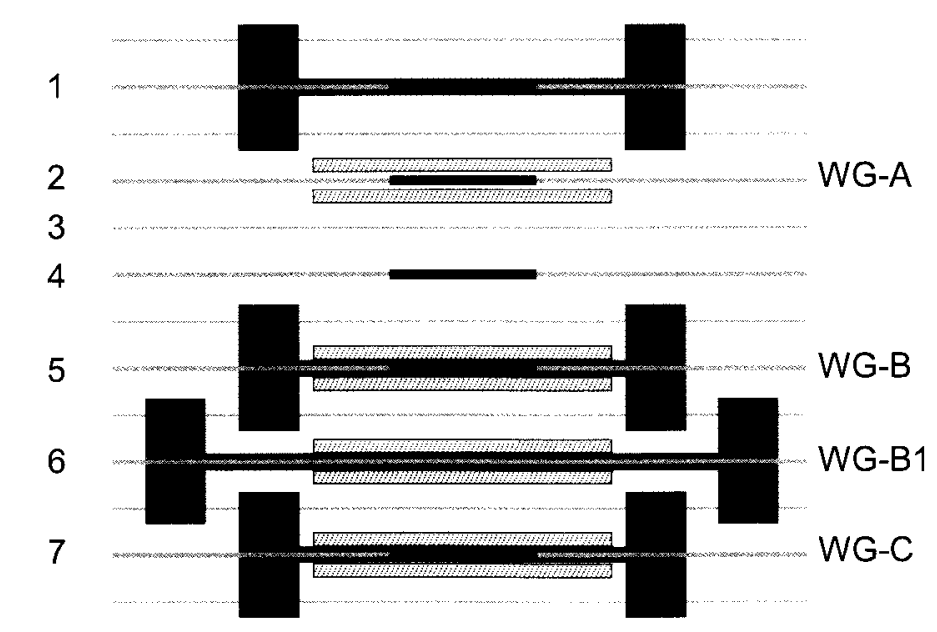

Figure 4.15. The $500 \mu \mathrm{m}$ bridge set showing device variations. 


\begin{tabular}{|c|l|}
\hline $\begin{array}{c}\text { Waveguide } \\
\text { Number }\end{array}$ & \multicolumn{1}{c|}{ Operational Parameter to Characterise } \\
\hline 1 & Thermo-optical measurement with integrated heater \\
\hline 2 & Thermomechano-optical measurement with external heater \\
\hline 3 & Baseline rib waveguide loss measurement \\
\hline 4 & Baseline Bragg filter measurement \\
\hline 5 & Thermomechano-optical measurement with integrated heater \\
\hline 6 & Thermo-mechanical bridge buckle measurement \\
\hline 7 & Thermomechano-optical measurement with integrated heater \\
\hline
\end{tabular}

Table 5. Summary of device variants for characterisation. 


\section{Fabrication}

We have the habit in writing articles published in scientific journals to make the work as finished as possible, to cover up all the tracks, to not worry about the blind alleys or describe how you had the wrong idea first, and so on. So there isn't any place to publish, in a dignified manner, what you actually did in order to get to do the work.

- Richard Feynman

Access to fabrication resources were available from three facilities. The first, and most important, was Carleton University's Microelectronics Fabrication Laboratory (MFL). The equipment in this fab was capable of accomplishing nearly all of the process steps described in this chapter. The exceptions were the Bragg grating mask lithography and initial portions of the waveguide bridge release post-process development. To achieve the submicron pitch required for the Bragg grating, direct-write electron-beam (e-beam) lithography was used. This process was performed in the Institute for Microstructural Sciences facilities at the National Research Council (IMS-NRC) in Ottawa, Canada. One of the techniques explored to release the waveguide bridges was critical point drying. These experiments took place at the McGill Microfab in Montreal, Canada.

Over the course of improving the process flow to fabricate the device described in this thesis, three devices were developed. The devices were called proof-of-concept (PC), device 1 (D1), and device 2 (D2) after the mask sets introduced in section 4.5. The PC run used an existing waveguide mask and SOI on which a Bragg grating mask had already been exposed and developed. The remaining process steps included a dry etch of the grating; exposing and developing the waveguide mask over the grating; etching the 
waveguide; and cleaving the waveguide facets. This process development was used to demonstrate an ability to fabricate and characterise a Bragg grating filter on SOI rib waveguides.

The next process run was developed to fabricate D1, a functioning thermomechano-optical device. By adding onto the PC process flow, the D1 process would integrate a heater for thermal tuning, and release the waveguide with integrated Bragg filter as a suspended bridge. The D1 process development made use of the remaining SOI wafer with the existing Bragg grating mask. New masks were designed for the heater and bridge elements that fit the geometry of the existing Bragg grating mask.

The results from fabrication and characterisation of the D1 device stream were used for design and process development of the D2 device. This stream used a new SOI wafer with thicker device and BOX layers, along with a new Bragg grating mask. New heater, bond pad, and bridge masks were also designed for this grating configuration.

The PC and D1 process development was completed on a single $100 \mathrm{~mm}$ SOI wafer. The D2 process development was completed on two $100 \mathrm{~mm}$ SOI wafers. The first wafer was used for device process development and the second wafer was used for bridge release post-process development. Due to the difficulty of processing the Bragg grating mask on an SOI wafer, and the cost of the wafers themselves, $7 \mathrm{~mm} \times 12 \mathrm{~mm}$ die were singulated after the Bragg grating mask was written. Process development was performed on these die. Process development is generally completed on no less than $50 \mathrm{~mm}$ wafers, therefore developing a process flow for up to five mask steps on these die was a challenge on its own; one that would require creative and meticulous handling 
techniques.

The following is a high level description of the process flow that was developed for the D2 stream. Figure 5.1 provides cross-sections of the main process gates. Subsequent sections in the chapter will provide a detailed description of the individual process steps.

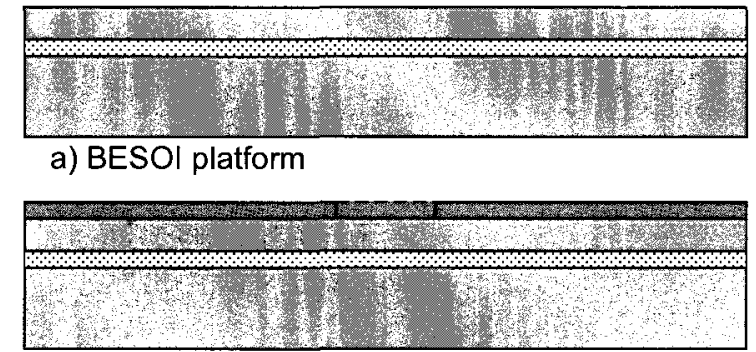

b) Bragg grating e-beam lithography
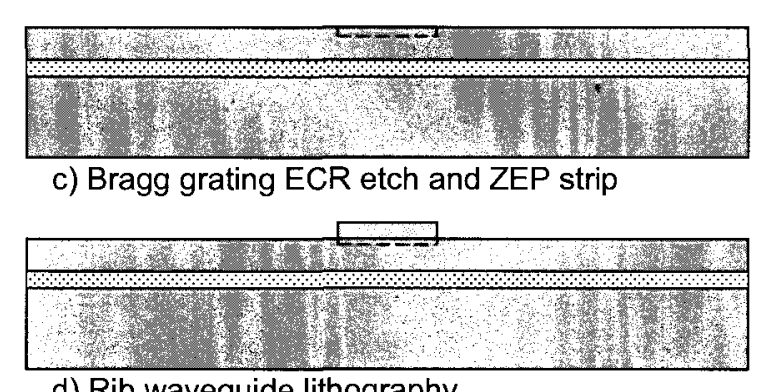

d) Rib waveguide lithography

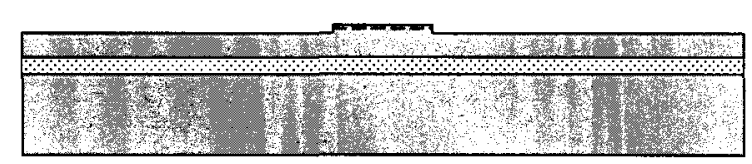

e) Rib waveguide ECR etch and PR strip

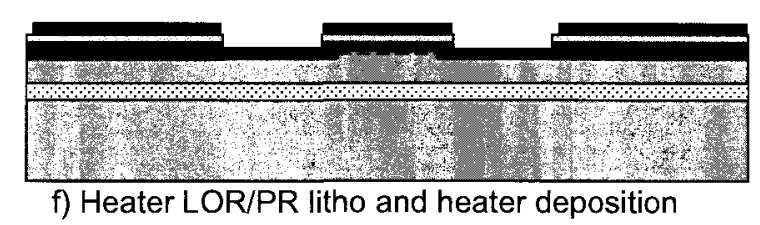

Silicon BOX

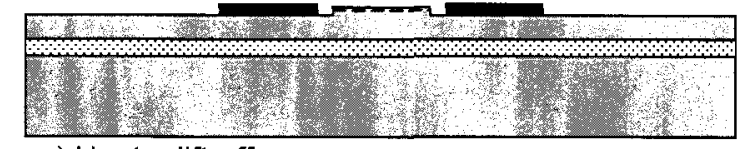

g) Heater lift-off

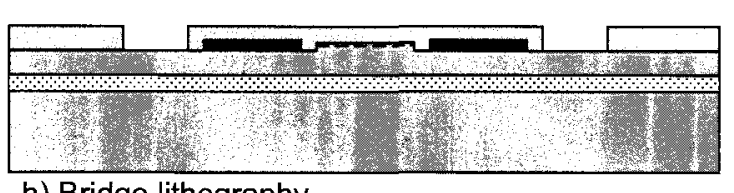

h) Bridge lithography

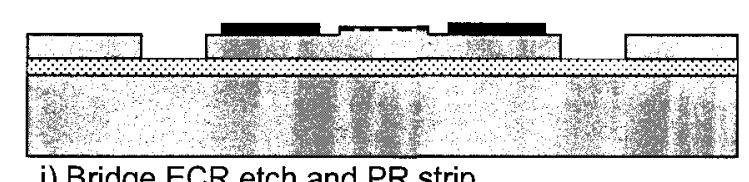

i) Bridge ECR etch and PR strip

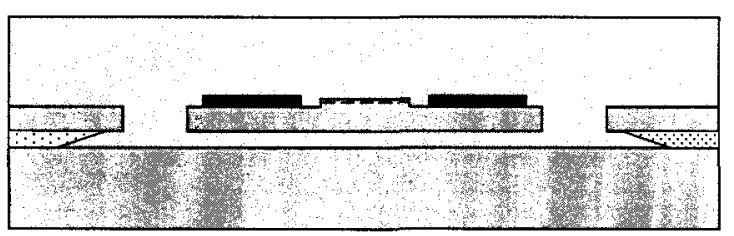

j) Bridge release wet etch w/ backfill PR support

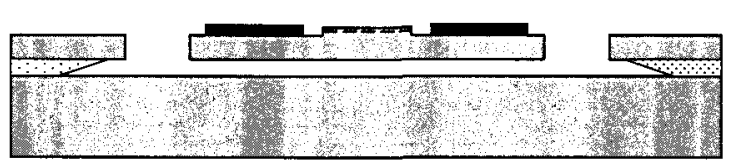

k) $\mathrm{O}_{2}$ plasma ash release of rib waveguide bridge

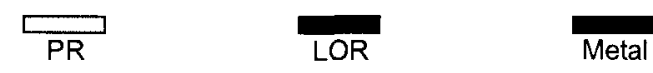

Figure 5.1. Process flow diagram for D2 fabrication stream.

The process begins with a BESOI (Bonded and Etched-back SOI) wafer from Ultrasil Corporation, featuring a $5.0 \mu \mathrm{m}$ device layer of single crystal silicon (SCS) and a $2.0 \mu \mathrm{m}$ buried oxide (BOX) layer of thermal oxide. E-beam lithography is used to pattern 
the Bragg grating mask in ZEP onto the device layer of the SOI, and an ECR RIE is used to etch the grating into the silicon. Next a rib waveguide mask is aligned along the Bragg grating and photolithographically defined in PR. The rib waveguide is etched with another ECR RIE step. LOR lithography is used to define the heater strips along either side of the rib waveguide and metal is deposited. After the LOR is removed, the bridge mask is photolithographically processed and etched to the BOX with a final ECR RIE step. Next the waveguide facets are cleaved. The final process step releases the bridge from the BOX using a sacrificial wet etch.

\subsection{Mask Generation and Lithography}

The bond pad, heater, and bridge masks that were designed and laid out for the D1 and D2 device streams were produced in the photomask generation facility at MFL. The Bragg grating mask was processed at IMS-NRC using e-beam lithography. The same rib waveguide mask was used for the $\mathrm{PC}, \mathrm{D} 1$, and $\mathrm{D} 2$ process development. This chrome mask was designed by Kevin Ramdas and Professor Garry Tarr at Carleton University for an electronically tunable Bragg grating filter in SOI [57].

\subsubsection{Mask Generation Process}

The mask generation process at Carleton University's MFL produces photo emulsion based masks. The mask patterns are exposed and developed on HTA Enterprises high contrast high resolution black and white photographic plates. These glass plates are either 2.5 inch or 4 inch square and are used with the mask aligner in the photolithographic process. 
The mask design process was outlined in section 4.5. The resulting native mask files are exported to Caltech Intermediate Form (CIF) files. The CIF file must then be transcoded to a proprietary command set for control and geometry that is used by the pattern generator (PG). This command set is called a mann file, named after David Mann, a designer of pattern generation equipment.

The first step to process a photomask is to load a photographic plate into the GCA Mann 1600A PG, shown in Figure 5.2. Motorized worm gears move the plate along the $\mathrm{x}$ - and $\mathrm{y}$-axis to zero the plate location. Next an arc lamp begins exposing the mask pattern in blocks along the $\mathrm{x}$-axis of the photographic plate. In this process the blocks had a minimum feature size of $50 \mu \mathrm{m}$. Once exposure along an axis is complete, the plate moves perpendicular, one exposure length along the $y$-axis, and the process is repeated along the next $\mathrm{x}$-axis. This rastering pattern was important to understand when defects began to show up in the exposure process.

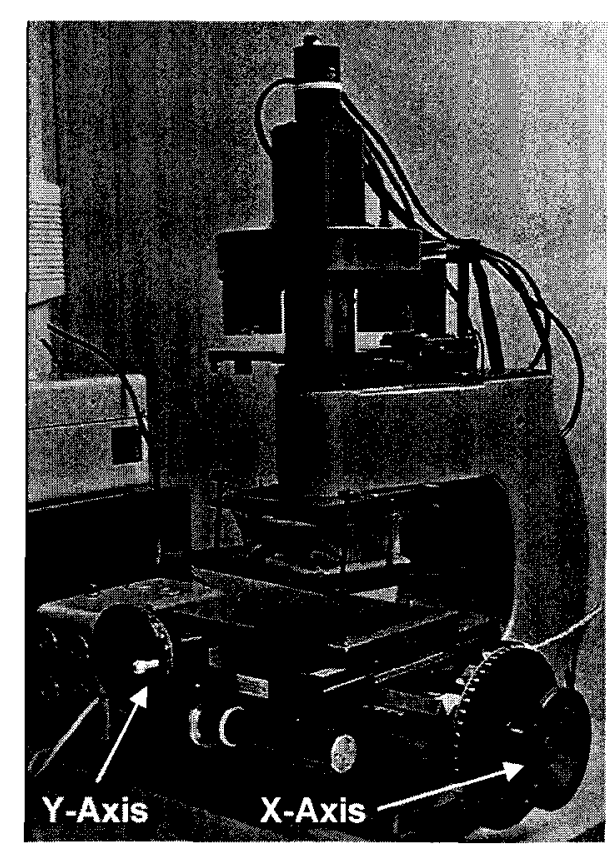

Figure 5.2. GCA Mann 1600A Pattern Generator. 
Once the PG exposure process is complete, the photographic plate is developed in a procedure similar to standard black and white photography. The run sheet for this procedure is found in appendix II. The result of the PG process is a clear field mask, a mask in which the emulsion film defines the pattern geometry and the majority of the surface is the clear glass of the photographic plate. The rib waveguide mask used throughout the process development was a clear field mask, with its chrome strips defining the masking area of the rib.

A dark field mask was used for the bond pad, heater, and bridge lithography. The absence of emulsion film on a dark field mask defines the pattern geometry to be processed photolithographically. To convert a clear field mask, generated by the PG, to a dark field mask requires further exposure steps. Figure 5.3 shows a clear field D2 heater mask from the PG compared to the final photoreduced dark field mask that is actually used in the photolithographic process.
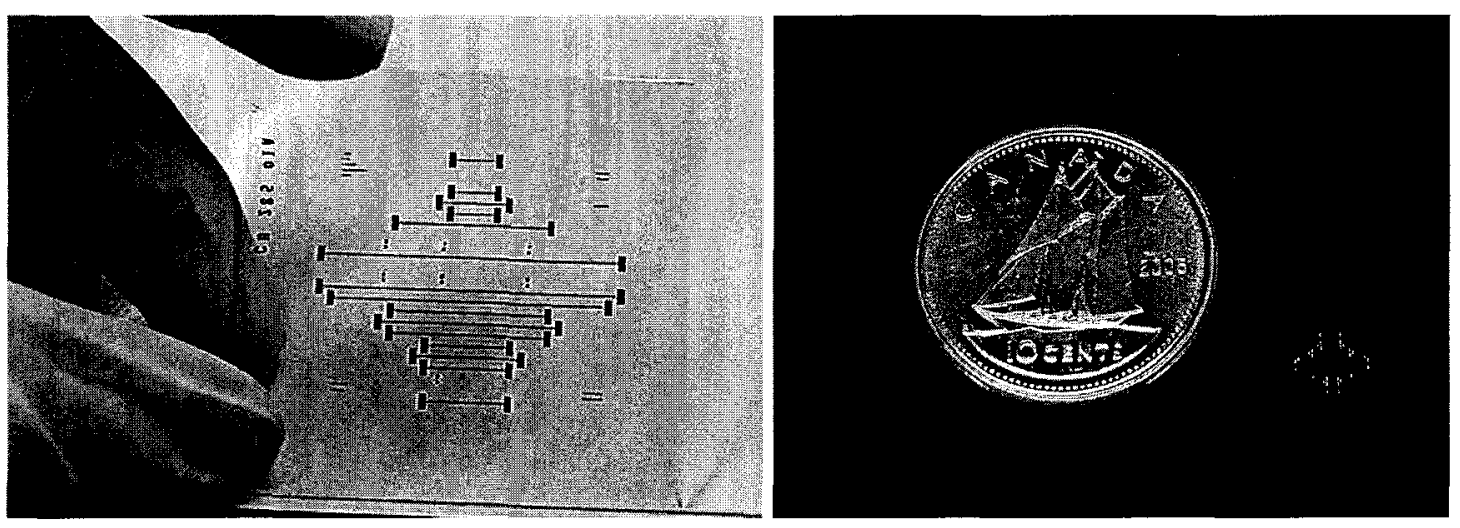

Figure 5.3. Clear field D2 heater mask from PG compared to final photoreduced dark field mask with a dime for scale.

Initial attempts to process the heater and bridge masks met with a misalignment of the exposure blocks that defined the $4000 \mu \mathrm{m}$ long emulsion strips, as shown in figure 5.4. Normally a misalignment of this sort is an indication that the optical registration grid 
used to sense the $x-y$ coordinates of the plate position is marked or smudged. This allows the position of the exposed blocks to become misaligned on the $x-y$ grid.

In this case, experimentation determined that the process of stepping the mask through the $x-y$ coordinates was such that only one block of each bridge or heater strip was exposed per $\mathrm{x}$-axis pass. This allowed systemic error to accumulate over the course of exposing only one block of the strips each x-axis pass. The error became visible for long thin exposure strips. By going back to the mask layout software and rotating the mask $90^{\circ}$ within the layout file, the $\mathrm{PG}$ would now expose the blocks defining an individual heater or bridge strip in one $\mathrm{x}$-axis pass, and the problem was resolved.

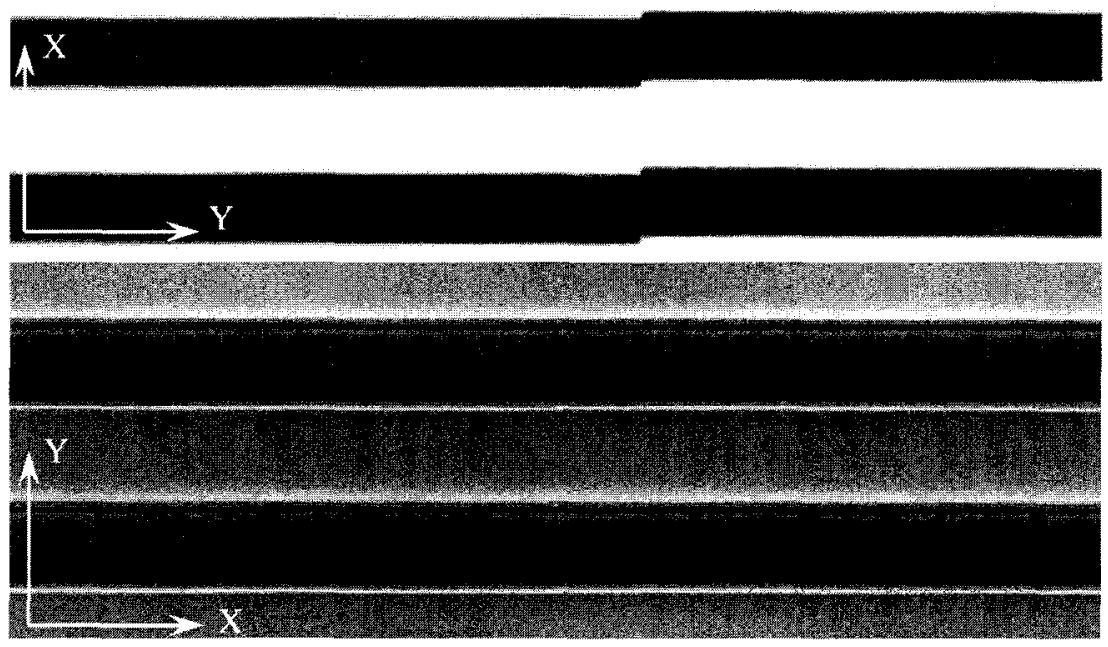

Figure 5.4. The exposure path is along the x-axis. Rotation of the heater mask within the mask file allowed a single strip to be exposed in a single pass along the $\mathrm{x}$-axis so that cumulative exposure alignment error would not occur.

Once the clear field mask master plate from the PG was completed, an Oriel 8410 photomask contact printer was used to expose a negative image of the mask. This contact printer, shown in figure 5.5, is used to make copies of masks as well as to convert a clear field to a dark field mask. The number of exposures required to make a dark field mask directly on the PG would be prohibitive compared to the single exposure required from 
the contact printer to reverse a clear field mask to a dark field mask. The resulting photographic plate was developed as before.

The next step in the mask generation process was to use the dark field mask in the Jade 4M-10AXYL step-and-repeat camera. This equipment, also shown in figure 5.5, creates a 10 times reduction of the mask it exposes. Therefore the $50 \mu \mathrm{m}$ minimum feature size of the master plate would become $5 \mu \mathrm{m}$. Once the $\mathrm{x}$-y controllers centred the plate under the camera, the master image was exposed and developed. The resulting plate would be a clear field mask again.
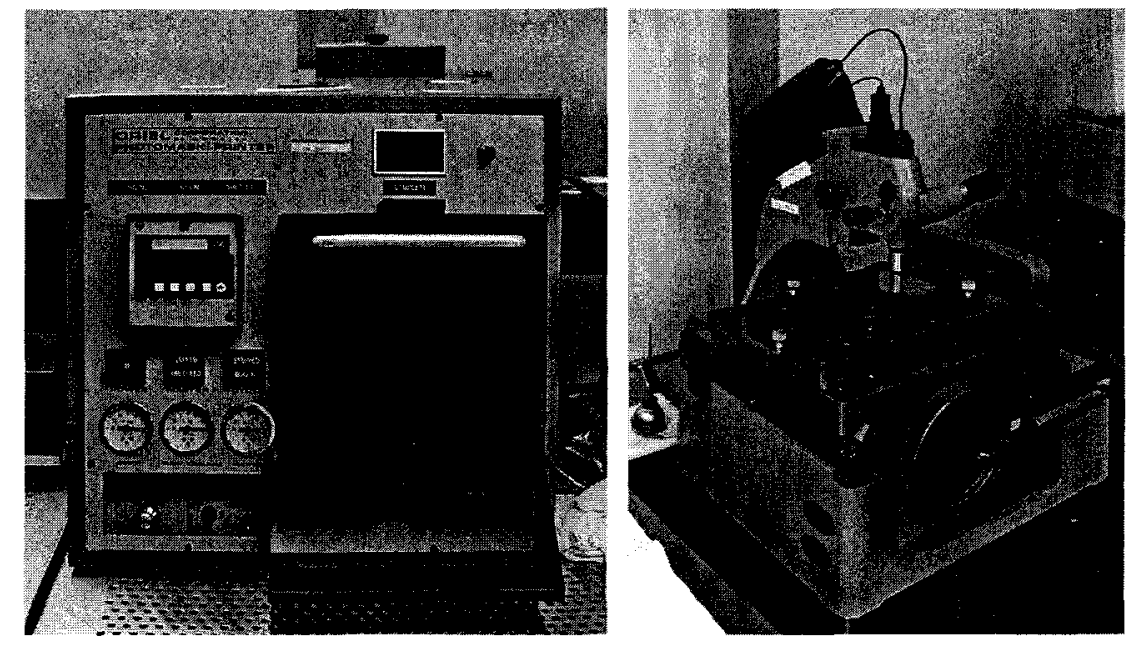

Figure 5.5. Oriel 8410 Photomask contact printer and Jade 4M- 10AXYL S\&R camera.

This plate was exposed in the contact printer to produce the final dark field mask. This production mask was inspected (as were all previous mask plates) to detect any imperfections or misalignments in the emulsion pattern. The final step in this process was to expose the pattern on a resist coated test wafer so it could be developed and inspected for the fidelity of the transferred pattern from the original mask layout. Figure 5.6 compares the mask layout file, resulting darkfield mask, and lift-off of the mask patterned in evaporated gold film. 


\begin{tabular}{|c|c|c|}
\hline ᄃ. АคUM & C. กคU & B. WOU \\
\hline H. TAIT & W. THI & NAI TI \\
\hline R. GAUTHIER & a 6aururs & 4. GAUTHIR \\
\hline CARLETDH UHIVERSITY & CAMLETOM ULYUES S & CARLETE UAYUEATYY \\
\hline J山и $/ 08$ & & มบแ \\
\hline
\end{tabular}

Figure 5.6. Comparison of the mask layout file, to dark field mask, to gold lift-off pattern with letter height of 25 microns.

\subsubsection{Electron Beam Lithography}

The $50 \mathrm{~nm}$ minimum feature size required to generate a $225 \mathrm{~nm}$ pitch Bragg grating pattern was too small for conventional optical lithography to resolve. E-beam lithography (EBL) was a comparatively inexpensive solution to pattern the Bragg grating mask. An e-beam direct write process had been developed and established at the MFL through previous research $[57,95]$. Without a precision stage for sample re-positioning, the maximum grating length that could be produced was $200 \mu \mathrm{m}$. The maximum grating length for the Bragg grating design in this research was $2000 \mu \mathrm{m}$.

The NRC-IMS facility operates a JEOL JBX-6000 direct write e-beam system with a precision stage that allows patterns to be written with a $25 \mathrm{~nm}$ resolution and a $60 \mathrm{~nm}$ overlay and stitching accuracy, using a $5 \mathrm{~nm}$ beam. This performance was within the limits required to write the $225 \mathrm{~nm}$ pitch Bragg grating over millimetre lengths, as described by the design. Once the design for the Bragg grating had been finalized and exported to a Graphics Data System (GDS) file, it was sent to Dr. Jean Lapointe, the scientist responsible for the EBL system at IMS.

IMS completed a standard RCA clean on the bare SOI wafer. This was followed 
by a dehydration bake, and an HMDS and ZEP520A e-beam resist spin coat. A preexposure bake was performed for 5 minutes at $180^{\circ} \mathrm{C}$. After the Bragg grating mask file was written, the pattern was developed in o-Xylene. The final film thickness was $3500 \AA$ with an interference colour of green.

\subsubsection{Photolithography}

Photolithography was used to transfer the waveguide, heater, bond pad, and bridge mask patterns onto a substrate coated by photoresist (PR). The Carleton Fabrication Lab possesses standard equipment for a photolithographic process flow. The equipment will be described in the order it was typically used. Detailed run sheets for each process are in appendix II.

Photolithographic processing takes place in a yellow room. Light of green wavelengths and shorter are filtered so the PR does not become exposed during handling. Of the two ventilated wet benches shown in figure 5.7, one accommodates alkaline based processes, such as developing; while the other houses acid based processes, such as liftoff and PR strip.

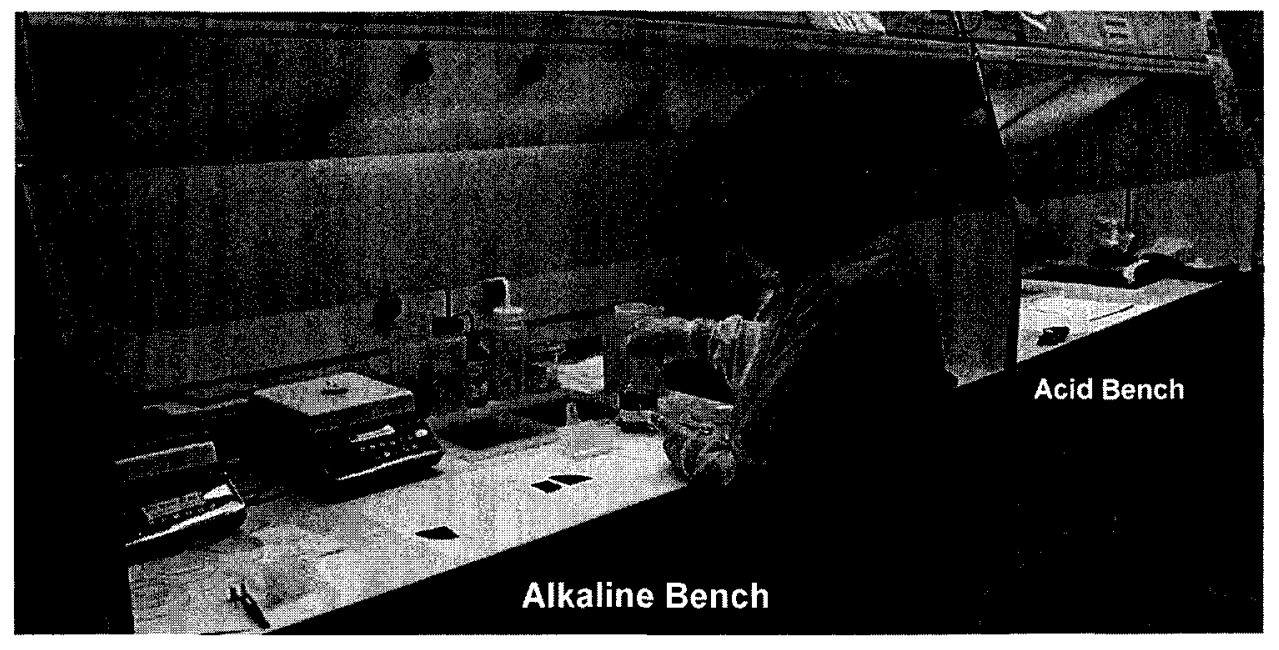

Figure 5.7. Alkaline and acidic wet benches in photolithography (yellow room). 
Temperature and humidity were recorded at the beginning of a process run. The yellow room in the Carleton fab was generally kept at $20^{\circ} \mathrm{C}$ and $40 \%$ humidity. When the air handling system allowed the humidity to rise above $55 \%$, PR adhesion would deteriorate enough to make the processing impractical.

A wafer or die would start the lithographic process with a dehydration bake in a Blue $\mathrm{M}$ oven at $200^{\circ} \mathrm{C}$ under a nitrogen flow. This would remove any moisture from the surface and promote adhesion of PR. Another method of surface preparation was the Plasma Preen plasma cleaning/etching system. It would perform a simultaneous dehydration and organics clean through the use of an oxygen plasma at temperatures in excess of $200^{\circ} \mathrm{C}$. Die would be isolated from the water-cooled base with ceramic standoffs. This system will be formally introduced in section 5.2 on dry etch processing.

Once the dehydration bake was complete, the next step was to spin hexamethyldisilazane (HMDS) onto the die and bake it at $105^{\circ} \mathrm{C}$ for one minute. HMDS combines with dangling $\mathrm{OH}$ bonds that dehydration leaves behind. Once the hydroxides have been neutralised on the oxide surface, the PR has an optimal surface to adhere to. The Solitec spin coater and dual hotplates are shown in figure 5.8 under a third fume hood. An HMDS primer oven was also available to maximize PR adhesion. This oven runs under a vacuum, and completes a series of binary cycles under vacuum and an HMDS vapour. 


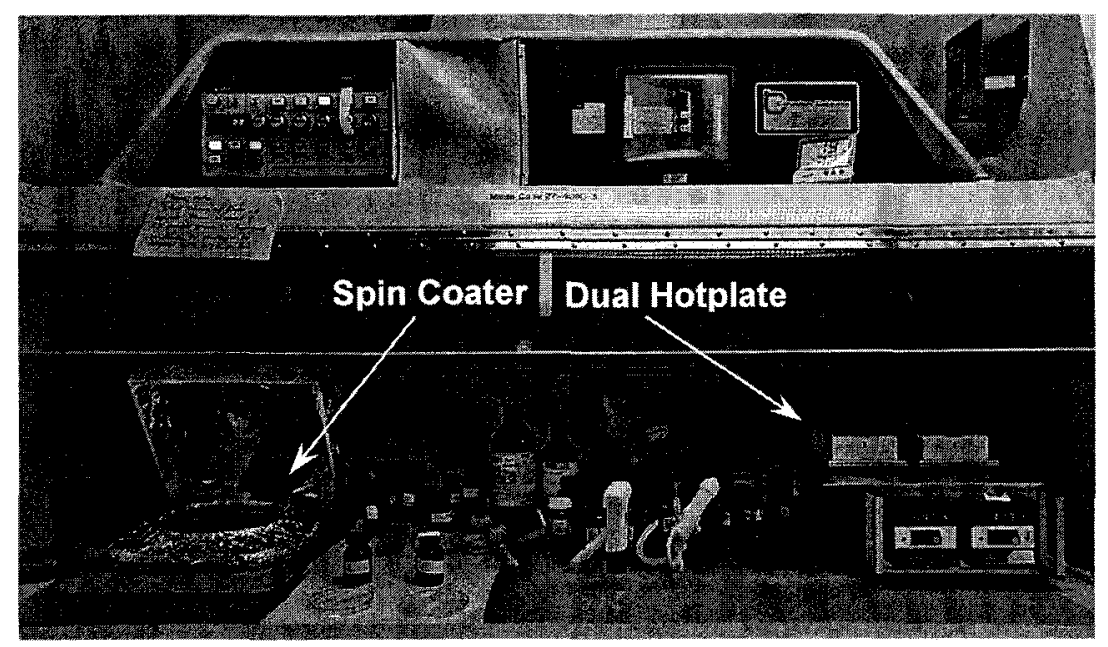

Figure 5.8. Spin coat bench with hotplates.

Given the small size of the process die, the standard spinner chuck with grooves for vacuum distribution could not be used. A special chuck with a single vacuum hole, shown in figure 5.9 , was used instead. This allowed the photolithographic process to be reliably performed on die as small as $6 \mathrm{~mm}$ square.

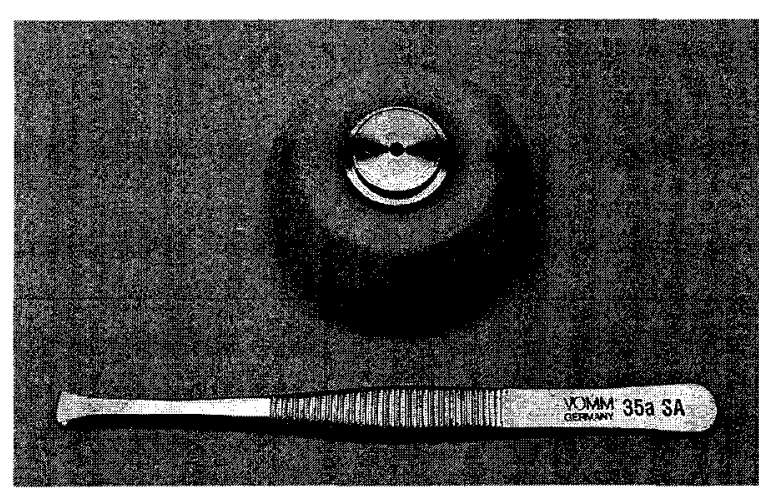

Figure 5.9. Single hole vacuum chuck for die as small as $6 \mathrm{~mm}$ square.

After the die surface had been properly prepared, PR was spun on. For most photolithographic work the Microposit S1800 series of positive photoresists were used; including S1805, S1811, and S1818. The higher the PR number, the thicker the resist would spin on at a given RPM. Different processes would demand different thicknesses. In the case where edge sharpness was important, a thinner resist was desired. In the case 
where resist was used in place of an oxide or metallic hard mask, or as a passivation layer, a thicker resist was desired. S1811 resist was a good compromise for all but the most extreme process conditions. After the spin coat was complete, the solvent that maintains the PR in liquid form was flash evaporated in a one minute soft bake at $105^{\circ} \mathrm{C}$.

Spinning the PR on the die uniformly distributes the film, but it also results in an accumulation of resist on the edge of the die called an edge bead. Depending on the size of the die, the edge bead that results from the spinning process might be large enough to keep the mask from close contact to the PR. This was particularly important for the waveguide lithography, where the mask needed to be in hard contact with the PR in order to ensure a sharply exposed edge. In order to manually remove the edge bead from a coated die, a stainless steel foil was placed next to the bead and a scalpel was used to scrape the bead off the die. The foil prevented the PR chips that flew off from resettling on the die and interfering with the lithographic process.

A mask pattern was exposed on PR using MFL's Karl-Suss MA6 contact aligner, operating at a $365 \mathrm{~nm}$ UV exposure wavelength. This machine is shown in figure 5.10 . To begin the process a mask is loaded on the vacuum maskholder and the PR coated die is placed on the vacuum chuck. The two are then brought into close proximity so that alignment between the mask and any existing patterns on the die can be made. Once the mask alignment is complete a high precision (HP) vacuum contact or standard program (ST) soft contact exposure is made. 


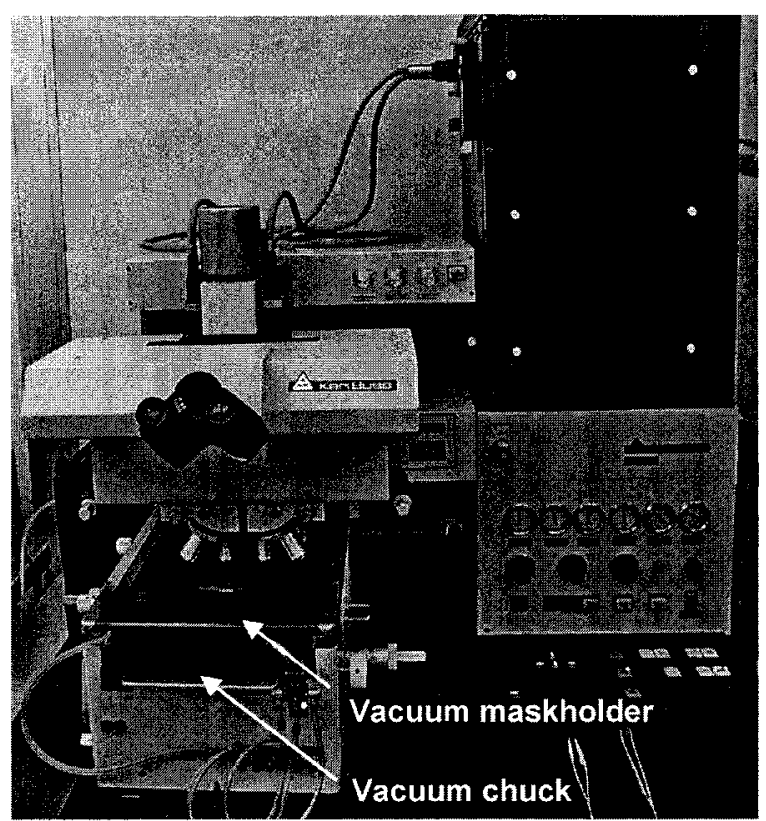

Figure 5.10. Karl-Suss MA6 contact aligner.

The HP mode turns off the chuck vacuum after the die and mask have been brought into contact. A second vacuum evacuates the space between the mask and wafer to draw them into hard contact. This setting is useful when a sharp exposure edge is desired. The ST setting maintains the chuck vacuum and an air gap remains between the die and mask. This setting is useful for a thicker resist coating or a lift-off resist process. Without this gap the die could stick to the mask surface, preventing fine alignment.

Once exposed, the die was developed in Shipley Microposit MF-321 developer. The duration was typically one minute, followed by a 45 second rinse in deionised water (DI). In the case where delicate structures were developed, such as the 3 to $5 \mu \mathrm{m}$ wide strips of the waveguide mask, care had to be taken when agitating the die in the developer as the structures could be toppled or segmented.

After the PR pattern had been developed, a final hard bake was necessary to drive off any remaining solvents and harden the PR mask. For S1811, 3 minutes at $125^{\circ} \mathrm{C}$ was 
typically used, but durations of up to 5 minutes and temperatures of up to $135^{\circ} \mathrm{C}$ were experimented with when PR was attempted for use as metal passivation with the HF bridge release process development. For a typical lithographic flow described up to this point, the PR thickness was nominally $1.4 \mu \mathrm{m}$. Measuring the thickness of the PR at this stage of the process flow was important when silicon to PR etch selectivity ratios were being determined.

If patterned die were to be left over night before the subsequent etch step, an additional 2 minute hard bake at $125^{\circ} \mathrm{C}$ was performed to drive off any accumulated moisture. In addition to its stated purpose, the hard bake step also causes PR to flow. This could lead to distortion of corners, diminishing edge sharpness, or reduction of coverage for step height geometries. Balancing these effects against bake time and temperature had to be weighed depending on the selectivity and duration of the etch step that would follow.

\subsubsection{Lift-off Resist}

A separate class of photoresists are lift-off resists (LOR). These resists were used as part of the metal deposition process. The thickness of the metal deposition would dictate what type of LOR was used; in this case, Microchem LOR 3A and 10B. Generally the target LOR thickness is $50 \%$ greater than the thickness of the film to be deposited. For films with a thickness less than $500 \mathrm{~nm}$, LOR $3 \mathrm{~A}$ was used. For thicker films in the one micron range, LOR 10B was used. When the mask is exposed on this bilayer LOR/PR film and developed, the pattern is transferred to both the LOR and PR. The LOR is more reactive to the developer, leaving the PR as an overhanging lip. For this process flow development, LOR was spun-on, soft baked for 5 minutes at $185^{\circ} \mathrm{C}$, 
then coated with S1811 PR, which was also soft baked.

The next step is the metal film deposition, which coats both the photoresist and the mask patterned substrate as shown in figure 5.11. The film deposition process will be formally introduced in section 5.3. After submersion in an organic solvent, the lift-off resist acts as a sacrificial layer, allowing the photoresist and the metal on it to be carried away. The metal pattern is left behind on the substrate in a somewhat "stencil-like" process.

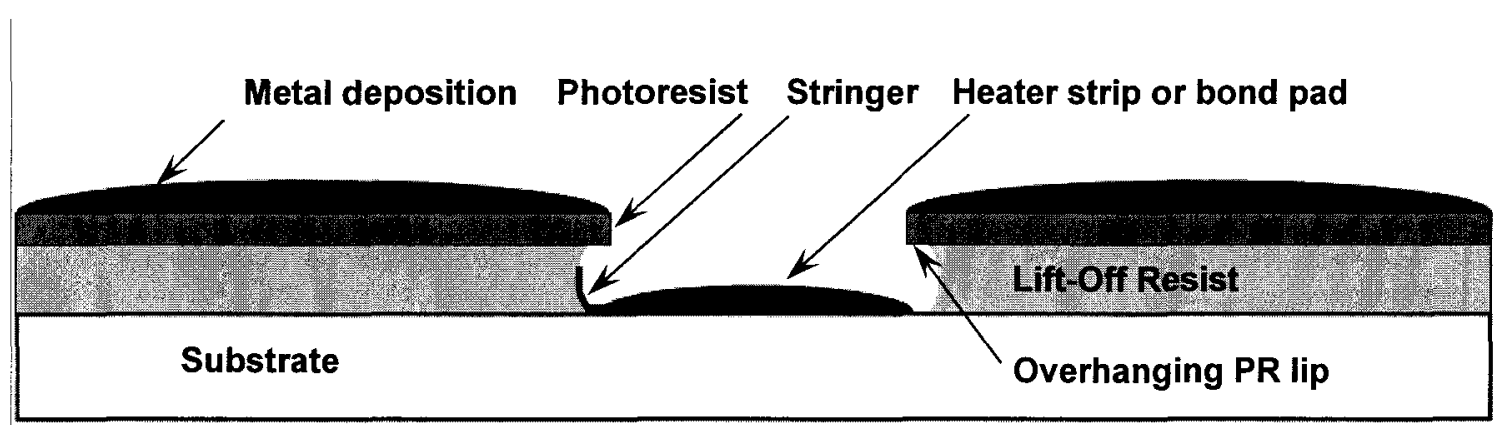

Figure 5.11. Cross-section diagram of Lift-off resist process for heater strip or bond pad.

The width of the overhanging lip is crucial to the success of the lift-off of the PR and the metal which rests on it. If there is too little overhang, the metal can coat, and therefore protect the sidewall of the lift-off resist. If there is too much overhang, the resist lip will fold over during the develop process, again preventing the LOR from acting as a sacrificial layer. A photomicrograph depicting the overhanging lip is shown in figure 5.12.

Of the two metal deposition processes discussed in section 5.3, sputter deposition is particularly prone to partial sidewall coating. After the lift-off process is complete this sidewall coating can lead to structures called "stringers". Stringers can cause electrical shorts or even effect the mechanical performance of a structure the film is deposited upon. 


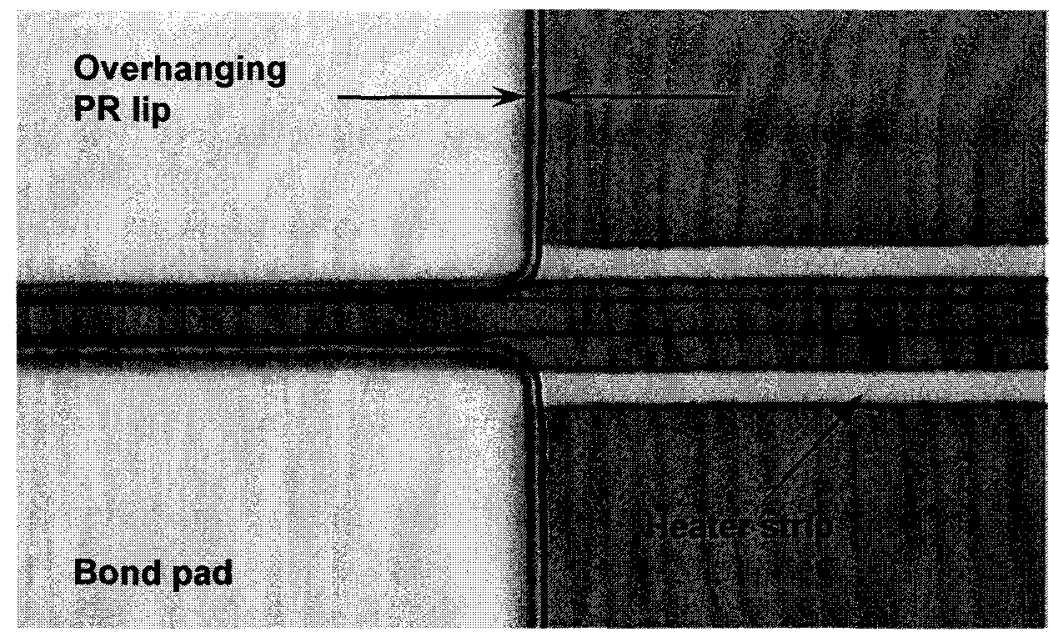

Figure 5.12. Example of overhang of PR on LOR with D1 device bond pad lithography.

Acetone can be used as the solvent for the lift-off process, but the process that was developed for this research used a dual bath in Shipley Microposit Remover 1165, at $80^{\circ} \mathrm{C}$. Microposit has a low surface tension, which allows good penetration to the LOR under the PR. This is useful in the case where the LOR film thickness is submicron, or the case where the metal on photoresist does not break up easily, such as with gold film. The dual bath allows the first bath to remove the bulk of the lift-off material, while the second bath allows for a cleaner finish to the metal edge.

To further assist in the LOR removal step, a heated ultrasonic bath was used. The vibration would further assist solvent penetration to the LOR under the PR layer. In cases where it was suspected vibration might cause damage to the deposited pattern, heating the Microposit on a hotplate alone could be used. When required, removal of the LOR film could be assisted by gentle abrasion with a cotton swab.

\subsubsection{Inspection and Metrology}

The handling techniques for small die, selection of PR, spin-on speed, exposure and develop time, and bake temperature and duration were all process parameters that 
were adjusted and balanced to produce a resist linewidth that corresponded well to the dimensions specified by the design layout. Inspection and metrology were used to measure the results of these parameter adjustments.

An important tool for inspection is the lithography microscope. It is used to ensure the immersion in MF-321 develops all the mask features into the resist. The microscope uses a green filter so that die can be inspected without exposing the PR that was masked. If some features are still not open in the resist, the die can still be placed back into the MF-321 to be developed further at a sacrifice to the overall PR thickness.

The tools used for PR metrology are the Tencor P1 profilometer for step height and surface roughness measurement and the Nanospec 3000 reflectometer for film thickness measurement. These machines are shown in figure 5.13. Linewidth (LW) metrology was accomplished with Lumenera Infinity Analyze software in combination with digital image capture from the Zeiss Axiotron microscope. The lab's JEOL JSM840 scanning electron microscope (SEM), shown in figure 5.14, was used to supplement or enhance all these measurements.

Naturally, this equipment was also used for metrology in other stages of process development and inspection. For example, the SEM was an indispensable tool to determine the success of waveguide cleaves, as well as for bridge release inspection. 


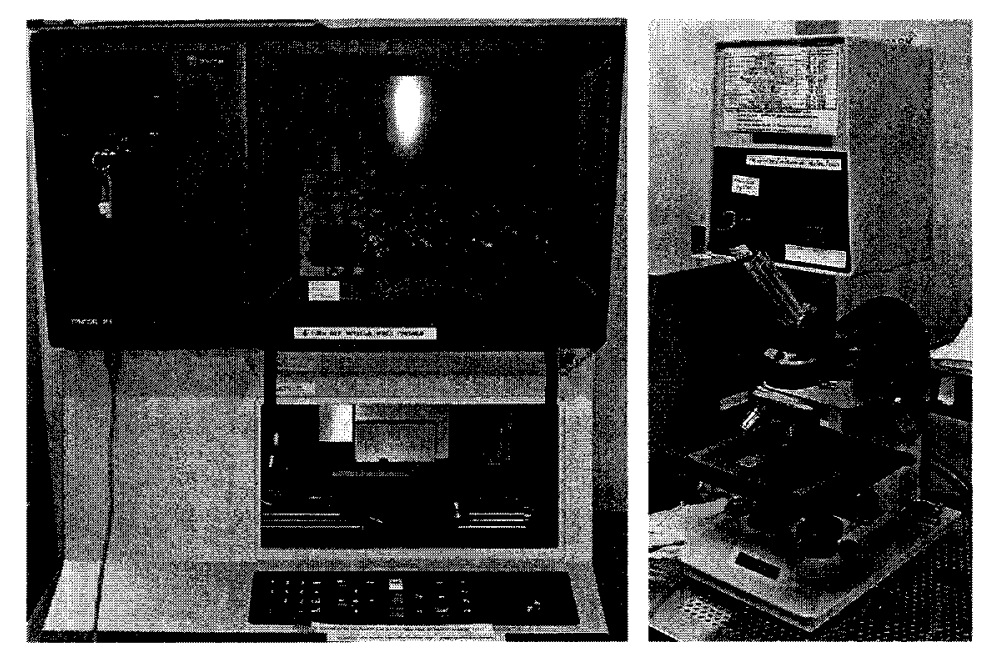

Figure 5.13. The Tencor P1 profilometer and Nanospec 3000 reflectometer.

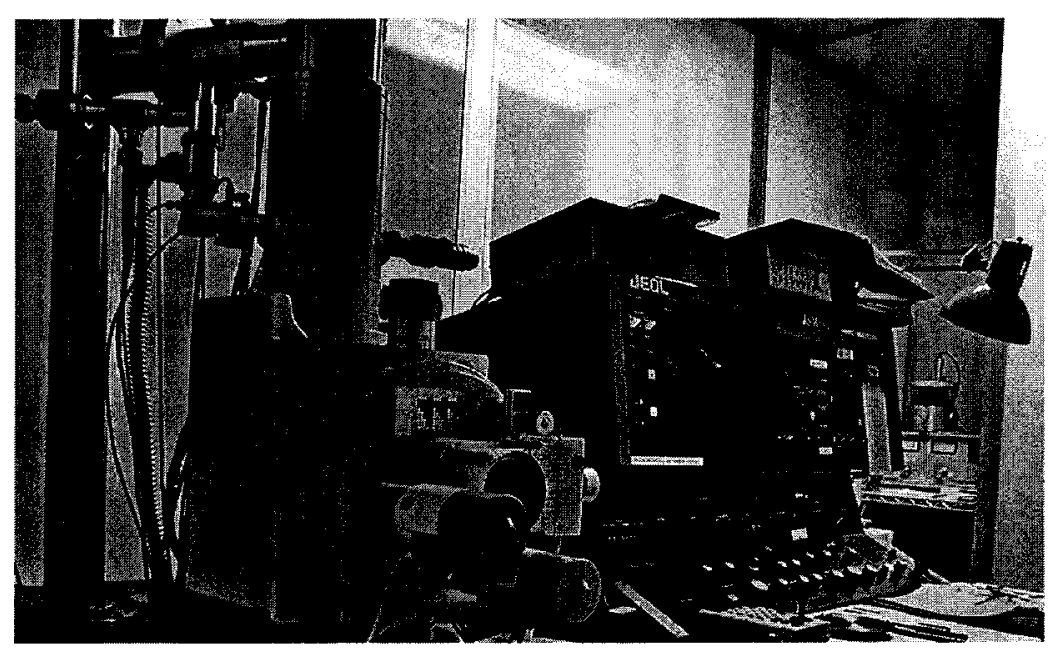

Figure 5.14. JEOL JSM-840 Scanning electron microscope.

\subsection{Dry Etch Processing}

Dry etch processing could be used for a variety of tasks, such as cleaning or etching silicon, or sharpening the edge of a PR mask. After each of the grating, waveguide and bridge structures had been lithographically patterned on the silicon surface, the next step was to dry etch the exposed silicon.

Plasma etch systems contain a reaction chamber that operates under a vacuum. 
The wafer or die is placed between parallel conducting plates in the chamber and a reactant gas, or gases, is pumped into the evacuated chamber. Next an oscillating RF field, in the power range of hundreds of watts, is applied to the plates. The field ionizes the gas, generating a plasma which interacts with the wafer. The resulting etch mechanism is a combination of the reactive chemical component of the gas and the kinetic impact of the ions on the wafer surface. The complexity of this process is in balancing pressure, gas flow, and power, to etch the target material to the desired sidewall profile while maintaining appropriate selectivity over the mask material.

The Plasmatic Systems Plasma Preen II etcher/cleaner, shown in figure 5.15, is the most simple of the MFL plasma etchers. It is a converted microwave oven, but can be thought of as a barrel reactor type of plasma etcher. The name derives from the original shape of such etchers, but the technical meaning is that purely chemical plasma etching occurs. There are no fields present to direct the reactants in the plasma towards the surface being etched.

The wafer or die is sealed within a quartz reaction chamber, which is pumped down to a vacuum of 4.0 Pa. Oxygen gas is then introduced to the chamber at a pressure of $670 \mathrm{~Pa}$. When the machine exposes the $\mathrm{O}_{2}$ gas to microwave radiation from a standard microwave oven source, the individual gas molecules are ionised. The resulting oxygen plasma in this reaction creates a temperature on the surface of the die that is in excess of $200^{\circ} \mathrm{C}$. Any organics such as $\mathrm{PR}$ are reduced to ash and removed via a vacuum pump. This plasma ashing technique is used to clean or strip PR from a wafer or die. It was also used in the process developed to release the bridge structures via sacrificial PR ashing. 


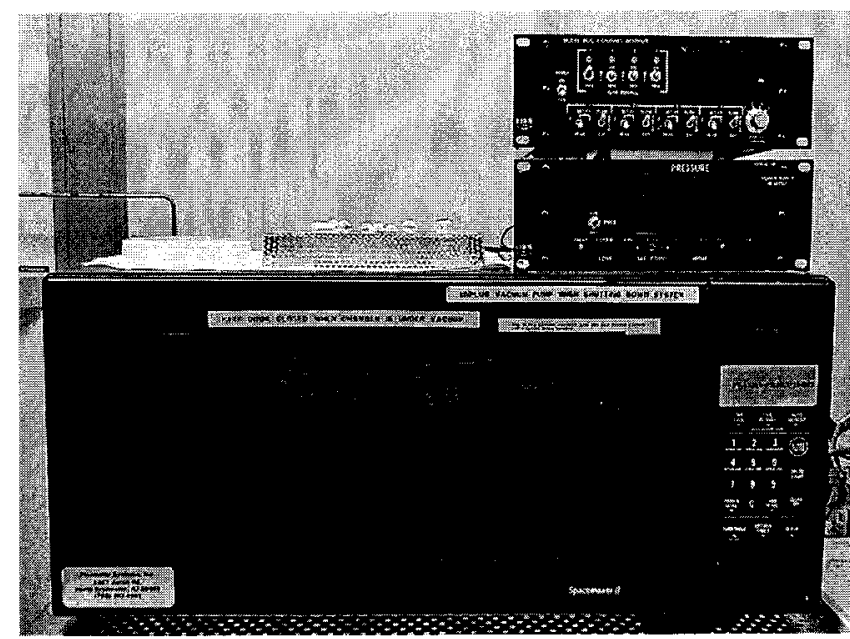

Figure 5.15. Plasmatic Systems Plasma Preen II plasma etcher/cleaner

A lower temperature etch, or descum etch, was performed with the Technics Planar Etch II parallel plate plasma etcher, shown figure 5.16. A $30 \mathrm{kHz}$ RF field ionizes and then accelerates the individual gas molecules to the substrate surface. This plasma etch system uses a combination of chemical and physical reactions, which results in a lower temperature etch at a much slower etch rate. The oxygen plasma used to ash organics from a substrate in the reaction chamber of this etcher will not strip resist from the sample. Instead the lower temperature descum etch straightens the edge of exposed PR mask for a sharper silicon etch profile. Other organics, including developer residue, are also removed from the exposed silicon substrate during the descum etch to reduce micromasking.

Typically the reaction chamber is pumped down to a base pressure of $4 \mathrm{~Pa}$, then oxygen is introduced at a pressure of $40 \mathrm{~Pa}$. The ionization power is $100 \mathrm{~W}$ to $300 \mathrm{~W}$ depending on the purpose of the etch, while etch times range from 10 seconds to 45 minutes. The Technics etcher was also configured to be used with a $\mathrm{CF}_{4} / \mathrm{O}_{2}$ plasma. This chemistry was used to etch silicon and was used in the initial bridge etch 
experiments. These various processes are detailed in their respective run sheets in appendix II.

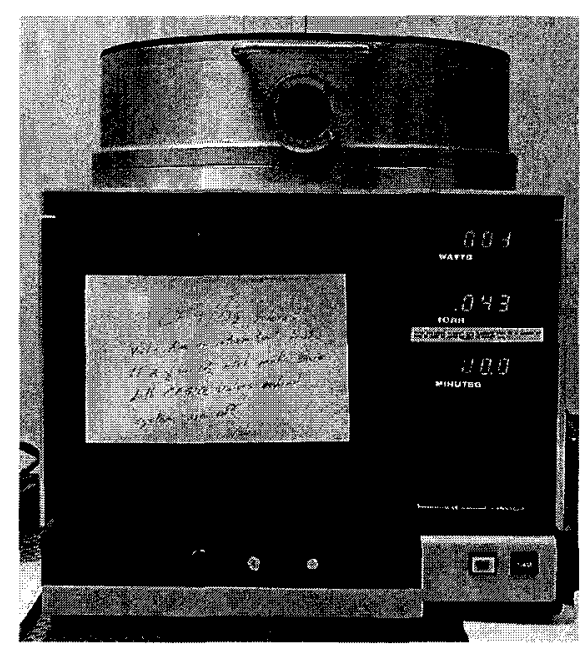

Figure 5.16. Technics Planar Etch II plasma etcher used primarily for descumming.

\subsubsection{Grating and Waveguide Etch}

The Plasma Preen and Technics etchers were used primarily for substrate preparation such as cleaning, photoresist removal, or descumming; or post-processing steps such as the bridge release. To conduct the Bragg grating, rib waveguide, and bridge etches, the advanced silicon etching capabilities of the PlasmaTherm SLR-772 Electron Cyclotron Resonance (ECR) Reactive Ion Etcher (RIE) were required.

The PlasmaTherm, hereafter referred to as the ECR, balances a variety of parameters to achieve its etch performance. The machine makes use of both microwave and RF energy in the etch process. This allows independent control of ion current (plasma density) and ion energy so that the chemical and mechanical components of the etch can be controlled and tuned separately. A magnetic field generated by solenoid coils interacts with the primary excitation source, the microwave generator, to create a highdensity plasma. The RF power source biases the wafer in order to steer ionic species in 
the plasma normal to the wafer surface, which affects the directionality of the etch. The plasma density for this process is high compared to the other plasma etch equipment - in the range of $10^{11}$ to $10^{12}$ ions $/ \mathrm{cm}^{3}$. The high plasma density allows for a lower pressure in the reaction chamber while maintaining high ion flux and etch rate. With fewer gasphase collisions there is more directionality in the etch.

The target wafer is cooled via thermal contact by He backside cooling. The lower temperature reduces the chemical component of the etch, which is a contributor to etch isotropy. Without cooling, ions continue to bombard upward-facing surfaces and etch them away. These control elements are summarised in figure 5.17 [96].

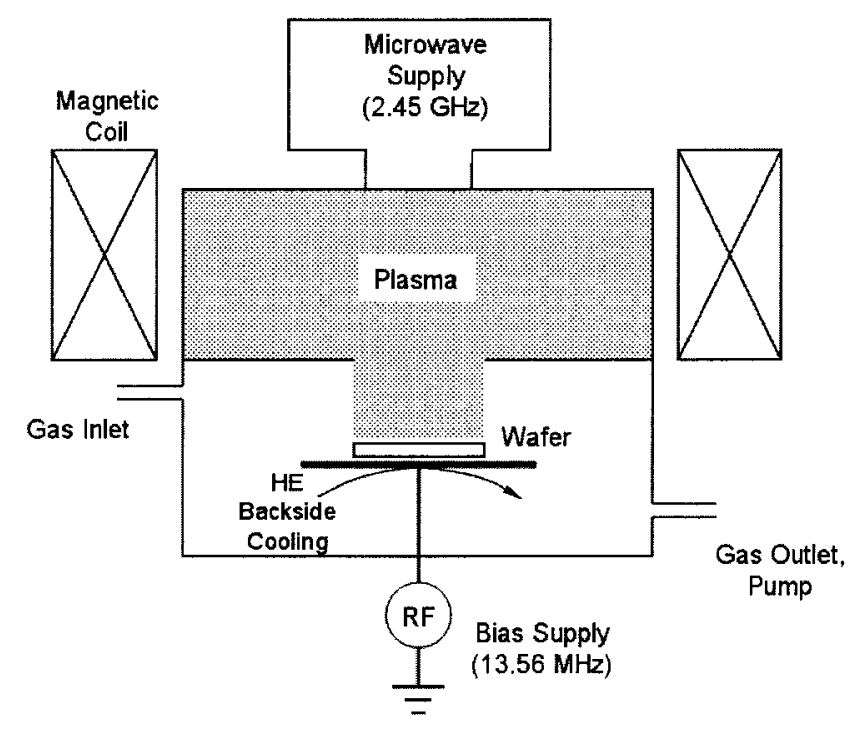

Figure 5.17. Main etch control elements of an ECR RIE [after 96].

Controlling the plasma density, ion energy, plasma chemistry, directionality and temperature affects the etch rate, anisotropy and selectivity. Developing process parameters to balance these controls results in an anisotropic etch of the silicon with good selectivity against the mask material and a smooth etch surface.

The baseline recipe for operation of the ECR, including the ECR power (microwave driven), the lower electrode power at the wafer (RF driven), gas flows, 
pressure, and the wafer temperature were developed by Professor Gary Tarr and Robert Vandusen to produce an anisotropic etch with a slightly negative undercut. The undercut prevents silicon grass from forming due to micromasking, while still maintaining a vertical sidewall etch profile. In a typical etch run the reaction chamber was evacuated to $2.4 \times 10^{-4} \mathrm{~Pa}$ and the wafer or die was cooled to $-30^{\circ} \mathrm{C}$. SF 6 was introduced at $3.65 \mathrm{sccm}$, and $\mathrm{O}_{2}$ at $2.21 \mathrm{sccm}$. The chamber was maintained at $0.8 \mathrm{~Pa}$ throughout the etch process. Forward microwave power was $310 \mathrm{~W}$ and the RF bias was $10 \mathrm{~W}$. The ECR is shown in figure 5.18 .

The smallest wafer the load lock wafer handling system of the ECR could accommodate was $100 \mathrm{~mm}$. During a typical etch run, four $7 \mathrm{~mm} \times 12 \mathrm{~mm}$ die were affixed to the centre of a $100 \mathrm{~mm}$ backing wafer, where the etch chemistry and energy conditions would be the most consistent. Either PR or double sided carbon tape was used as an adhesive. Carbon tape was preferred as it provided much better thermal coupling to the cryogenically cooled wafer, ensuring similar etch conditions that a $100 \mathrm{~mm}$ wafer alone would experience. The use of PR as an alternative backside adhesive produced a change in etch rate, anisotropy, and selectivity that were exploited during process development.

Shortly after process development on the D1 stream began, the performance of the turbo-pump used to evacuate the reaction chamber and maintain pressure during the etch became irregular. Due to instability in the vacuum performance of the ECR, there were problems with the quality and reproducibility of the Bragg grating and rib waveguide etch. Examples for both grating and waveguide etches are shown in figure 5.19 . 


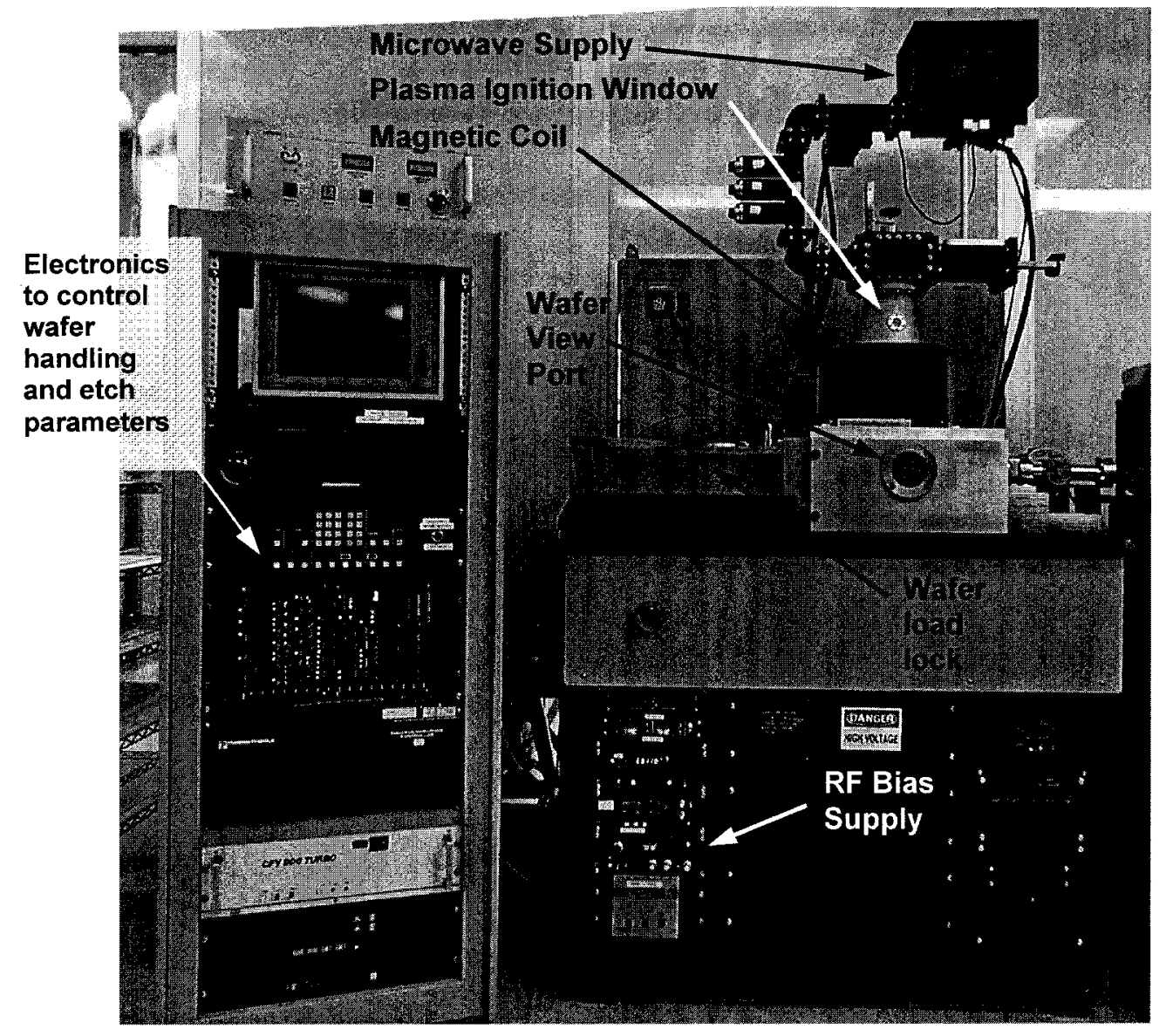

Figure 5.18. The Carleton University MFL PlasmaTherm SLR-772 ECR RIE.

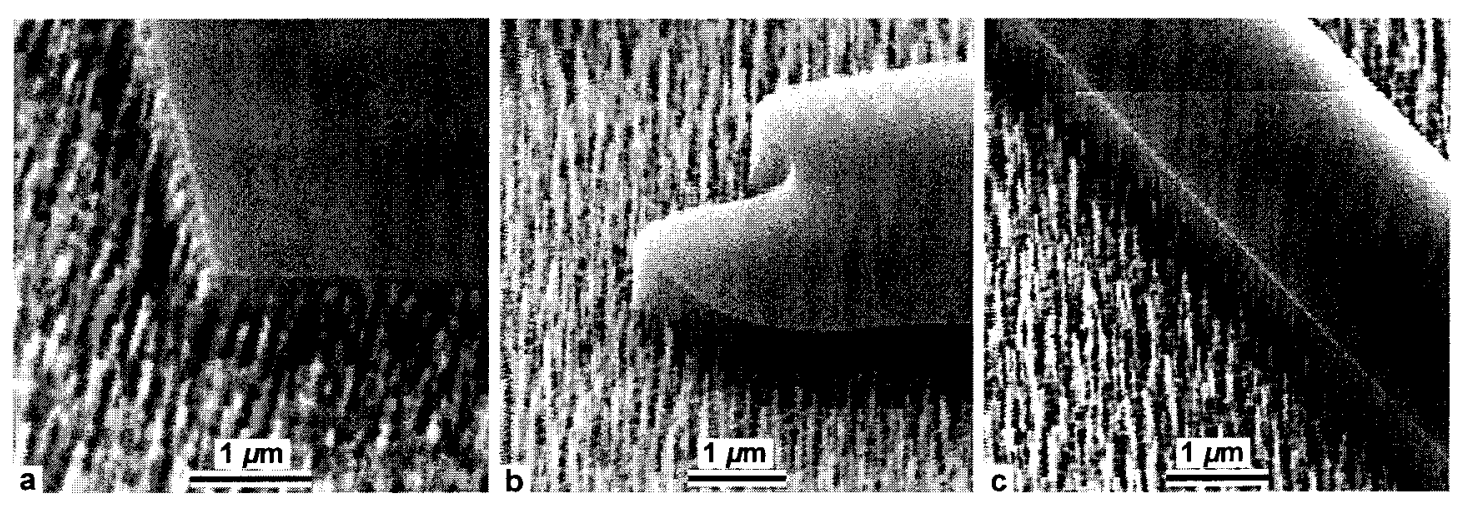

Figure 5.19. Silicon grass from the a) grating etch, and b) and c) waveguide etch, resulting from non-ideal etch parameters.

Micromasking and extreme anisotropy in the etch produced "silicon grass" on the etched surfaces. This extreme surface variability from the silicon grass could cause problems with adhesion both in lithographic and metal deposition steps. Etch rates were 
also inconsistent between die from the same process run as well as between runs. Etch rate inconsistency made it difficult to control the depth of the waveguide rib. It was also difficult to control the Bragg grating depth while maintaining a constant grating duty cycle. To compensate for this instability, further experimentation and adjustments in the baseline etch recipe were required.

When PR was used to cover the surface of the backing wafer, the chemistry of the PR changed the anisotropy of the etch. This change was enough to prevent the silicon grass from forming, while still maintaining a vertical etch profile. The rate of the silicon etch and the selectivity towards silicon also increased. While this was desirable for the waveguide etch, it also resulted in undercutting the submicron width grating teeth, leaving 10 - 20 nanometre wide cantilevers. The results from this stage of process development are shown in figure 5.20. By experimenting with the amount of surface coverage for the PR on a backing wafer, a balance was established. A $75 \mathrm{~mm}$ x $50 \mathrm{~mm}$ opening on the centre of a $100 \mathrm{~mm}$ backing wafer coated with PR was ideal.
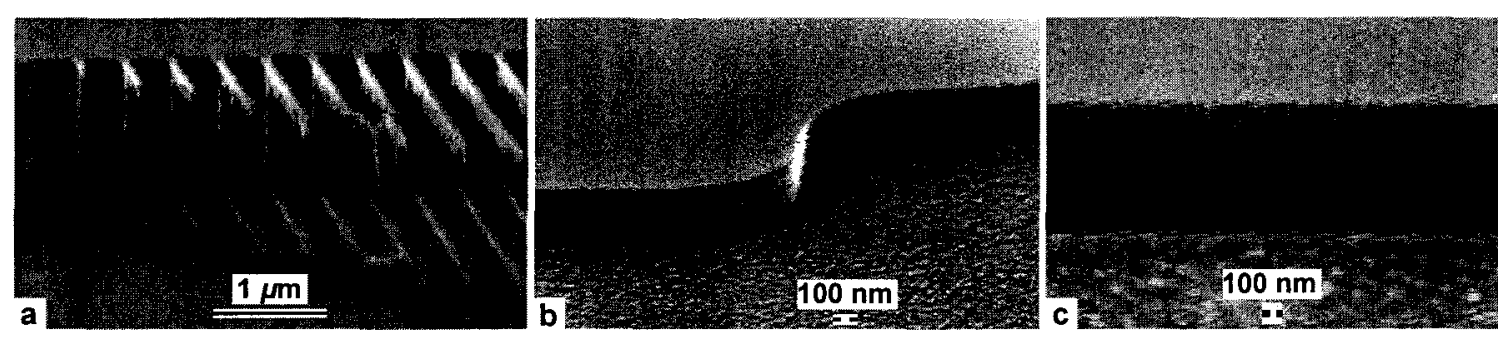

Figure 5.20. PR tuning the anisotropy of the etch removed the silicon grass b) and c), but undercut the Bragg grating a).

As experimentation and process development to address etch instability continued, it was discovered that another contributing factor in ECR unpredictability was due to the fact that when the microwave power was turned on, the plasma would not always ignite immediately. A delay of up to 10 seconds could occur, throwing the RF 
reflected power out of automated balance and allowing up to $4 \mathrm{~W}$ of RF power to be lost. This would effect how the ions were drawn to the base where the sample was etched. The problem was solved by manually fixing the RF bias to its preset state before the microwave power was turned on to ignite the plasma. Once plasma was observed through the ignition window, the RF auto-tuning controller was set to automatic to maintain the $10 \mathrm{~W}$ bias throughout the etch.

With the RF bias functioning with no reflected power, the anisotropy of the etch was balanced so that vertical sidewalls could be etched without silicon grass forming. With an operating procedure developed to counter delayed plasma ignition, along with other process refinements, the kludge of altering the etch chemistry with PR was no longer needed. The progression of process development etch results is shown in figure 5.21 .

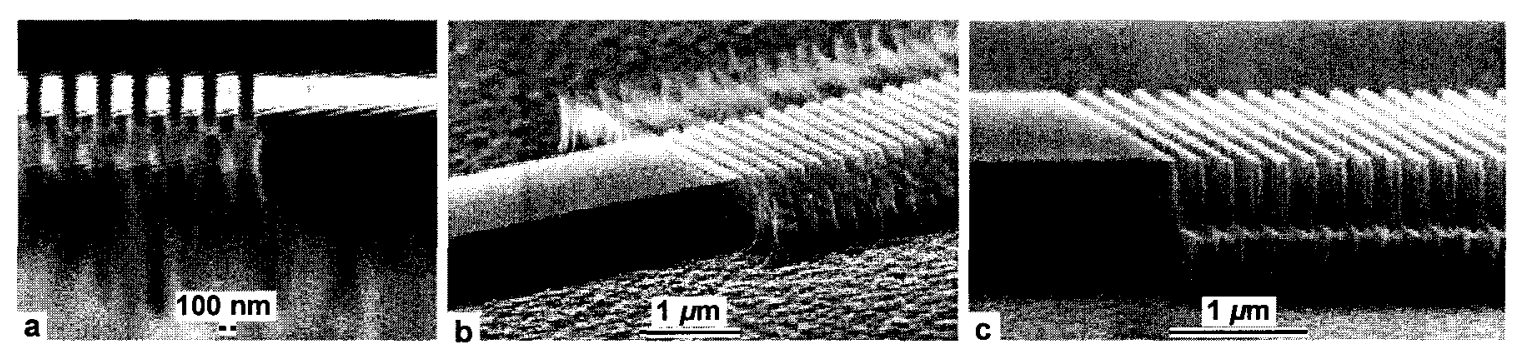

Figure 5.21. Original grating and waveguide etch on a) bare backing wafer, b) PR balanced backing wafer, and c) bare backing wafer with process improvements and ECR operation adjustments.

Although the PR wafer backing was no longer needed for the grating and waveguide etch, it was still used for the bridge etch. The bridge step required an etch through up to five microns of silicon to the BOX layer. The increased selectivity against PR and faster silicon etch rate was desirable to maintain the linewidth of the bridge mask throughout the longer etch. The silicon to mask selectivity variants were: silicon to ZEP, $7.4: 1$; silicon to PR, $10.6: 1$; silicon to PR with a PR passivated backing wafer, $26: 1$. 
The etch profile and surface roughness could now be controlled, but the etch rate, even on die in the same etch run, still varied. Experimentation determined that the high selectivity of the etch against $\mathrm{SiO}_{2}$ was such that the native oxide on the silicon surface would influence the timed silicon etch rate. A major factor influencing the thickness of native oxide present on the die would depend on its place in the process flow. An exposed silicon surface at the stage of the Bragg mask had a nominal native oxide thickness of $15 \AA$, but by the time the die had been stripped of ZEP in the PlasmaPreen and the waveguide mask had been processed, the thickness was in the range of $50 \AA$. The AME 550 ellipsometer was used to measure the presence of native oxide, as the Nanospec could not be used for film measurements of this thickness.

To characterise the influence on a waveguide etch, a 15 second buffered oxide etch (BOE) dip in buffered hydrofluoric acid (BHF) was performed minutes before insertion into the ECR. Although native oxide would begin to regrow immediately, the layer would be uniform across the four die on the same backing wafer. Table 6 summarises these results. With a pre-etch BOE dip, the difference in etch rate for die in the same etch run ranged from $0 \%$ to $4 \%$. The difference in etch rate for die that did not receive the pre-etch $\mathrm{BOE}$ dip varied from $3.2 \%$ to $32 \%$ in the same etch run. It is clear that a BOE dip before the silicon etch greatly improves etch rate consistency, so this step was added to the process flow that was developed.

With the etch process stabilised, the line width bias of the waveguide could be established. A $5 \mu \mathrm{m}$ waveguide mask line width yielded a waveguide rib width ranging from $4.7 \mu \mathrm{m}$ to $5.2 \mu \mathrm{m}$ wide. 


\begin{tabular}{|c|c|c|c|}
\hline Waveguide Etch & $\begin{array}{c}\text { Range of timed etch rates } \\
\text { over all runs }\end{array}$ & $\begin{array}{c}\text { \% difference in etch } \\
\text { rates over all runs }\end{array}$ & $\begin{array}{c}\text { \% difference range in } \\
\text { etch rates over die in } \\
\text { same run }\end{array}$ \\
\hline BOE before etch & $3600 \AA / \mathrm{min}$ to $4335 \AA / \mathrm{min}$ & $17 \%$ & $0 \%$ to $4 \%$ \\
\hline $\begin{array}{c}\text { No BOE before } \\
\text { etch }\end{array}$ & $2500 \AA / \mathrm{min}$ to $3840 \AA / \mathrm{min}$ & $35 \%$ & $3.2 \%$ to $32 \%$ \\
\hline
\end{tabular}

Table 6. Comparison of etch stability as a function of BOE pre-etch dip.

\subsubsection{Bridge Etch}

The dry etch to pattern the bridge structure was the most straightforward of the three silicon etch processes to develop. It involved a simple rectangular contact cut to the BOX layer that was $22.5 \mu \mathrm{m}$ wide and as long as the bridge. The distance between the two rectangles defined the $30 \mu \mathrm{m}$ bridge width. A $30 \mu \mathrm{m}$ wide bridge mask yielded a bridge line width ranging from $30.7 \mu \mathrm{m}$ to $32.6 \mu \mathrm{m}$.

Since the silicon etch was highly selective against oxide, the etch depth was selfpassivating. Even with a self-terminating end point, it was desirable to end the etch as soon as the BOX was exposed so that the PR edge defining the bridge width would not degrade. PR passivation of the backing wafer was used to increase PR selectivity to $26: 1$ and the silicon etch rate to $7700 \AA / \mathrm{min}$.

After a bridge etch was completed the die were not removed from the backing wafer until inspection ensured the BOX had been completely exposed. In the case of the PC and D1 stream, in which the BOX was one micron thick, the interference colour of the oxide thickness made plain that the oxide had been revealed. The green colour in figure 5.22 indicates a film thickness of $1.1 \mu \mathrm{m}$ [97]. In the case of the D2 stream, the interference colour associated with a two micron thick oxide was of such a high order as to be nearly colourless to the eye and essentially indistinguishable from the adjacent silicon under the inspection microscope. In this case the Nanospec was used to measure 
the presence of the oxide film to confirm the bridge contact cut etch had been completed.
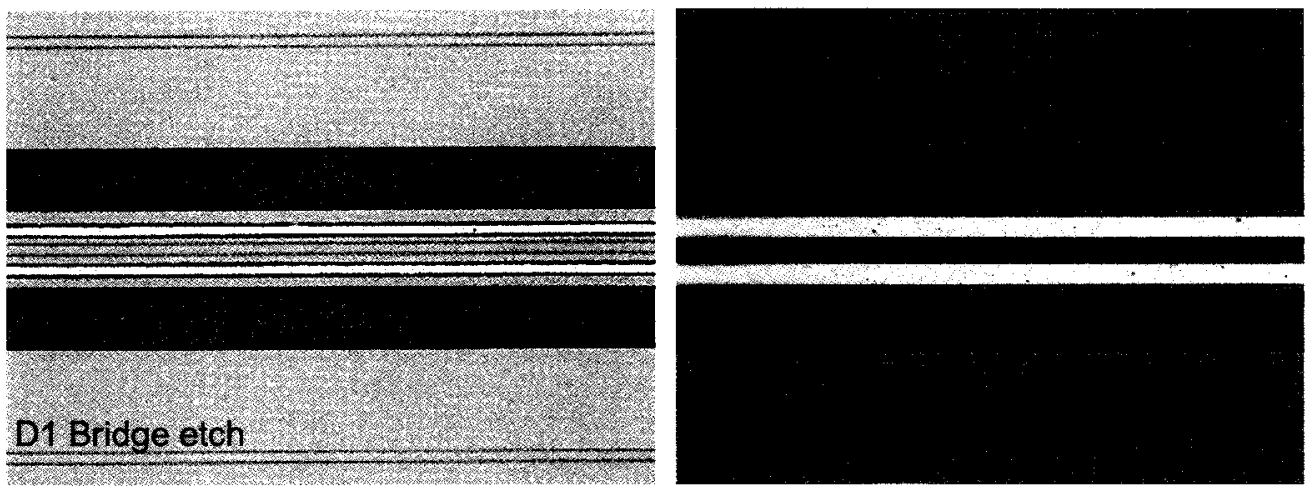

Figure 5.22. Bridge etch with one micron thick BOX for the D1 stream and two micron thick BOX for the D2 stream, each revealing their oxide interference colours.

\subsection{Metal Deposition for Heaters}

The next process step after the grating, waveguide, and bridge structures had been etched into the silicon substrate was to deposit metal heaters on either side of the rib waveguide. The heaters would provide thermal actuation to the bridges that would be surface micromachined in subsequent process steps. The Carleton University MFL has two means of depositing a thin film of metal onto a substrate, called sputtering and evaporation.

In each case the lithographic LOR technique described in section 5.1.4 was used to process what would become the stencil for the heater strips. Minor differences in lithographic considerations will be dealt with in the respective sections of each deposition technique. Deposition runs in either machine were conducted on six die at a time.

Film deposition systems can operate on either a chemical vapour deposition (CVD) or physical vapour deposition (PVD) process. Sputtering and evaporation are both PVD processes in which a target of elemental metal (or alloy) is reduced to a low pressure gas phase so that the atoms can condense on the substrate surface. This takes 
place in an evacuated reaction chamber. In the case of sputtering, the target is bombarded by energized ions from a plasma in a process that has many similarities to the RIE process. The evaporation process heats the target material until it vaporises so that the atoms are free to move through the chamber and recrystallise on the substrate surface.

Once the lift-off process described in section 5.1.4 patterned the heater strips, their dimensions were measured via profilometry and LW metrology. Next a four point probe was used to take voltage measurements on a section of the metal so that the film's sheet resistance and resistivity could be calculated. With these values and the heater dimensions, the resistance of the heater strips could be calculated and eventually compared to experimental results.

\subsubsection{Sputtering}

MFL's MRC 8620 sputtering system, shown in figure 5.23, was used for thin film deposition during the D1 process development. Non-reactive and with a comparatively heavy atomic mass, Ar gas is used to form the ionized plasma. These ions physically sputter metal off the target so it can recrystallise or condense on the substrate surface.

The sputter process involves three steps to ensure good adhesion of the film to its substrate. Once the sample has been inserted into the reaction chamber, it is evacuated to a pressure of $2.0 \times 10^{-4} \mathrm{~Pa}$. The flow of $\mathrm{Ar}$ gas into the chamber raises the pressure to $19 \mathrm{~Pa}$.

The first step, called the sputter etch (SE), prepares the substrate for the material deposition. In addition to the descum step that has been performed on the die before insertion into the reaction chamber, the SE uses the same Ar ion plasma that sputters metal off the target to clean the substrate surface. $80 \mathrm{~W}$ of RF power is used for this step. 
A macroscopic analogy for the SE step would be sandblasting a surface to clean it.
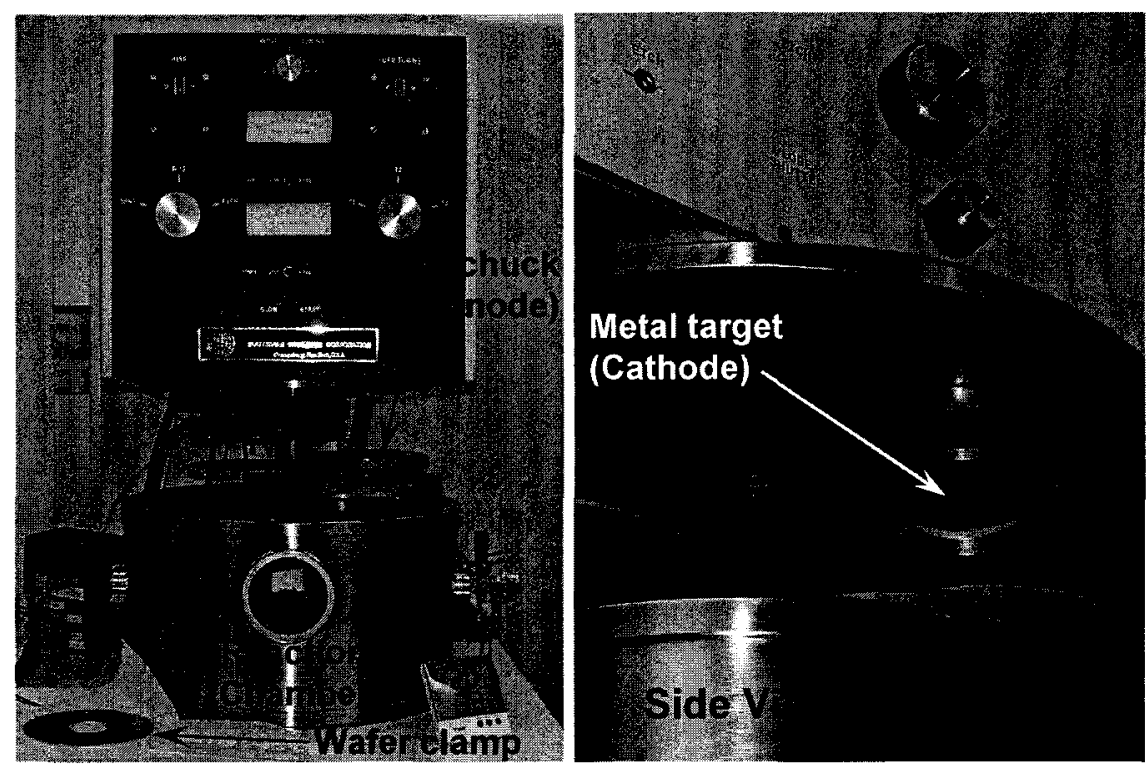

Figure 5.23. The MRC 8620 sputtering system

The next step involves shielding the wafer substrate with a shutter so that a presputter (PS) step can be performed. This step is similar to the actual sputter process, but the goal is to clean the surface of the target before the actual target material can be deposited on the substrate.

The final step is the actual sputter deposition. The wafer substrate is brought into direct exposure to the target and the sputter process begins. $75 \mathrm{~W}$ of $\mathrm{DC}$ power is used for this step. Since the target is conductive, and forms the upper portion of the plate (or cathode), an RF source is unnecessary. Were the target to be non-conductive, RF power would be used here as well.

A chiller is used to hold the wafer chuck at a temperature of $3-5^{\circ} \mathrm{C}$. Since the die is not thermally coupled to the chuck, the plasma will eventually heat the substrate itself. To help protect the LOR from thermal damage the sputter process was performed in equal on/off intervals of five minutes to allow the substrate to cool.. 
$\mathrm{NiCr}$ adheres to a silicon substrate without an adhesion layer, while the $\mathrm{NiCr}$ itself formed the adhesion layer for the gold bond pads. The 3 inch diameter $\mathrm{NiCr}$ and $\mathrm{Au}$ disk targets were of a purity of $99.99+\%$ and $99.999 \%$ respectively. An alloy of $80 \%$ nickel and $20 \%$ chromium $(\mathrm{NiCr}$ ) was chosen for its excellent electrical characteristics as a resistive heating element (high temperature endurance and high sheet resistance). The general process flow and parameters for $\mathrm{NiCr}$ on MFL's sputterer was mature so further process development was unnecessary for deposition of the $\mathrm{NiCr}$ film.

NiCr's hardness and resistance to high temperature made the material impossible to wire bond to for the packaging post-process. Wire bonding will be formally covered in section 5.6, but it is possible to attempt a combination of bond power and temperature such that enough gold bond wire is left behind to form the foundation for the actual wire bond. $\mathrm{NiCr}$, however, was so refractory as to be beyond this technique, leaving only an oval outline of the bond wire gold in the shape of the bonder wedge, as shown in figure 5.24. This necessitated the generation of a bond pad mask and a second liftoff/deposition step. Gold was chosen over the other common bond pad material, aluminum. Aluminum would not withstand the HF bridge release step. The final processed heater and bond pad structures are shown in figure 5.25.

Lift-off of the sacrificial LOR was accomplished as outlined in section 5.1.3. An ultrasonic bath was used for both the $\mathrm{NiCr}$ and gold layers. $\mathrm{NiCr}$ is relatively brittle so exposure to the ultrasonic bath was minimized. The "strip" shape of the heater mask also gave the solvent wide access to the LOR, and the lift off process took 20 minutes. 


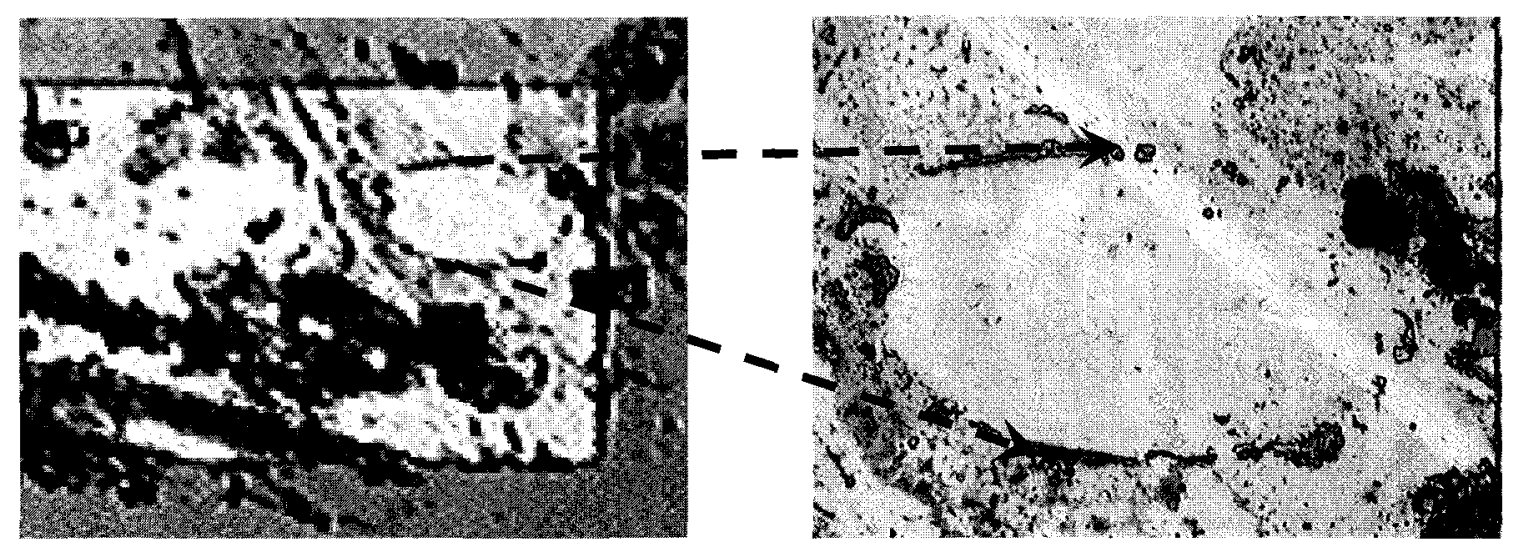

Figure 5.24. Oval outline of bond wedge in remaining gold bond wire on the $\mathrm{NiCr}$ surface.
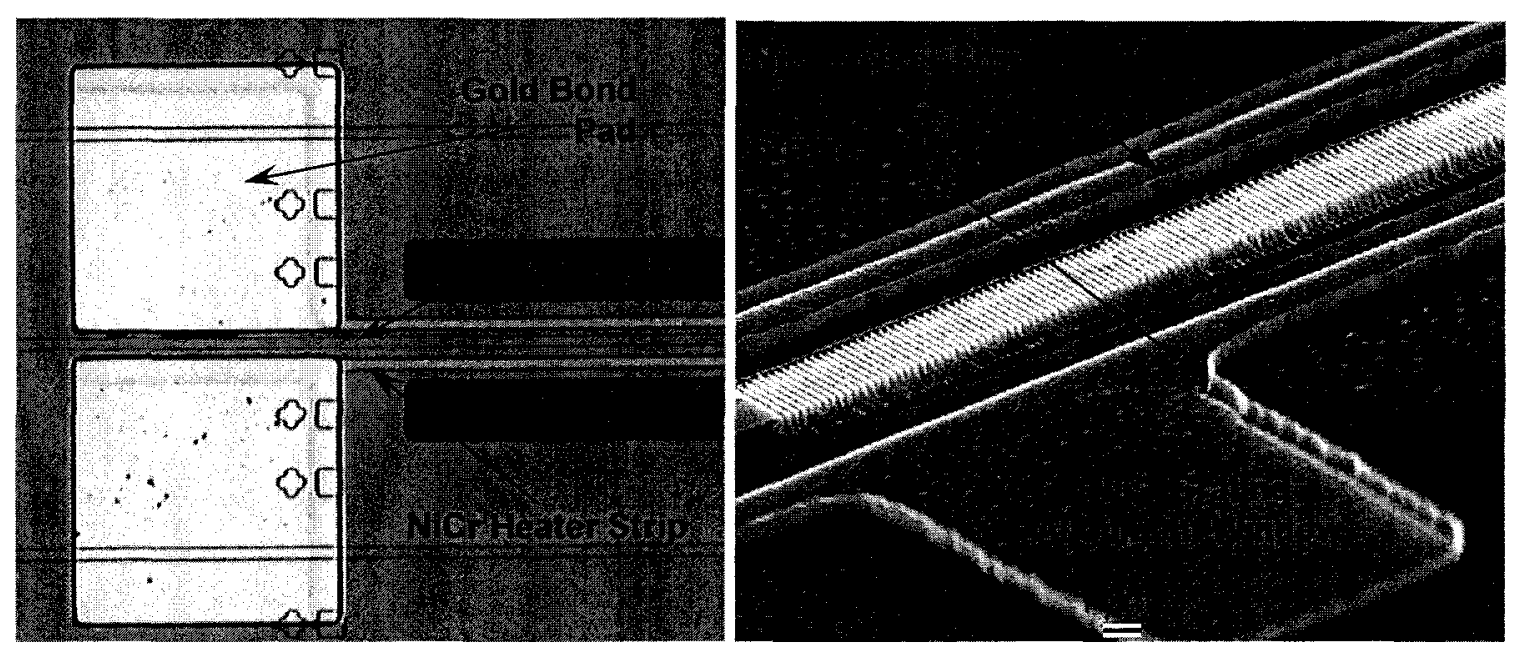

Figure 5.25. NiCr heater strip and gold bond pad via sputter deposition

Lift-off for the gold bond pads was more challenging. The only access to the sacrificial LOR was through the $100 \mu \mathrm{m}$ square pads. Access to the LOR along the edges of the die was limited due to the mechanical edge bead removal that had taken place in lithography. Removal of the LOR, and the PR and metal on top of it, took in excess of 2 hours - even while subject to the ultrasonic bath. In one case, after four hours, the stencil was gently brushed off with a cotton swab. This was clearly a mask design issue that would have to be dealt with in the D2 stream.

Step height measurement of heaters that were used in device characterisation was 
$300 \mathrm{~nm}$. The critical dimension bias of the lift off process was such that a $5 \mu \mathrm{m}$ mask line width yielded a $4.3 \mu \mathrm{m}$ wide heater. The heater thickness and the Four Point Probe measurement of the sheet resistance yielded a resistivity of $196 \times 10^{-8} \Omega \mathrm{m}$. This compares to a handbook value of $110 \times 10^{-8} \Omega \mathrm{m}$ [90]. The measurement was made in the interest of thoroughness and to gauge the properties of the metal that had been deposited as a sanity test.

\subsubsection{Evaporation}

Once fabrication of the D1 device had been completed, results from the characterization of that device were used to make improvements in the D2 device stream. One factor that had to be taken into account when developing the D2 heater design for the new process flow was that the MRC 8620 sputtering system was no longer available for use. Another factor was the problems relating to the $\mathrm{NiCr}$ cracking and delaminating (which are discussed in detail in section 5.3.3).

It was decided that gold would be used as a heating element in the D2 design. Gold's greater ductility and an increase in heater width to 7.5 microns would help reduce strip fracture. An increase in film thickness to one micron would also reduce stress in the film. Due to the much lower resistivity of gold, and the increased cross-sectional area of the heater strip, the heater resistance would also be greatly reduced. One benefit of switching to a gold heater was that bond pads would be unnecessary, and the overall exposure time to ultrasonic lift-off would be reduced.

The MFL uses the Balzers BA510 evaporation system shown in figure 5.26. In its current configuration, the source material is in pellet form, and is contained in a graphite crucible. An e-beam heater is used to vaporise the metal so that the atoms can move 
through the evacuated chamber and condense on the substrate surface.

The evaporation process begins by evacuating the reaction chamber; ideally to a pressure of $6.0 \times 10^{-5} \mathrm{~Pa}$. The wafer substrate is shielded with a shutter and the electron gun moves the e-beam in an $\mathrm{x}$ and $\mathrm{y}$ sweep pattern over the pellets in the crucible. When the pellets are in a molten state and the appropriate process conditions have been attained, the shutter is removed and metal deposition begins. A piezoelectric crystal is exposed to the same flux of metal vapour, and from this a deposition rate is measured. Specific process parameters are documented in run sheets in appendix III.

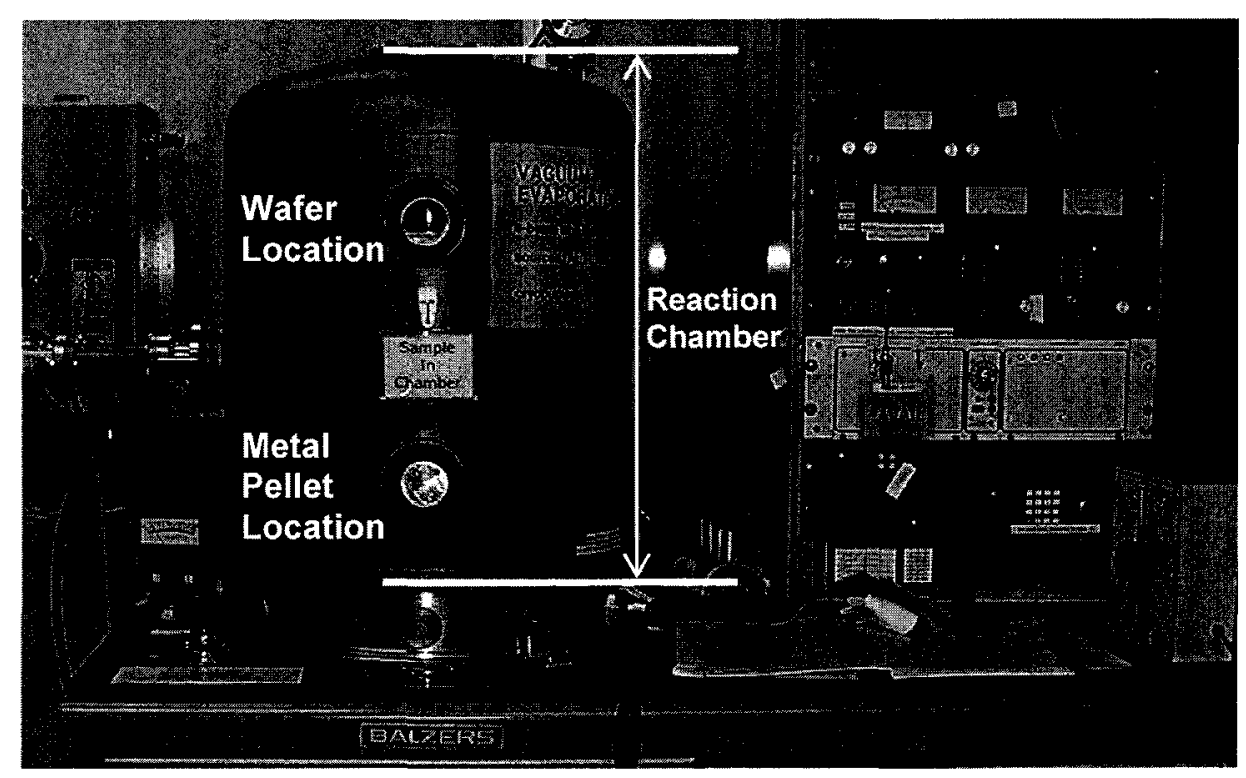

Figure 5.26. The Balzers BA510 evaporation system

Gold does not adhere well to the native oxide that is present on the silicon substrate. Therefore an initial adhesion layer must be evaporated before the gold film is deposited. Titanium and chromium are both metals that adhere well to oxide, and that gold will adhere well to. A process for gold, titanium and chromium evaporation had already been established for the Balzers and these process parameters were supplemented with information from a thin film handbook [98]. 

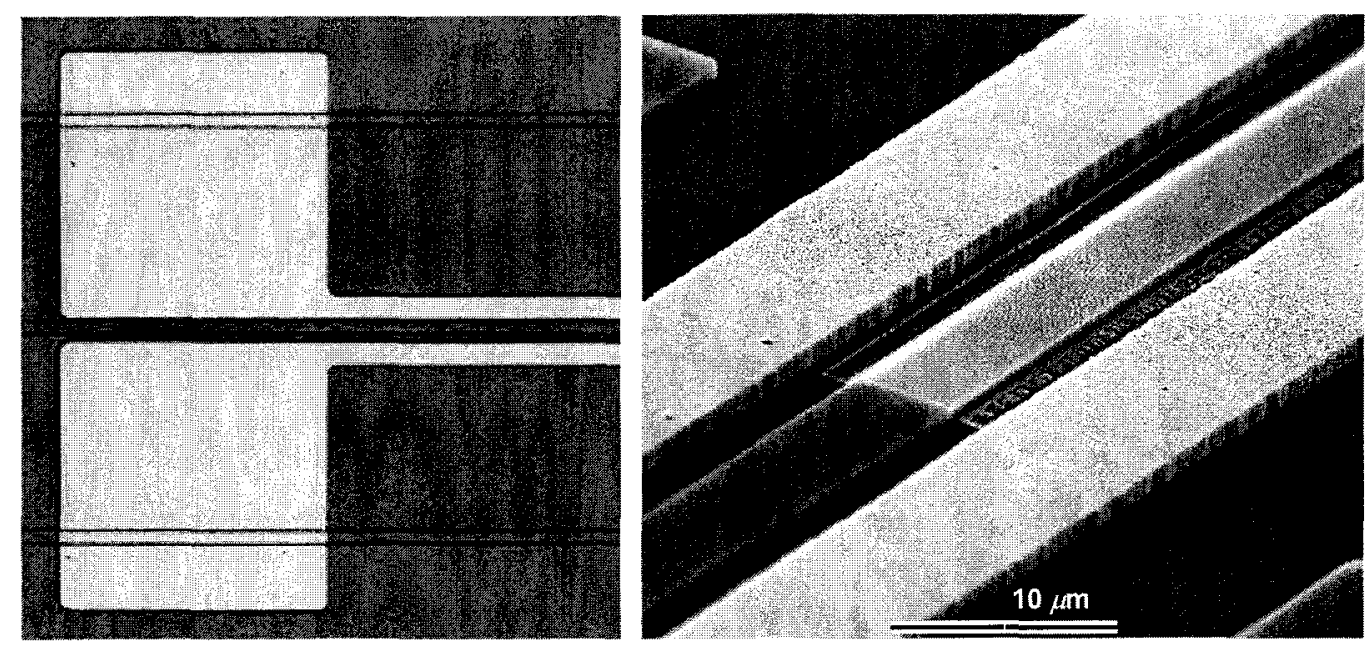

Figure 5.27. Gold heater strips and bond pads via evaporation deposition

The first process development run included a titanium adhesion layer of $260 \AA$, and a gold layer of $10,000 \AA$, evaporated under a vacuum of $2.1 \times 10^{-5} \mathrm{~Pa}$. The heater thickness and the Four Point Probe measurement of the sheet resistance yielded a gold resistivity of $2.69 \times 10^{-8} \Omega \mathrm{m}$. This compares to a handbook value of $2.24 \times 10^{-8} \Omega \mathrm{m}$ [91]. The first run using chromium included a chromium adhesion layer of $466 \AA$, and a gold layer of $10,000 \AA$, evaporated under a vacuum of $2.7 \times 10^{-5} \mathrm{~Pa}$. The heater thickness and the Four Point Probe measurement of the sheet resistance yielded a gold resistivity of $1.96 \times 10^{-8} \Omega \mathrm{m}$. The process run that generated the heaters used in lab experimentation included a titanium adhesion layer of $256 \AA$, and a gold layer of $9500 \AA$, evaporated under a vacuum of $4.0 \times 10^{-5} \mathrm{~Pa}$. The subsequent lift-off process yielded integrated heaters that were $7.9 \mu \mathrm{m}$ wide.

\subsubsection{Adhesion}

There are different methods to test the adhesion of a deposited thin film, both qualitative and quantitative, that include tape, scratch and abrasion techniques. For the purpose of testing adhesion with the thin film heaters, a simple qualitative tape method 
was used. A strip of Scotch brand tape was placed over the heater strips and smoothed over the surface to ensure the tape adhesive conformally covered both the substrate and the thin film. The tape film was then slowly and steadily pulled off at a $45^{\circ}$ angle. This test was severe considering yield was not the primary objective of the process development, but it would provide an good idea of the adhesion success.

Test heaters were deposited on bare silicon wafers, without other device structures such as waveguides or bridges, for both D1 and D2 devices. Adhesion, electrical and packaging tests were run on these heaters. HF resiliency tests were also conducted on the $\mathrm{NiCr}$ and Gold test heaters before the device die went through the full process run.

\section{Adhesion for D1 heaters}

In the case of the D1 process development, $100 \%$ of the heaters remained in place after the tape test. Immersion in concentrated HF at room temperature for a period of 15 minutes approximated the conditions of the bridge release process. HF concentration for the bridge release etch was $49 \%$ unless otherwise specified. All the heaters remained in place, indicating that a full process run could be completed, including the bridge release in $\mathrm{HF}$.

After completion of the bridge release process, all bond pads held firmly to the silicon substrate. Where the bond pads separated, it was the silicon itself that separated from the buried oxide, as shown in figure 5.28. Mechanical rupture of the silicon was likely the cause of this outcome, and not lack of $\mathrm{NiCr}$ adhesion. This effect is investigated in section 5.5 .3 on structural release shock and etch profile.

The five micron wide heater strips, on the other hand, fractured without exception. This usually occurred in the middle; and the strips, still anchored by the bond 
pad, would curl back as shown in figure 5.29. As $\mathrm{NiCr}$ is a comparatively hard and brittle material, it is likely that the extreme length of time in the ultrasonic bath cracked the heater strips and in combination with the mechanical shock of the bridge release, caused the strips to separate and curl back. When design began on the D2 device stream, large openings were placed in strategic locations on the bond pad mask. This would give the solvent much better access to the LOR and greatly reduce the time in the ultrasonic bath, should the bond pad deposition step be required.
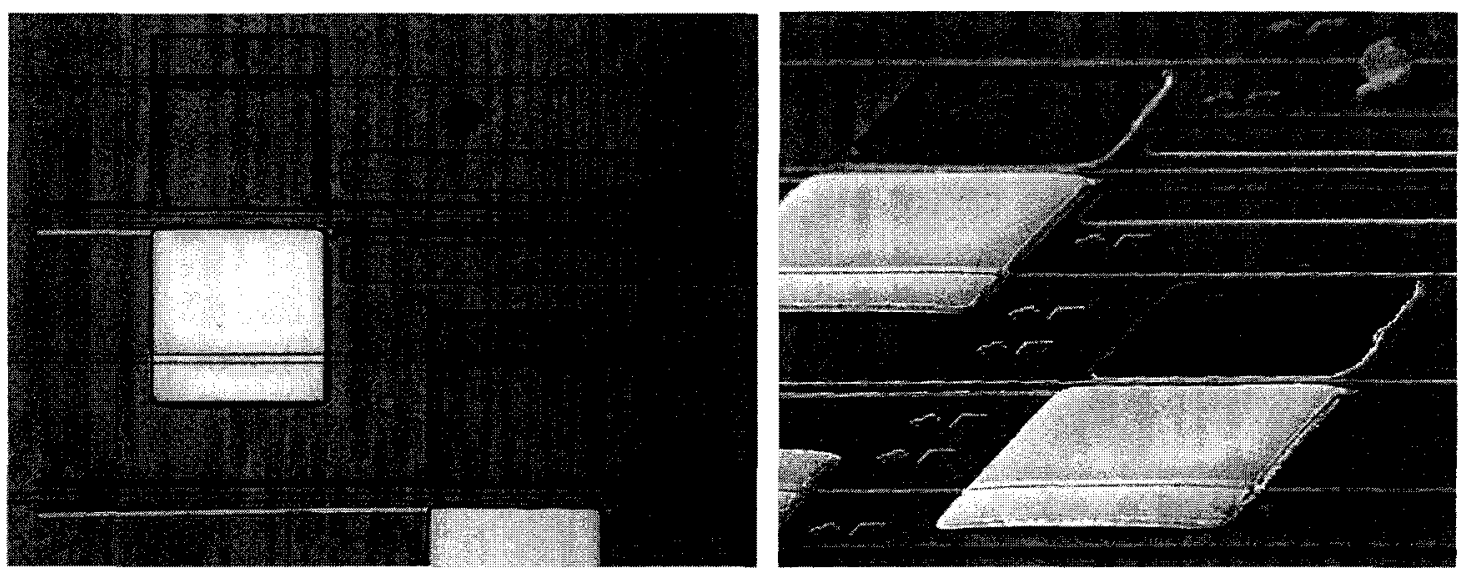

Figure 5.28. The HF bridge release on D1 die caused silicon under the bond pads to rupture, allowing the bond pads on the device layer to break away.
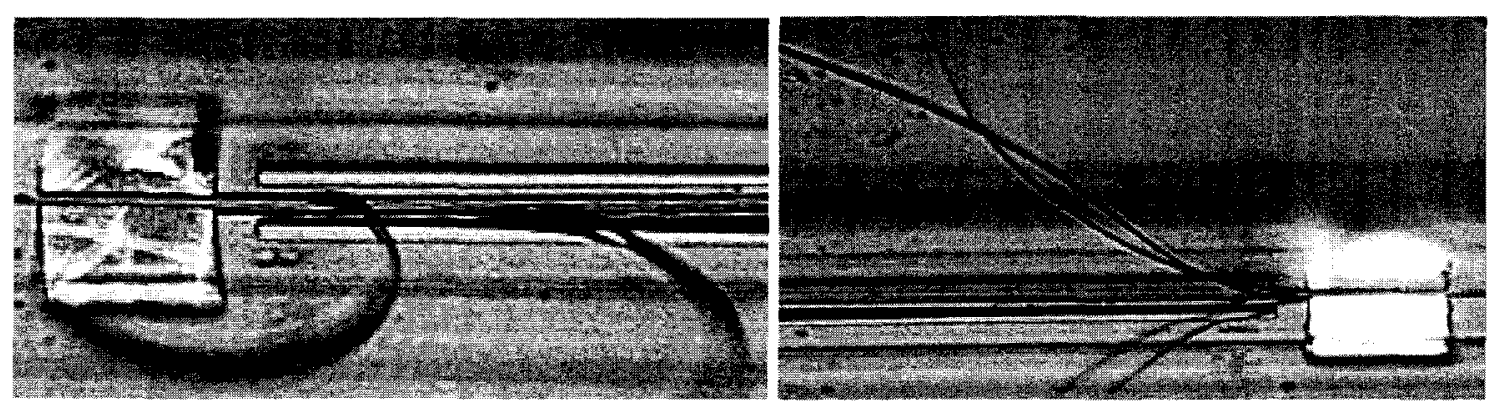

Figure 5.29. Fragmented heater strips delaminate, with bond pads remaining in place on D1 devices.

\section{Adhesion for D2 heaters}

D2 heater process development began with the evaporation of test $\mathrm{Ti} / \mathrm{Au}$ heater 
strips on a bare silicon wafer. Die from this trial were labelled D2-HT1 to D2-HT6. $100 \%$ of the gold heaters remained in place after the tape test and $100 \%$ of the heaters also remained in place after a 15 minute immersion in concentrated $49 \% \mathrm{HF}$. The tape test was also conducted after the HF immersion test. With this aggressive test 17 heater strips of all lengths, out of 30 on a die, remained.

In the next experimental run, heaters were processed on die that went through the complete process flow. The process parameters for these heaters were the same as those of the D2-HT run. After completion of the bridge release process in HF the gold heaters remained intact, unlike the $\mathrm{NiCr}$ heater strips, but the entire structure would generally delaminate as shown in figure 5.30. The heaters that did remain were the shorter $500 \mu \mathrm{m}$ and $1000 \mu \mathrm{m}$ strips, and these were poorly adhered as shown in figure 5.31. Physical contact from probing would dislodge the heaters and the wirebonder wedge would pull them off the substrate.

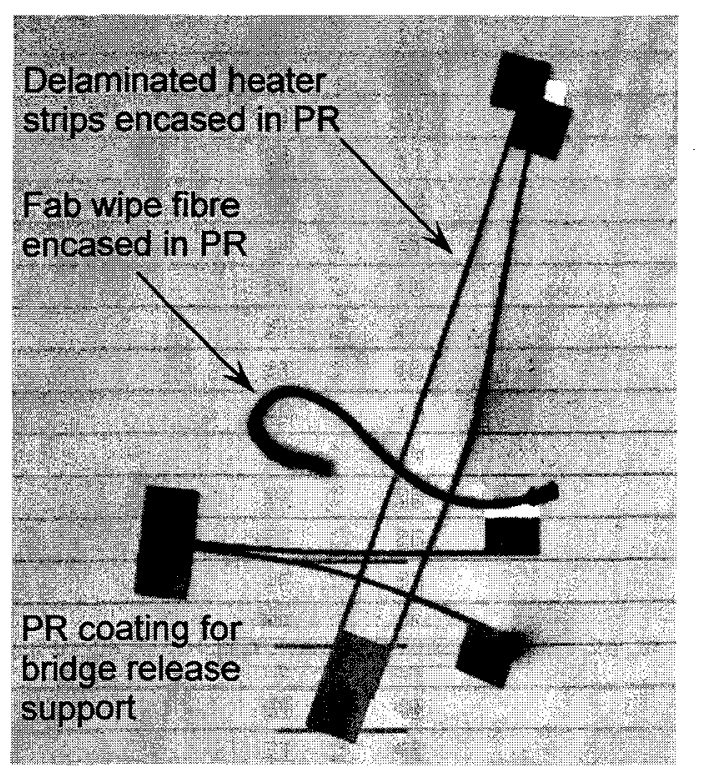

Figure 5.30. Delamination of heater strips with heaters remaining intact on D2-4 die. Infiltration replacement $\mathrm{PR}$ for bridge release support is still in place $(\mathrm{FOV}=1.1 \mathrm{~mm}$ ). 

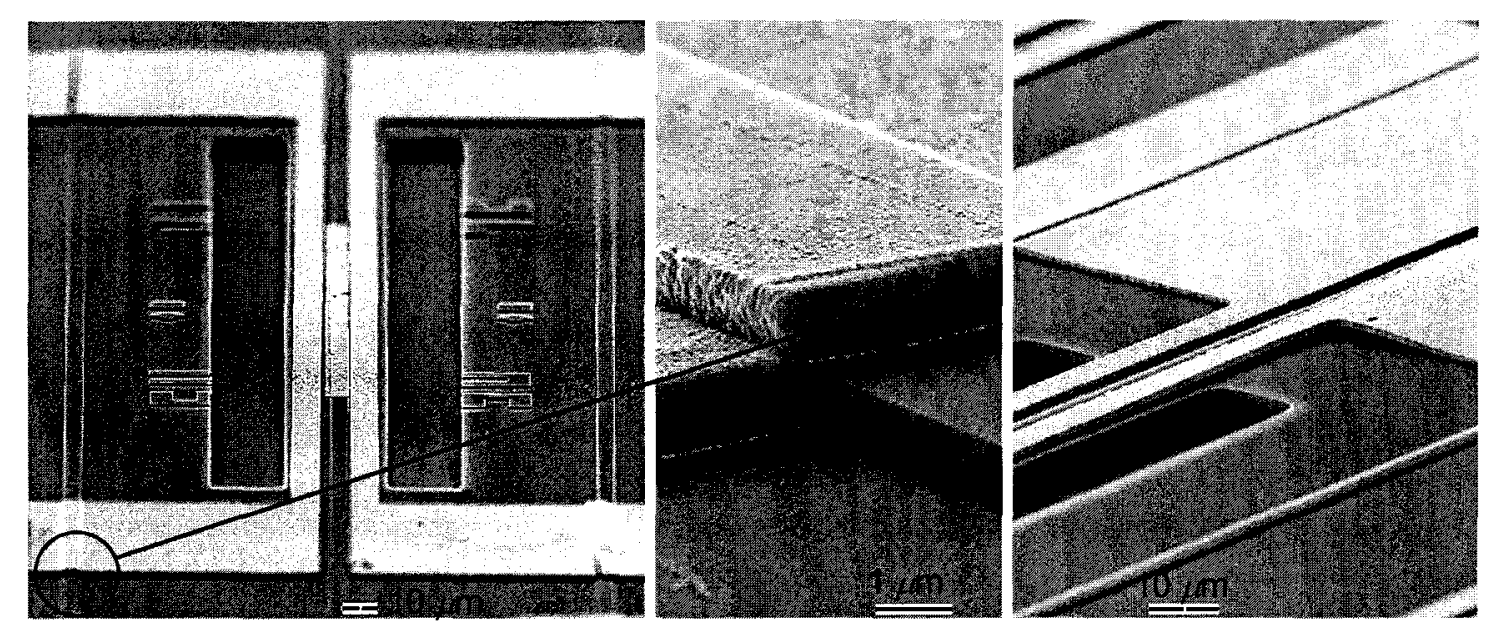

Figure 5.31. Shorter heater strips survived the bridge release process with poor adhesion and were dislodged from the force of a probe tip or wire bonder. This bridge from D2-8 is $500 \mu \mathrm{m}$ long.

Since these heaters delaminated, while the D2-HT heaters remained adhered, it was likely that the mechanical shock related to the bridge release still had an important role in heater delamination. This was combined with the observation that while the evaporated gold bond pads along with the heater strips did not adhere to the substrate, the sputter deposited $\mathrm{NiCr}$ bond pads did. This gave some indication that the condition of the substrate/adhesion layer interface also played a role in delamination of the D2 heaters during the bridge release post-process.

The etch rate of bare titanium in concentrated HF is known to be greater than $10 \mathrm{k} \AA / \mathrm{min}$ [99]. With a titanium adhesion layer thickness of $260 \AA$ it was possible that the HF could penetrate under the gold to lift the heater strips off. The chromium etch rate in HF is slow to zero [100] so it was used as the adhesion layer for the next experimental run. The adhesion strength of $\mathrm{Cr}$ has been shown to increase sharply for an increase in film thickness from $450 \AA$ to $500 \AA$ [98]. The thickness of the adhesion layer was increased to $466 \AA$. Adhesion is also known to increase over time, with the greatest increase in adhesion strength for $\mathrm{Cr}$ occurring over the first 100 hours after deposition 
[98]. Using these process changes and waiting 100 hours before executing the bridge release process, these heaters fared no better than the results described for the previous $\mathrm{Ti}$ based adhesion process.

Another possible interface factor contributing to delamination of the gold heaters was the same native oxide that interfered with the true silicon etch rate in the ECR. Elipsometry measurements of the native oxide thickness just before the metal deposition step were nominally $50 \AA$. Measurements taken on the die near the start of the process run, where only the Bragg grating mask was present, yielded a typical oxide thickness of $15 \AA$. The added oxide thickness at the heater deposition step was due largely to the oxygen plasma in the Plasma Preen, operating at an excess of $200^{\circ} \mathrm{C}$, which was used for both dehydration and PR strip in the process steps leading to heater evaporation. If the added thickness of native oxide was enough for the HF to penetrate the oxide/adhesion layer interface, this would allow the heaters to lift off the substrate [114].

A 15 second BOE dip was conducted on six die that had been processed past the heater lithography step for the purpose of minimizing the native oxide thickness at the time of evaporation. $5 \AA$ of native oxide would regrow over the 30 minute evaporator load preparation time. An adhesion layer of $256 \AA \mathrm{Ti}$ and Au layer of $9500 \AA$ were used for this experiment. The results from $\mathrm{Cr}$ adhesion layer trials were no better than heaters processed with the $\mathrm{Ti}$ adhesion layer. In the interest of varying as little as possible in each step of the process development, the initial process elements from D2-HT were restored. The resulting heaters evaporated on die that were conditioned with the BOE dip still delaminated during the HF bridge release as previous samples had.

D1 and D2 heaters were processed similarly, including the descum step just prior 
to insertion into the deposition reaction chamber. A significant process difference was the pre-sputter etch clean that the substrate received for the NiCr sputtered D1 heaters. The $\mathrm{Ti}$ and $\mathrm{Cr}$ adhesion layers evaporated for the gold D2 heaters received no equivalent etch cleaning step in the Balzers evaporation system. A one minute descum step is required to clean and prepare a silicon substrate for sputter or evaporation deposition. It was discovered during the simultaneous ECR etch and bridge release process development that the Technics plasma etcher was redepositing polymers on the substrate surface during the descum step. ECR experimental trials revealed that a one minute descum caused micromasking, and the polymer flakes are easily visible in figure 5.32 where an extended 60 minute sacrificial PR etch for a waveguide bridge release was conducted. These flakes may have provided enough surface contamination that the gold heaters would not adhere to the substrate after the bridge release; contamination that would not be present on the pre-sputter etch cleaned substrate of the D1 stream heaters.

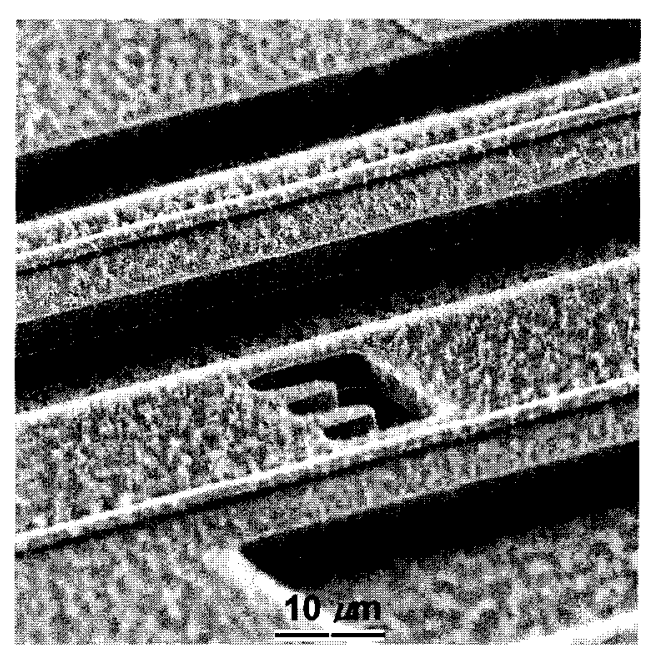

Figure 5.32. Example of redeposition of polymer flakes during a 60 minute sacrificial PR etch for a waveguide bridge release in the Technics plasma etcher.

Sputtered heaters did not suffer from adhesion as much as they did the brittleness of the NiCr strips. Meanwhile the thicker, wider, more ductile gold heater strips did not 
crack or fragment, but suffered from adhesion loss along the entirety of the structure. Combining the observations and measurements from both the D1 and D2 heater process developments, the best way to proceed would be a process amalgam that would return to the sputter deposition process but maintain the gold heater material. Should mechanical fracture still cause the silicon under the bond pads to separate from the device layer, switching to BHF would reduce the structural release shock.

Alternate methods of protecting the D2 heaters during the bridge release process were explored, including PR passivation to protect the heater strips during the bridge release etch, and a BOX dry etch to reduce the HF etch bridge release time regardless of etchant strength. These experiments are summarised in appendix III though none greatly improved the adhesion results for evaporated heaters.

\subsection{Cleaving and Facet Preparation}

Once the heater elements had been deposited, and the bridge etch in the ECR was completed, the next step was to scribe and cleave facets in the waveguide for light to be coupled to. Facet preparation reduced the die size from $7 \mathrm{~mm} \times 12 \mathrm{~mm}$ to $7 \mathrm{~mm} \times 7 \mathrm{~mm}$, suitable for mounting in a chip carrier for prototype packaging.

Facet preparation is usually the final step to be completed in the fabrication lab, and is considered part of the post-process. This is partially due to the fact that a minimum amount of handling is desired once the facets have been exposed to the potential of chipping or abrasion. For this device, however, conducting the bridge release post-process before facet preparation would leave the suspended bridges vulnerable to fracture from the physical contact that occurs on the die surface during the scribing and cleaving process. 
A silicon waveguide that is improperly cleaved will leave conchoidal fractures and other surface anomalies that will scatter light impinging on its facet. To minimize this outcome a common procedure is to flat-lap the SOI handle layer to approximately half its original thickness. A thinner die has an increased likelihood of cleaving easily, leaving an optically smooth waveguide facet. Flat-lapping the die requires bonding the device layer of the SOI to a lapping block. This process could damage structures patterned on the device layer and is fairly laborious.

A procedure was developed that allowed for a highly reliable direct scribe and cleave of the device waveguides. Two straight edges (in the form of a glass slide) were aligned parallel and perpendicular to the (110) crystallographic plane. This ensured that the perpendicular slide would align parallel to another $\{110\}$ plane and form a straight edge the scribe could travel along. A hex driver shaft was positioned directly under the scribe. By applying pressure with finger tips on either side of the scribe, the shaft edge was used as a fulcrum to focus the cleave directly along the scribe line, as shown in figure 5.33 .

This resulted in a controlled, optically smooth cleave, as shown by the examples in figure 5.34. Cross-section image a) shows control over placement of a cleave along the length of an eight micron wide Bragg grating. In the SEM micrograph it is possible to see the entire $2.5 \mu \mathrm{m}$ device layer thickness with the BOX layer underneath it. Images b) and c) in figure 5.34 show typical waveguide facets created by this aligned scribe and cleave procedure. The author presented this technique using the crystallography of silicon to explain the process in a half day course offered by Algonquin College, Carleton University, and NRC [101]. 

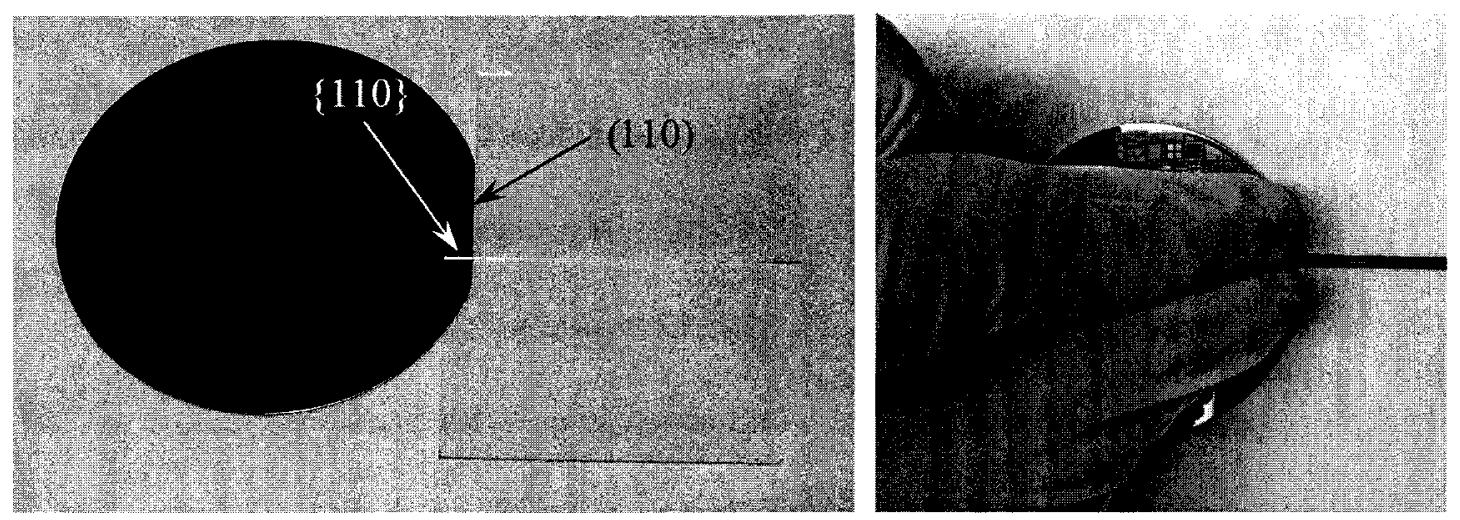

Figure 5.33. Aligning the scribe straight edge to $\{110\}$ planes for scribe and cleaving.

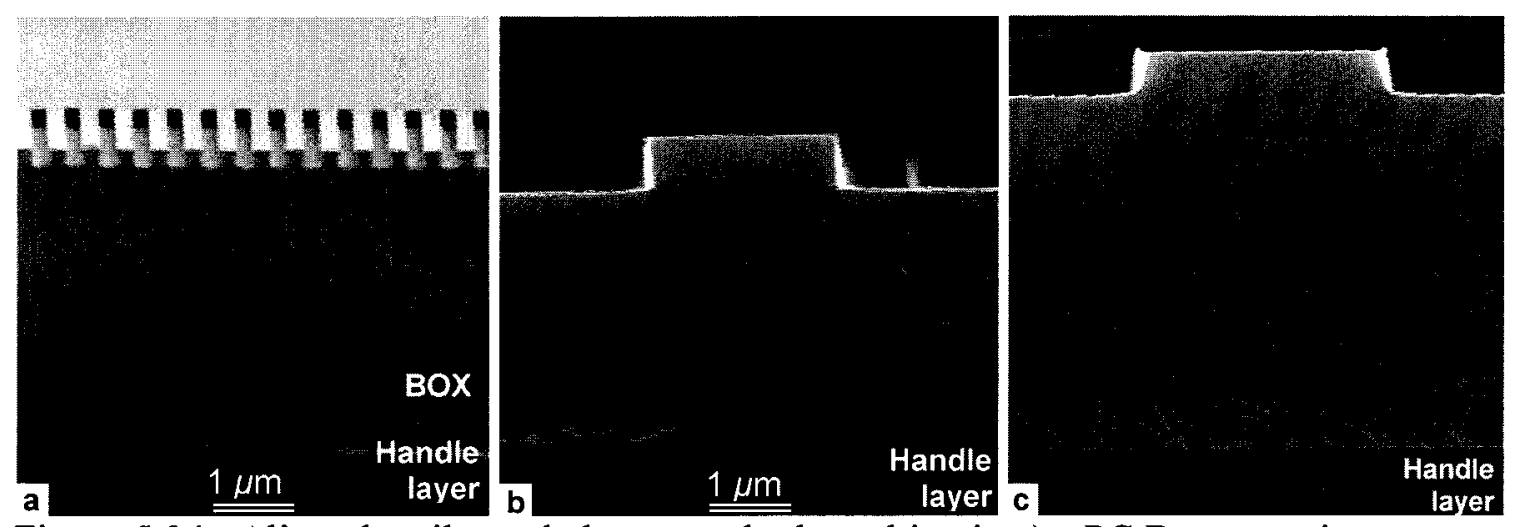

Figure 5.34. Aligned scribe and cleave method resulting in a) a PC Bragg grating crosssection, b) a D1 rib waveguide facet, and c) a D2 rib waveguide facet.

\subsection{Wet Etch Bridge Release Post-process}

With facet preparation complete, the next step in the process flow was the bridge release post-process. Four release techniques were explored in the D1 stream and one technique was carried over and refined further in the D2 stream. The D1 stream used SIMOX (Separation by ion implantation of oxygen) SOI with a $2.5 \mu \mathrm{m}$ device layer of single crystal silicon (SCS) and a $1 \mu \mathrm{m}$ BOX layer. The D2 stream used BESOI (Bonded and Etched-back SOI) wafers featuring a $5.0 \mu \mathrm{m}$ device layer of SCS and a $2.0 \mu \mathrm{m} \mathrm{BOX}$ layer of thermal oxide.

A common source of failure during the release of a surface micromachined structure is due to stiction [102]. When a wet etchant is used in the sacrificial release 
post-process, the liquid must be removed once its task is complete. A meniscus will form between the substrate and structural surfaces as the liquid evaporates, and the resulting capillary force can pull the released structure towards the substrate. If the structure should make contact with the substrate, both of which are highly smooth and clean, van der Waals force will likely bond the two surfaces together, causing the structure to permanently collapse [103].

\subsubsection{Bridge Release Process Development}

The D1 bridge mask contained a series of bridge lengths from $100 \mu \mathrm{m}$ to $4000 \mu \mathrm{m}$. This progression of lengths made a good test bed to track progress in the process development as longer bridges were released over time. Initial experiments were fundamental in developing and practicing handling procedures for the $7 \mathrm{~mm} \times 7 \mathrm{~mm}$ die.

Process steps that were common to all techniques included an initial HF wet etch to release the silicon waveguide bridge from the sacrificial buried oxide. Once a timed etch was complete and the bridge was released from the sacrificial oxide, the die was transferred to a one litre Petri dish of deionised water (DI). To maintain the degree of control necessary for proper handling of the die, a normal DI cascade could not be used, but as large a quantity of DI as possible was desired to dilute and replace the HF. After a five minute bath the die was moved to a Petri dish of isopropyl alcohol (IPA) for three minutes. This step replaced the DI with a solvent. The next Petri dish in the flow contained HMDS. When HMDS is adsorbed onto a surface with Si-OH groups, it produces a monolayer of methyl groups on the surface, which in turn reduces the tendency of the surface to hydrogen bond. This reduces the influence of secondary bonding forces that contribute to stiction [104,105]. 
Die were handled along the edge, parallel to the waveguides, with flair nosed tweezers to avoid any contact with surface structures or waveguide facets. This also allowed for the horizontal placement and removal of the die into each of the baths of the wet etch procedure as shown in figure 5.35. With the exception of removal of the die from the DI step (where the surface was now hydrophobic), this ensured that a dome of liquid would form over the die due to surface tension. This dome would stay in place during transport to the next bath to prevent the fluid under the bridge from evaporating. This was particularly important with the final wet steps that involved volatile solvents, which would evaporate rapidly under a fume hood.

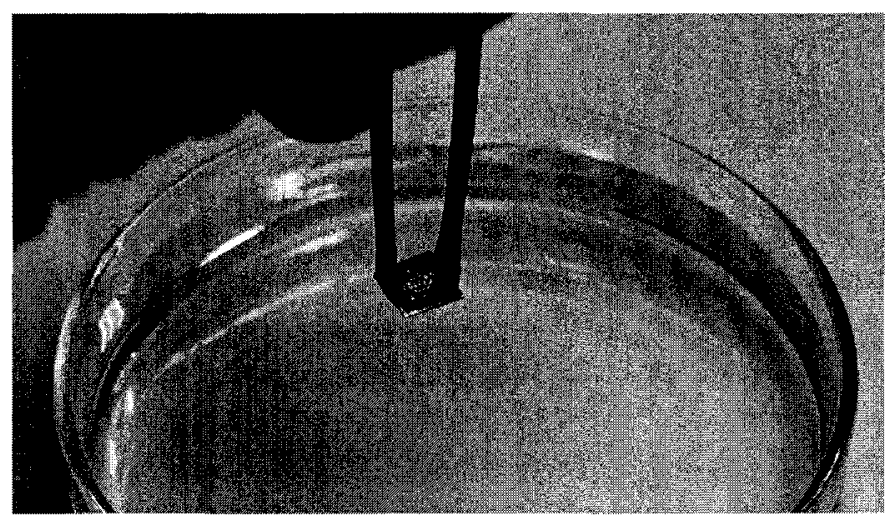

Figure 5.35. Handling technique for die during the bridge release process

The primary method of inspection microscopy for a successful bridge release was phase contrast illumination. A microscope using a polariser and a quarter wave plate in the objective lens would highlight any difference in the distance from the sample surface to the lens with a change in surface profile brightness. This configuration produced better photomicrographs than dark field microscopy could.

As figure 5.36 illustrates, a difference in brightness on the silicon surface indicates a change in surface profile. This was useful to delineate the undercut of the sacrificial oxide from the supported silicon device layer, also known as the etch front. A 
bridge that had collapsed would also show a marked change in brightness along the length of the structure, while a bridge that was successfully released would show a consistent intensity along its length. The samples in Figure 5.36, produced during the early stages of the bridge release development, show the successful release of a $100 \mu \mathrm{m}$ and $200 \mu \mathrm{m}$ bridge and the collapse of a $400 \mu \mathrm{m}$ and $800 \mu \mathrm{m}$ bridge. SEM scans were initially used to confirm what was being detected optically.

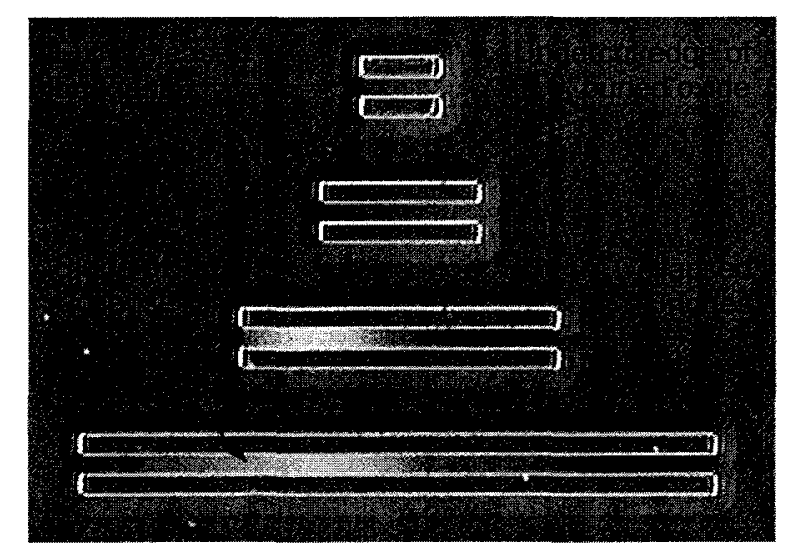

Figure 5.36. Photomicrograph showing the release of a 100 and $200 \mu \mathrm{m}$ bridge and collapse of a 400 and $800 \mu \mathrm{m}$ bridge. The etch front between oxide and silicon is even.

This method of inspection was a rapid and accurate way to determine immediately after sacrificial release if a bridge had collapsed. The inspection technique was particularly useful during the initial post-process development. Samples could be inspected, in situ, during the IPA step by placing the Petri dish containing the solvent on the microscope stage. It was possible to confirm that the full compliment of bridge structures, from $100 \mu \mathrm{m}$ to $4000 \mu \mathrm{m}$, were still free standing, and that the techniques developed for handling and removal of the test die from HF or DI did not cause structural collapse. Another technique for such inspection has been reported using a near-IR camera to detect etch fronts in $\mathrm{SiO}_{2}$ [106], but the technique described here proved just as 
capable and made use of available equipment.

Initial experiments determined the success rate when air drying a released bridge. This process resulted in bridges longer than $100 \mu \mathrm{m}$ collapsing. A $100 \mu \mathrm{m}$ long $2.5 \mu \mathrm{m}$ thick bridge has a length to thickness aspect ratio of 40:1. Techniques were investigated to re-release a collapsed bridge. Mechanical separation from the handle layer of the SOI using a probe tip would only rupture the silicon bridges. Ultrasonic vibration would fracture all unsupported silicon, leaving only the collapsed portion of the bridge behind.

Success has been reported for freeing a stuck structure using such techniques as rapid thermal annealing or ultra-short laser pulses [107,108]. A variation was attempted during the test and characterisation work by setting the laser used for external heating to its maximum power and cycling it at $100 \mathrm{~Hz}$, but this approach had very limited success.

After simple evaporation had been ruled out as a bridge release technique, sublimation was explored next. Sublimation occurs when a material in a solid phase is taken directly to its gaseous state. In doing so, the problem with surface tension and pullin force as the liquid evaporates can be avoided. 2-methyl-2-propanol, an alcohol with a melting point of $25^{\circ} \mathrm{C}$, was used. A small metal tray on a hotplate, set just above $25^{\circ} \mathrm{C}$, was used to hold the alcohol. After the bridge release process was complete the die was transferred to the alcohol and the tray was placed in a refrigerator for solidification. Next the die was transferred to a vacuum chamber. When the chamber was evacuated the solid alcohol sublimed to a gas, bypassing the liquid stage. Experiments yielded successful bridge releases up to $400 \mu \mathrm{m}$ long with an aspect ratio of 160:1.

The next technique to be investigated was super critical drying. In order to pursue this avenue, the Tousimis critical point dryer (CPD) at the McGill University Microfab 
was used. Since the die size was too small to fit in the reaction chamber die holder, the die were mounted on a $100 \mathrm{~mm}$ silicon wafer using a high temperature black wax. This wax was resistant to reflowing under pressure and was also resistant to HF.

Once the wet etch bridge release had been completed, the wafer was transferred to an IPA bath and mounted in the reaction chamber. Liquid $\mathrm{CO}_{2}$ replaced the IPA under high pressure in the sealed chamber. The sample was then heated under high pressure until the $\mathrm{CO}_{2}$ was beyond its critical point between liquid and gas. At this stage of the process there was no difference in the density of $\mathrm{CO}_{2}$ in its liquid and gaseous state. The liquid became a gas without crossing a phase boundary and therefore the surface tension normally present when a liquid becomes a gas was avoided. With no surface tension, structural collapse due to pull-in force was avoided.

Die were removed from the black wax by heating the backing wafer above $75^{\circ} \mathrm{C}$ and then sliding the die slowly off the wafer so the wax was left behind. This process yielded a successful bridge release on D1 die up to $2000 \mu \mathrm{m}$ long with an aspect ratio of $800: 1$.

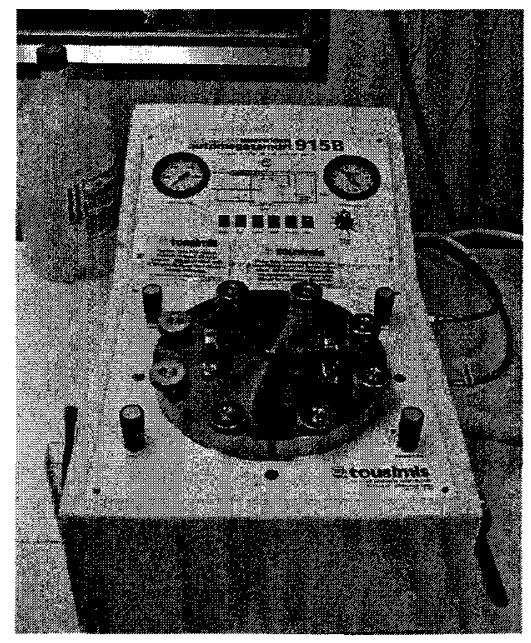

Figure 5.37. Tousimis critical point dryer present at the McGill University Microfab. 


\subsubsection{Oxygen Plasma Dry Release with PR support}

The final bridge release technique developed would replace the sacrificial wet etchant with a supportive PR film [109]. Once in solid form, the dry reactive chemistry of the oxygen plasma would break down the PR while leaving the microstructural material unaffected. The process was first attempted in the D1 stream, but was refined and utilized in the D2 stream.

This release technique required adding two more steps to the wet etch release process already described. After the HMDS dip, the die was placed in an acetone bath. Acetone is an excellent solvent for PR and would assist in the infiltration replacement process. When the die was removed from the acetone, the tip of an eyedropper holding the PR was placed along the side of one of the tweezer arms to allow a controlled flooding and acetone replacement with the PR. After the PR flood, the die was placed onto a 2 inch backing wafer where it was air dried under a fume hood. Finally, a scalpel was used to remove the die from its backing wafer so the plasma ash bridge release could be performed.

To develop the process, a series of experiments were conducted in which the photoresist was baked at various temperatures and spun on at various speeds. Different viscosities of photoresist were also tested, including S1811 and S1805; S1811 being the more viscous of the two. It was found that baking the die at any temperature would cause the acetone, and solvents present in the photoresist itself, to flash off faster than the photoresist could cure to hold the bridges in place. A low spin speed of $1000 \mathrm{rpm}$ was predicted to help the photoresist permeate further under the bridge, but centripetal force would merely draw the liquid photoresist out to the edges, causing the same pull-in effect that evaporating liquid would produce. S1805 was predicted to be the best PR choice 
since its less viscous quality would allow further penetration, but actual experiments showed that the thicker S1811 provided more structural support once cured properly.

Flooding the die with S1811 and air curing it for three days produced the best results. Figure 5.38 shows the cross-section of a incompletely released bridge from the D1 stream with partial backfill infiltration of PR. An additional observation was made from this etch profile of the sacrificial oxide. An anisotropic profile is indicated rather than the isotropic profile one would expect from an oxide wet etch. This phenomena would be more closely examined during the $\mathrm{D} 2$ process development.

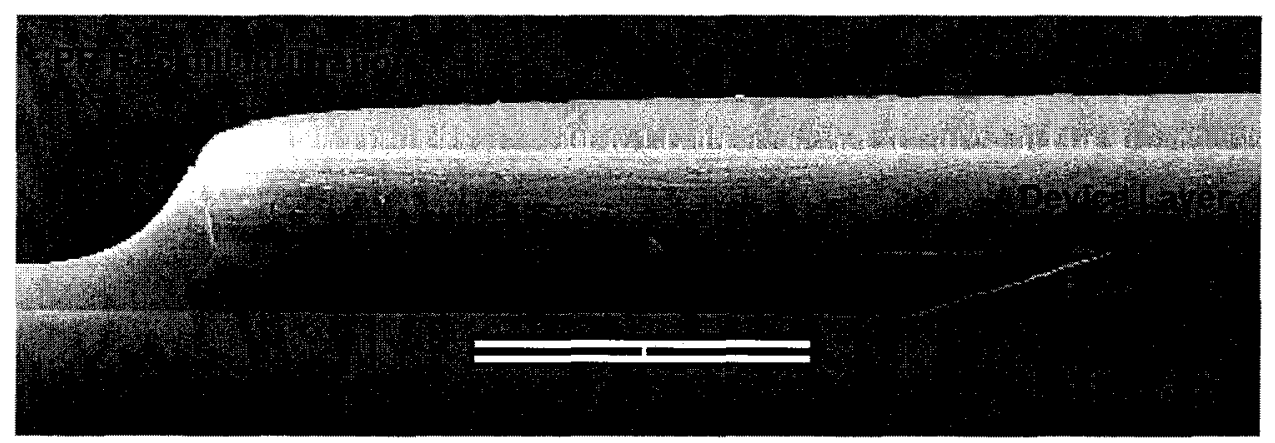

Figure 5.38. Cross-section of D1 device showing PR infiltration from the backfill process for a partially released bridge. An $800 \mathrm{~nm}$ rib waveguide has been etched, leaving a visible device layer thickness of $1.7 \mu \mathrm{m}$.

The Plasma Preen etcher/cleaner was used to ash and remove the PR. The die was sealed within a quartz reaction chamber, which was pumped down to a vacuum of 4.0 Pa. Oxygen gas was then introduced to the chamber at a pressure of $670 \mathrm{~Pa}$ with a flow of $300 \mathrm{sccm}$. Once the oxygen plasma was ignited at $700 \mathrm{~W}$ of power, the solid PR was ashed and removed via the vacuum pump, leaving the bridge structures released and free-standing.

Experiments using this technique yielded successful bridge releases up to $800 \mu \mathrm{m}$ long with an aspect ration of $320: 1$. In the D1 stream, Bragg gratings were only present 
on the $2000 \mu \mathrm{m}$ and $4000 \mu \mathrm{m}$ bridges. Therefore the CPD at McGill was used to process the $\mathrm{D} 1$ die to completion. Multiple runs on this equipment would be required to complete development of the final device. This would add travel time, equipment usage costs, and other considerations to the process development. It was still desirable to pursue a bridge release post-process that could be completed in the Carleton fabrication laboratory.

Experimental results from the D1 process flow were used in the design of the D2 device. The device layer thickness was increased from $2.5 \mu \mathrm{m}$ to $5 \mu \mathrm{m}$. This would give the bridge more stiffness and increase its release length from the current $800 \mu \mathrm{m}$ limit using the sacrificial PR post-process release. The bridges for the D2 device, described in mask design section 4.5 , were set to four lengths: $500 \mu \mathrm{m}, 1000 \mu \mathrm{m}, 2000 \mu \mathrm{m}$, and $4000 \mu \mathrm{m}$.

Two BESOI wafers from Ultrasil Corporation were obtained for the D2 process development. One wafer was used to develop the complete process flow of the D2 device, while the other was used to continue the bridge release post-process development. With a thicker $2.0 \mu \mathrm{m}$ BOX, complete infiltration replacement of the PR was possible, as shown in figure 5.39. The PR in this sample is $21 \mu \mathrm{m}$ thick. 


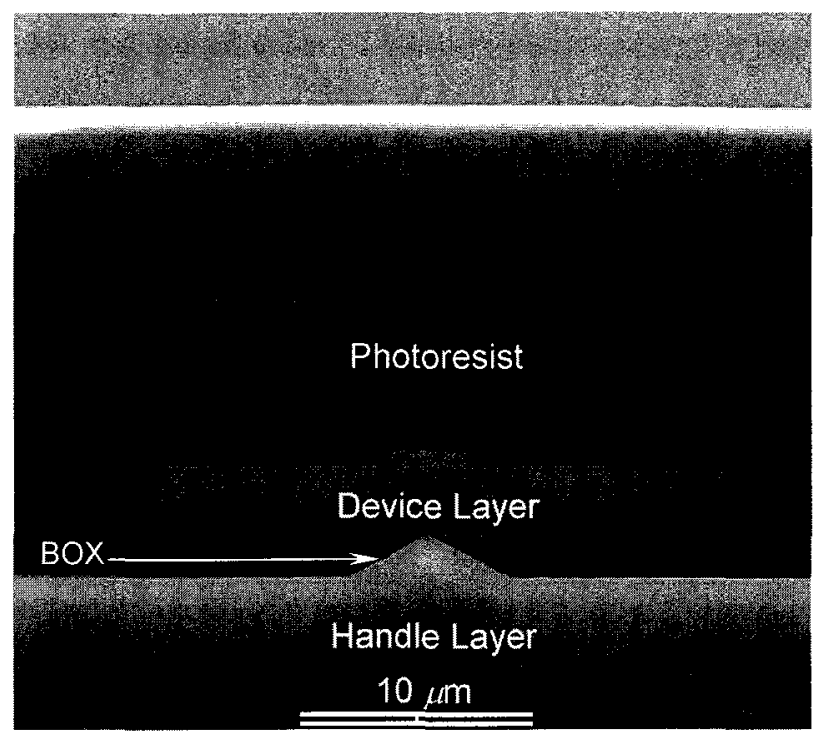

Figure 5.39. Cross-section showing complete PR infiltration replacement for a partially released waveguide bridge. PR thickness is $21 \mu \mathrm{m}$.

Once solidified, the PR would hold a released bridge so securely that even the mechanical shock of cleaving a sample for cross-section inspection would not rupture the silicon structure. The bridge would be held in place, each side forming a cantilever. This was observed for the full range of bridge lengths. Figure 5.40 shows the cleave and cross-section of a $500 \mu \mathrm{m}$ long released bridge after the PR support was ashed away.

The bridge release post-process conducted on the two BESOI wafers revealed that the sacrificial $\mathrm{BOX}$ in the bridge test wafer etched with an " $\mathrm{A}$ " profile while the sacrificial BOX in the device wafer etched with a " $\mathrm{V}$ " profile as shown in figure 5.41. In order to release a microstructure from the "A" profile SOI, a complete removal of the sacrificial layer was unnecessary. For complete release from the "V" profile BOX, the entire sacrificial layer would have to be removed during the etch in order that no oxide remain under the microstructure to influence its mechanical behaviour. 

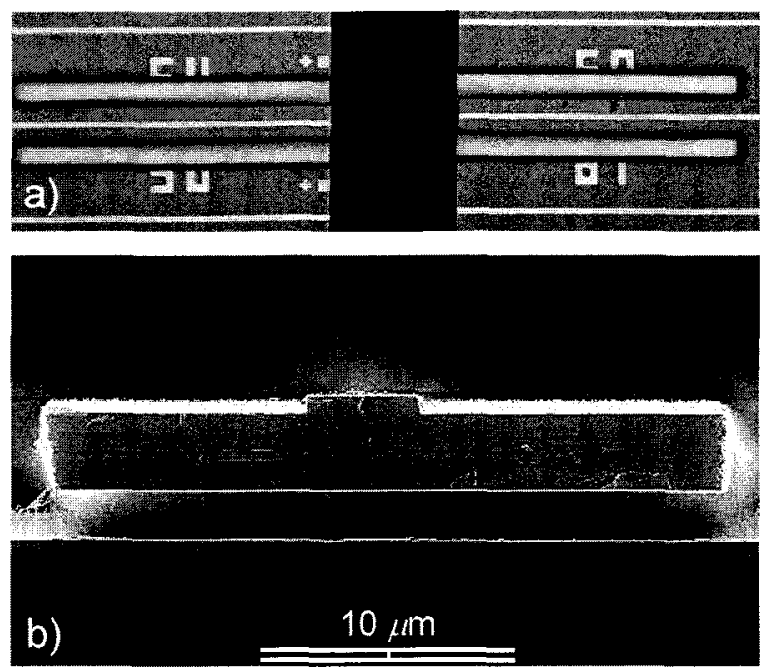

Figure 5.40. a) top down photomicrograph, and b) SEM cross-section of the same 500 $\mu \mathrm{m}$ long bridge after the ash release from its PR support. Structures were held in place without fracture during the cross-section cleave.
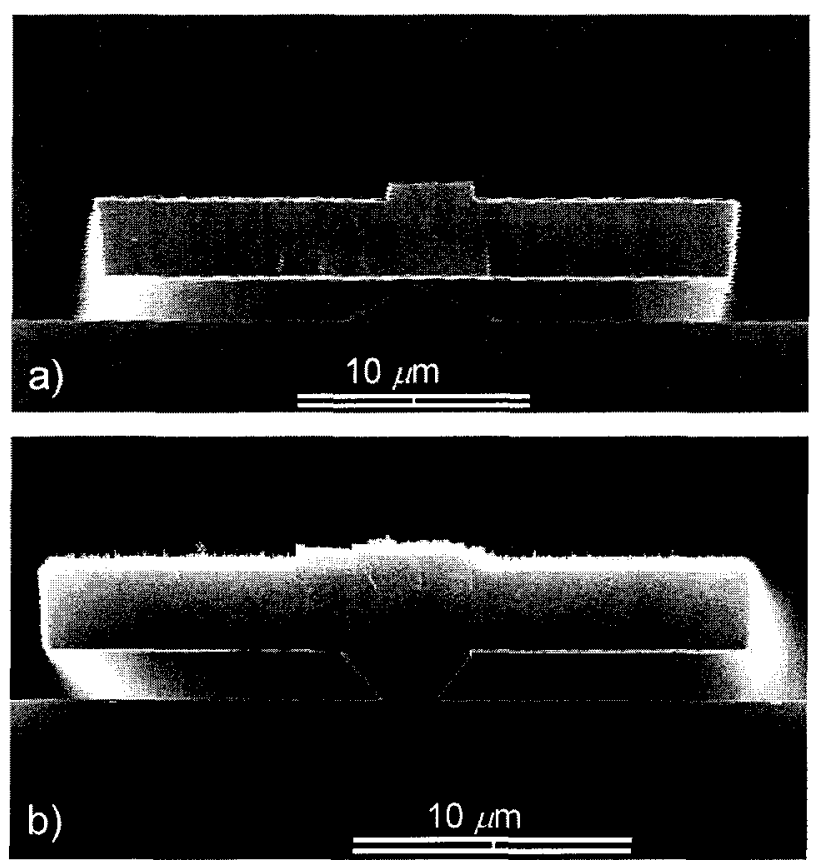

Figure 5.41. Cross-section from both BESOI wafers. a) shows an "A" release profile from the bridge test wafer and $b$ ) shows a " $V$ " release profile from the device wafer.

The difference between the "A" and "V" profile is explained by the work of S. Ponoth et al. [110]. A film with uniform composition throughout will yield an etch contour that is described roughly by an arc whose radius of curvature lies at the edge of 
the structural layer. The lateral etch rate, $E_{l}$, is the same as the vertical etch rate, $E_{v}$. Figure 5.42 depicts the progress of an isotropic etch profile over four intervals where there has been no agitation of the etchant. The material stack illustrated is typical of a surface micromachining process in which an oxide sacrificial layer is deposited, followed by a structural layer. As the etch proceeds, the arc of the radius of curvature of the sacrificial layer boundary will be close to perpendicular to the structural and substrate layers. If the lateral etch rate is greater than the vertical etch rate, $E_{\mathrm{l}}>E_{\mathrm{v}}$, the etch profile will no longer be isotropic.

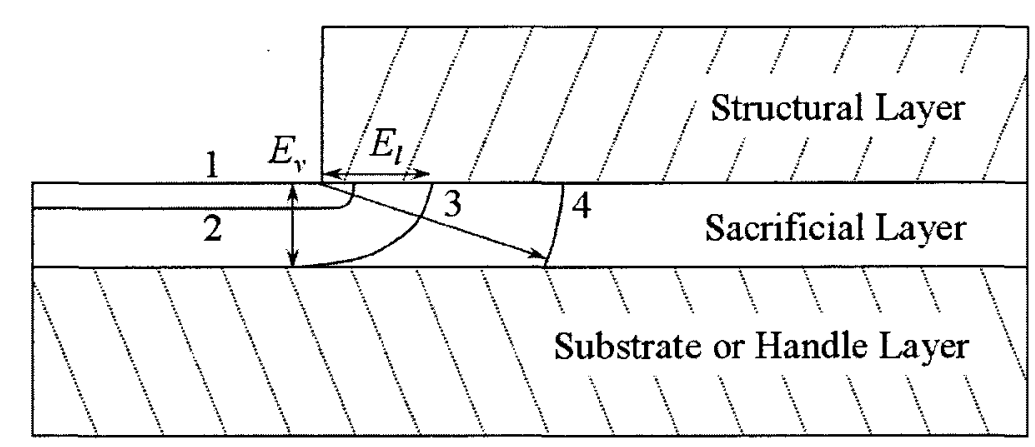

Figure 5.42. Isotropic etch profile over four intervals of a homogeneous sacrificial layer.

Figure 5.43 depicts the progress of an "A" etch profile over four intervals that results from two separate etch rates, $E_{1}$ and $E_{2}$, within a sacrificial layer where $E_{1}>E_{2}$. When this is accomplished through the formation of two films, the slower etching film is referred to as the working film and the faster etching film is the sacrificial film. The sacrificial layer has, in effect, its own sacrificial film. This can be accomplished through a variety of techniques such as changing the homogeneity of a single sacrificial layer through doping [111], or ion implantation [112]; or by physically forming two films with different etch rates [113]. 


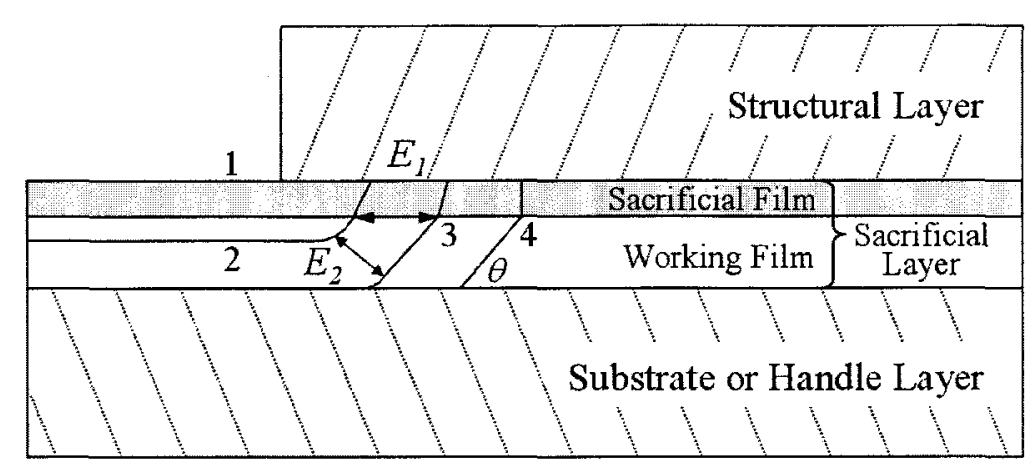

Figure 5.43. An "A" etch profile as it develops over four etch intervals of an inhomogeneous or bi-film sacrificial layer.

To create a "V" etch profile, the bottom layer of the BOX would have to etch at a faster rate than the top interface. To determine the bi-film composition of the "V" profile SOI wafer, a sacrificial etch was conducted on a die from the device wafer and terminated before the steady state etch profile had been achieved. The cross-section shown in figure 5.44 reveals that, indeed, the thick thermal oxide had been grown on the device layer wafer instead of the handle (or substrate) layer wafer. The second film residing on the handle/BOX interface likely resulted from fusing the wafers during the BESOI manufacturing process.

Using Figure 5.43 as a guide, the sacrificial and working film order is reversed. Since the working film was exposed to the etchant first, instead of the sacrificial film, a radius of curvature profile similar to that of an isotropic etch can still be seen in the working film of this truncated etch. The "V" profile would develop once the faster etching sacrificial film was exposed and overtook the working film, and a steady state was achieved.

Further inspection of samples from the "A" release bridge test SOI wafer showed that unlike the perfect "A" profile suggested by figure 5.39 or figure 5.41 , the real profile was a meandering peak as shown in figure 5.45. Once the etch rate for the " $\mathrm{A}$ " profile 
sacrificial layer had been experimentally determined, the etch could be terminated after the structural release from the sacrificial film but before complete removal of the working film. This would allow oxide bumps to remain on the handle layer to serve as built-in stand-offs to help prevent structural collapse. When a successfully released $4000 \mu \mathrm{m}$ beam from the "A" profile SOI was thermally actuated so that it buckled out of plane, as shown in figure 5.46 , a line of oxide bumps remaining on the handle layer were revealed.

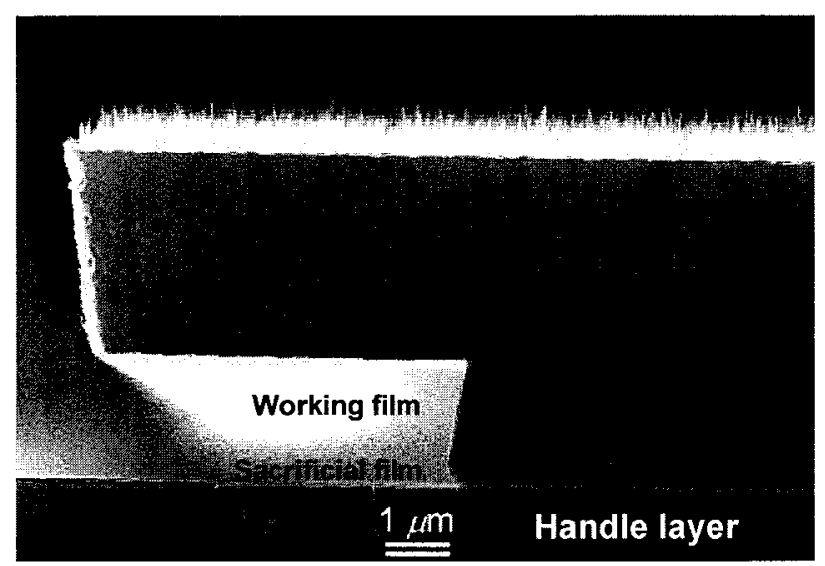

Figure 5.44. Truncated sacrificial etch of the "V" sidewall profile BESOI showing composition of working film and sacrificial film. Once the faster etching sacrificial film overtakes the working film, a "V" profile would develop.
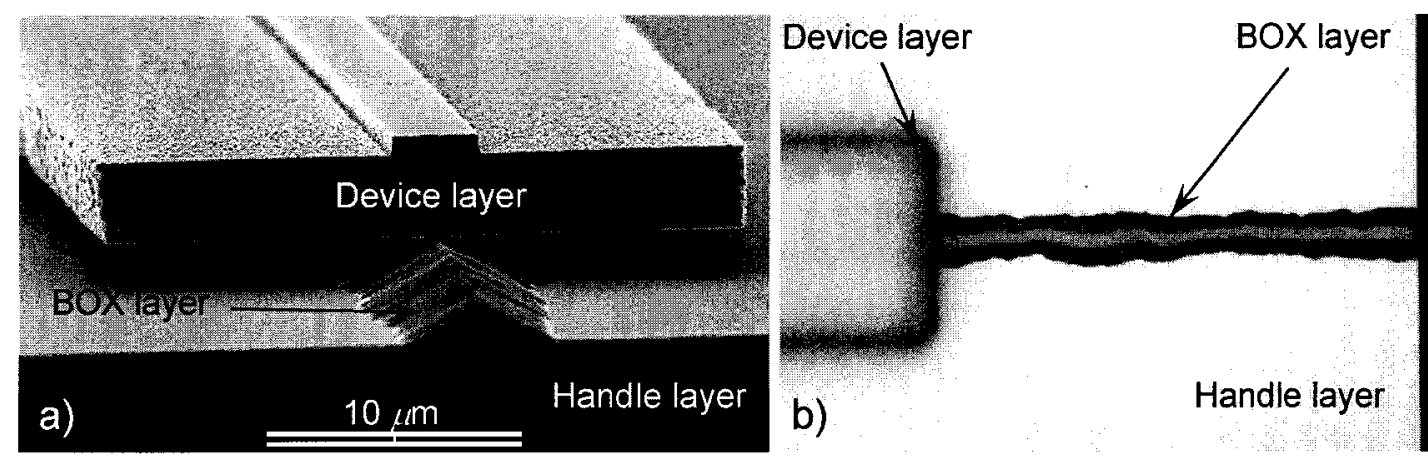

Figure 5.45. True meandering shape of the "A" sacrificial layer SOI shown in a) SEM cross-section and b) top-down photomicrograph of same sample.

The isotropic etch rate for thermal oxide is $2300 \mathrm{~nm} / \mathrm{min}$ for HF [100]. The "A" profile SOI had a faster $E_{2}$ etch rate of $2800 \mathrm{~nm} / \mathrm{min}$. Since the device layer interface of silicon to oxide has a faster lateral etch rate than the working film alone, the oxide is open 
to etching from both the top and side directions. The resultant etch rate yields a faster removal of the sacrificial thermal oxide than is reported by unidirectional or isotropic removal. A 6 minute etch was required to release a bridge on "A" profile SOI that still left behind oxide bumps on the handle layer of silicon. The "V" etch profile changed throughout the bridge release so a constant etch rate could not be measured. Experimentation determined that a 17.5 minute etch was needed to release the bridge and completely remove the oxide from under the structure when using " $\mathrm{V}$ " profile SOI.

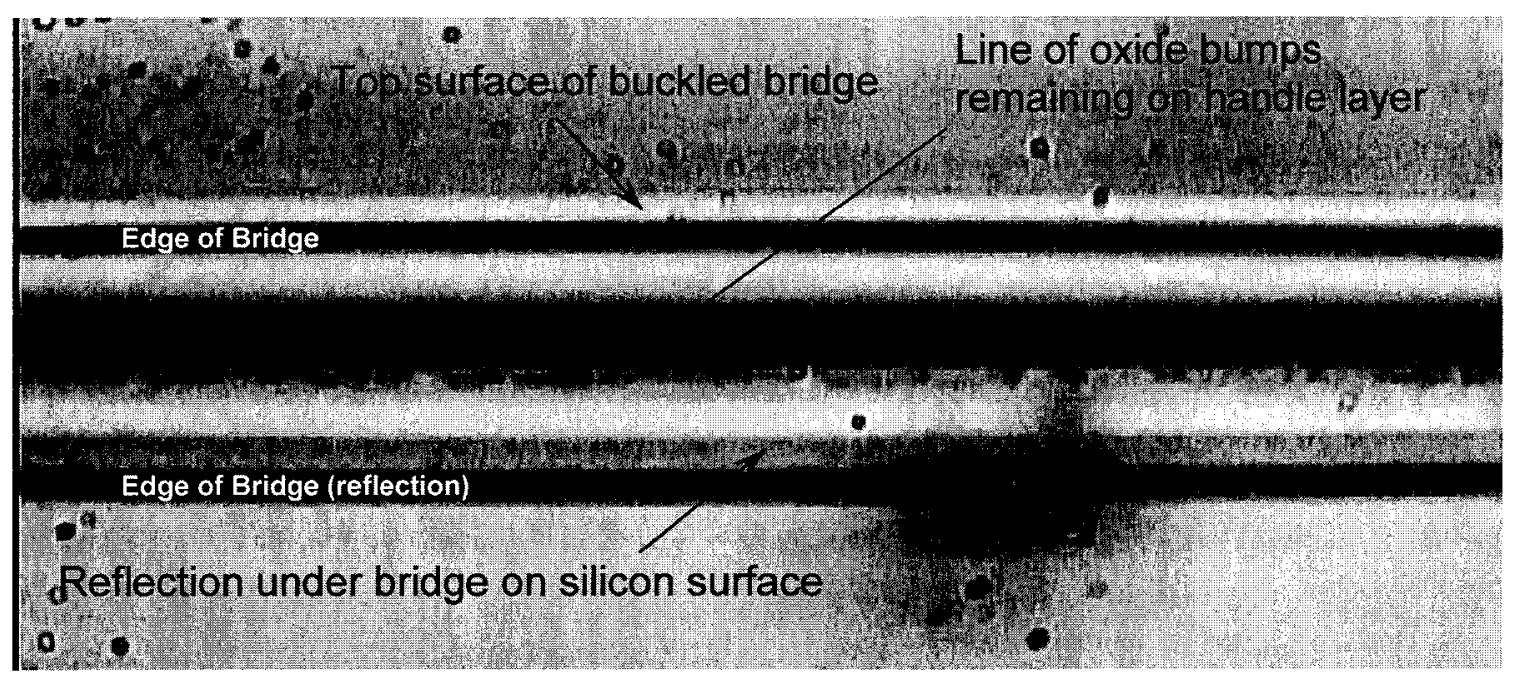

Figure 5.46. Photomicrograph of D2-41 ("A" profile SOI ) showing buckled $4000 \mu \mathrm{m}$ bridge with oxide bumps remaining on the handle layer ( $250 \mu \mathrm{m}$ field of view).

Experiments established that the "A" profile SOI sacrificial etch resulted in an $83 \%$ success rate for the release of $4000 \mu \mathrm{m}$ bridges to their free-standing condition; whereas no $4000 \mu \mathrm{m}$ bridges were successfully released using the "V" profile SOI. Both profile types had nearly a $100 \%$ success rate with $2000 \mu \mathrm{m}$ bridges and shorter. All process and handling techniques remained the same for the "A" and "V" profile SOI and the device layer and BOX dimensions were identical. Therefore the oxide "stand-off" strip more than likely played a role in the successful release of the $4000 \mu \mathrm{m}$ bridge 
structure. With a $5 \mu \mathrm{m}$ device layer thickness, the aspect ratio was 800:1.

A variety of successfully released $4000 \mu \mathrm{m}$ bridges are shown in figure 5.47 . In SEM a) the separation gap of the bridge to the handle layer below is the same as the surrounding silicon, indicating there is no residual stress present in the released microstructure. SEMs b) and c) indicate a release where residual stress in the device layer has caused the bridge to bow slightly out of plane. The $2000 \mu \mathrm{m}$ and shorter bridges on the same die showed no deflection, providing further indication of the small residual stress in the device layer of the BESOI wafers.
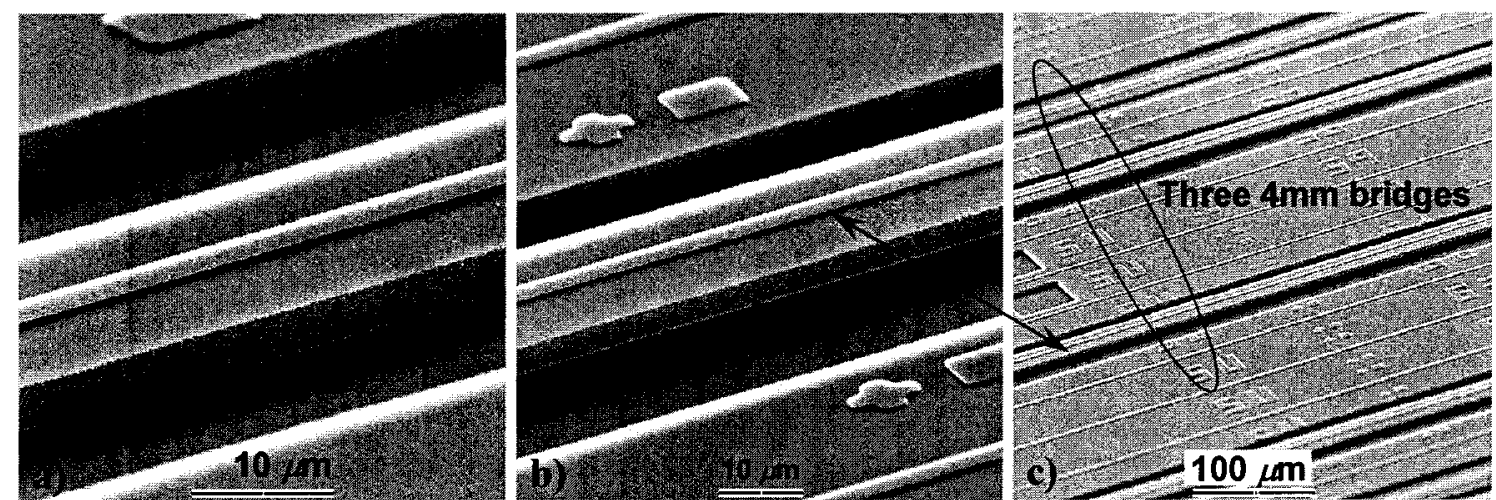

Figure 5.47. Various $4000 \mu \mathrm{m}$ released bridges: a) shows a neutral release. b) and c) show release of a $4000 \mu \mathrm{m}$ bridge with some residual stress in the device layer causing slight out-of-plane bowing.

\subsubsection{Structural Release Shock and Etch Profile}

When the D1 bridges had been processed in the CPD system, inspection revealed that the release process had caused damage to the heaters. This was detailed in section 5.3.3. While the sputtered $\mathrm{NiCr}$ bond pads adhered well to the silicon surface, in many cases the silicon itself ruptured around the perimeter of the bond pad and broke away. This behaviour has been described by J. Kiihamäki in his thesis on the fabrication of SOI micromechanical devices [114]. The bond strength between the oxide and device silicon layer affects both the etch rate of the oxide and the mechanical separation of the silicon 
from the oxide during the etch.

As the bridge release process development began on the D2 SOI, the rupture effect on the device layer silicon was present in the " $\mathrm{A}$ " profile SOI as shown in figure 5.48 but not the "V" profile SOI. The etch front defined by the limit of the oxide etch under the device layer silicon was also irregular in the "A" profile SOI. This allowed the HF etchant uneven lateral access to etch out pockets of oxide that were not along the normal etch front, as shown in figure 5.48 and figure 5.49. The uneven etch front was likely a significant contributor to silicon fracture in the D2 release process for "A" profile SOI. This is consistent with Kiihamäki's work. The "A" profile SOI had a weak bond strength between the thermal oxide and device silicon layer it was fused to (thus the "A" etch profile) which allowed the uneven etch front.
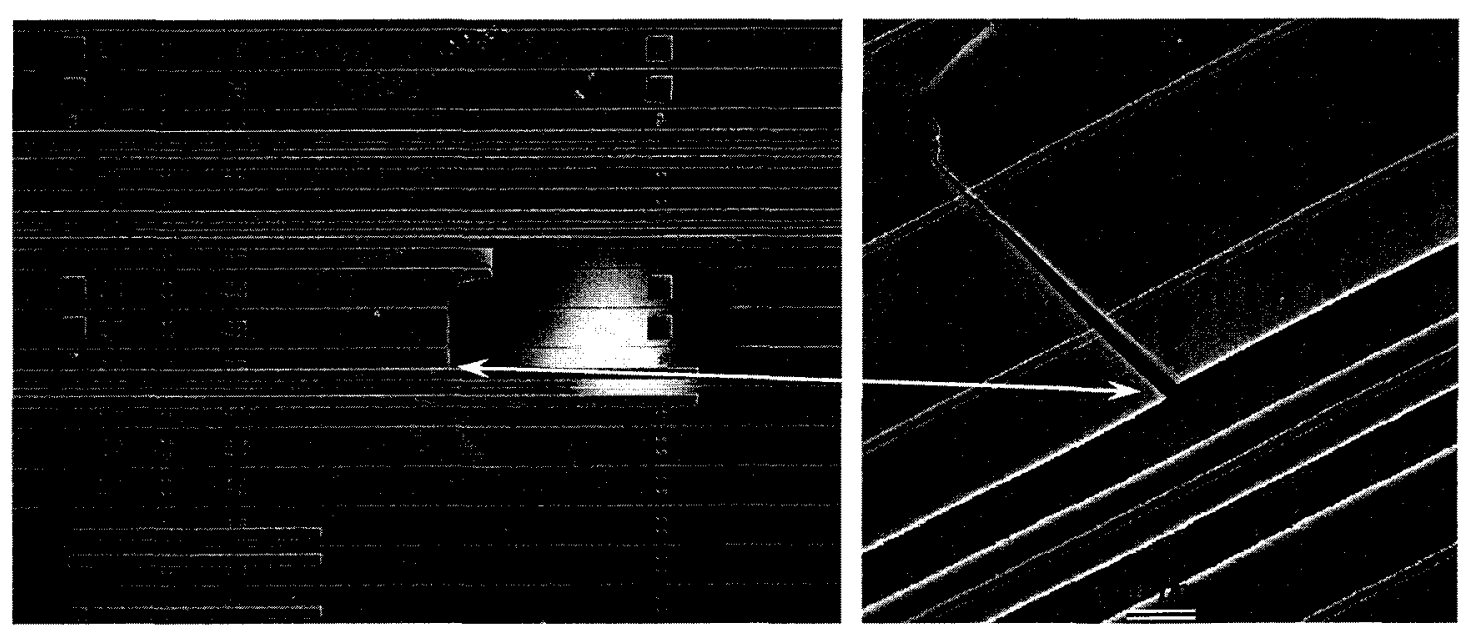

Figure 5.48. Example of mechanical rupture of device layer silicon along an uneven etch front during the sacrificial etch of "A" profile SOI.

By switching from concentrated $49 \% \mathrm{HF}$ to a BHF etchant solution from Transene company, called "Buffer HF Improved", the uneven etch front was eliminated in the "A" profile SOI, but the etch release time was greatly increased from 6 minutes to 95 minutes. Over this length of etch even $500 \mu \mathrm{m}$ and $1000 \mu \mathrm{m}$ gold heaters did not 
remain adhered to the silicon substrate. Minimizing etch time was an important factor to consider to increase the survival rate of the heaters.

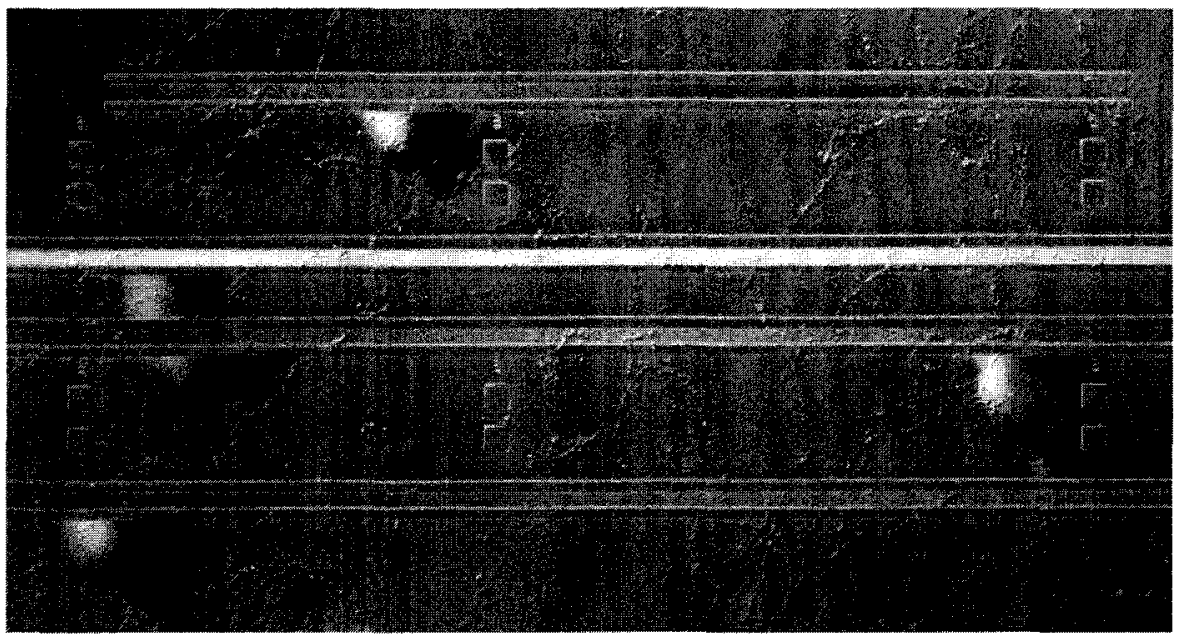

Figure 5.49. Uneven etch fronts left by conc. $49 \% \mathrm{HF}$ on $\mathrm{D} 2$ bridge release process development "A" profile SOI.

\subsection{Packaging and Wirebonding}

Normally a test die undergoing optical characterisation will rest on a test stage or test fixture as optical fibres are aligned to the waveguide facets of the device. Further physical contact with the die after this alignment is undesirable since even submicron perturbation will cause optical misalignment between the fibres and their respective waveguides. When contact probes are used to interface with other components on the die there will always be some instability caused by the physical contact the probe tips make on the surface of the die.

Completed D1 and D2 device die were mounted into an integrated circuit chip carrier so that the integrated heaters could be wirebonded to interface pins. These pins allowed electrical signals to be routed to the integrated heaters without contact probes. Electrical contact to the heaters was physically moved from the die surface to standard 
micro test clips that connect to the pins of the chip carrier, while the package itself was held securely in place with a standard carrier socket or affixed directly to the test stage. This simplified the test setup and stabilised the experimental process, which lead to a greater degree of confidence and repeatability in the characterisation process.

The prototype packaging post-process began by mounting a bare die, of equal size to the device die, into the package well. The bare die acted as a shim to raise the waveguide facets of the device die above the carrier lip, providing access for the optical fibres. To fix both the shim and device die to the chip carrier for the wirebonding process, the fixative would have to withstand the $150{ }^{\circ} \mathrm{C}$ to $200{ }^{\circ} \mathrm{C}$ temperature of the wire bonder workholder. Most adhesives contain additives with a flash temperature below the workholder operating temperature. This ruled out cyanoacrylate based adhesives such as "Super Glue", which have a flash point of $80^{\circ} \mathrm{C}$. High temperature epoxies were a possibility, but as these adhesives cure they could unevenly stress the die itself. Permatex "Permanent Strength Threadlocker", with an operating temperature of $150{ }^{\circ} \mathrm{C}$, was chosen as the adhesive. A die affixed with this adhesive could also be removed from the chip carrier without damage by applying a steady temperature above $200{ }^{\circ} \mathrm{C}$. This removal process was useful if the die required further post-processing or cross-sectional analysis.

Due to the extreme flatness of both the shim and device die, care had to be taken in the amount of fixative dispensed to join the two. Any liquid that squeezed out from the edges of the shim/die interface would wick down the length of the waveguide, potentially changing its optical properties. The minute quantity of adhesive required was applied using a micropipette and cured over 12 hours on a hotplate set to $100^{\circ} \mathrm{C}$. Once 
the die had been mounted into the chip carrier, the West-Bond 7400A ultrasonic wire bonder, shown in figure 5.50, wire bonded the integrated heaters of the device to the chip carrier bond pads.

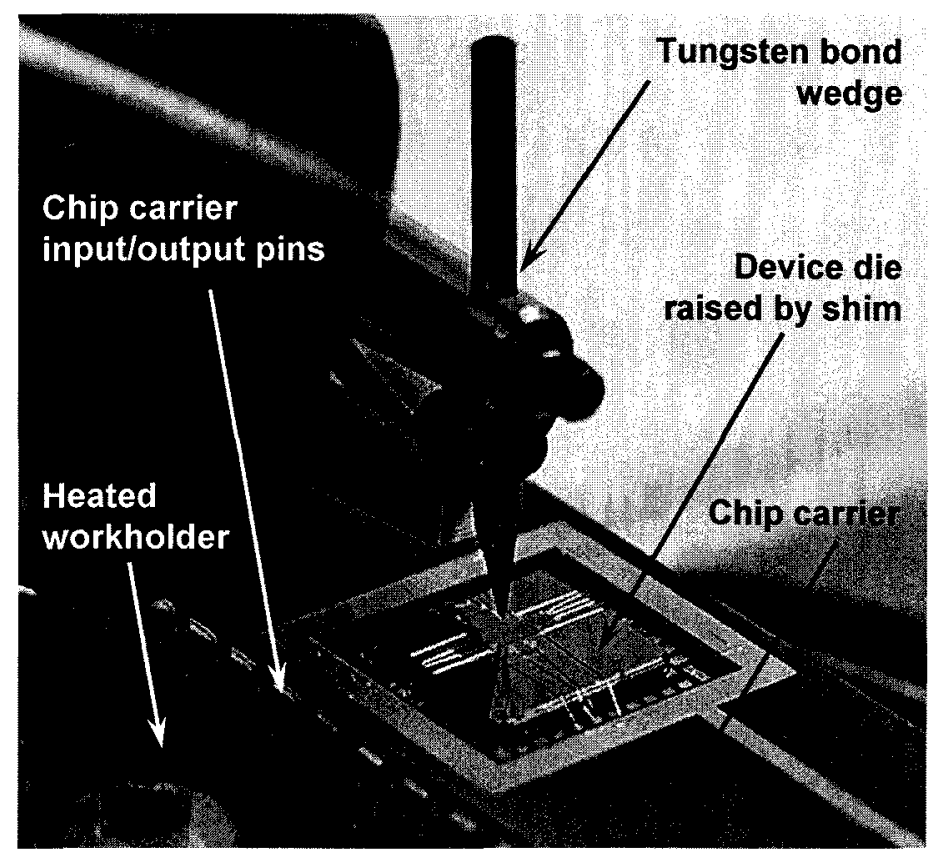

Figure 5.50. West-Bond wire bonder with chip carrier in place on heated workholder for D2-22.

The machine bonded $40 \mu \mathrm{m}$ diameter gold wire from the heater bond pads to the chip carrier pads using a combination of ultrasonic vibration and heat. Heating the metal of the bond pad and bond wire softens the metal, making it easier to deform. When the wire is brought into contact with the bond pad, the wedge vibrates the two metals together in a welding process. A variable combination of bond temperature and vibration power are used depending on the material of the bond pad and bond wire. In the case of aluminum, a greater vibration power is generally needed to ensure penetration through the protective aluminum oxide film that will form on the aluminum surface. When gold is used, a comparatively lower temperature and power compensate for the metal's high ductility and resistance to oxidation. 
If the balance of power and temperature are too low the wire will pull free or contact resistance could become a factor. If the balance of power and temperature are too high damage could occur to the die adhesive, the bond pad, or the wire could be deformed enough to break at the bond point. The result of proper and excessive wire bond settings are shown in figure 5.51 for a gold wire and bond pad. D2-HT1 was prepared, as shown in Figure 5.52, so that electrical characterisation could take place to validate the prototype packaging post-process.

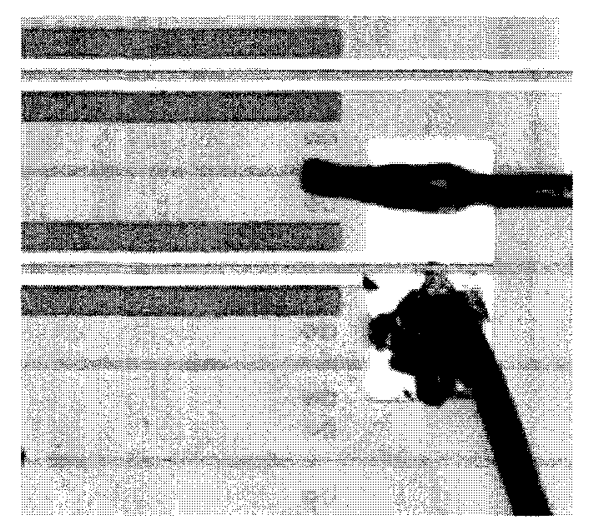

Figure 5.51. Example of proper and excessive wire bond power on D2-22.

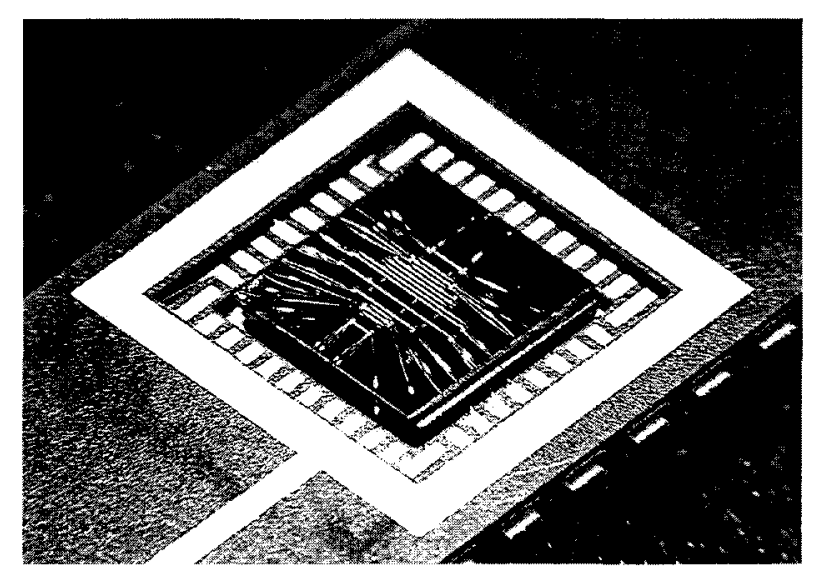

Figure 5.52. D2-HT1 with heaters to test packaging, bonding, and electrical characteristics. 


\subsection{Device Die Completed for Test and Characterisation}

Table 7 has been prepared as a summary of the die processed to completion that were used in the test and characterisation processes described in chapter 6 . In order to complete the summary, the device and BOX layer thicknesses of the SOI platforms are also required. PC and D1 device streams used SOI with a $2.5 \mu \mathrm{m}$ device layer and $1.0 \mu \mathrm{m}$ BOX thickness. The D2 device stream used SOI with a $5.0 \mu \mathrm{m}$ device layer and $2.0 \mu \mathrm{m}$ BOX thickness.

There were a substantial number of process steps required to bring a device from bare wafer to functional device. Over the course of fabrication research, die were spoiled or sacrificed for process flow development; or had structural geometry that varied too much from the device standard being characterised. These die are not shown in this table.

\begin{tabular}{|l|c|c|c|c|c|c|l|}
\hline $\begin{array}{c}\text { Die } \\
\text { Label }\end{array}$ & $\begin{array}{c}\text { Rib } \\
\text { height }\end{array}$ & $\begin{array}{c}\text { Rib } \\
\text { width }\end{array}$ & $\begin{array}{c}\text { Grating } \\
\text { depth }\end{array}$ & $\begin{array}{c}\text { Bridge } \\
\text { width }\end{array}$ & $\begin{array}{c}\text { Heater } \\
\text { width }\end{array}$ & $\begin{array}{c}\text { Heater } \\
\text { thickness }\end{array}$ & Characterisation and testing performed \\
\hline PC-1 & $660 \mathrm{~nm}$ & $2.8 \mu \mathrm{m}$ & $210 \mathrm{~nm}$ & & & & Optical \\
\hline D1-3 & & & & & $4.3 \mu \mathrm{m}$ & $3000 \AA$ & Electrical and packaging \\
\hline D1-6 & $530 \mathrm{~nm}$ & $4.1 \mu \mathrm{m}$ & $470 \mathrm{~nm}$ & & & & Optical, thermomechano-optical \\
\hline D2-HT1 & & & & & $8.0 \mu \mathrm{m}$ & $10000 \AA$ & Electrical and packaging \\
\hline D2-13 & & & & $30.9 \mu \mathrm{m}$ & & & Thermo-mechanical \\
\hline D2-16 & $890 \mathrm{~nm}$ & $5.0 \mu \mathrm{m}$ & $1.0 \mu \mathrm{m}$ & $30.7 \mu \mathrm{m}$ & & & Thermo-mechanical, thermomechano-optical \\
\hline D2-20 & $930 \mathrm{~nm}$ & $5.2 \mu \mathrm{m}$ & $860 \mathrm{~nm}$ & $32.3 \mu \mathrm{m}$ & & & Thermomechano-optical \\
\hline D2-21 & $810 \mathrm{~nm}$ & $4.9 \mu \mathrm{m}$ & $860 \mathrm{~nm}$ & $30.9 \mu \mathrm{m}$ & & & Thermo-mechanical \\
\hline $\mathbf{D 2} 2-22$ & $850 \mathrm{~nm}$ & $4.7 \mu \mathrm{m}$ & $860 \mathrm{~nm}$ & $31.0 \mu \mathrm{m}$ & $7.9 \mu \mathrm{m}$ & $9500 \AA$ & Electro-thermal and thermo-optical \\
\hline D2-38 & $1.4 \mu \mathrm{m}$ & $5.0 \mu \mathrm{m}$ & $1.7 \mu \mathrm{m}$ & $31.8 \mu \mathrm{m}$ & & & Thermo-mechanical \\
\hline $\mathbf{D 2} 2-41$ & & & & $31.2 \mu \mathrm{m}$ & & & Thermo-mechanical \\
\hline
\end{tabular}

Table 7. Summary of device die used in test and characterisation experiments. Unfilled boxes indicate that the device was not processed with the material or structure shown in that column. 


\section{Test and Characterisation}

The electrical, thermal, mechanical and optical behaviour of the device in this thesis was theoretically laid out in chapter 3 . In chapter 4 this theory was used analytically to design the material and structural parameters of the device. Chapter 5 described the fabrication processes that was developed from these parameters to build the devices. This chapter will describe how the device was characterised in order to show that the theoretical analysis is in agreement with its performance.

Initial optical characterisation on the PC device stream was performed by Edith Post at IMS-NRC. A test platform was subsequently established at Carleton University to conduct optical characterisation in conjunction with electro-thermal and thermomechanical testing.

\subsection{Electro-thermal Characterisation}

It was important to validate theoretical resistance for the heaters against the experimental measurements so the heater behaviour could be characterised with confidence. Experimental measurements on the resistance of the heaters packaged in D2-HT1 were compared to the analytic results calculated from theory. Resistivity of the gold used for the D2-HT1 heaters was measured to be $2.69 \times 10^{-8} \Omega \mathrm{m}$. The heater thickness was $1.0 \mu \mathrm{m}$ and the width was $8.0 \mu \mathrm{m}$. Using the resistivity and heater dimensions, the resistance values were calculated using equation 3.13. A Keithley 195A digital multimeter was used to make direct resistance measurements. These results were compared to the calculated heater resistance, as shown in table 8. The percent difference 
between experimental measurement and analytic calculation ranged from $10 \%$ to $19 \%$. The purpose of this comparison was strictly a sanity test for confidence in the devices fabricated. With confidence established for the behaviour of the packaged heaters, the electro-thermal characterisation could be conducted.

\begin{tabular}{|c|c|c|c|c|}
\hline Heater length & $4.0 \mathrm{~mm}$ & $2.0 \mathrm{~mm}$ & $1.0 \mathrm{~mm}$ & $0.5 \mathrm{~mm}$ \\
\hline $\begin{array}{c}\text { Theoretical } \\
\text { Resistance }\end{array}$ & $13.5 \Omega$ & $6.7 \Omega$ & $3.4 \Omega$ & $1.7 \Omega$ \\
\hline $\begin{array}{c}\text { Experimental } \\
\text { Resistance }\end{array}$ & $11.3 \Omega$ & $7.8 \Omega$ & $3.1 \Omega$ & $2.1 \Omega$ \\
\hline Percent Difference & $19 \%$ & $14 \%$ & $10 \%$ & $19 \%$ \\
\hline
\end{tabular}

Table 8. Comparison of theoretical heater resistance to experimental measurement.

The gold heaters had a low resistance. This meant the ohmic contact of the bond pads would have to be characterised to ensure their contribution to the resistance of the heaters was negligible. Some heaters were double wire bonded to the package connectors, as shown in figure 6.1, so that the resistance from the input-pin to output-pin could be compared to the same heaters with a single wire bond.

Double bonding the heaters reduced the total resistance by $1.8 \%$ for the longest heaters, and $4.2 \%$ for the shortest heaters, indicating that the majority of the resistance occurred on the heaters strips alone. The results from the shortest and longest heaters are summarized in table 9. This experiment was not necessary for the NiCr heaters since its resistance, at $1750 \Omega$ for a $2.0 \mathrm{~mm}$ heater, was much greater.

\begin{tabular}{|c|c|c|}
\hline $\begin{array}{c}\text { Ohmic Contact } \\
\text { Comparison }\end{array}$ & $0.5 \mathrm{~mm}$ heater resistance $(\Omega)$ & $4.0 \mathrm{~mm}$ heater resistance $(\Omega)$ \\
\hline Single wirebond & 2.13 & 11.3 \\
\hline Double wirebond & 2.04 & 11.1 \\
\hline Percentage Difference & $4.2 \%$ & $1.8 \%$ \\
\hline
\end{tabular}

Table 9. Comparison of resistance due to ohmic contact. 


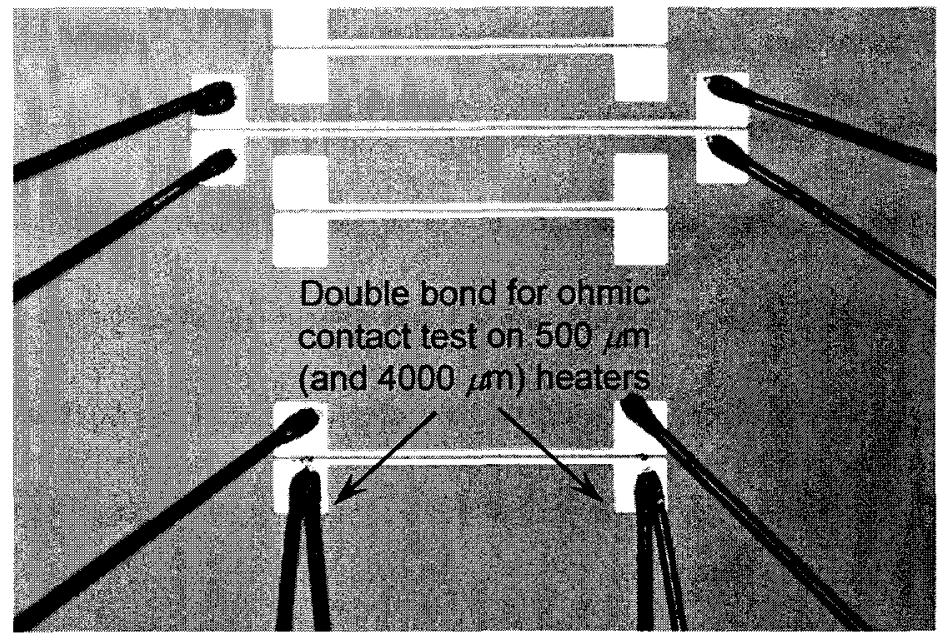

Figure 6.1. Test for ohmic contact of wirebond to heater strips on D2-HT1.

\subsubsection{D1 Device}

Electro-thermal characterisation was carried out using a Xantrex XT20-3 regulated DC power supply to provide DC current to the heaters and a Keithley 195A digital multimeter to measure the voltage across the heater. The experimental schematic is shown in figure 6.2 while the D1 device as it was electro-thermally tested is shown in Figure 6.3.

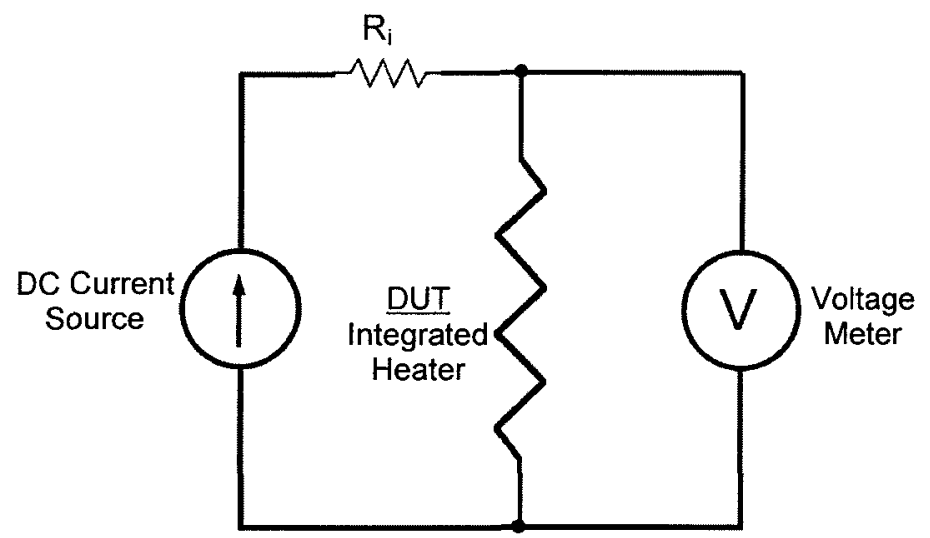

Figure 6.2. Schematic for electrical characterisation of integrated heaters.

While electro-thermal characterisation could be more easily performed on loose die with an electrical probe station, the eventual goal was to couple the electro-thermal characterisation to the mechanical and optical performance. Using the same test bench 
for all stages of characterisation would develop a consistent experimental procedure and increase confidence in measurements.

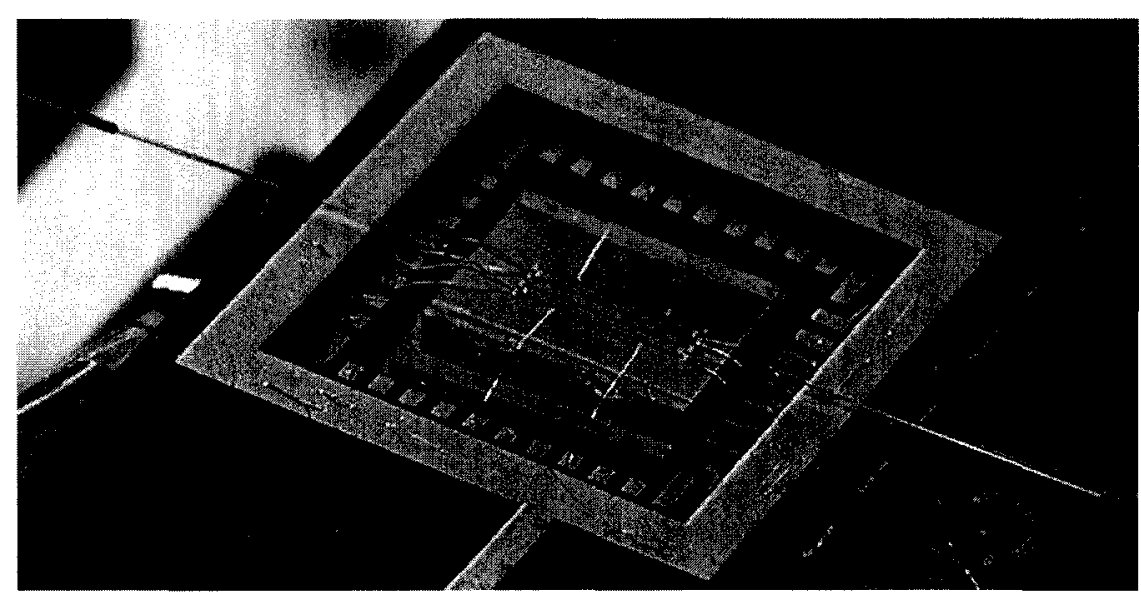

Figure 6.3. Packaged D1-3 device undergoing electrical testing. The capability exists for simultaneous optical characterisation.

The D1 device stream was not thermo-mechanically or mechano-optically characterised, but the experimental procedure developed for the electro-thermal characterisation was used for the D2 devices. The voltage/current relationship for a $2.0 \mathrm{~mm}, 1713 \Omega \mathrm{NiCr}$ heater on a D1 device is shown in figure 6.4 .

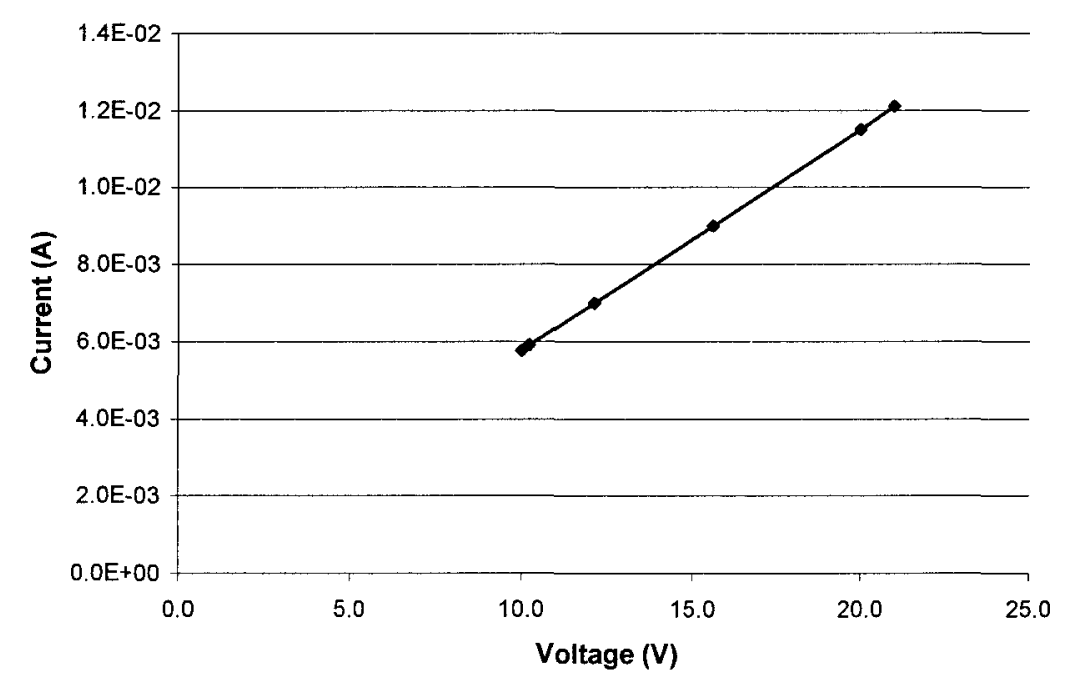

Figure 6.4. Voltage versus current for device D1-3. 


\subsubsection{D2 Device}

The device that was packaged for electro-thermal and thermo-optic characterisation was D2-22. This section will only describe the electro-thermal characterisation. As the current was increased through the resistive heater, the voltage across the element was measured and plotted in figure 6.6.

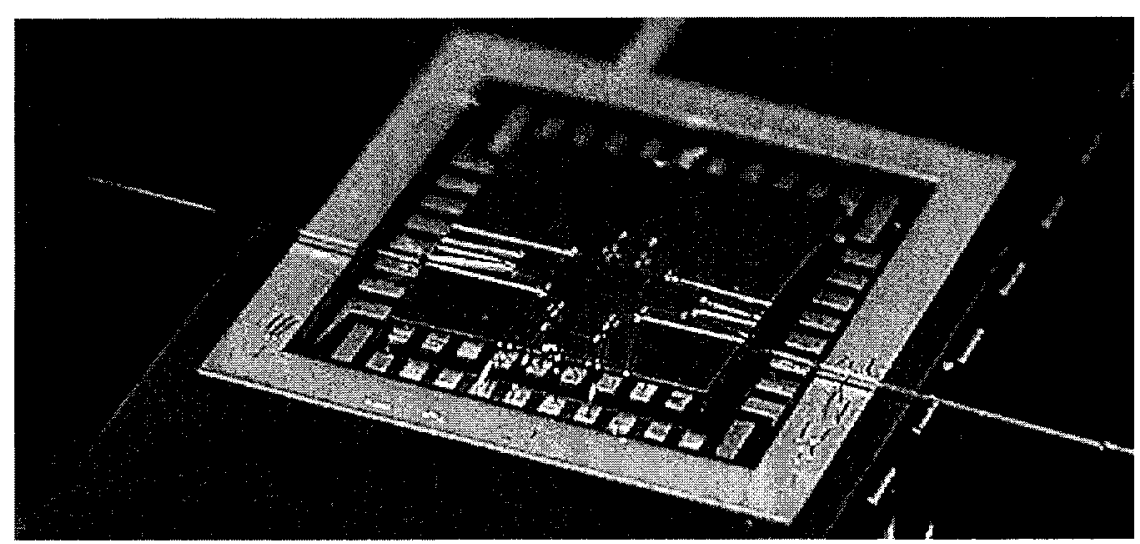

Figure 6.5. Packaged D2-22 device configured for simultaneous electro-thermal and thermo-optic characterisation.

The voltage and current values were in turn used to calculate the resistance, $R_{T}$, of the heater as its temperature, $T$, steadily increased. With these values, along with a TCR for gold of $3400 \mathrm{ppm}^{\circ} \mathrm{C}^{-1}$ [91], it was possible to use equation 3.15 to calculate the increase in temperature, $T$, as $R_{T}$ changed. At a room temperature, $T_{0}=22.0{ }^{\circ} \mathrm{C}$, the resistance of the heater, $R_{0}$ measured 8.0 $\Omega$. The experimental results are plotted in Figure 6.7 for the range of operation the device was tested over. The relative uncertainty of the calculated resistance, $R_{T}$, ranged from $\pm 0.08 \Omega$ to $\pm 0.03 \Omega$. The propagation of error through equation 3.15 to calculate heater temperature, $T$, resulted in a relative uncertainty that ranged from $\pm 4.8^{\circ} \mathrm{C}$ to $\pm 3.0^{\circ} \mathrm{C}$. 


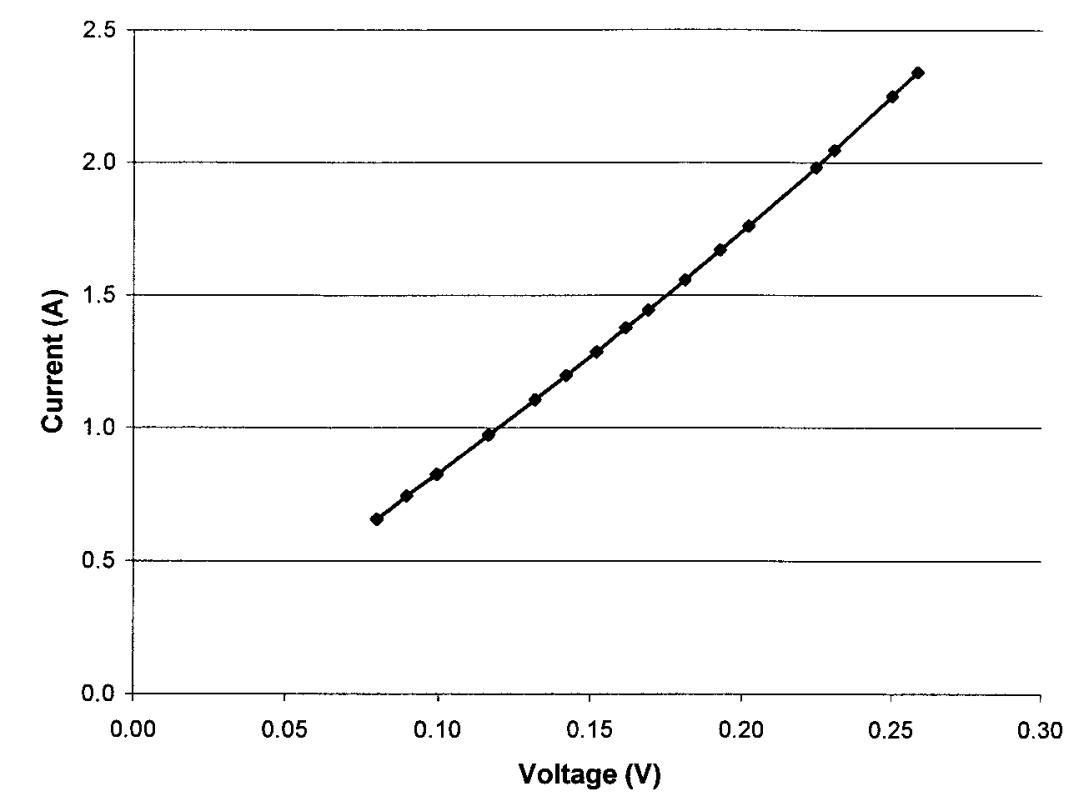

Figure 6.6. Voltage versus current for device D2-22.

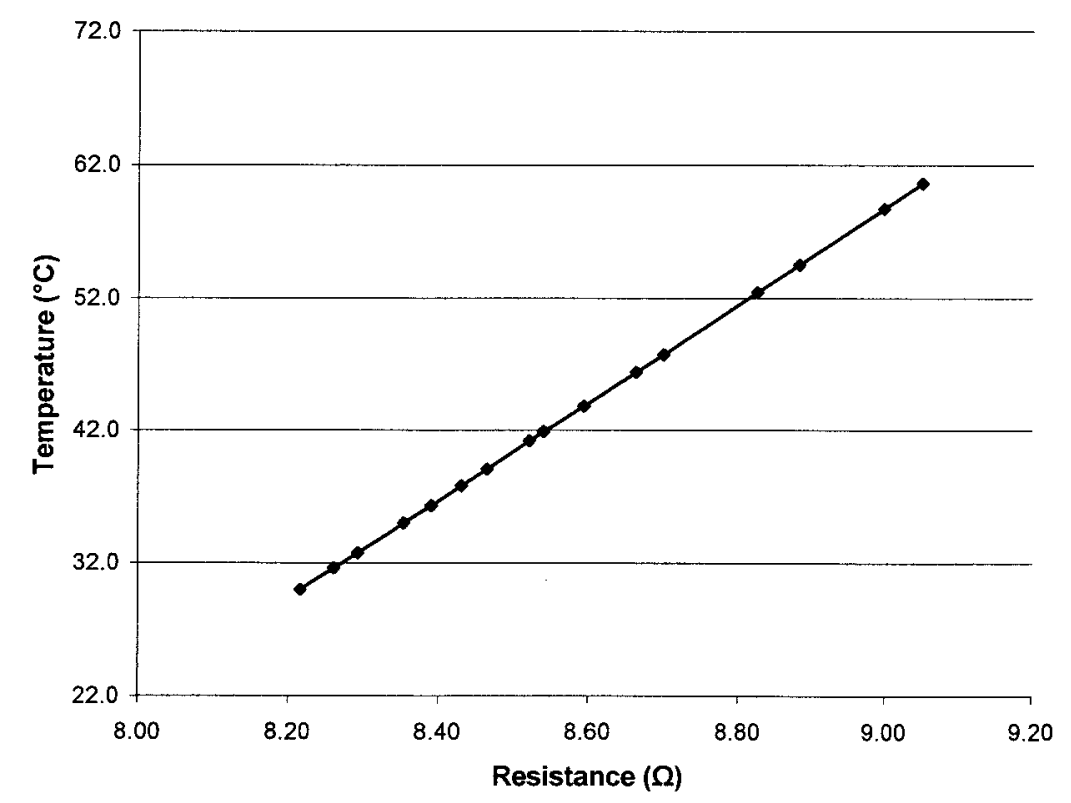

Figure 6.7. Calculated heater resistance versus the temperature calculated from the TCR of gold.

These heaters were integrated with unreleased waveguide bridge filters and 
thermo-optically characterised in section 6.3.2. The rate of the thermo-optical shift measured in this device was mapped to the thermo-optical shift measured from devices externally heated by the laser diode used in the thermomechano-optical characterisation.

\subsection{Thermo-mechanical Characterisation}

Thermo-mechanical characterisation of the released bridge waveguides on SOI was performed by an external heater on unpackaged die from the D2 stream. This method allowed rapid verification of a successfully released bridge out of the fabrication lab, as well as characterisation of devices that did not have an integrated heater.

Figure 6.8 depicts the thermo-mechanical experimental setup that was constructed at the Carleton University Photonics Lab. A Thorlabs L808P200 200mW, $808 \mathrm{~nm}$ laser diode (LD); controlled by a Thorlabs LDC500 laser diode controller (LDC); was focused onto a suspended bridge to heat it over its pre- to post-buckle regimes. Recalling figure 1.4 of the absorption coefficient for semiconductors, silicon is highly absorptive of light at this wavelength, making the LD ideal to heat the silicon waveguide bridge.

The laser diode, collimator, and microscope objective lens assembly was mounted as a unit on a goniometer so that a fixed angle of laser impingement could be maintained throughout the thermo-mechanical and thermomechano-optical experiments. The microscope lens chosen was a 10X Mitutoyo M Plan Apo objective with a working distance of $33.5 \mathrm{~mm}$ to allow clearance for the other test bench elements.

The laser heater assembly was deflected $45^{\circ}$ from the horizontal as shown in figure 6.8. At this angle, the focused laser spot was elongated to a $190 \mu \mathrm{m}$ line. The laser light is shown in figure 6.9, directed onto a smooth silicon surface in which test 
bridges without rib waveguides have been released.
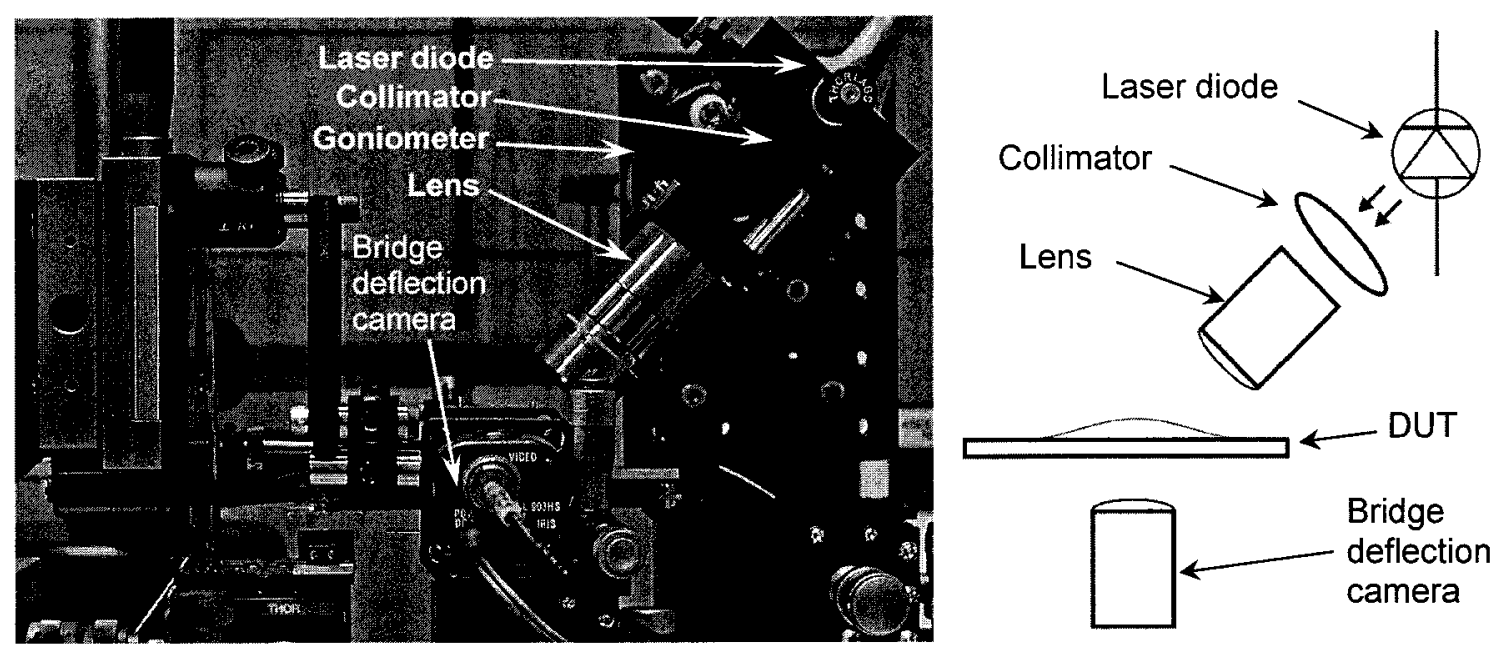

Figure 6.8. Test setup for external heating from a laser diode for thermo-mechanical bridge deflection characterisation.

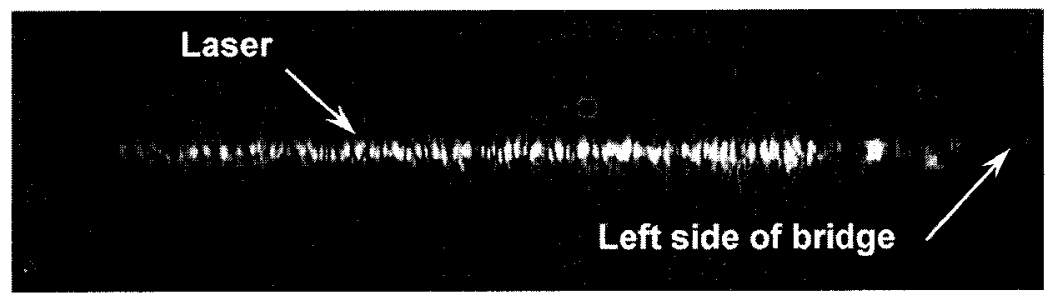

Figure 6.9. Image of laser directed onto a smooth silicon surface on which test bridges have been released (FOV $250 \mu \mathrm{m}$ ).

A series of power measurements were made to determine the threshold current of the laser, and the reversibility of the laser power as a function of drive current. The plot is shown in figure 6.10. It reveals that the linearity of the laser can be extended back to $98 \mathrm{~mA}$ of LD current, with a rate of $0.77 \mathrm{~mW} / \mathrm{mA}$. The product specification sheet for the L808P200 LD reports a typical slope efficiency of $0.7 \mathrm{~mW} / \mathrm{mA}$, and a maximum of $0.9 \mathrm{~mW} / \mathrm{mA}$. The reversibility of the laser power is evident as the LD drive current is cycled up and down to the same LD power at the same LD current.

A second series of measurements were made to examine the linearity of the LD output power over the range of operation used to thermally actuate the bridge structures. 
The plot shown in Figure 6.11 indicated stable linearity centred around the LD power a $2000 \mu \mathrm{m}$ bridge would buckle at.

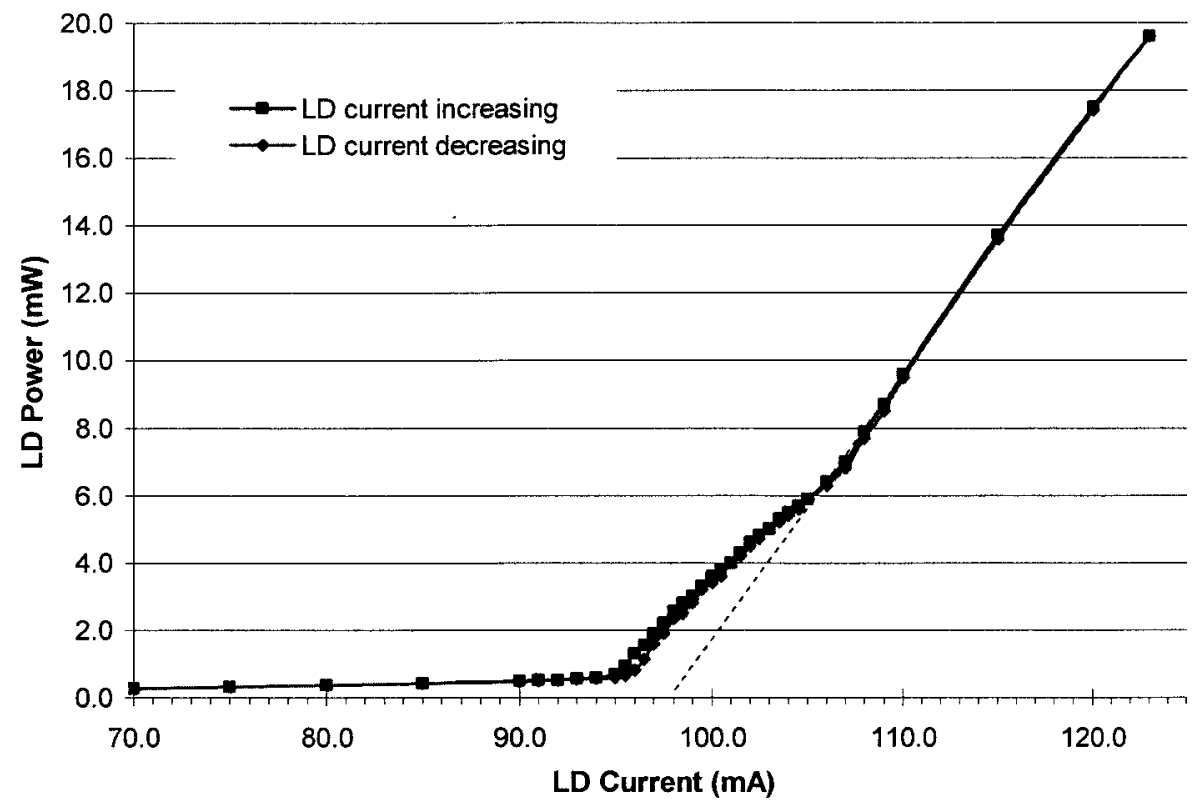

Figure 6.10. LD Drive Current versus LD Power, indicating reversibility in the LD current/power relationship.

In addition to the laser actuation setup described above, a secondary method of externally heating the waveguide bridge was assessed in anticipation of adapting the heater for packaging. The LD, collimator, and objective lens assembly were moved off the goniometer to the optical bench, where the laser light was coupled to a $20 \mu \mathrm{m}$ core fibre. The other end of the fibre was mounted back on the goniometer, allowing the fibre tip to be rotated into perpendicular alignment above the centre of the bridge, where it would project a $50 \mu \mathrm{m}$ diameter spot. This variation on the external heater assembly is shown in figure 6.12 . 


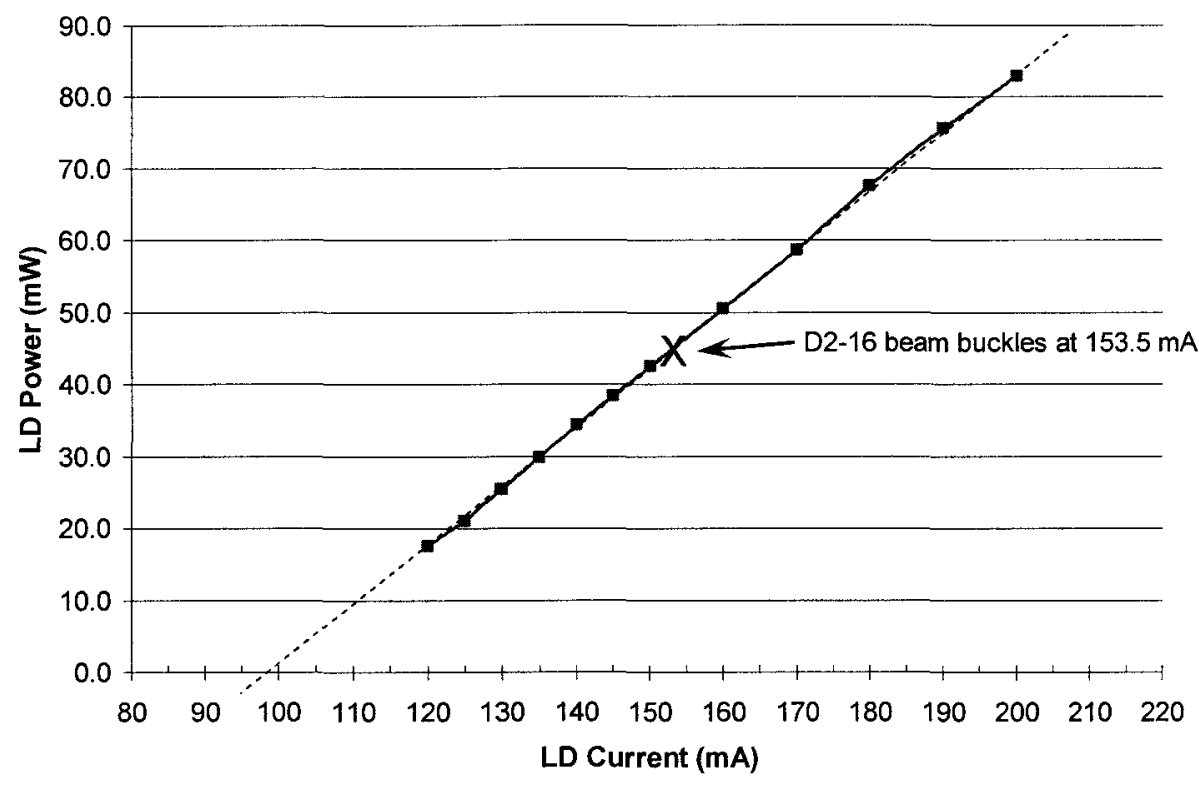

Figure 6.11. LD Drive Current versus LD Power plotted to show the linearity of the LD power over the range of thermo-mechanical characterisation. The LD current at which a $2000 \mu \mathrm{m}$ bridge buckles is labelled.

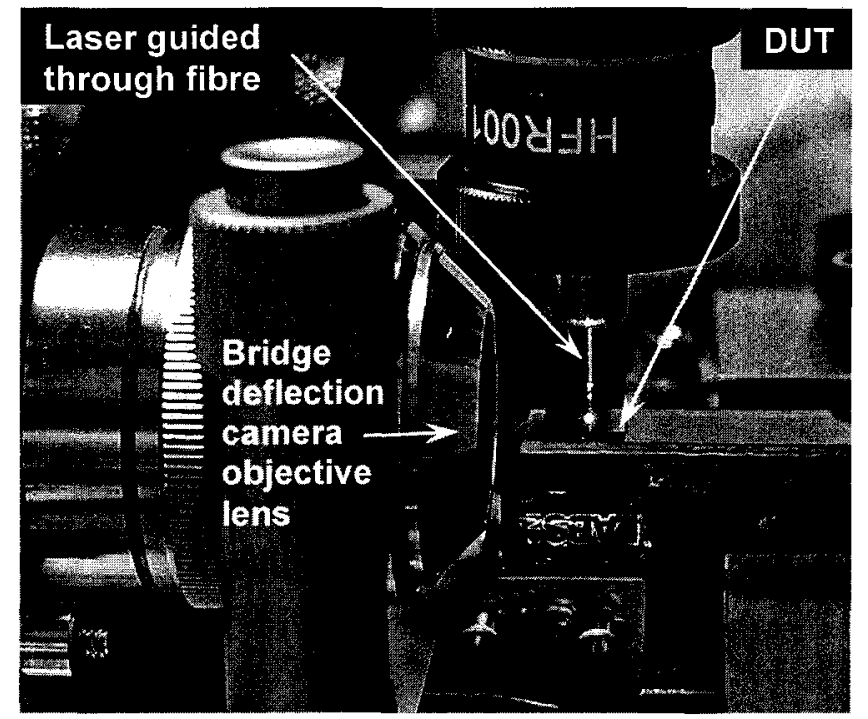

Figure 6.12. Alternative method of external heating by guiding the laser light directly to the bridge through a fibre. Also shown is the bridge deflection camera orientation to capture buckle height.

A Watec LCL-903HS CCD camera with $20 \mathrm{x}$ objective was used to image the waveguide bridge deflection. Illumination was directed in-plane, across the DUT, into 
the camera objective. This was important to ensure the illumination did not distort the image. Conversely, illumination directed off the plane allowed for a longer depth of focus when centring test elements, such as the laser, over the $30 \mu \mathrm{m}$ wide bridge.

The bridge deflection camera was positioned perpendicular to the surface of the DUT as shown in figure 6.12. The deflection height on still images was measured using the ruler function of Photoshop Elements. The ruler was calibrated to the diameter of the laser actuation fibre shown in figure 6.13. The fibre width measured 590.00 ruler divisions, and was specified to be $123 \mu \mathrm{m}$ wide, which established that one micron represented 0.208 divisions on the ruler. The measurement view was set to "actual pixels" to ensure the software would not skew the image.

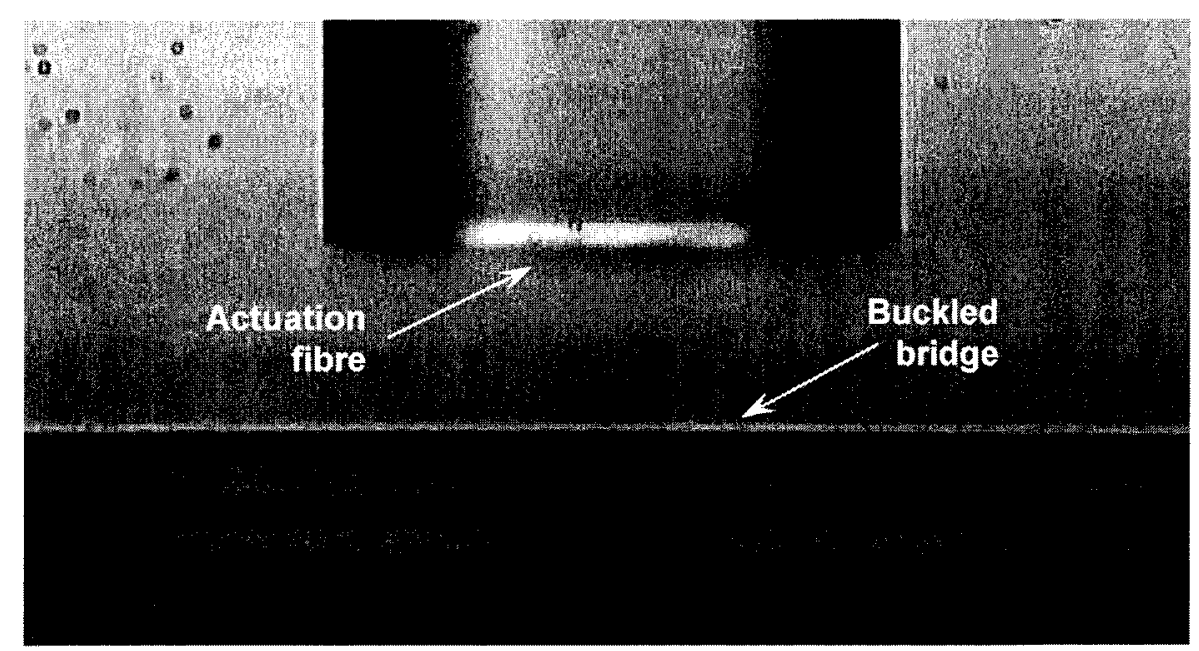

Figure 6.13. The $123 \mu \mathrm{m}$ width of the laser actuation fibre used to calibrate the ruler in Photoshop Elements for deflection height measurements (FOV $250 \mu \mathrm{m}$ ).

To measure the vertical bridge deflection based on a horizontal width, the aspect ratio of the image captured by the bridge deflection camera could not be distorted. A planar image of a mask alignment cross from the Bragg grating etch was captured by the bridge deflection camera, as shown in figure 6.14a. 220.50 horizontal ruler divisions were measured against 219.75 vertical ruler divisions for an aspect ratio of 1.00 to 1 . 
Using 0.208 divisions/micron, the cross measured $45.9 \mu \mathrm{m} \times 45.7 \mu \mathrm{m}$. The ruler conversion factor was validated by measuring the same cross in the fabrication lab using the line width metrology system, with a result of $45.5 \mu \mathrm{m} \times 45.3 \mu \mathrm{m}$, as shown in figure $6.14 \mathrm{~b}$.
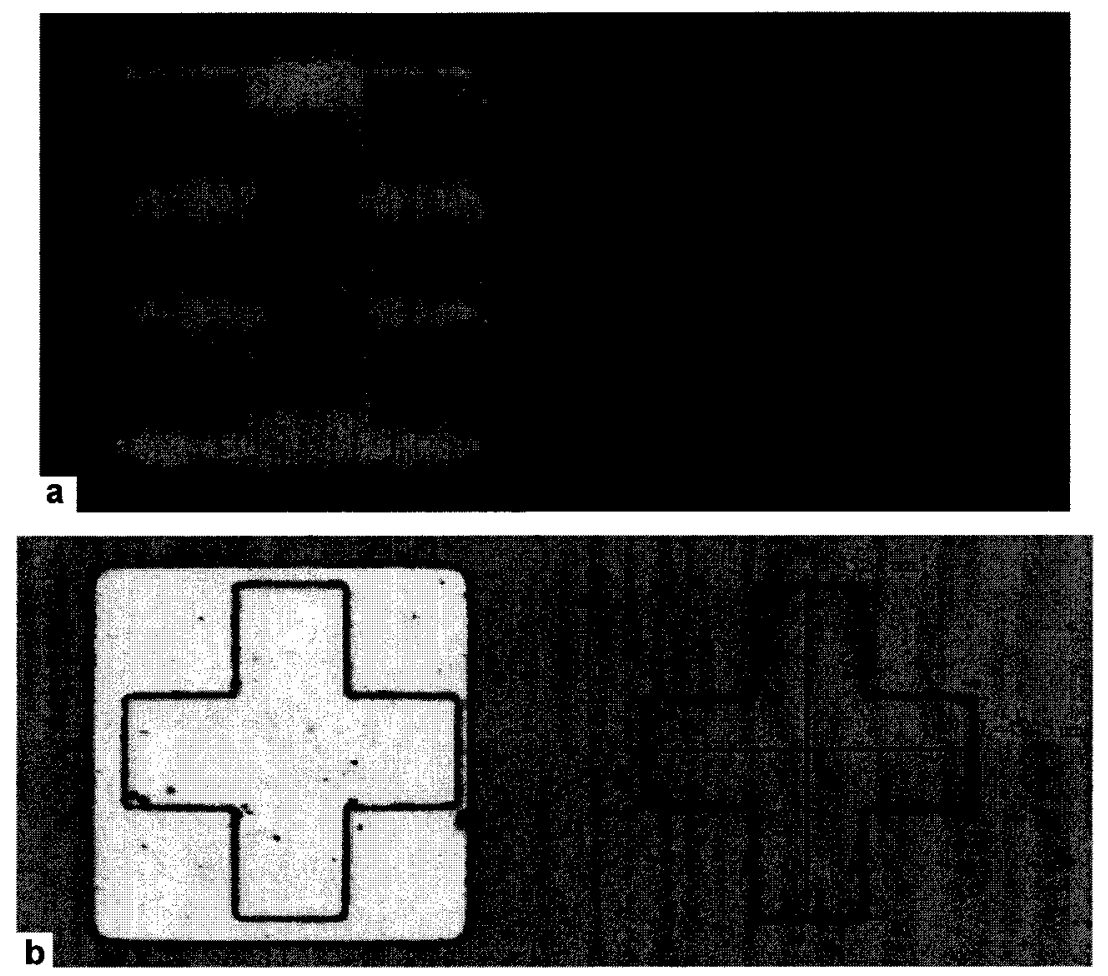

Figure 6.14. a) Mask alignment cross structure captured by the bridge deflection camera for pixel aspect ratio verification. Cross on the left is enclosed by gold deposition from heater mask alignment square, cross on the right is bare silicon. b) Sample line width measurement on the same structure from line width metrology system in fabrication lab.

This completed the calibration and verification of the thermo-mechanical experimental setup. To thermally actuate the suspended waveguide bridge, the LD current was increased over the linear region of laser output power. This resulted in a linear change in the temperature of the suspended waveguide bridge due to laser heating. The photomicrograph in figure 6.15 depicts a laser that is heating a $2000 \mu \mathrm{m}$ long, $5.0 \mu \mathrm{m}$ thick, $30 \mu \mathrm{m}$ wide bridge in the post-buckle regime. In order to take deflection 
measurements, an $800 \mathrm{~nm}$ filter was used to block the laser light to the bridge deflection camera. The camera was focused on the line defined by the contrast between the illuminated surface of the bridge and the unilluminated edge facing the camera.

Still images of the beam deflection were captured in $1.0 \mathrm{~mA}$ increments of LD drive current using the LDC. The drive current from the LDC was also regulated by an Interstate F74 sweep function generator, which produced a ramp sawtooth signal to steadily heat the bridge up and down over its range of operation for video capture. Still image and video inspection were conducted on $500 \mu \mathrm{m}, 1000 \mu \mathrm{m}$, and $2000 \mu \mathrm{m}$ bridges with both rib waveguide and rectangular cross-sections.
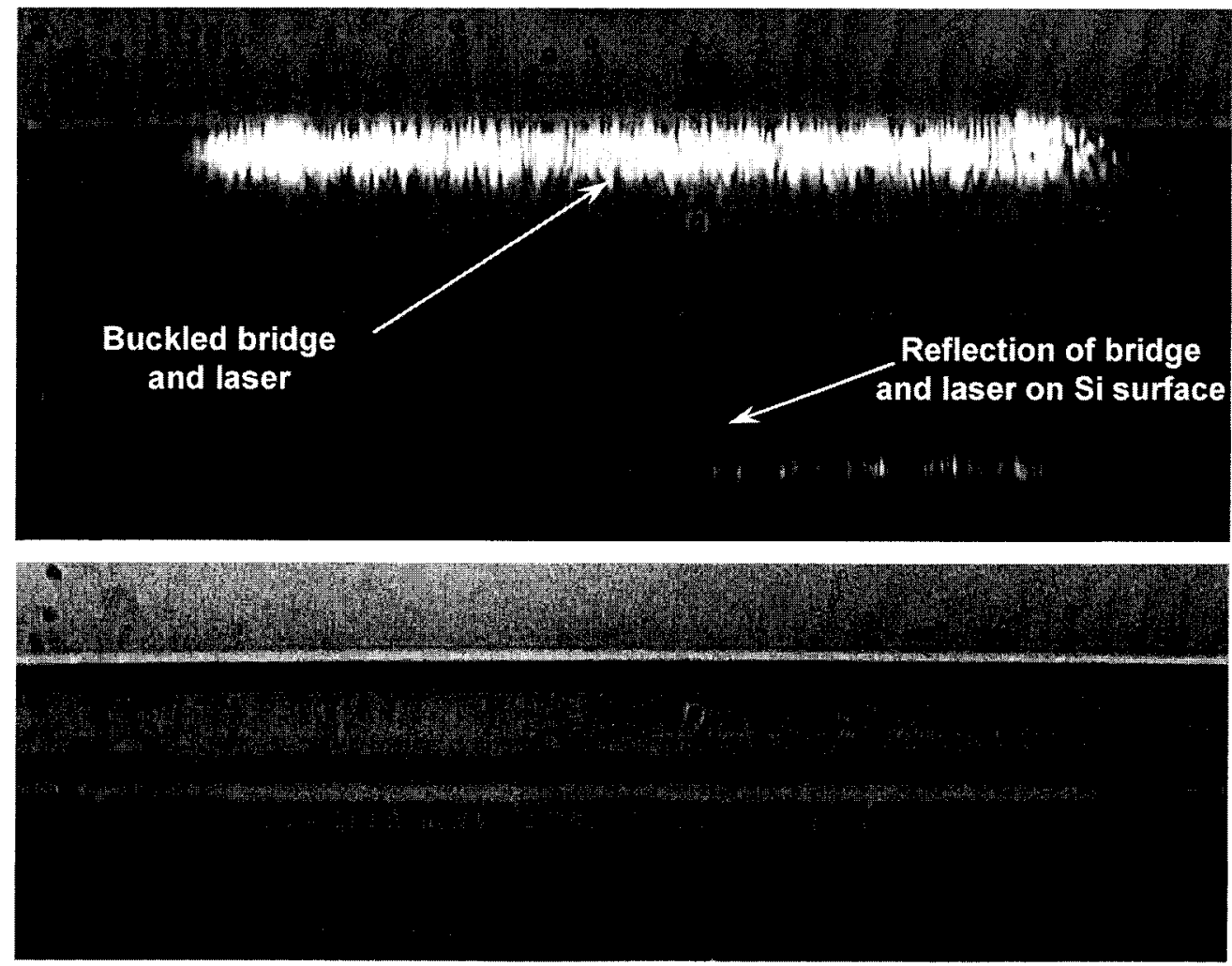

Figure 6.15. Photomicrographs of laser heating deflecting a $2000 \mu \mathrm{m}$ long, $5.0 \mu \mathrm{m}$ thick, $30 \mu \mathrm{m}$ wide bridge into the post-buckle regime without and with an $800 \mathrm{~nm}$ laser light filter $(\mathrm{FOV}=250 \mu \mathrm{m})$.

Experiments revealed different deflection behaviour for bridges of different cross- 
sections. Three types of deflection behaviour were observed for a rib waveguide bridge undergoing a continuous increase in thermal actuation: bumping, buckling, and bending. Although the term "buckle" is generally used to describe the initial movement of a thin film out-of-plane, these three terms are needed to differentiate between the observed behaviour of the rib waveguide bridge within the pre- to post-buckle regimes of thermal actuation.

A bridge that snaps abruptly out-of-plane to a height that is greater than the thickness of the bridge itself is defined to have buckled to its deflection height. A bridge that snaps abruptly out-of-plane to a height that is a fraction of the bridge thickness is defined to have bumped to its deflection height. A bridge that moves steadily out-ofplane without abrupt movement, and is in a continuous process of deflection, is defined to be bending. While the purpose of this thesis is not a detailed treatment of the mechanical behaviour of buckling beams, some important patterns were observed that give insight into the thermo-mechanical and thermomechano-optical characterisation that is to follow.

Bumping behaviour was only observed on bridges that had a rib waveguide. Test bridges that were rectangular in cross-section would only exhibit buckle or bending deflection. In cases where bump behaviour occurred, this small deflection initially remained fixed, even as the temperature continued to increase. After an increase of up to $12.0 \mathrm{~mA}$ of LD current from the initial bump (D2-20), the bridge would eventually exhibit buckle behaviour. An actuated rib waveguide bridge would return to the prebuckle regime at the same LD current (or temperature), whether the bridge had been actuated through the bump deflection into the post-buckle regime, or if it had only been actuated up to the bump deflection before returning to the pre-buckle regime. 
A bridge that began its actuation by bending out-of-plane would always eventually buckle to its mode shape, which was the beginning of the post-buckle regime. There it would return to bending steadily out-of-plane as the temperature continued to increase. If a bridge initially buckled to its mode shape, it would also continue to bend steadily out-of-plane as the temperature increased. The buckle height was the deflection height, $\delta$, of the stable mode shape when the bridge had reached its critical axial buckling pressure due to thermal expansion. This is the value described by equation 3.53 introduced in section 3.8

$$
\delta=\sqrt{\frac{d x 4 L_{0}}{\pi^{2}}}
$$

Figure 6.16 and figure 6.17 show suspended waveguide bridges in their postbuckle regime. It was difficult to photomicrographically capture the buckle shape of the $2000 \mu \mathrm{m}$ and $4000 \mu \mathrm{m}$ bridges due to the large length to thickness aspect ratio of up to 800:1. The shortest bridge, at $500 \mu \mathrm{m}$, could be captured in a single frame, while a $2000 \mu \mathrm{m}$ long bridge required a photomosaic to capture the buckle shape. The SEM would be an ideal tool to capture the buckling mode shape and subsequent post-buckle deflection height, but there were no means available to actuate the bridges inside the imaging chamber.

Bridge deflection measurements at the critical buckling temperature were made experimentally using the techniques described in this section, and compared to the theoretical values using the method of analysis developed in chapter 4. The comparison was conducted for both the rib and rectangular bridge cross-sections of $500 \mu \mathrm{m}$, $1000 \mu \mathrm{m}$, and $2000 \mu \mathrm{m}$ lengths. The results are plotted in figure 6.18 and figure 6.19 . 
The theoretical buckle heights were in agreement with the measured buckle heights. This confirmed that the experimental buckle height measurements represented the deflection of the beams to their buckling modes.

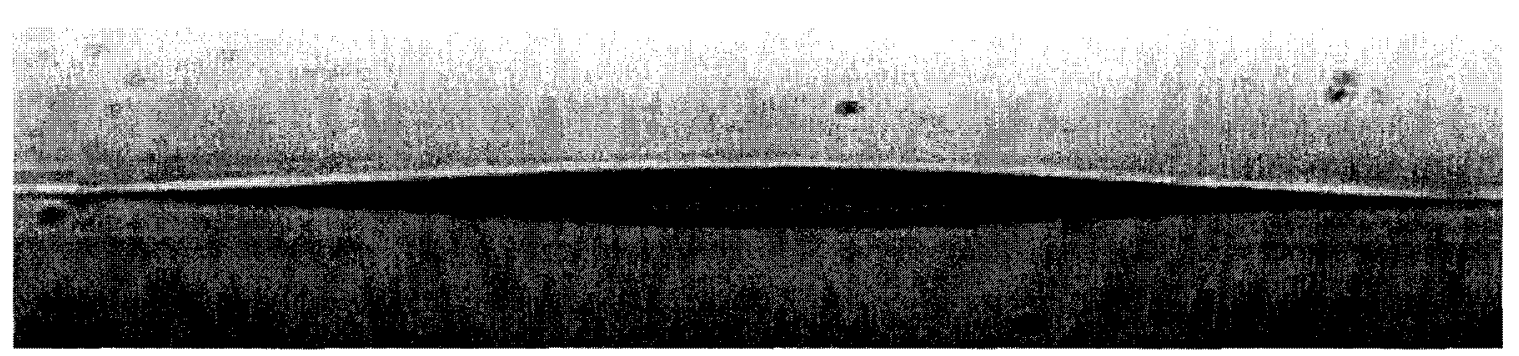

Figure 6.16. $500 \mu \mathrm{m}$ long, $890 \mathrm{~nm}$ deep rib waveguide bridge in post-buckle regime (D2-16 WG-A).

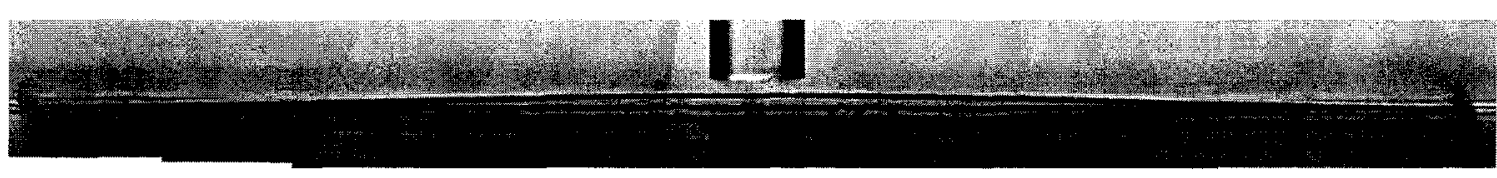

Figure 6.17. $2000 \mu \mathrm{m}$ long, $890 \mathrm{~nm}$ deep rib waveguide bridge in post-buckle regime using the $123 \mu \mathrm{m}$ wide thermal actuation fibre (D2-16 WG-1).

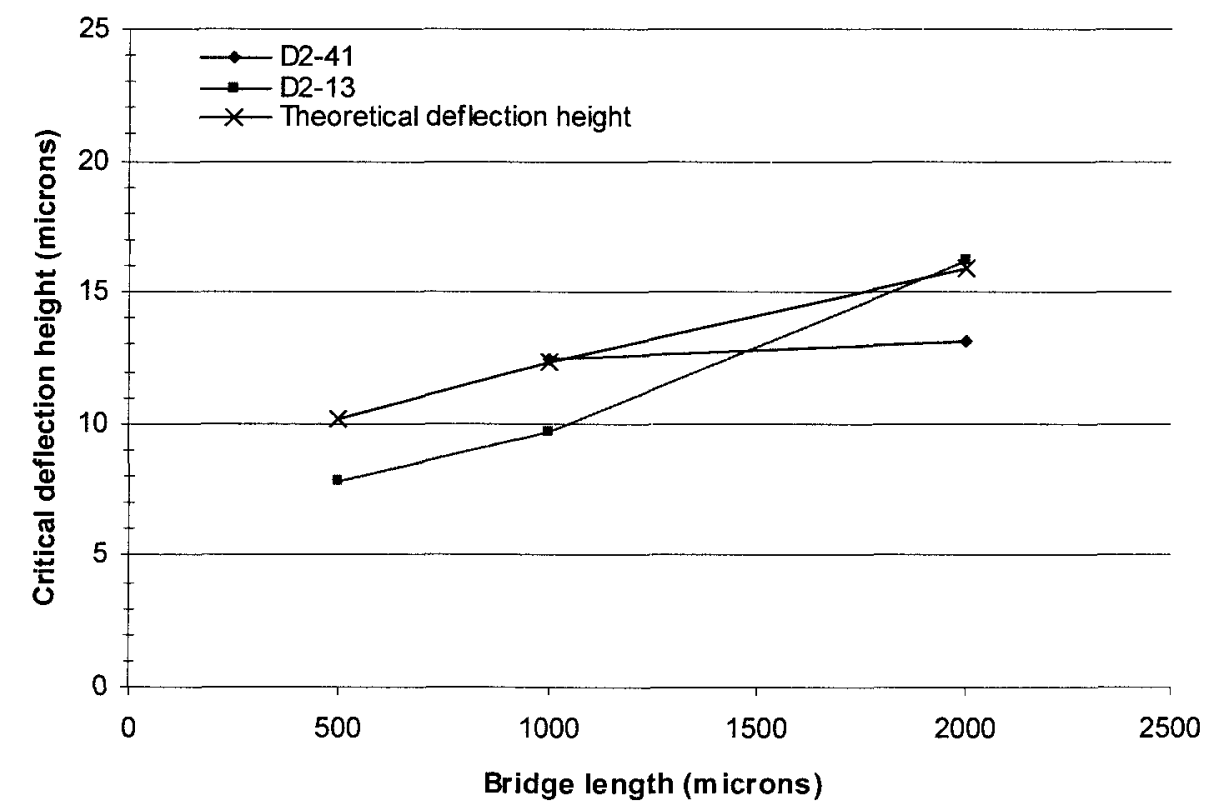

Figure 6.18. Critical buckle height versus bridge length for a $30.9 \mu \mathrm{m}$ wide, $5 \mu \mathrm{m}$ thick rectangular cross-section bridge. 


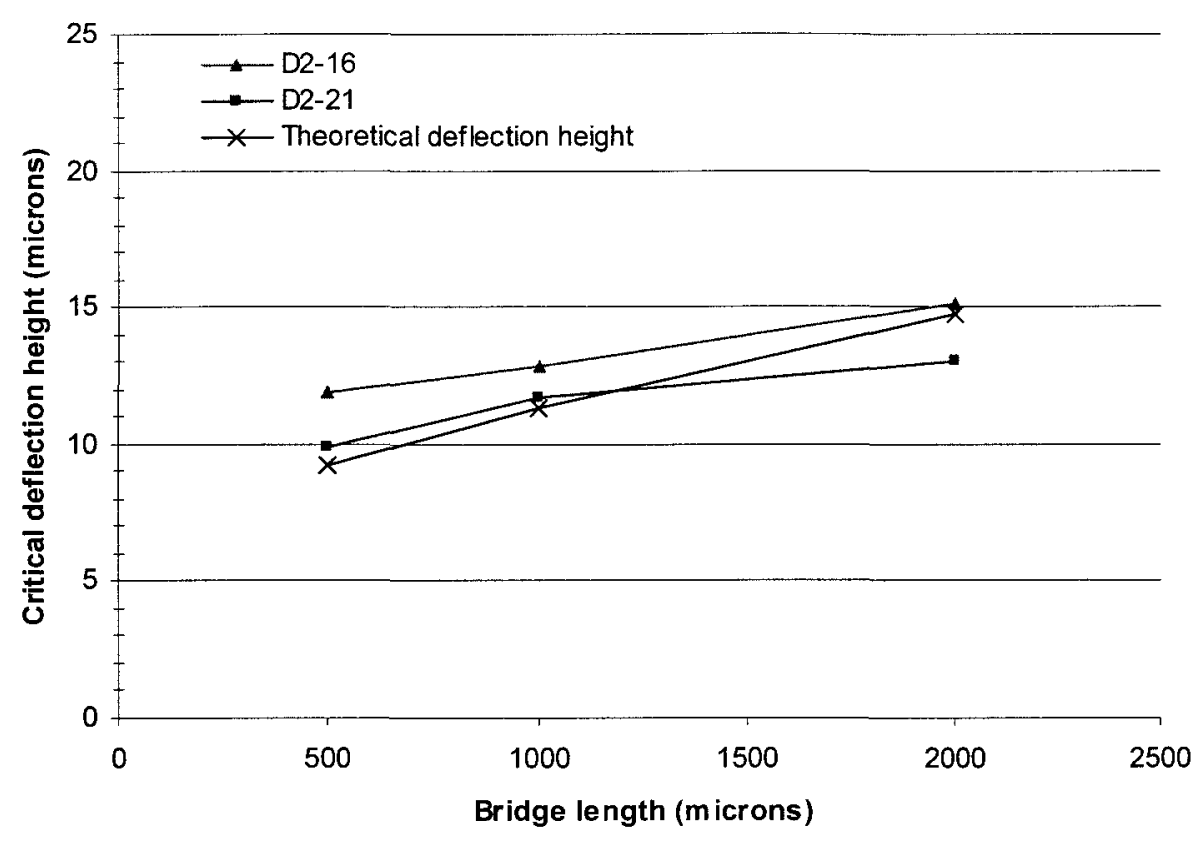

Figure 6.19. Critical buckle height versus bridge length for a $30.7 \mu \mathrm{m}$ wide, $890 \mathrm{~nm}$ deep rib waveguide bridge.

The next experimental series conducted was a detailed deflection analysis over the entire pre- to post-buckle range of a specific rib waveguide bridge with integrated Bragg filter. The device chosen for this detailed thermo-mechanical analysis would also undergo thermomechano-optical characterisation. For a device to undergo the full range of characterisation via external heating, there were four major gates in the fabrication process flow that had to be completed successfully. This included a grating and waveguide etch that produced a strong enough Bragg dip to measure through-out the entire range of operation; two facet cleaves that would allow an optical signal to be coupled into and detected from the waveguide; and a successful bridge release etch. Failure at any of these gates would render the device inoperable.

The die could also be rendered inoperable in the photonics lab by contamination. In one instance of contamination, a dust particle settled on the centre of a rib waveguide 
bridge (D2-16 WG-3) that was initially chosen for the full range of characterisation. The laser that thermally actuated the waveguide bridge fused the particle to the bridge surface, which changed its buckle characteristics and rendered the device useless for experimentation. To help increase the likelihood of producing a fully testable device on a given die, the D2 mask design in section 4.5 provided three instances of a device for each bridge length on each die. D2-16 possessed the best fabrication results from the main process gates. Therefore the full thermo-mechanical and thermomechano-optical range of experimental analysis was conducted on D2-16 WG-1, which contained a $1000 \mu \mathrm{m}$ Bragg grating filter integrated on a $2000 \mu \mathrm{m}$ rib waveguide bridge.

The detailed thermo-mechanical characterisation of the beam deflection behaviour was conducted in $1.0 \mathrm{~mA} \mathrm{LD}$ current increments. With these small current increments the occurrence of the bump, buckle and bending behaviour could be clearly observed. An initial bump deflection height of $0.5 \mu \mathrm{m}$ occurred between $144.0 \mathrm{~mA}$ and $145.0 \mathrm{~mA}$ of LD current. This height remained unchanged for a further increase of $8.5 \mathrm{~mA}$ in LD current. Subsequently the bridge deflected to its buckling mode height of $15.1 \mu \mathrm{m}$ at a LD current between $153.0 \mathrm{~mA}$ and $153.5 \mathrm{~mA}$. In the post-buckle regime the bridge continued to steadily bend out-of-plane. These definitive bridge deflection heights, along with the temperature they occurred at, are shown in figure 6.20. Although thermal actuation is shown as a function of LD current in this section, section 6.3 .2 will outline how temperature values were derived from the thermo-optical response of an integrated heater. 

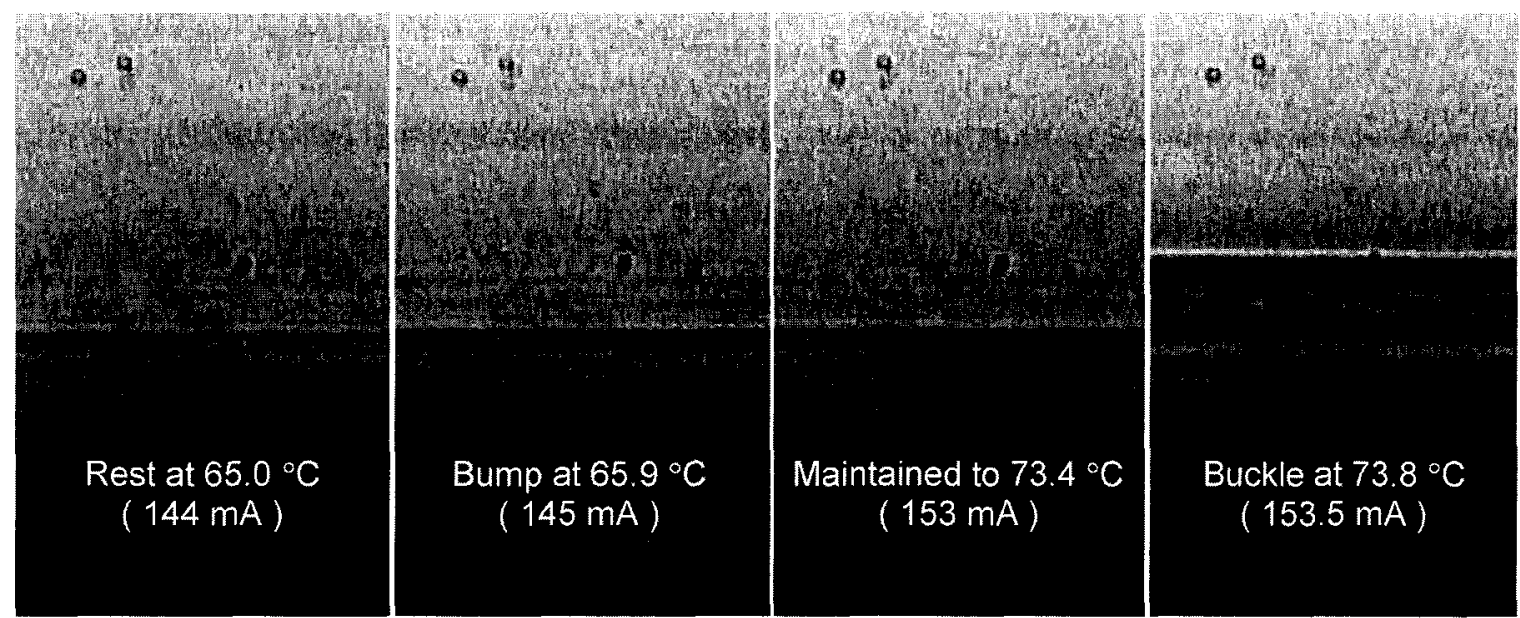

Figure 6.20. $2000 \mu \mathrm{m}$ long, $890 \mathrm{~nm}$ deep rib waveguide bridge at rest, $0.5 \mu \mathrm{m}$ bump deflection, and $15.1 \mu \mathrm{m}$ buckle deflection heights (D2-16 WG-1).

When the LD current was decreased in the post-buckle regime, the bridge deflection height decreased along the same path that the deflection height increased. As the LD current was reduced to $153.0 \mathrm{~mA}$, the bridge remained in the post-buckle regime, as its deflection height continued to steadily decrease. The bridge began to move back to its pre-buckle configuration in greater deflection increments between $141.0 \mathrm{~mA}$ and $140.0 \mathrm{~mA}$, until $138.0 \mathrm{~mA}$ to $137.0 \mathrm{~m}$; where the bridge returned to its in-plane prebuckle regime. The roll-off in bridge deflection height that occurred between $141.0 \mathrm{~mA}$ and $137.0 \mathrm{~mA}$ is typical behaviour for a deflected beam or plate that is incrementally returning to its rest state.

In the realm of MEMS devices, bi-stable behaviour is usually considered to have occurred if a structure can rest in either of two different deflection states without consuming power [78]. If the necessity for power is neglected, the rib waveguide bridge can be considered bi-stable between $138 \mathrm{~mA}$ and $153.0 \mathrm{~mA}$ of LD current. While this bistability is only in effect while the beam is being heated, it will have implications for the optical performance of the device later in the characterisation process. 
The bridge deflection height measurement as a function of LD current over the pre- to post-buckle regimes, as well as the return path to the pre-buckle regime, is plotted in figure 6.21. The next section, 6.3, will describe the thermo-optical and thermomechano-optical characterisation that will be conducted on the same $2000 \mu \mathrm{m}$ suspended rib waveguide filter, D2-16 WG-1. The transformation of LD current to bridge temperature will be accomplished in section 6.3 .2 by comparing the thermo-optic sensitivity of a LD heated device with an integrated heater device.

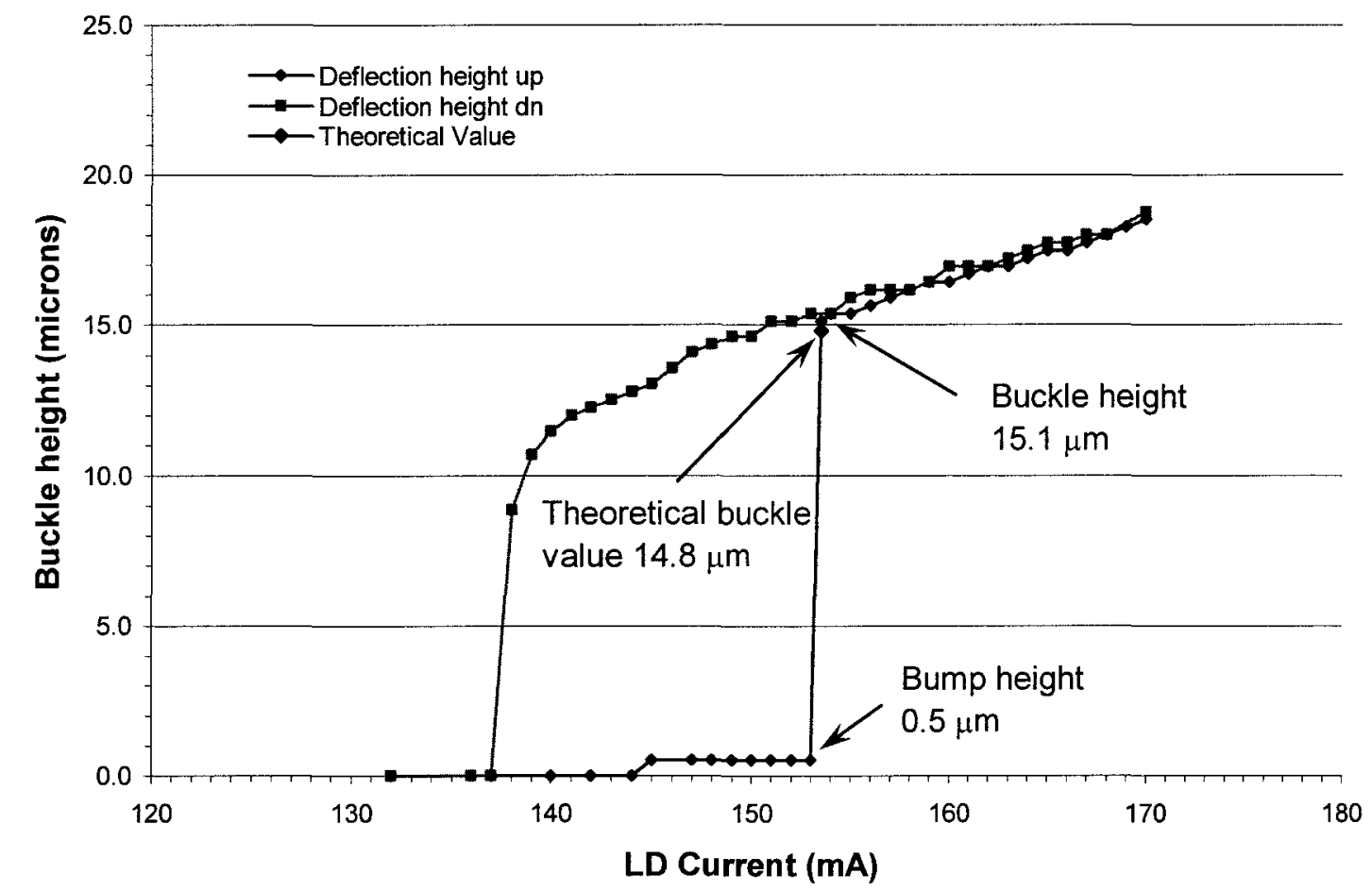

Figure 6.21. Deflection height versus LD Current for D2-16 WG-1. 


\subsection{Thermo-optical and Thermomechano-optical Characterisation}

\subsubsection{Optical characterisation}

This section will describe the optical characterisation that was performed on the proof of concept PC die. These measurements were made at IMS-NRC to validate the room temperature Bragg response for a Bragg filter waveguide on SOI. A spectral analysis was performed on the TE transmission spectrum of the device, since the Bragg response was stronger in this polarisation.

The test setup included a tunable laser that would step discretely through the spectrum of interest in $0.2 \mathrm{~nm}$ increments, at $1 \mathrm{~mW}$ of optical power. The output optical power from the Bragg grating waveguide was measured by an opto-electronic detector and converted back to an optical power response. Figure 6.22 depicts the test setup in which a lensed fibre would input the optical signal, and a microscope objective would gather the output light for the detector. The die tested was PC-1, which featured a rib height of $660 \mathrm{~nm}$, a rib width of $2.8 \mu \mathrm{m}$, and a grating depth of $210 \mathrm{~nm}$.

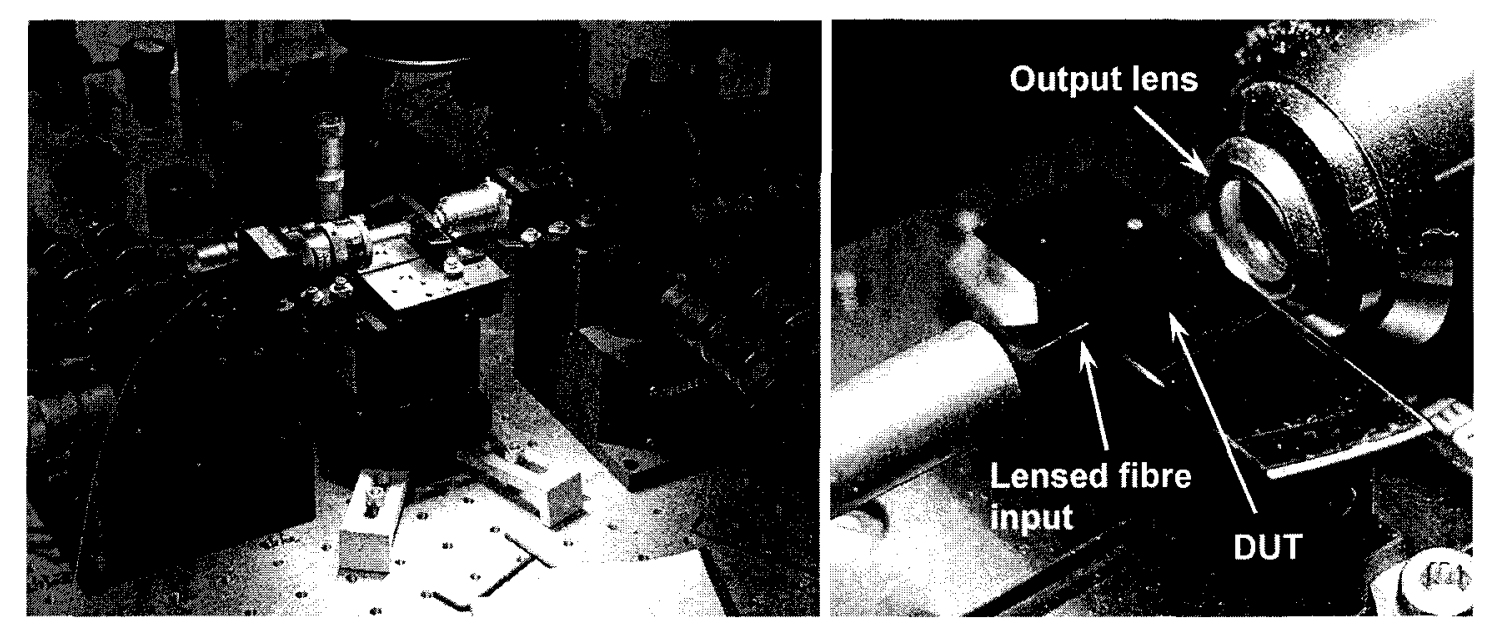

Figure 6.22. IMS-NRC Optical test bench with the PC-1 test die in place for characterisation.

The raw spectral data was reduced to graphical format by normalizing the output 
power to $\mathrm{dBm}$ for the tunable laser diode transmission wavelength. The resulting spectrum for a $1.0 \mathrm{~mm}$ grating is shown in figure 6.23 . The fundamental mode of the reflection wavelength, $\lambda_{B}$, was measured at $1555.6 \mathrm{~nm}$. The higher order leaky mode dips in the spectral response resulted from the forward fundamental mode coupling with the subsequent backward travelling higher order leaky modes.

These findings agreed with results published by T. Murphy et al. [115] for a Bragg filter on SOI rib waveguide, shown in figure 6.24. Using SOI with a $3 \mu \mathrm{m}$ device layer; the $1543 \mathrm{~nm}$ fundamental Bragg dip was generated via a $4000 \mu \mathrm{m}$ long grating with a pitch of $223 \mathrm{~nm}$ and a depth of $150 \mathrm{~nm}$; on a $4 \mu \mathrm{m}$ wide, $800 \mathrm{~nm}$ deep rib waveguide.

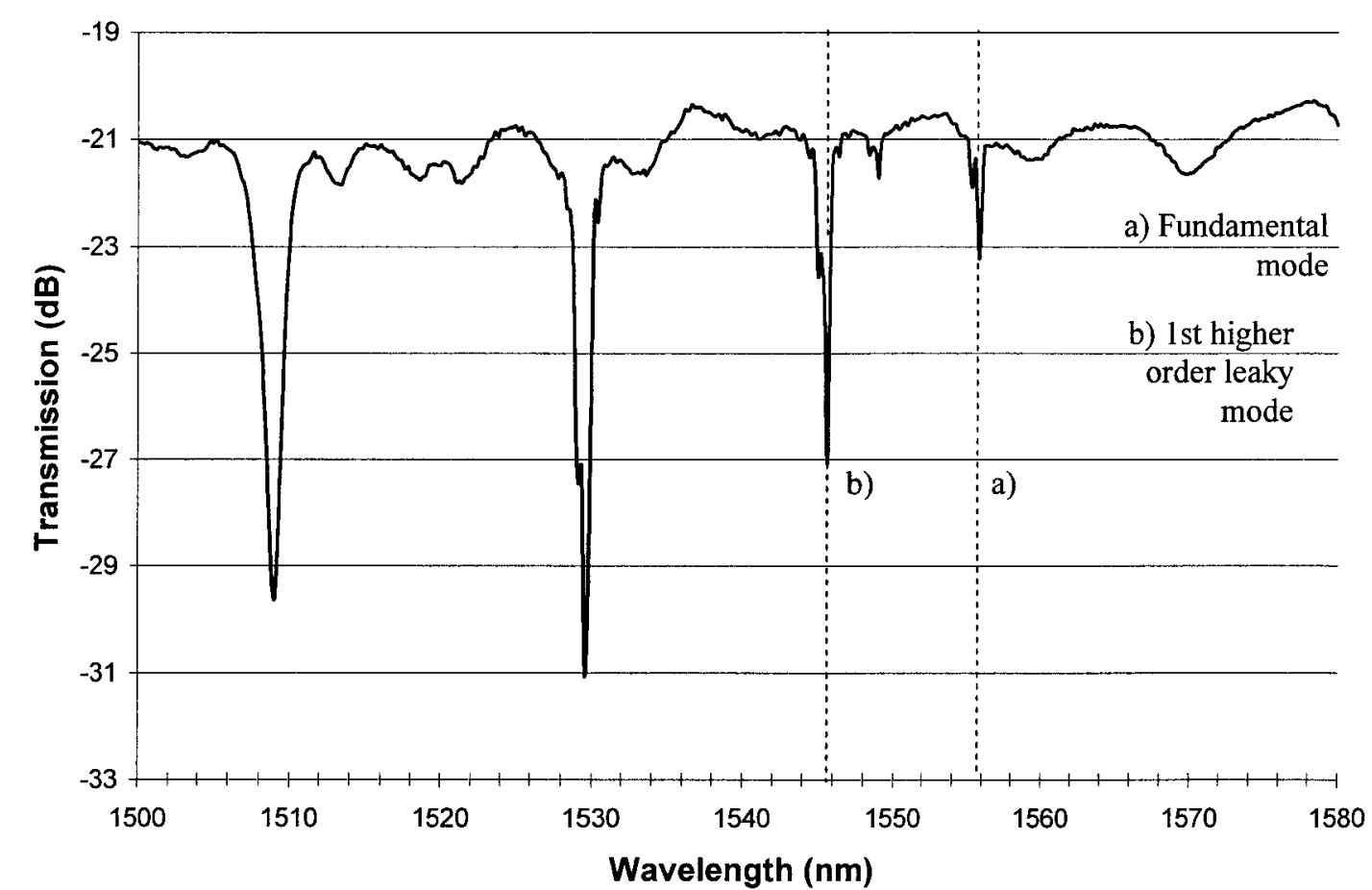

Figure 6.23. Transmission spectrum for PC-1 along a $1.0 \mathrm{~mm}$ Bragg grating. The fundamental mode of the Bragg wavelength at $1555.6 \mathrm{~nm}$ is shown along with three higher order leaky mode wavelengths. 


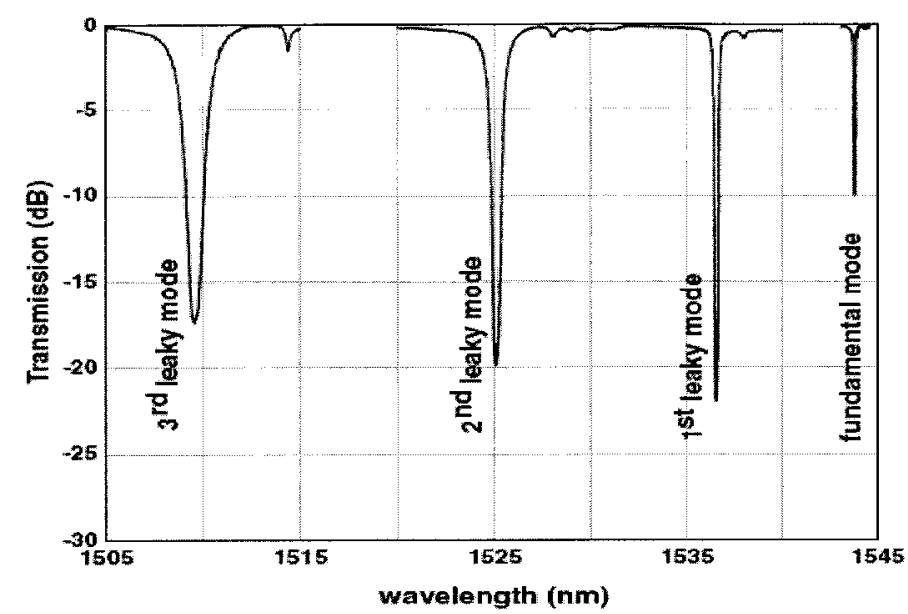

Figure 6.24. Results from 4mm Bragg grating published by T. Murphy [115]

After the optical behaviour of the PC device was established for a Bragg grating filter on an SOI rib waveguide, an optical test bench was assembled to conduct the thermo-optical and thermomechano-optical characterisation of the D1 and D2 device streams. This optical test bench was set up at Carleton University's Photonics Lab and integrated with the thermo-mechanical test bench described in section 6.2. While the optical test capability of this apparatus would not have the same functionality as the optical test bench at IMS-NRC, it would nevertheless ensure continuity between the thermo-mechanical measurements, and the thermo-optical and thermomechano-optical measurements to follow.

The main elements of the optical test bench had already been assembled and tested by Khaled Mnaymneh for use with his work on the optical characterisation of quasi-crystal devices [95]. The test bench was configured with a tunable laser and nearIR detector for fibre alignment to the waveguide facets, and a Teem Photonics supercontinuum (SC) unpolarised broad band light source and optical spectrum analyser (OSA) for the spectral analysis of the Bragg filter. 
The optical power provided by the $\mathrm{SC}$ at a specific wavelength would be low in comparison to the single wavelength of the tunable laser. Therefore the output signal would be closer to the noise level, making characterisation more difficult. The advantage was that the simultaneous emission of the entire spectrum of wavelengths allowed for rapid and straightforward characterisation of the device performance.

While the spectrum of wavelengths over which the device was tested ranged from 1300 to $1600 \mathrm{~nm}$, the span generated by the SC, at the same power, ranged back to $600 \mathrm{~nm}$. Silicon has relatively high absorption in the $600 \mathrm{~nm}$ to $900 \mathrm{~nm}$ range. Consequently the same material property that made the $808 \mathrm{~nm}$ laser diode a good candidate to heat the waveguide bridges for buckle experiments would also heat the input facet of the waveguide. Without protection from this range of wavelengths, the light focused on the silicon facet by the lensed input fibre was enough to ablate the material from the surface, rendering the waveguide facet useless. The solution was to insert a $1100 \mathrm{~nm}$ edgepass filter into the optical path of the SC. This was accomplished through the use of a fibre optic U-Bench shown in Figure 6.25.

The fibre carrying the SC signal was mounted on a three axis stage for alignment to the input facet of the DUT waveguide. A Corning OptiFocus lensed fibre was used to increase the coupling efficiency into the waveguide. The fibre itself is a single mode SMF-28 fibre, while the lensed taper provides a $3.3 \mu \mathrm{m}$ spot size at the beam waist. An overhead stereo microscope was used for course fibre to waveguide alignment. To enable fine alignment a tunable laser source was used to launch a beam, from the same input fibre, into the waveguide at $1550 \mathrm{~nm}$ with a power of $1 \mathrm{~mW}$. A near-IR sensitive camera, coupled to a $20 \mathrm{x}$ objective lens, was focused on the output facet of the DUT 
waveguide to detect when the input fibre was properly aligned to its waveguide facet.

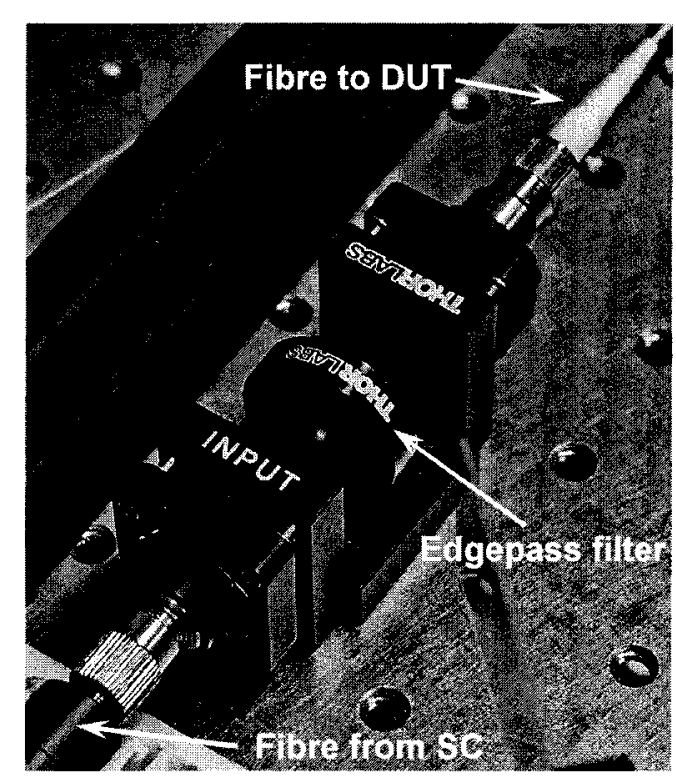

Figure 6.25. Fibre optic U-bench used to allow the insertion of a $1100 \mathrm{~nm}$ edgepass filter into the optical path of the SC source.

Both the output fibre and the near-IR camera were mounted on a translational base, which was itself mounted on a three axis stage. Once the camera confirmed that light was properly coupled to the input facet from the source, the translational stage allowed the output fibre to replace the camera. The output fibre was connected to an HP 70950 OSA. The signal strength measured by the OSA was used to fine tune the overall alignment of the input and output fibres to their respective waveguide facets.

Figure 6.26 depicts a schematic of the integrated thermo-mechanical and thermomechano-optical test bench that was assembled, while figure 6.27 pictures the complete setup, including the SC source. The box outline within figure 6.27 indicates a close-up of the test bench, shown in figure 6.28. This includes the DUT, the test stage illumination, the input fibre from the SC or tunable laser source, the external laser heater, the bridge deflection camera, the output fibre to the OSA, the near-IR camera, and the microscope for coarse fibre to waveguide alignment. 


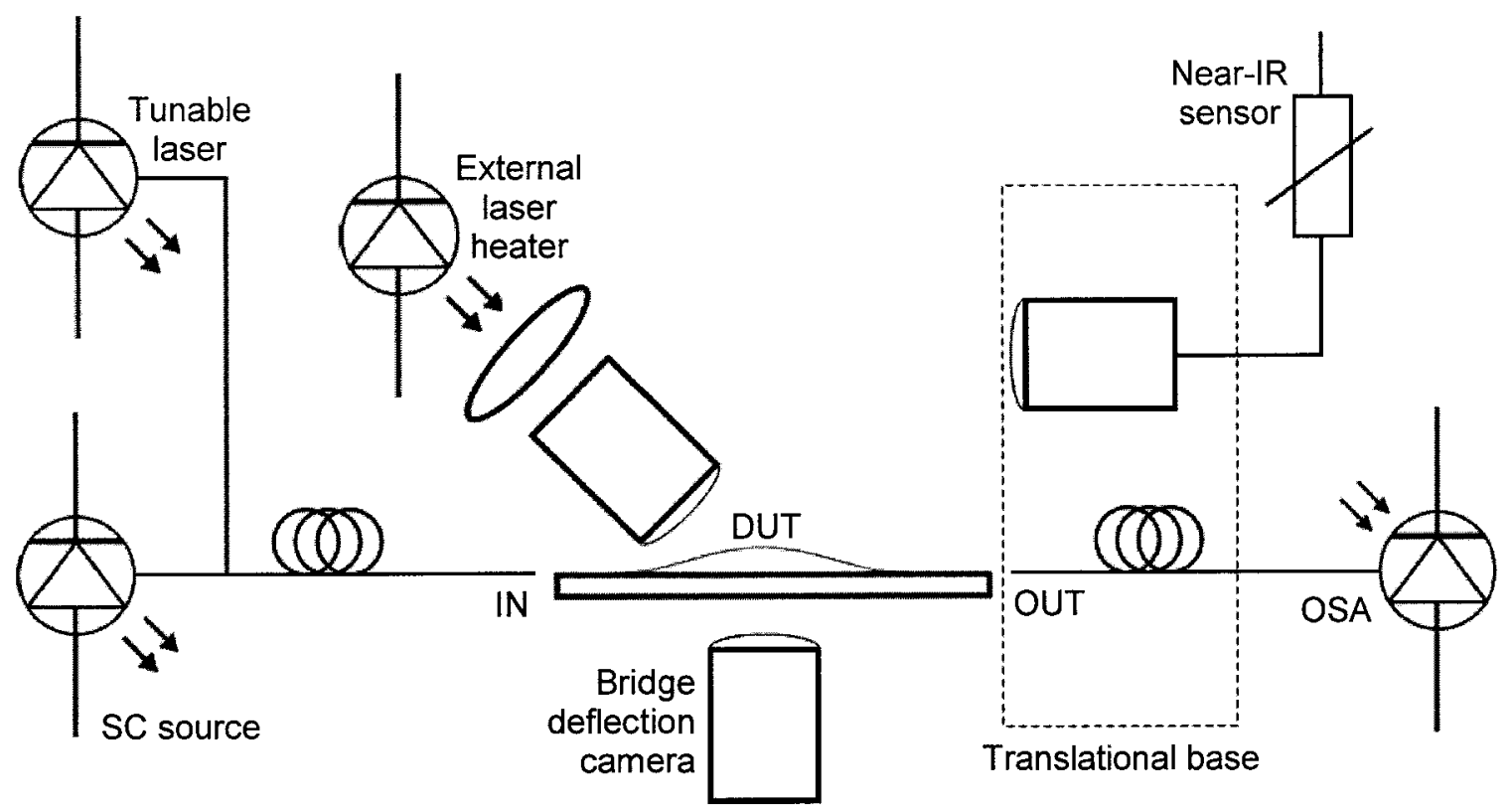

Figure 6.26. Schematic of thermomechano-optical test bench

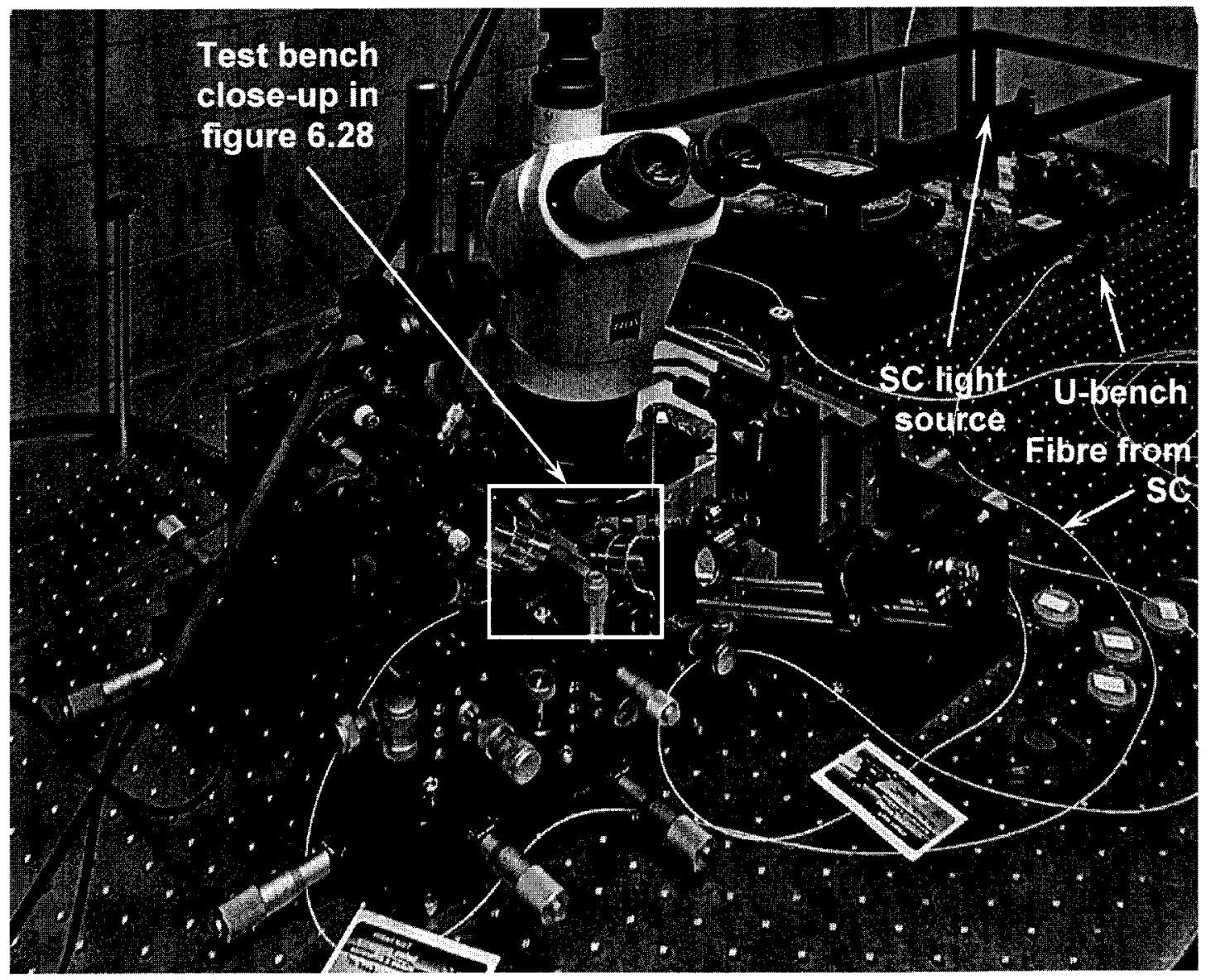

Figure 6.27. Thermomechano-optical test bench with SC light source (image reversed). 


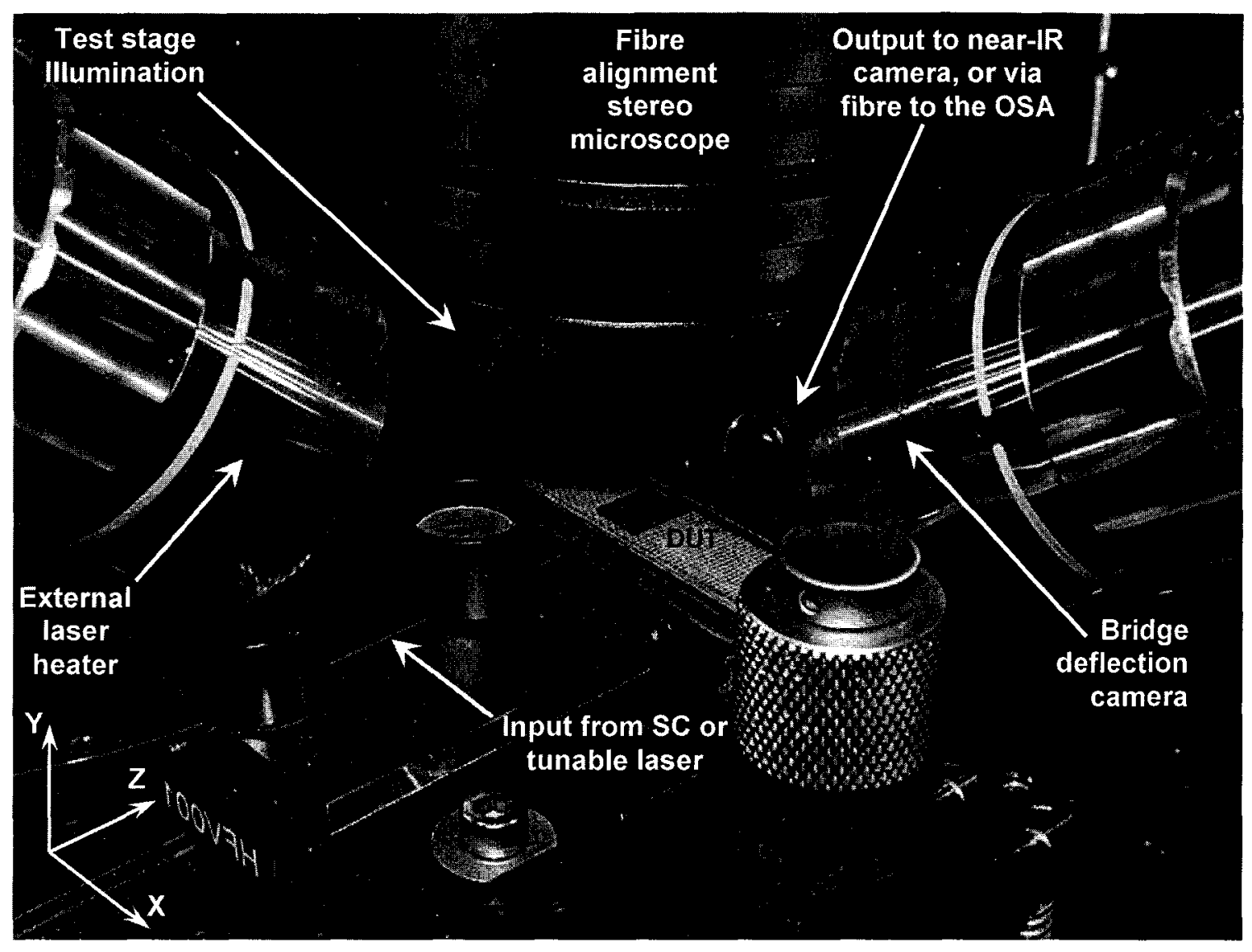

Figure 6.28. Core of thermomechano-optical test bench (image reversed).

The test stage that held the DUT had three translational axes of movement as well as rotation about the $\mathrm{X}$ and $\mathrm{Y}$ axis. This allowed a loose or packaged die to be oriented to the same $\mathrm{X}$ and $\mathrm{Y}$ axis of alignment as the input and output fibres. The alignment process would begin by lowering the DUT stage so either fibre could be positioned over a waveguide and observed through the fibre alignment microscope. The DUT was then rotated about its $\mathrm{Y}$ axis until the waveguide axis was parallel to the fibre. Next the attitude of the fibre above the DUT was observed through the bridge deflection camera. The DUT was rotated about the $\mathrm{X}$ axis until the DUT surface was parallel to the fibre as shown in figure 6.29. 


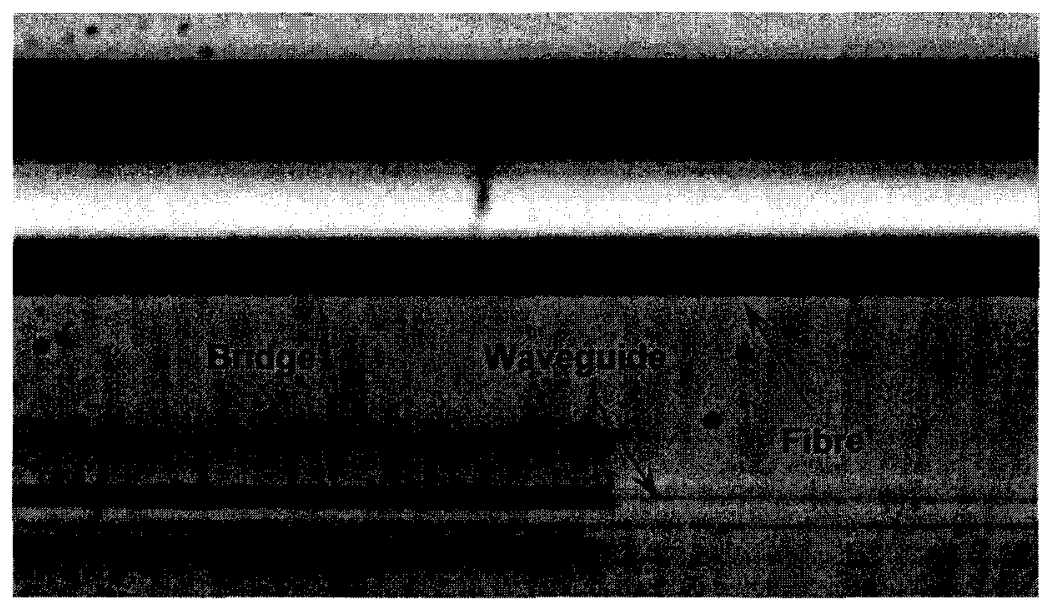

Figure 6.29. Part of the fibre to waveguide alignment process in three-space.

Figure 6.30 shows the result of a successful input fibre alignment to a straight waveguide. Illumination from light spreading along the slab portion of the waveguide at the input facet has coupled to adjacent waveguides. The output of the device waveguide has saturated the near-IR camera so its intensity cannot be compared to the intensity from the adjacent waveguides.

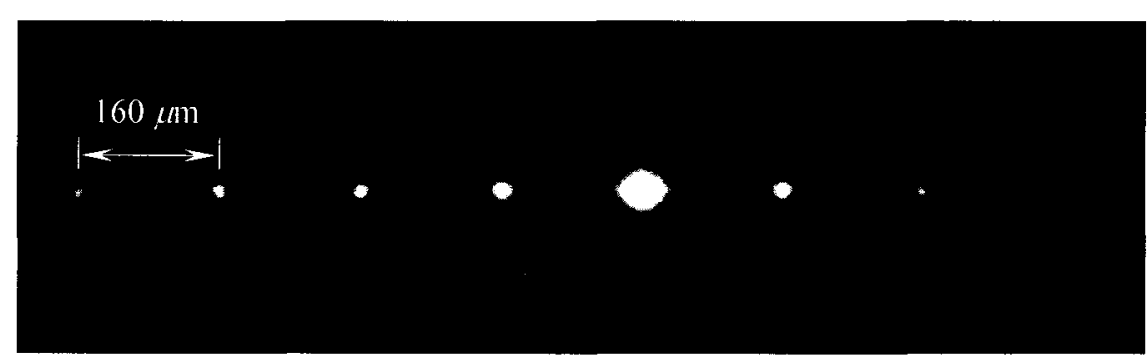

Figure 6.30. Near-IR image of $1 \mathrm{~mW} 1550 \mathrm{~nm}$ light from output facet.

Software to control the optical measurement process was initially developed by Scott Newman. The OSA was controlled by Agilent VEE Pro (ver. 6.01), a test and measurement program used to interface with test equipment via the general purpose interface bus (GPIB). The OSA was programmed to average 100 scans from a data capture run, taking 30 seconds to complete, for each waveguide filter temperature. Each scan contained 1200 wavelength measurements. Course measurements over a 
100-300 $\mathrm{nm}$ bandwidth were used to characterisation the test setup, while finer measurements over a 20 to $5 \mathrm{~nm}$ bandwidth were used to track the thermo-optical and thermomechano-optical shift of the Bragg dip.

The first baseline spectral response to be established was for the SC source itself. To accomplish this, the OSA was coupled directly to the output of the U-Bench with a Corning SMF-28 optical patch cable. Measurements were taken both with and without the $1100 \mathrm{~nm}$ edgepass filter. The filter caused a Fabry-Perot fringe period of $0.45 \mathrm{~nm}$ and an attenuation of $0.6 \mathrm{~dB}$, as shown in figure 6.31. This ripple would only be visible for the finer 20 and $5 \mathrm{~nm}$ bandwidth measurement sets.

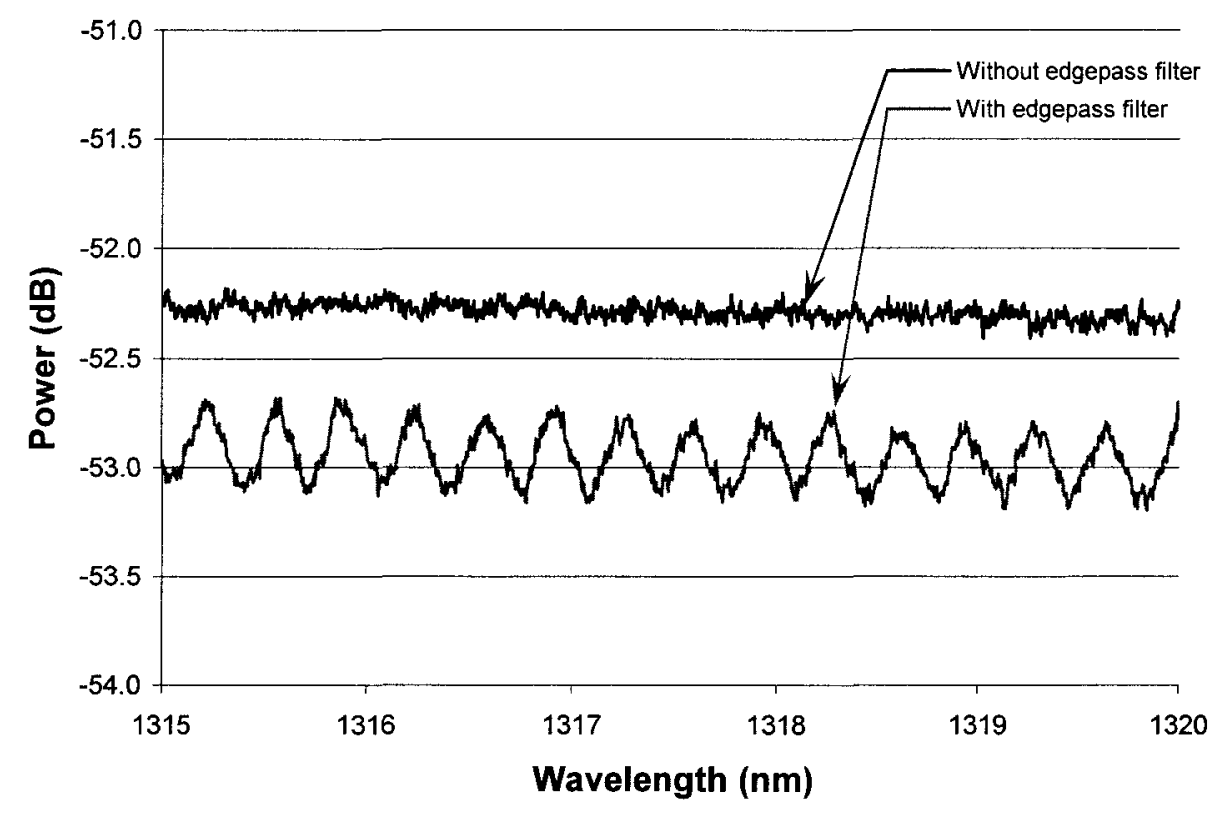

Figure 6.31. Direct spectral response of SC source to OSA via optical patch cord.

The experimental set up for base line characterisation of the fibre-to-fibre, and fibre-to-straight waveguide transmission, are shown in figure 6.32. This would standardise the system response against which the device performance measurements would be taken. 

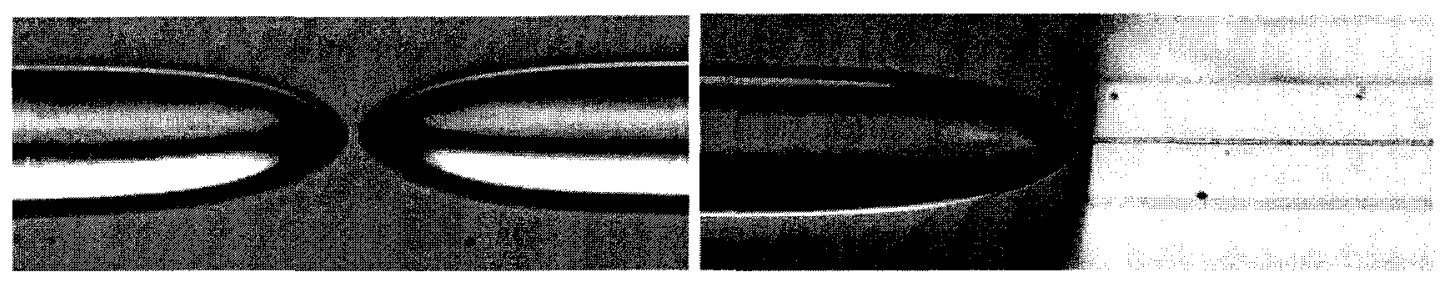

Figure 6.32. Fibre-to-fibre and fibre-to-straight waveguide alignment.

A course measurement set was taken for the transmission curve of the SC-to-OSA path over a $300 \mathrm{~nm}$ bandwidth of interest from 1300 to $1600 \mathrm{~nm}$. The lensed fibres were added to the optical path, and finally a straight rib waveguide from D2-16. The combination of all three transmission responses are plotted in Figure 6.33, showing the amount of loss from each element of the optical test setup.

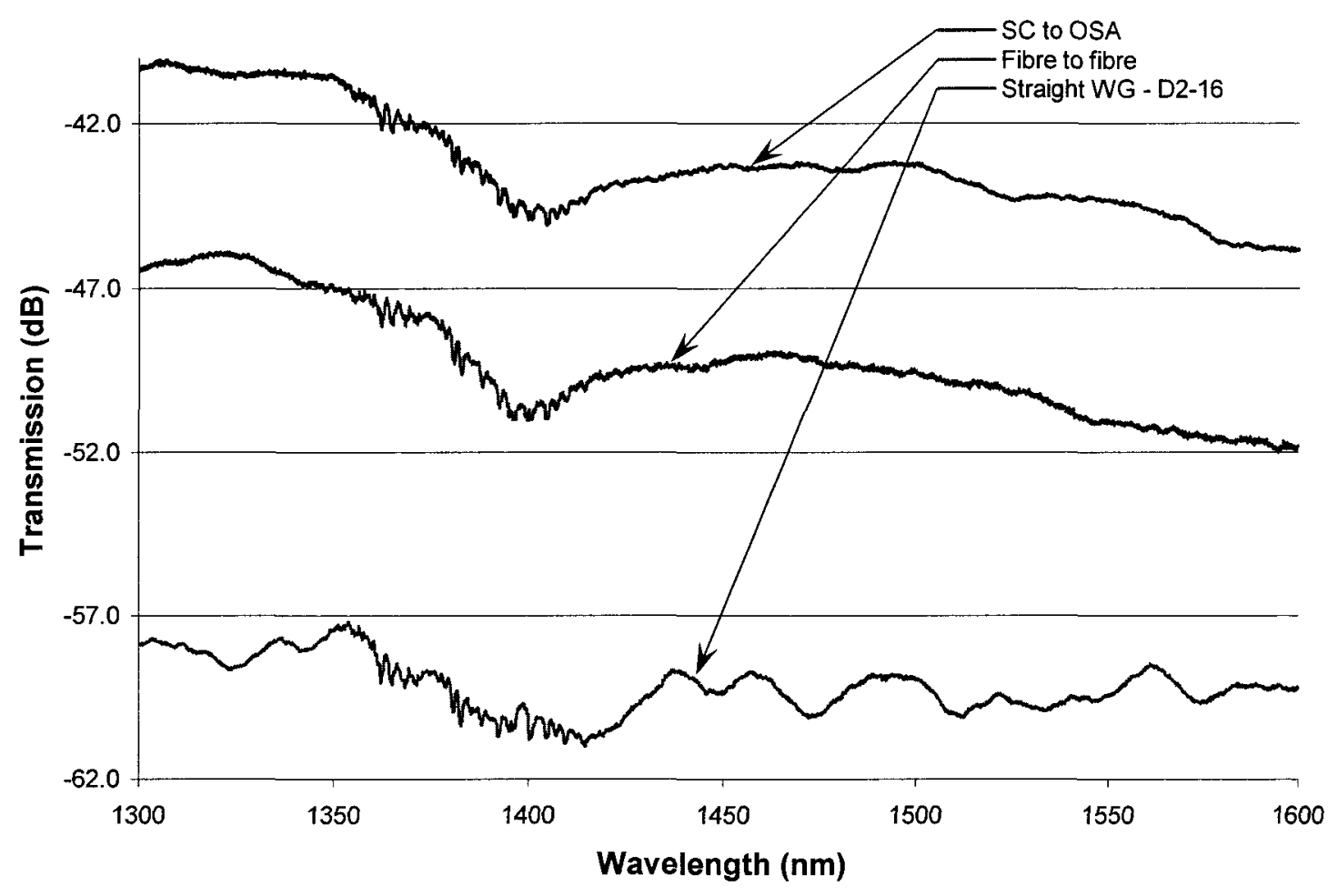

Figure 6.33. Transmission response to determine the amount of loss from each element of the test setup, taken over $1300-1600 \mathrm{~nm}$.

The spectral response of the SC alone shows a disturbance from $1360 \mathrm{~nm}$ to $1420 \mathrm{~nm}$. This indicates a region where measurements would not be reliable and dips 
could be mistaken for a Bragg dip. Adding the fibres into the optical path added a loss of $7.0 \mathrm{~dB}$. This was confirmed by replacing the SC optical signal with a $1550 \mathrm{~nm}$ signal at $1 \mathrm{~mW}$ of optical power from the tunable laser, and measuring $7.0 \mathrm{dBm}$ loss on the OSA. Inserting a straight waveguide from $\mathrm{D} 2-16$ into the $\mathrm{SC}$ optical path added another $8.3 \mathrm{~dB}$ of loss. The total SC-to-device-to-OSA loss at $1550 \mathrm{~nm}$ was $15.3 \mathrm{~dB}$. This compared to a total loss of $16.0 \mathrm{dBm}$ from a $1550 \mathrm{~nm}$ signal at $1 \mathrm{~mW}$ of optical power from the tunable laser.

The total loss for a waveguide with an integrated $1000 \mu \mathrm{m}$ Bragg grating filter was very close to that of an adjacent straight waveguide. Figure 6.34 plots the Bragg response alone, using D2-16 WG-1, and compares it to the response of a straight waveguide and the Bragg response together. For the purpose of comparison, the "Bragg Waveguide Alone" trace has been shifted $4 \mathrm{~dB}$ lower in the plot.

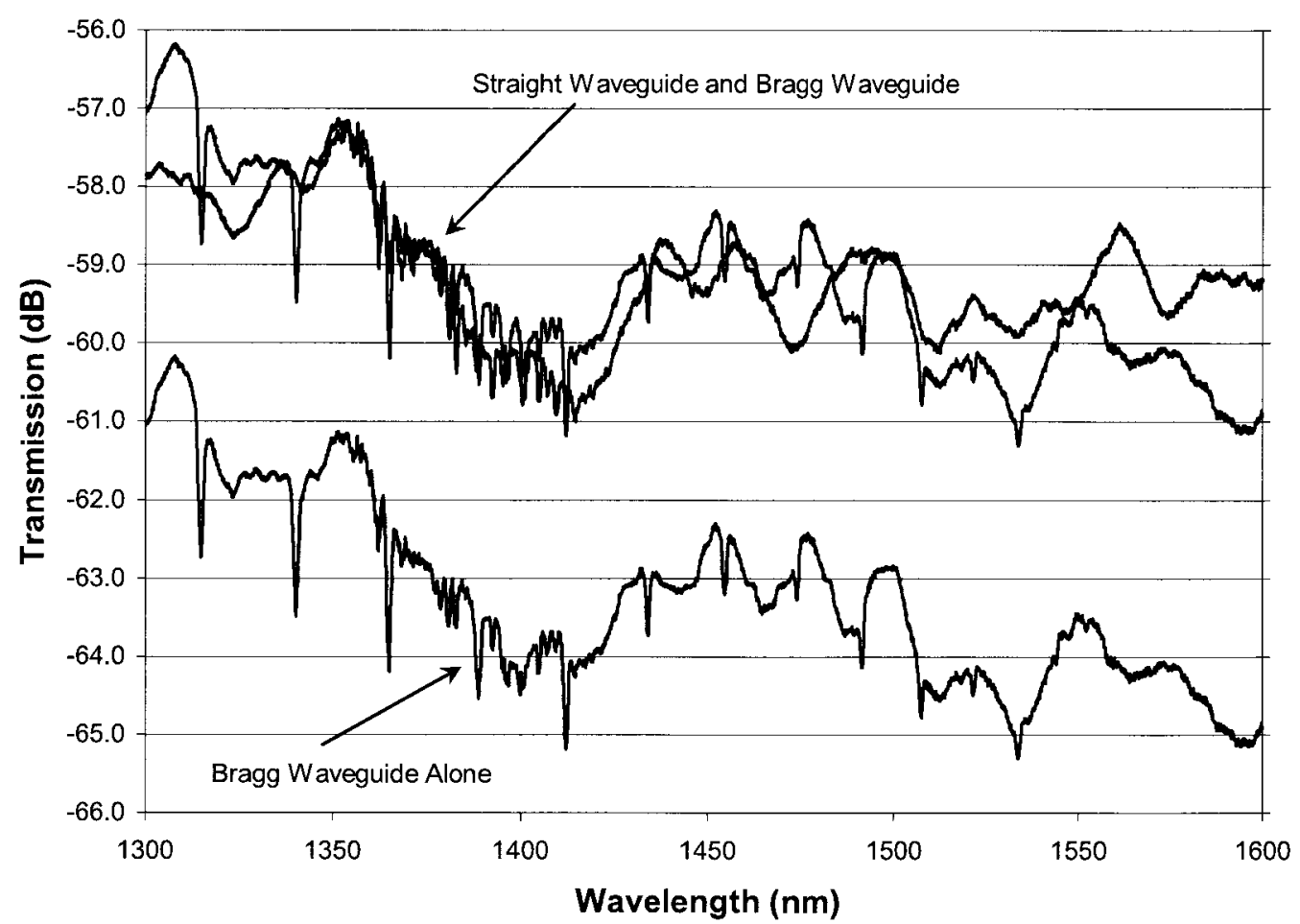

Figure 6.34. Comparison of the spectral response of a straight waveguide to a Bragg grating waveguide for D2-16. The Bragg wavelength response was shifted $4 \mathrm{~dB}$ down. 
Figure 6.35 shows a higher resolution spectral response for D2-16 WG-1 at a room temperature of $22{ }^{\circ} \mathrm{C}$. The fundamental mode of the Bragg wavelength, with a $\lambda_{B}$ of $1552.2 \mathrm{~nm}$, as well as four higher order leaky mode dips, are visible in the coarse scan. The coarse response is plotted over $60 \mathrm{~nm}$ on the left axis while a fine scan on the same experimental run is shown over $20 \mathrm{~nm}$ on the right axis. As the optical scan resolution becomes finer, the power measured by the OSA for a given wavelength decreases. The reason for this is that the power spectral density is finite across the spectrum that the $\mathrm{SC}$ provides. The smaller the bandwidth measured from the SC, the less power there is to measure. This figure also shows that a finer measurement revealed a sharper Bragg response due to the increased resolution. All optical characterisation was performed at the fine resolution.

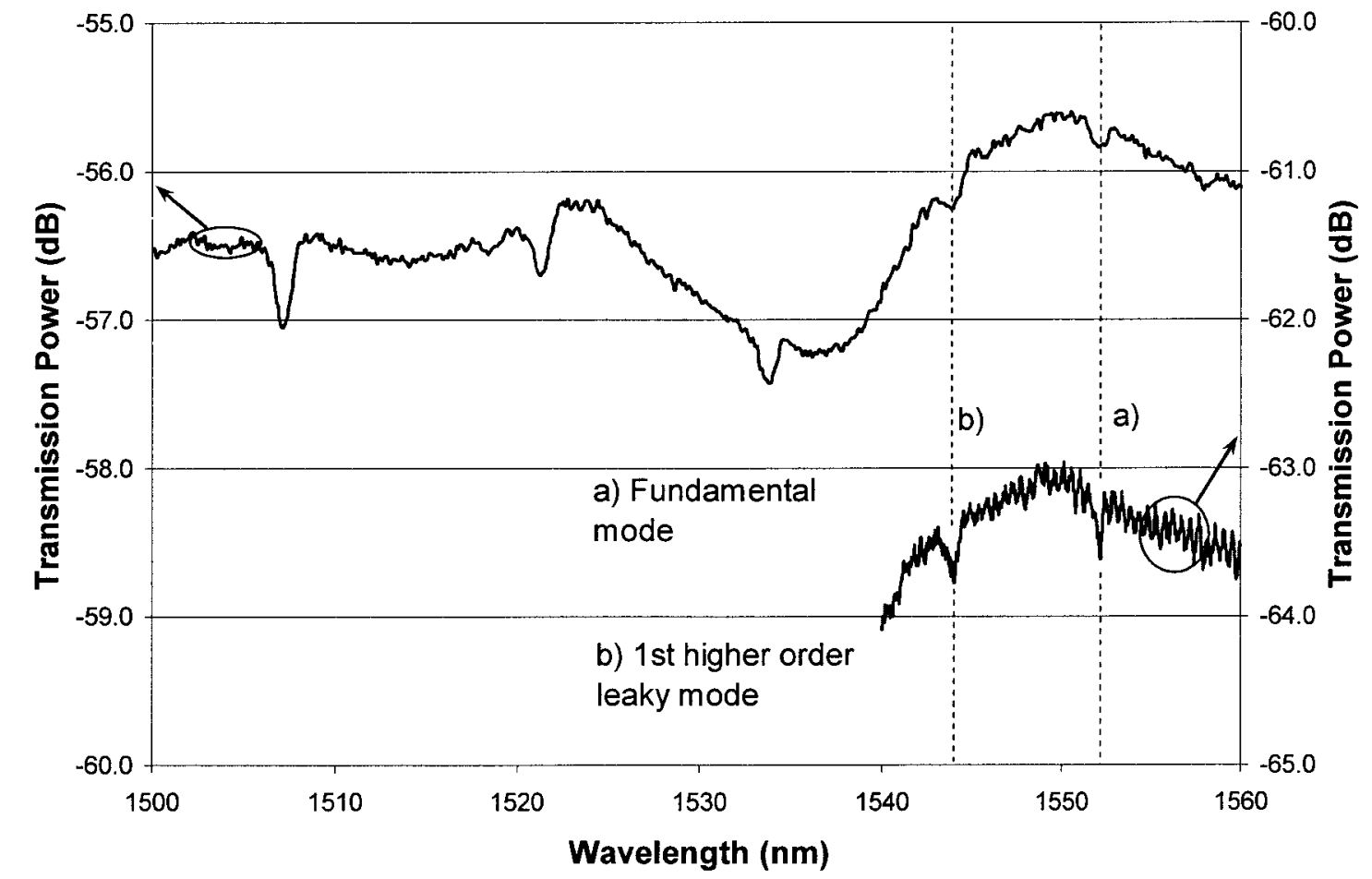

Figure 6.35. Transmission spectrum of a $1000 \mu \mathrm{m}$ Bragg grating at course and fine bandwidths for D2-16 WG-1 at room temperature. The fundamental mode Bragg wavelength is $1552.2 \mathrm{~nm}$. 
Figure 6.36 focuses on a higher order Bragg dip, at room temperature, of a $1000 \mu \mathrm{m}$ Bragg grating filter waveguide above WG-1 (located on the mask in figure 4.14). In an ideal grating, where the mode of light is incident on only one surface for each period of the Bragg grating, the Bragg dip would come to a single point. The Bragg grating mask used in the etch process was $8 \mu \mathrm{m}$ wide. This width allowed space to ensure the $5 \mu \mathrm{m}$ wide rib waveguide mask could be aligned within the grating. The duration of the grating etch would determine its depth and duty cycle. The grating duty cycle is defined as the ratio between the axial width of a single grating segment to the period of the grating, as defined in figure 3.6. The rib waveguide mask would protect the grating over its width so that the grating depth and duty cycle would not change during the rib etch. The portions of the grating not protected by the rib waveguide mask would continue to etch, however, increasing the grating duty cycle in this region. This can be seen in figure 6.37 where the duty cycle of the Bragg grating filter is 0.29 , while the duty cycle of the grating on either side is 0.61 .

In a numerical survey on Bragg reflectors on SOI waveguides, I. Giuntoni et al. [116], demonstrated that a change in grating duty cycle for a fixed Bragg grating pitch would cause a shift in the Bragg wavelength. The effect of two duty cycles with the same Bragg grating pitch likely caused more than one peak within the Bragg dip wavelength. This phenomena was also observed in the Bragg dips of the PC devices that were characterised at IMS-NRC. To locate the wavelength of the Bragg dip for measurement purposes, the outer sides of the complete Bragg dip were extended to a point that would indicate the location of the Bragg wavelength. This process was used to locate the centre of all Bragg dips, whether they were easily defined or not, to maintain consistency in the 
measurements.

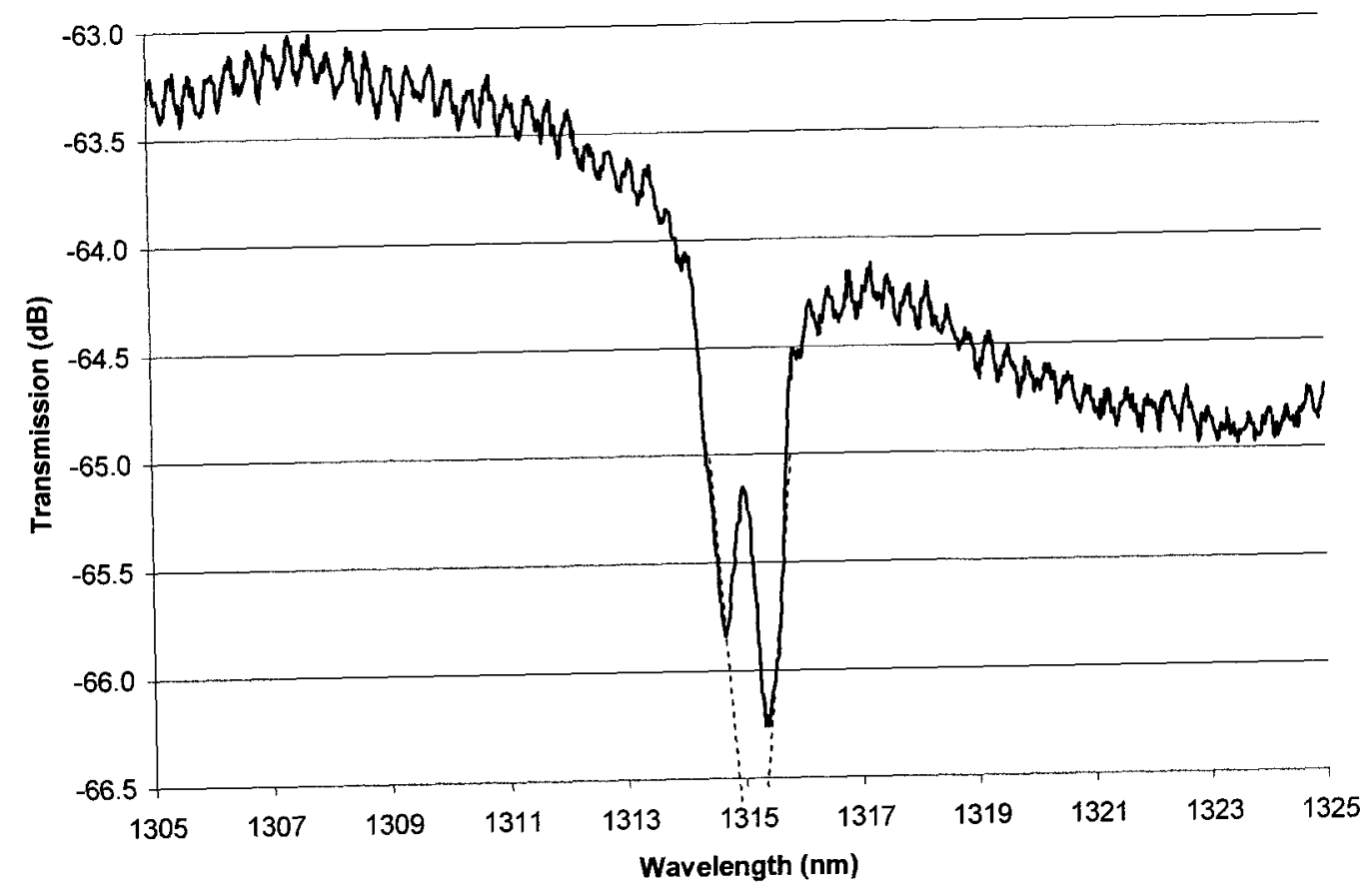

Figure 6.36. Plot of a higher order mode Bragg dip from D2-16 showing two peaks and the method to resolve the Bragg wavelength, $1315.1 \mathrm{~nm}$ in this case.
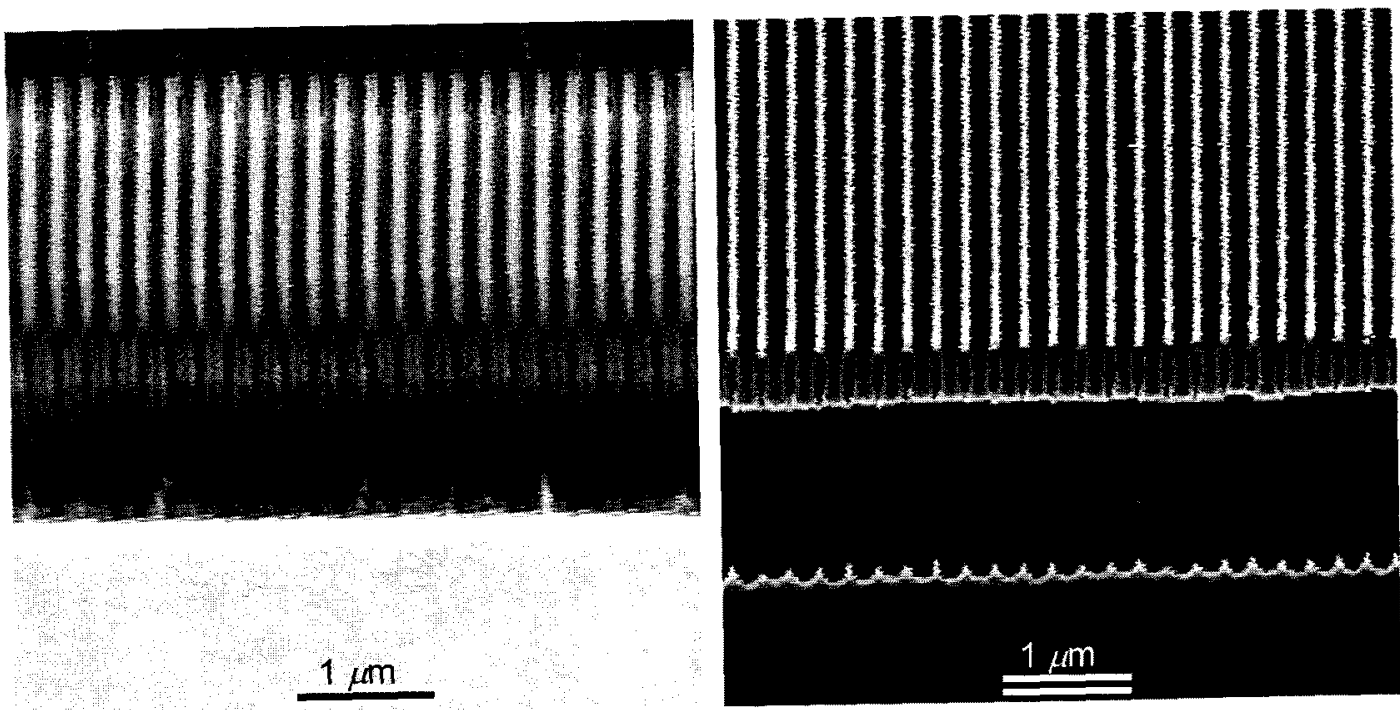

Figure 6.37. SEM images of D2-24 and D2-14 showing the rib waveguide / Bragg grating overlap that causes two different duty cycles for the same Bragg grating pitch. 


\subsubsection{Thermo-optical characterisation}

Thermo-optical characterisation was completed on devices with both integrated heaters (D2-22) and those externally heated (such as D2-16). The schematic for the integrated heater thermo-optical test setup is shown in figure 6.38. This characterisation procedure made use of the same test setup that thermo-mechanical and thermomechanooptical experimentation did, as shown by the schematic in figure 6.26 .

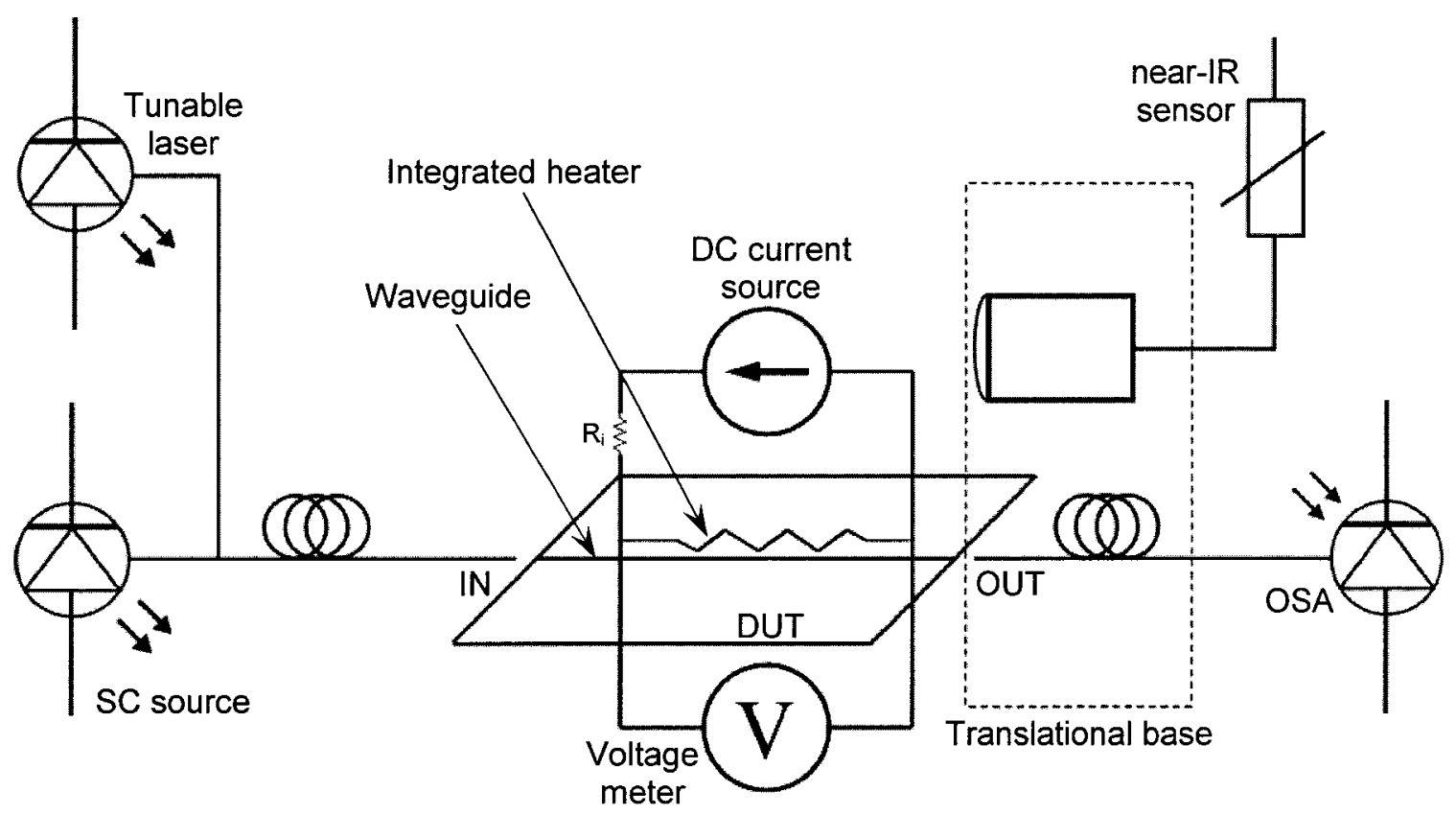

Figure 6.38. Schematic for thermo-optical characterisation of device with integrated heaters.

A planar view of the packaged die, D2-22, with cleaved waveguide facets and wirebonded integrated heaters, is shown in figure 6.39. The experimental setup allowed simultaneous electro-thermal and thermo-optical characterisation to take place. The electro-thermal response was calculated using the process outlined in section 6.1.2 and the thermal shift in the Bragg wavelength was measured using the optical characterisation process outlined in section 6.3.1. A one minute pause was included between each setting to allow the system to thermally stabilise before each electrical and optical measurement. 


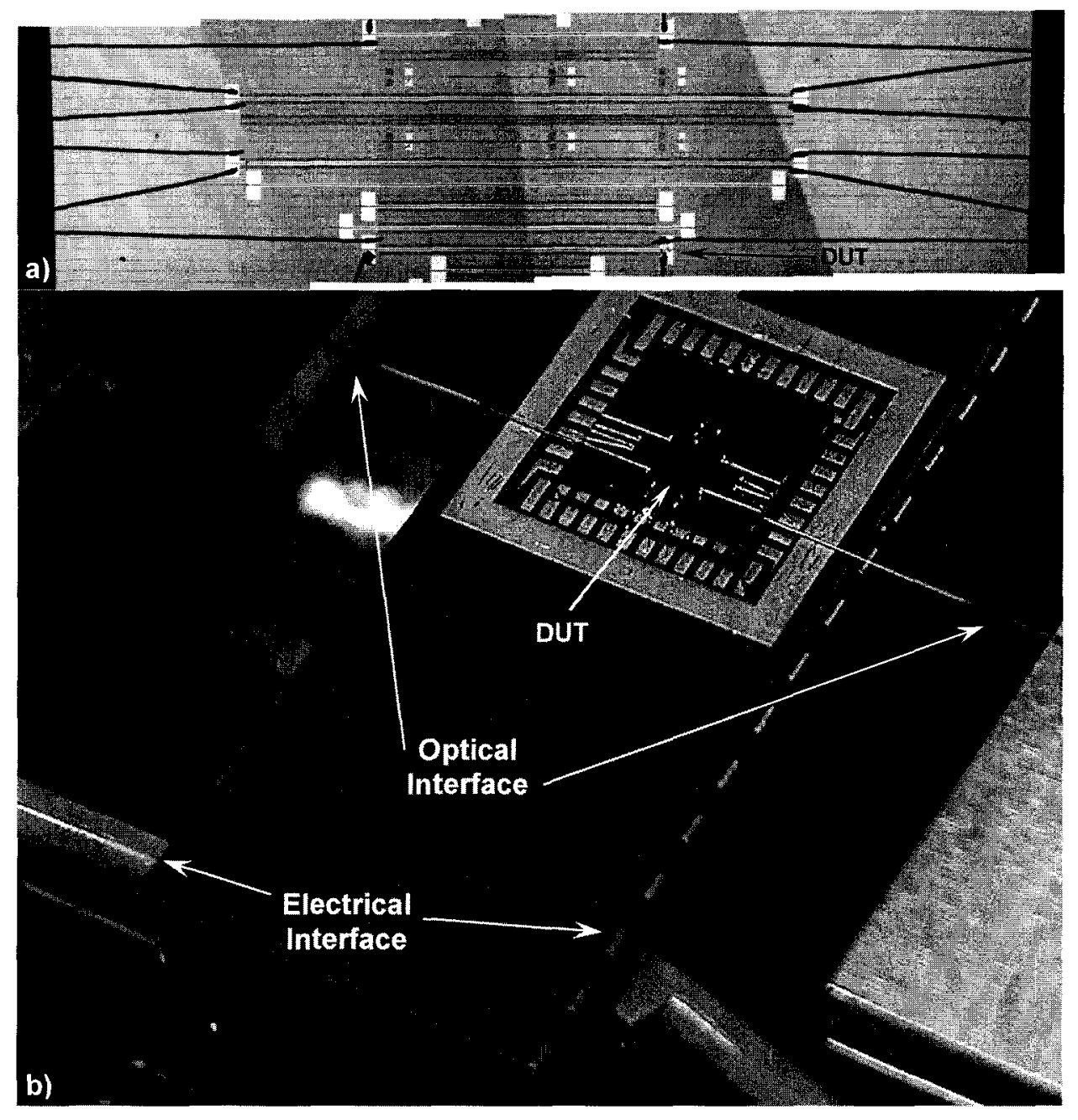

Figure 6.39. Packaged D2-22 device undergoing simultaneous electro-thermal and thermo-optical characterisation.

The thermal shift of the fundamental mode Bragg wavelength, using integrated heaters, was experimentally determined to be $82 \mathrm{pm} /{ }^{\circ} \mathrm{C}$. The theoretical value of $83 \mathrm{pm} /{ }^{\circ} \mathrm{C}$ was calculated in chapter 4 . To compare the theoretical rate with the experimental rate, $n_{\text {eff }}$ was chosen to match the value of $\lambda_{B}$ measured at room temperature. This would not change the theoretical rate, but it would allow the two slopes to begin at the same point. The comparison is plotted in figure 6.40. The uncertainty of the experimental slope, within a $95 \%$ confidence level, was $\pm 5 \mathrm{pm} /{ }^{\circ} \mathrm{C}$.

I. Giuntoni et al. [117] characterised a similar grating rib waveguide on SOI, in 
which the entire die was thermally tuned via a Peltier heater. They measured an experimental rate of $77 \mathrm{pm} /{ }^{\circ} \mathrm{C}$. S. Homampour et al. [118] also used bulk heating of an SOI die, containing Bragg gratings in rib waveguides, to measure an experimental value of $80 \mathrm{pm} /{ }^{\circ} \mathrm{C}$.

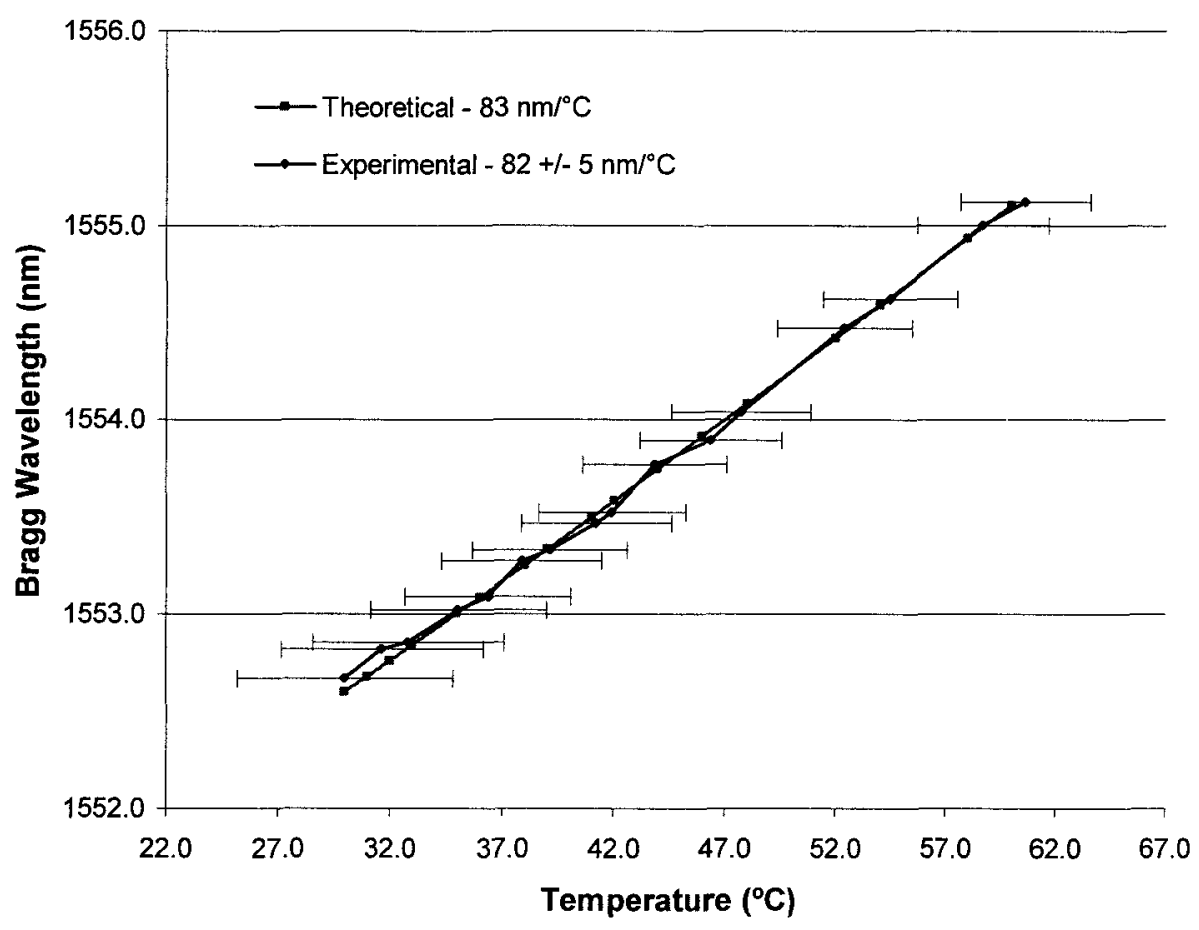

Figure 6.40. The shift in Bragg wavelength versus the temperature from an integrated heater of a Bragg grating filter within a waveguide.

Although the thermal sensitivity of the Bragg wavelength in $\mathrm{pm} /{ }^{\circ} \mathrm{C}$ for all three devices are in agreement, there is an important distinction to make between the other two devices and the device described in this thesis. The two cited examples made use of a tunable thermal source to heat the entire die. Through this method of characterisation the investigators were able to measure the temperature change directly from the heater as it thermally shifted the Bragg wavelength. The temperature change from the integrated heaters described in this thesis was calculated indirectly. This is an important distinction 
to make since the indirect method of thermal measurement will also be used to translate the drive current used by the LD heater into an actual device temperature.

During the thermomechano-optical experiments it was discovered that the strength of the fundamental Bragg dip became too weak to measure in the post-buckle regime. The Bragg dip from the higher order leaky modes remained strong enough to measure through out the entire range of operation. In order to complete characterisation across the entire pre- to post-buckle region of operation it was necessary to measure the thermal shift of the Bragg wavelength at one of the higher order leaky modes.

Work by C. Chen and J. Albert show that the differential wavelength shift of higher order modes relative to the fundamental mode is constant [119]. The higher order Bragg dips will thermally shift at the same rate as the fundamental Bragg dip. Optical measurements of these higher order Bragg dips will be used to map the integrated heater thermal tuning rate from D2-22 to the LD current thermal tuning rate from D2-16.

The thermo-optical response was measured on D2-22 for higher order leaky mode Bragg dips starting with a room temperature wavelength of $1318.2 \mathrm{~nm}$ and $1456.1 \mathrm{~nm}$. To confirm that the thermal response of the filter at these higher order modes could be considered equivalently to the fundamental Bragg dip, the same thermo-optic characterisation was performed. The thermal sensitivity for two higher order Bragg dips was plotted for the $1300 \mathrm{~nm}$ and $1400 \mathrm{~nm}$ regions as shown in figure 6.41. The rates measured were $76 \mathrm{pm} /{ }^{\circ} \mathrm{C}$ and $77 \mathrm{pm} /{ }^{\circ} \mathrm{C}$ respectively. With these rates it was now possible to map the integrated heater thermal sensitivity of the Bragg wavelength for D2-22, to the LD current sensitivity for the Bragg wavelength of D2-16 for a similar higher order Bragg dip. By heating the waveguide filter in the pre-buckle regime, a rate 
for the Bragg shift to LD current change could be measured and mapped to the rate measured for the integrated heaters in the packaged device.

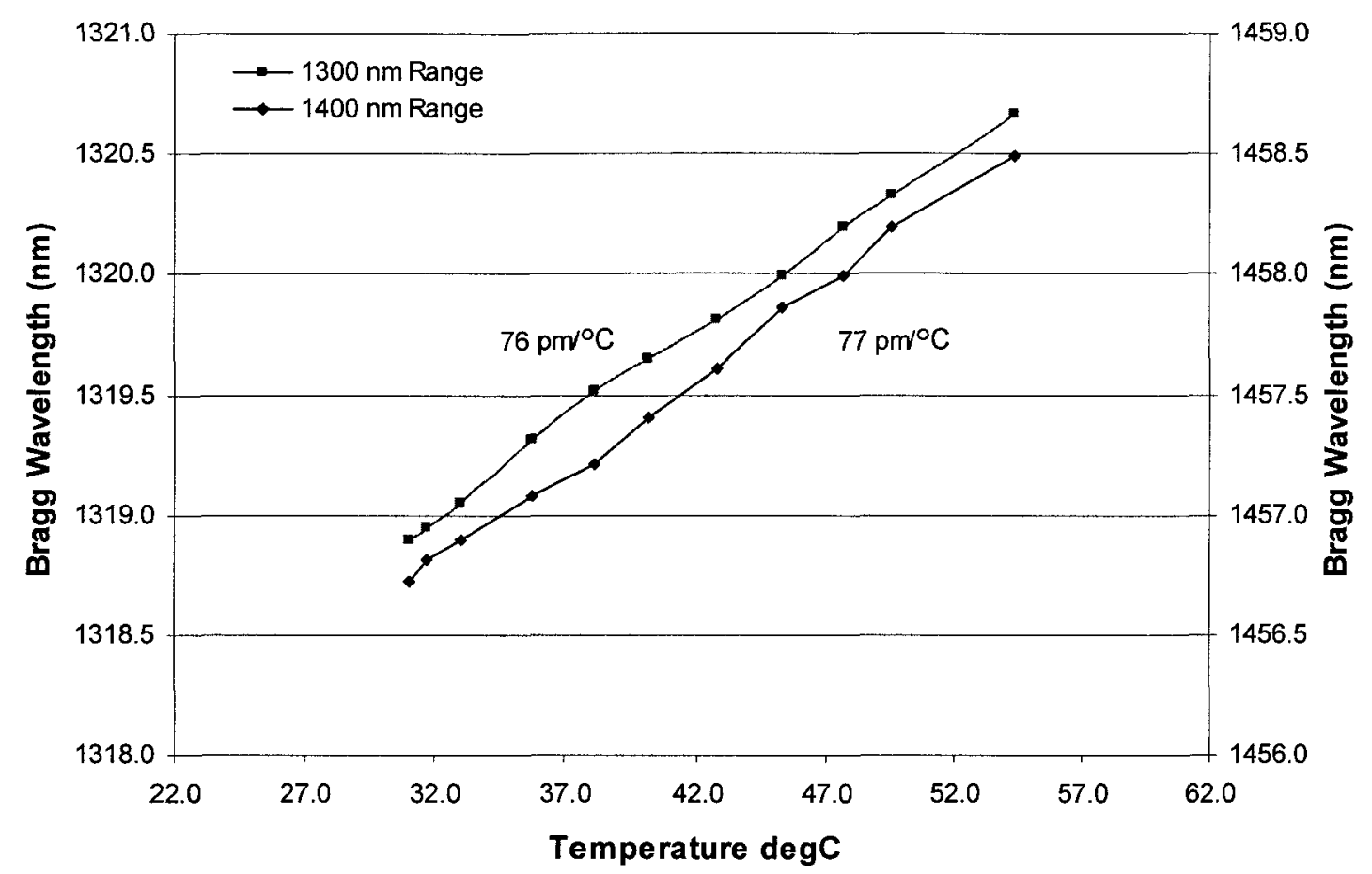

Figure 6.41. Comparison of thermal sensitivity in the Bragg wavelength of higher order leaky modes on D2-22.

The shift of the higher order Bragg peak in the same $1300 \mathrm{~nm}$ region of the D2-16 WG-1 device was measured in $1.0 \mathrm{~mA} \mathrm{LD}$ increments, for both increasing and decreasing current in the pre-buckle regime, and plotted in figure 6.42. The sensitivity of the Bragg wavelength to the change in LD current was $71 \mathrm{pm} / \mathrm{mA}$ for both increasing and decreasing LD current. 


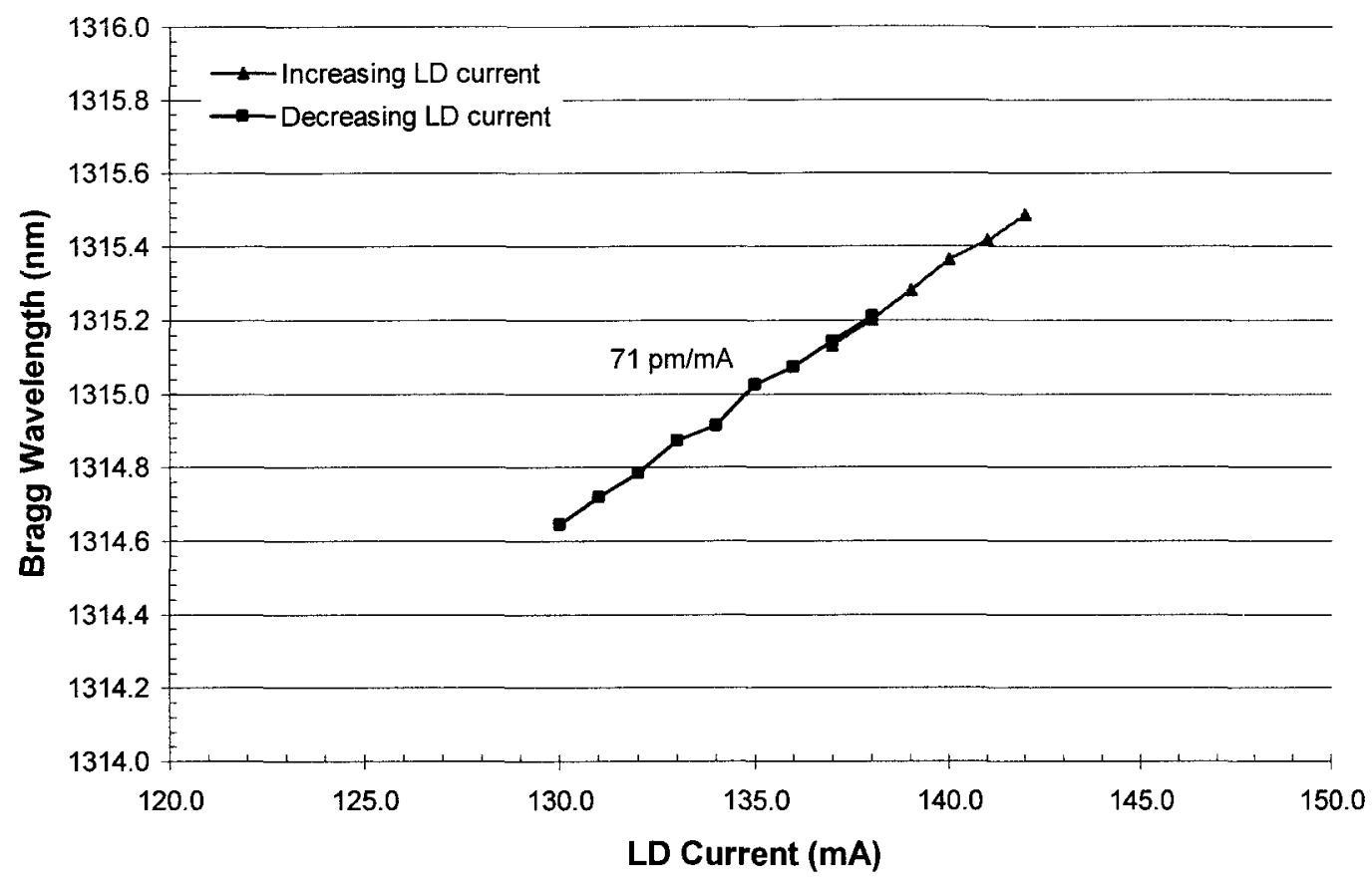

Figure 6.42. Shift in Bragg wavelength of higher order leaky mode versus laser diode current using D2-16 in the pre-buckle regime.

The $71 \mathrm{pm} / \mathrm{mA}$ Bragg wavelength shift from the LD external heater drive current was compared to the $76 \mathrm{pm} /{ }^{\circ} \mathrm{C}$ Bragg wavelength shift from the integrated heaters for the same higher order Bragg dip. Dividing the LD thermal sensitivity by the integrated heater thermal sensitivity:

$$
\frac{71 \frac{p m}{m A}}{76 \frac{p m}{{ }^{\circ} C}}
$$

results in a conversion factor of $0.934^{\circ} \mathrm{C} / \mathrm{mA}$. By using the common reference point of room temperature, it is possible to correlate the thermo-optical response from the integrated heater to the thermo-optical response of the LD external heater. Room temperature measured to be $22.0^{\circ} \mathrm{C}$ during experimentation. The effective $\mathrm{LD}$ current at room temperature was $98 \mathrm{~mA}$, as determined in figure 6.10. The current axis for external 
heating from $L D$ current (figure 6.42) can be converted to a temperature axis for an integrated heater (figure 6.41) using the following relationship :

$$
\text { temperature }=22^{\circ} \mathrm{C}+(\text { LD current }-98 \mathrm{~mA}) 0.934^{\circ} \mathrm{C} / \mathrm{mA}
$$

When this conversion is made to the thermo-mechanical waveguide buckle characterisation that was plotted in figure 6.21 , the buckle height behaviour of the waveguide bridge could now be plotted against temperature. The conversion process resulted in a critical buckling temperature between $73.4{ }^{\circ} \mathrm{C}$ and $73.8^{\circ} \mathrm{C}$. The critical buckling temperature calculated in chapter 4 was $69.0{ }^{\circ} \mathrm{C}$. This $\mathrm{LD}$ current to temperature translation was possible because: 1) it was shown that the thermo-optic shift of the Bragg dip for the integrated heaters was linear, and the thermo-optic shift for the external LD heater of the same Bragg dip was linear over the pre-buckle region; and 2) both the room temperature value of the integrated heater and the effective LD drive current at room temperature was known, giving the two slopes a common starting point.

To summarise, the thermal response for the Bragg wavelength shift provided by a LD heated device was determined indirectly by measuring the thermo-optical effect in a device with integrated heaters and then mapping that rate to the pre-buckle thermooptical effect in a device with an external heater. Using this mapping calculation, the plot of buckle height versus LD current, shown in figure 6.21 , can be replotted with temperature along the $\mathrm{x}$-axis. The bridge deflection height could now be plotted as a function of temperature, as shown in figure 6.43. Since it was demonstrated that the LD current to power response is linear over the entire range of operation, this mapping can be carried into the post-buckle range of operation as well.

The critical buckle height for a $4.1 \mu \mathrm{m}$ thick, $2000 \mu \mathrm{m}$ long "T" cross-section 
bridge measured $15.1 \mu \mathrm{m}$, and compared well to the theoretical value of $14.8 \mu \mathrm{m}$. The temperature that the critical buckling mode occurred at was experimentally determined to be between $73.4{ }^{\circ} \mathrm{C}$ and $73.8{ }^{\circ} \mathrm{C}$, which is close to the theoretical value of $69.0{ }^{\circ} \mathrm{C}$ considering the uncertainty of $\pm 4.8{ }^{\circ} \mathrm{C}$ to $\pm 3.0^{\circ} \mathrm{C}$ that was calculated in section 6.1.2. The experimental work described thus far has outlined a procedure for the final device characterisation; that of a thermomechanically tunable Bragg grating filter on a suspended rib waveguide.

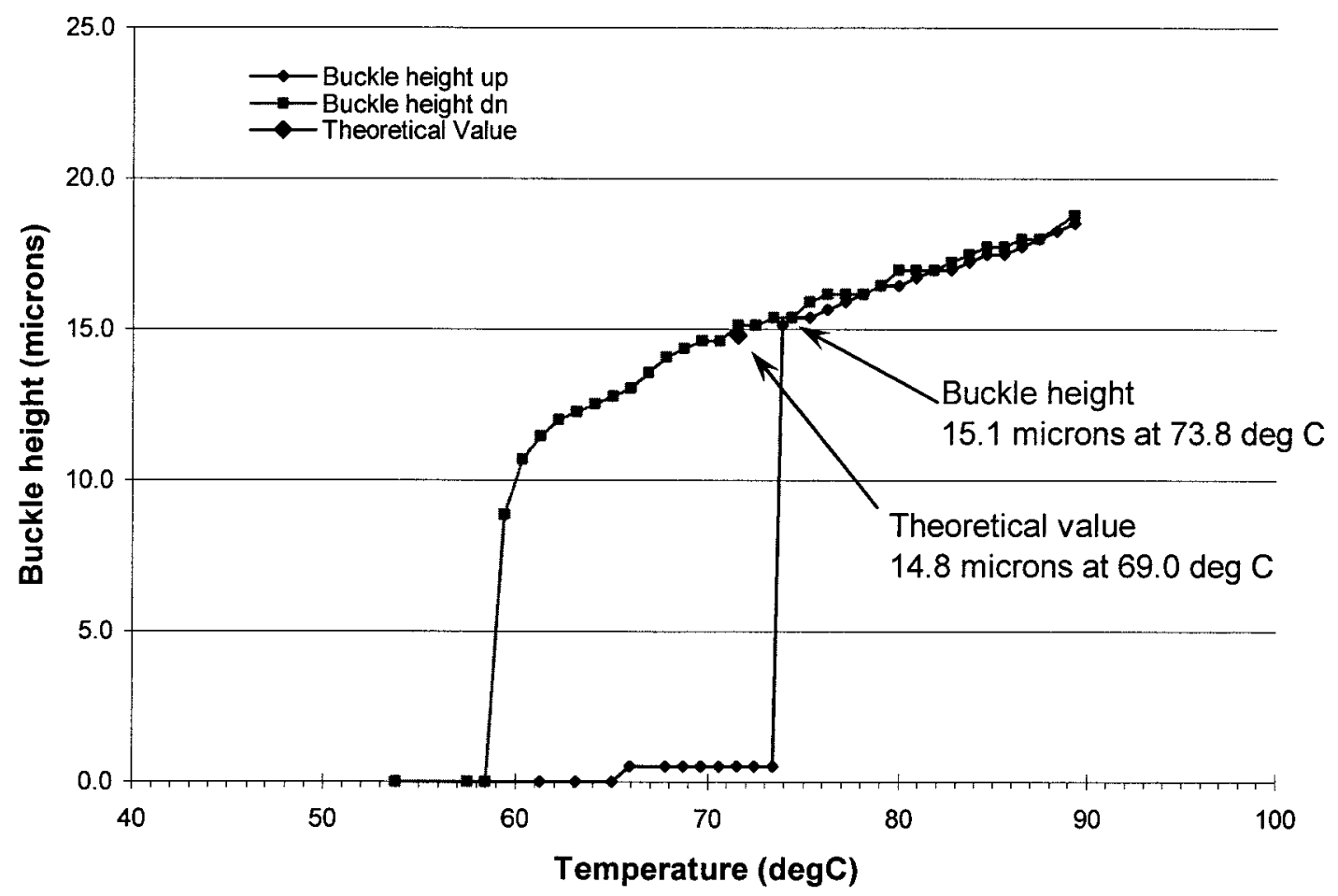

Figure 6.43. Waveguide bridge deflection versus the waveguide bridge temperature over the pre- to post-buckle regime for D2-16 WG-1.

\subsubsection{Thermomechano-optical characterisation}

The experimental setup for external thermal tuning of a Bragg filter on a rib waveguide bridge is shown in figure 6.44 and corresponds to the schematic outlined in figure 6.26. The bridge deflection camera was used in this experimental work to ensure 
the laser light was aligned to the same position on the waveguide bridge that it had been for the thermo-mechanical analysis.

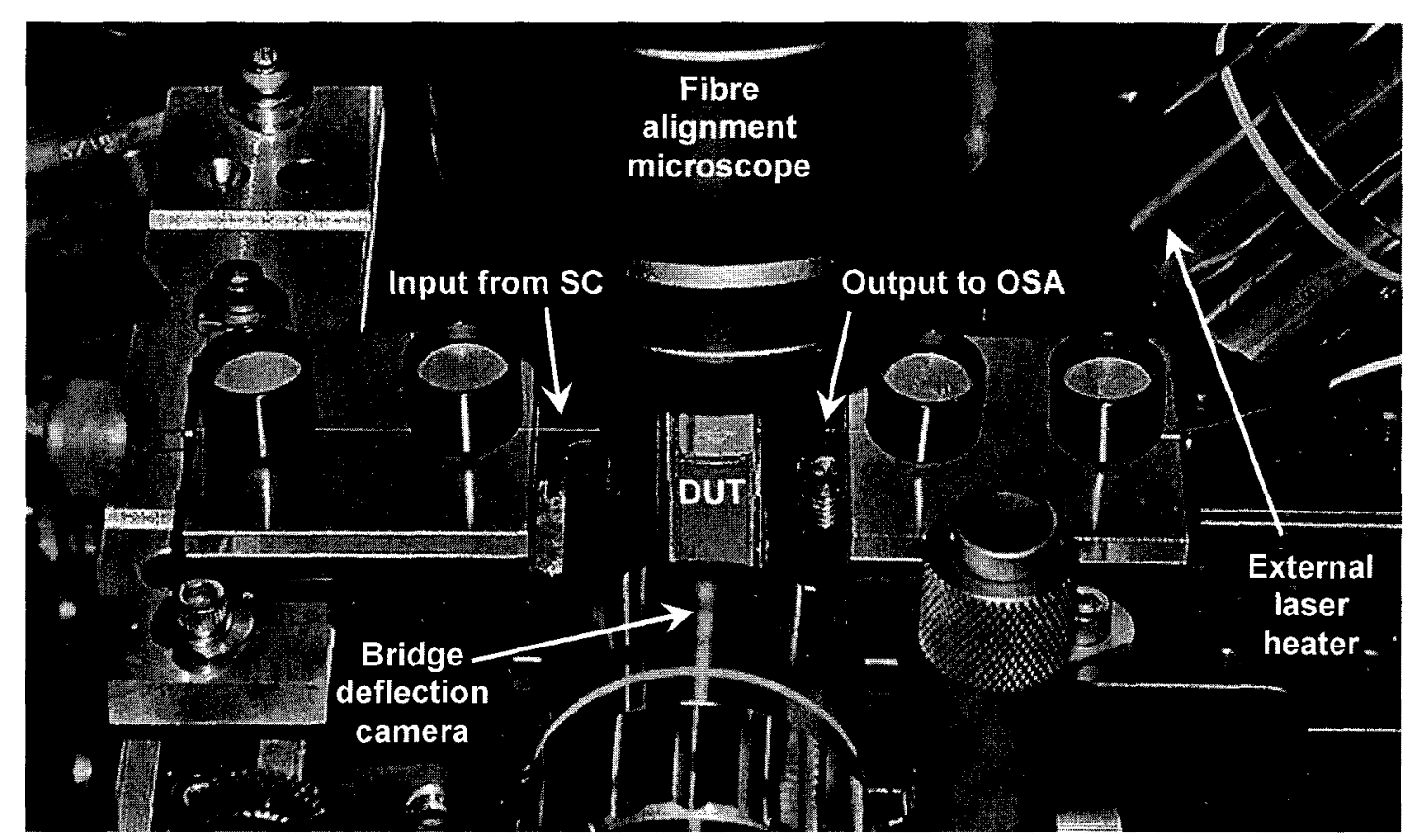

Figure 6.44. Unpackaged D2-16 device undergoing thermomechano-optical characterisation.

The thermo-optical characterisation procedure described in section 6.3 .2 was used in conjunction with the thermo-mechanical characterisation procedure described in section 6.2 to complete the thermomechano-optical characterisation of D2-16 WG-1. Over the process of developing the experimental procedure it became apparent that when the bridge buckled, the fundamental Bragg wavelength became too weak to measure. Therefore a stronger higher order mode Bragg dip was used to fully characterise the device performance.

The spectral response for each temperature was captured over $20 \mathrm{~nm}$ and $5 \mathrm{~nm}$ bandwidth resolution. The $20 \mathrm{~nm}$ resolution provided an orientation for the location of the Bragg dip, while the $5 \mathrm{~nm}$ resolution was used for measurement of the centre of the 
Bragg dip. The full scale for $20 \mathrm{~nm}$ resolution is $2.0 \mathrm{~dB}$, while the full scale for $5 \mathrm{~nm}$ resolution is $1.5 \mathrm{~dB}$. To help track the shift of the Bragg dip over the entire range of operation, a screen capture of the plot for each 1.0 mA LD current increment was taken. The resulting images were stepped through manually on screen to ensure the dip location was being followed properly and to keep track of the outer sides of the complete Bragg dip so that adjacent peaks would not be mistakenly included in the measurement.

Figures 126 through 131 show the Bragg dips of primary interest as well as the method of locating the centre of the Bragg dips graphically. Locating the centre of the Bragg dip for the pre-bump configuration at $145.0 \mathrm{~mA}$, the post-bump configuration at $146.0 \mathrm{~mA}$, and the pre-buckle configuration at $153.0 \mathrm{~mA}$ were accomplished by superimposing lines on the outer sides of the complete Bragg dip. The intersection of these lines were used to locate where the centre of the Bragg dip would be. This technique was performed on all Bragg dips, regardless of strength, to ensure consistency of measurement.

This technique was effective in tracking the shift of the Bragg dip when combined with the incremental $1.0 \mathrm{~mA} \mathrm{LD}$ current. In the post-buckle regime, the centre between double peak was used to measure the shift of the Bragg peak. The FWHM bandwidth, $\delta_{3 d B}$, for the pre-bump, post-bump and pre-buckle Bragg dips was $1.1 \mathrm{~nm}, 0.77 \mathrm{~nm}$, and $0.78 \mathrm{~nm}$ respectively. The strength of the dip was $1.0 \mathrm{~dB}$. The $\delta_{3 d B}$ for the post-buckle Bragg dip was $3.7 \mathrm{~nm}$ with a strength of $0.5 \mathrm{~dB}$. 


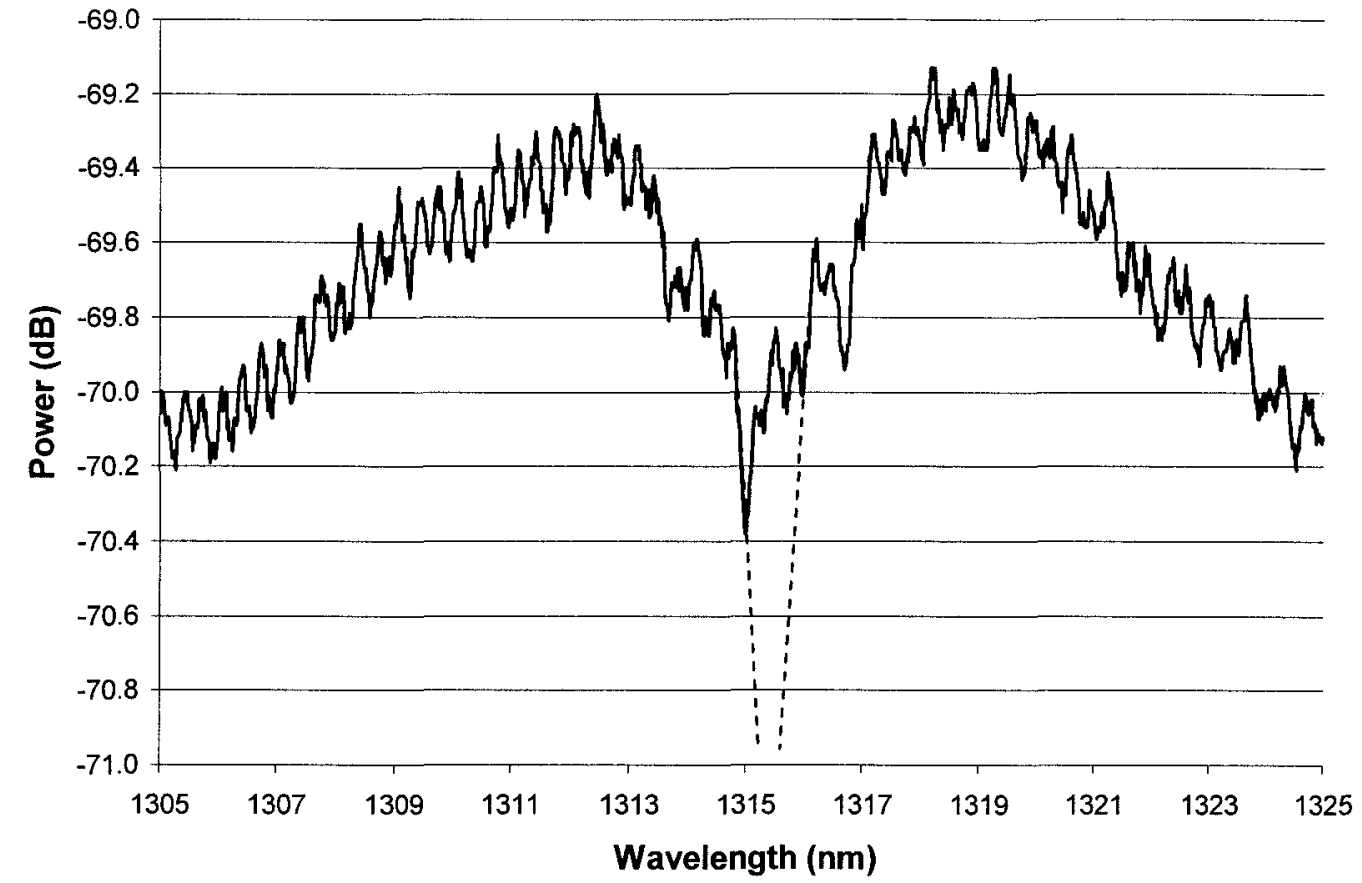

Figure 6.45. $20 \mathrm{~nm}$ resolution scan of pre-bump Bragg wavelength plot at $145.0 \mathrm{~mA}$.

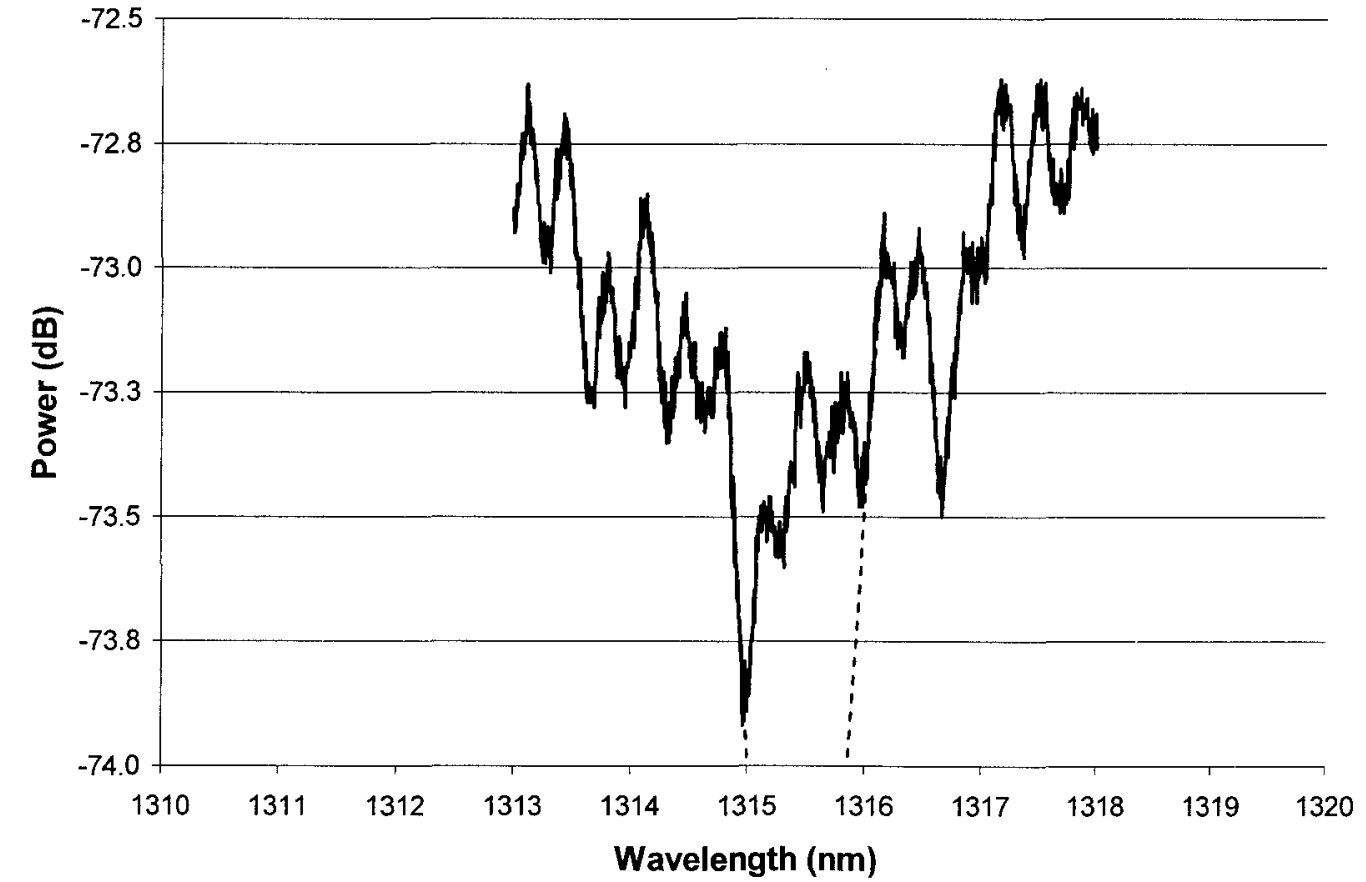

Figure 6.46. $5 \mathrm{~nm}$ resolution scan of pre-bump Bragg wavelength plot at $145.0 \mathrm{~mA}$. 


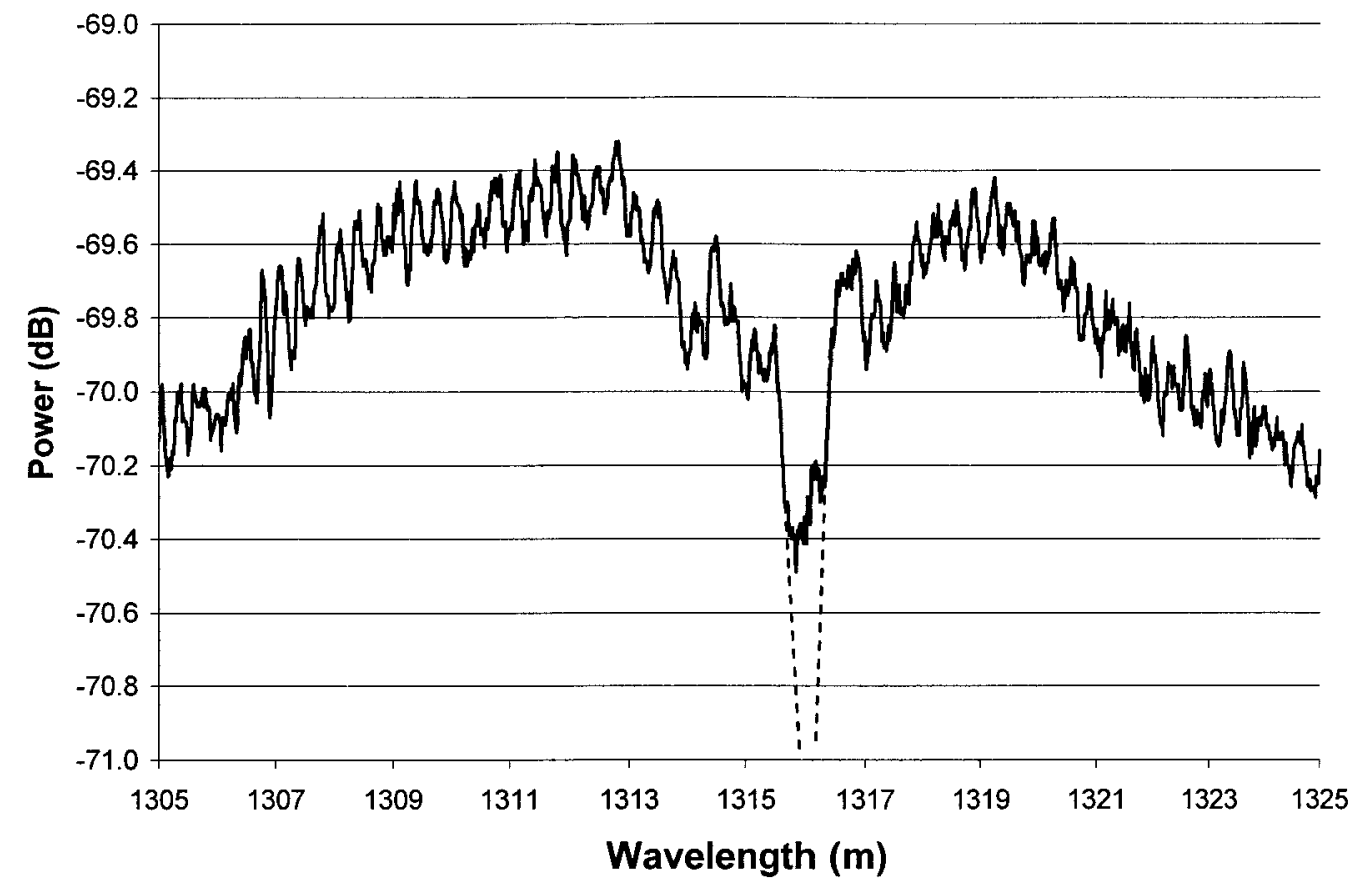

Figure 6.47. $20 \mathrm{~nm}$ resolution scan of post-bump Bragg wavelength plot at $146.0 \mathrm{~mA}$.

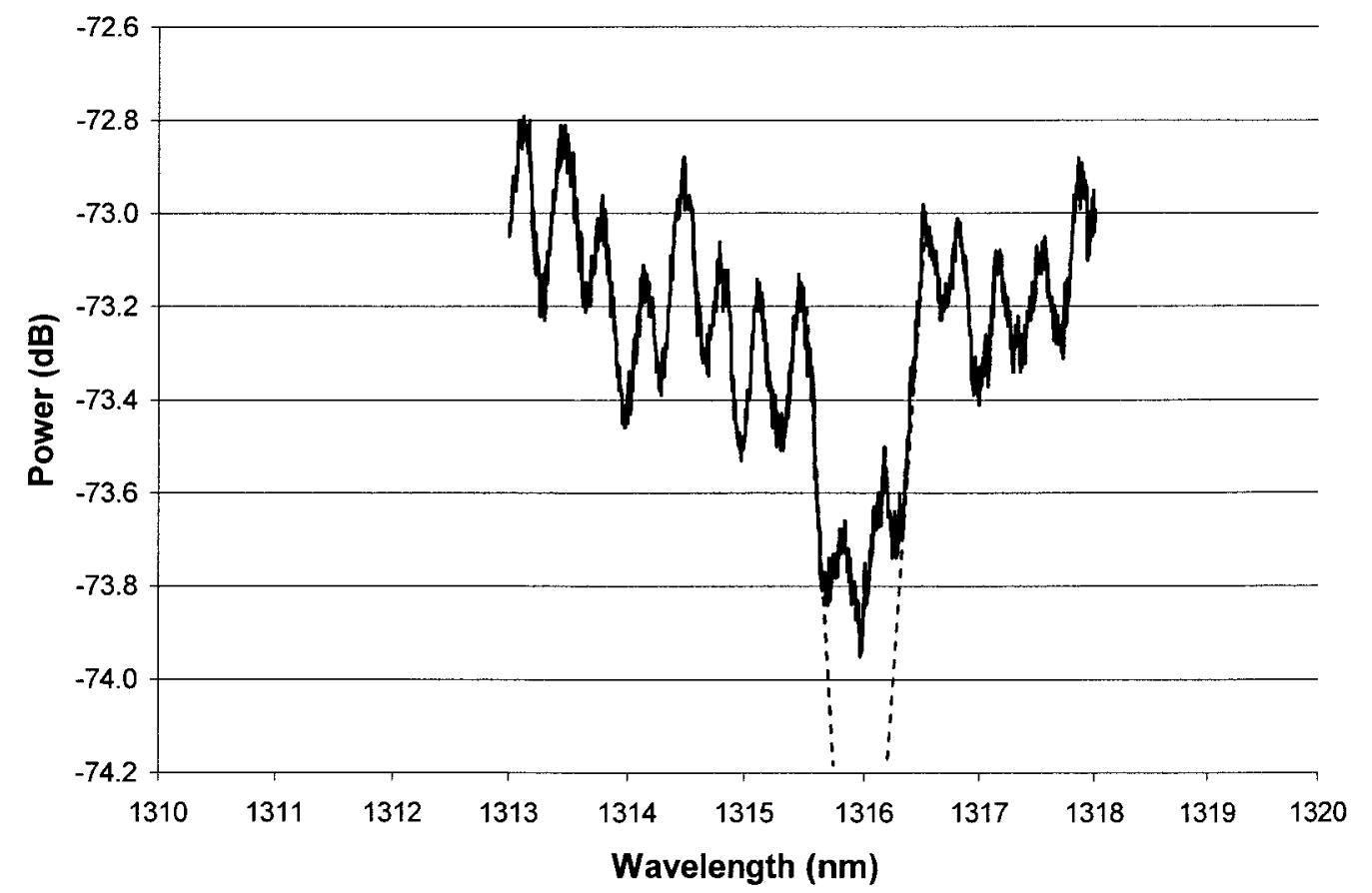

Figure 6.48. $5 \mathrm{~nm}$ resolution scan of post-bump Bragg wavelength plot at $146.0 \mathrm{~mA}$. 


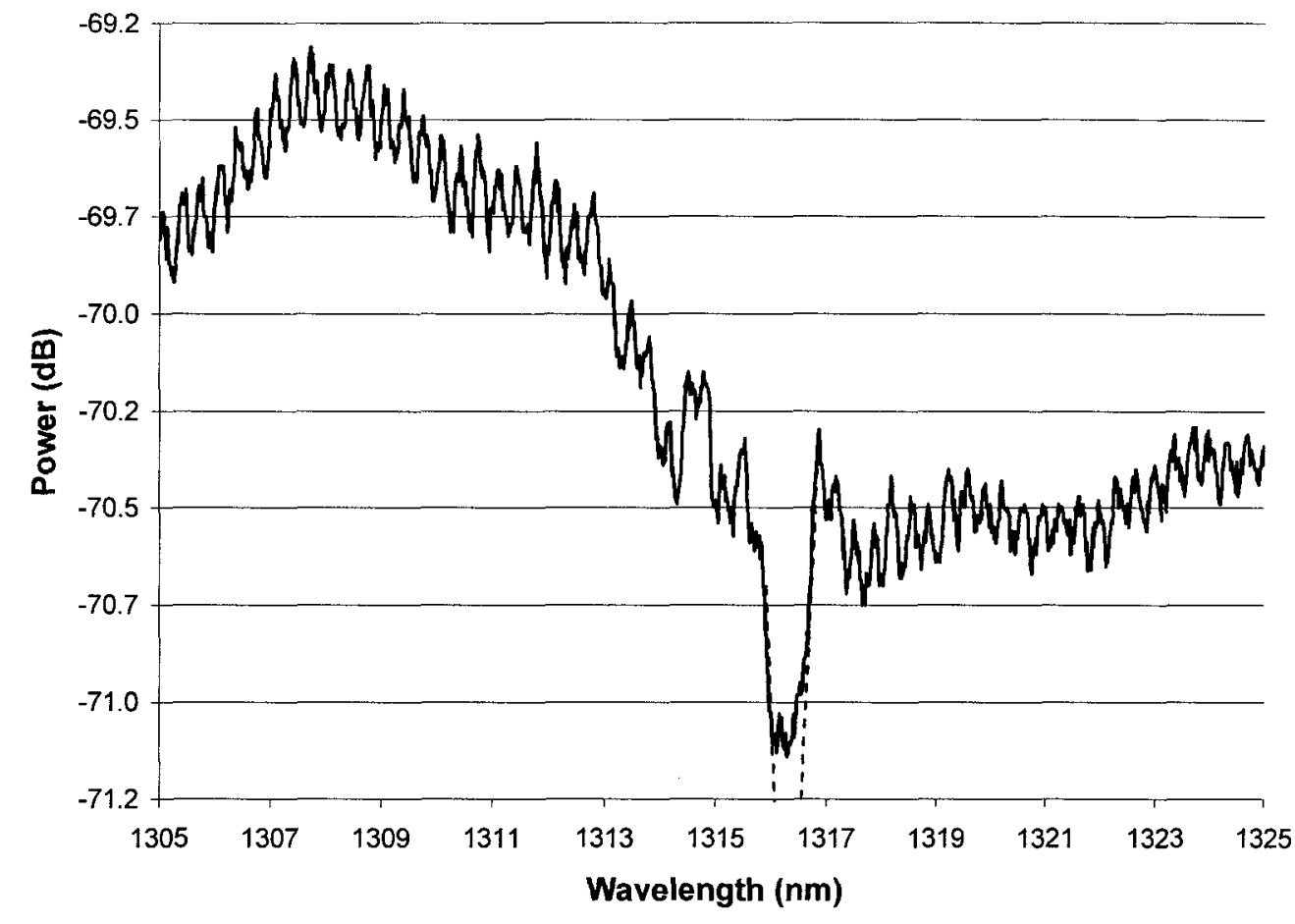

Figure 6.49. $20 \mathrm{~nm}$ resolution scan of pre-buckle Bragg wavelength plot at $153.0 \mathrm{~mA}$.

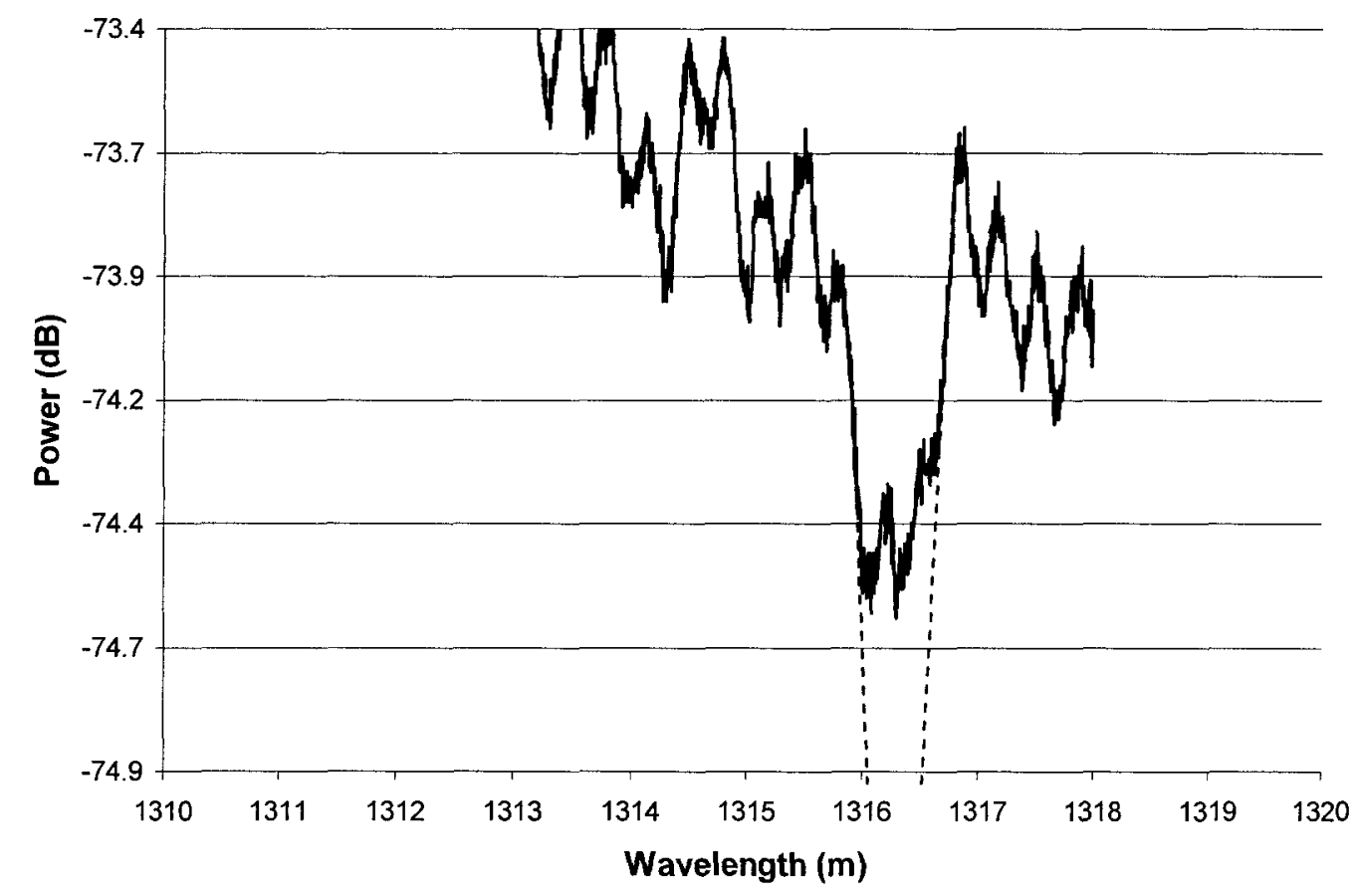

Figure 6.50. $5 \mathrm{~nm}$ resolution scan of pre-buckle Bragg wavelength plot at $153.0 \mathrm{~mA}$. 


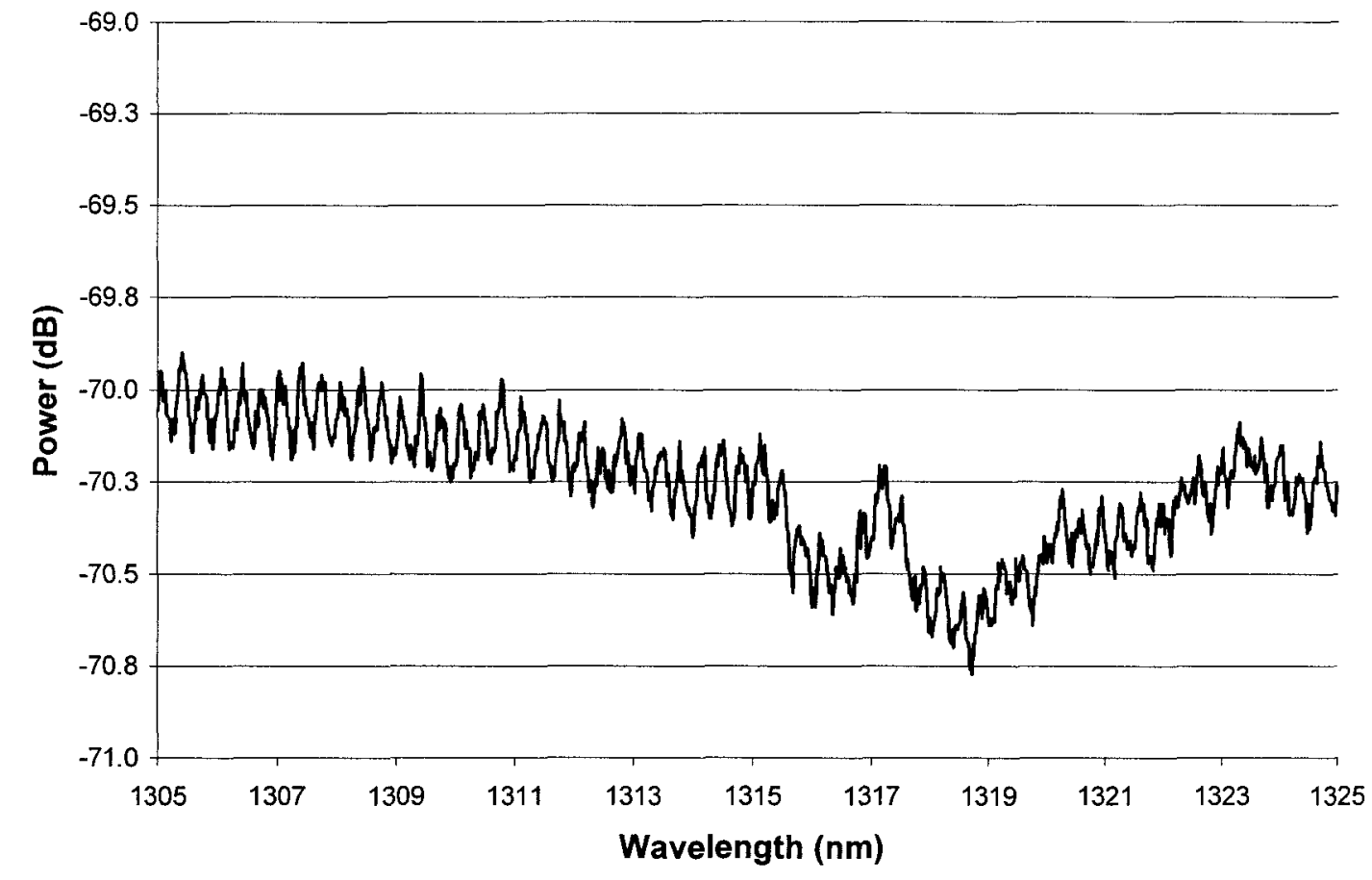

Figure 6.51. $20 \mathrm{~nm}$ resolution scan of post-buckle Bragg wavelength plot at $154.0 \mathrm{~mA}$.

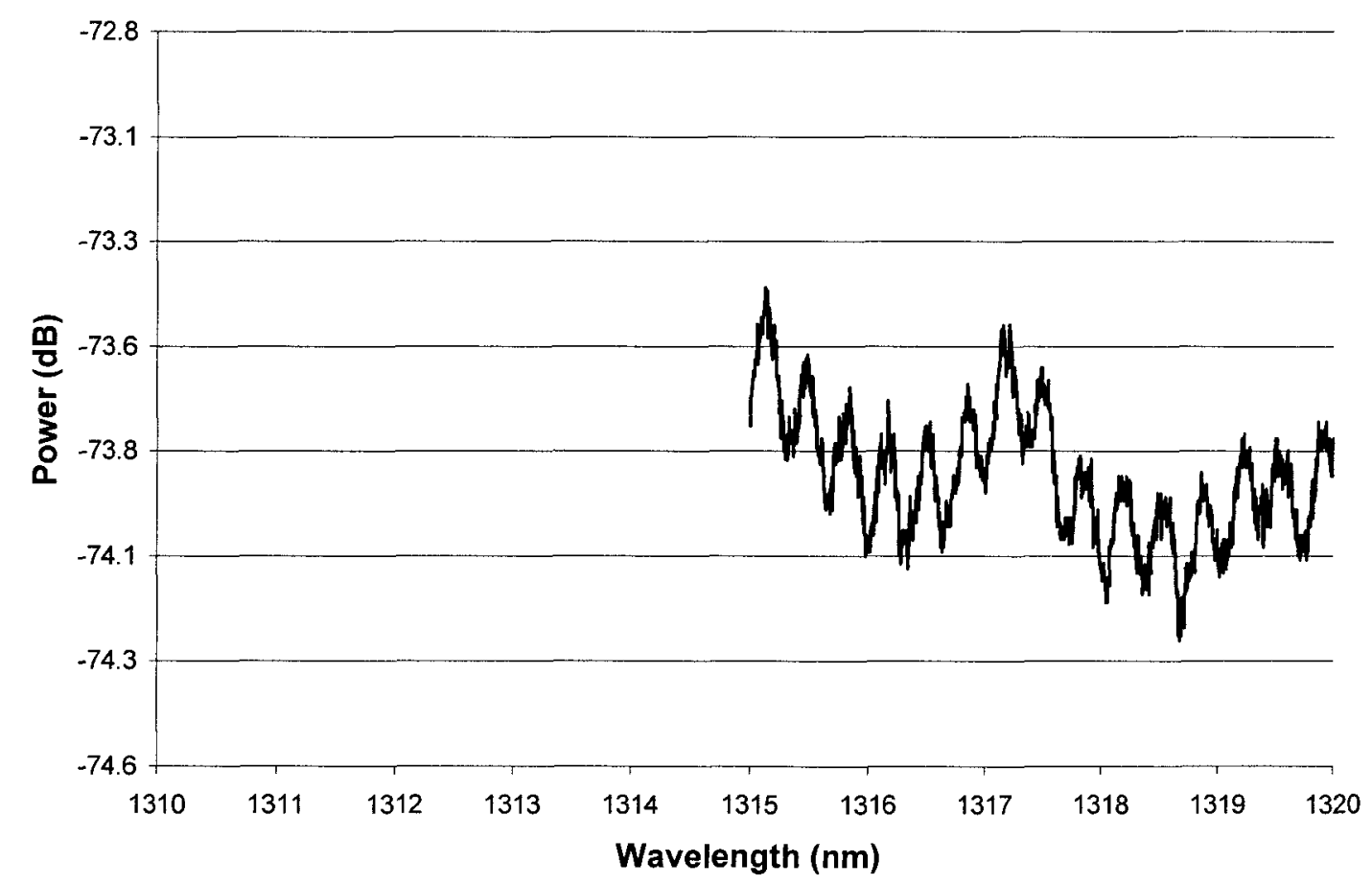

Figure 6.52. $5 \mathrm{~nm}$ resolution scan of post-buckle Bragg wavelength at $154.0 \mathrm{~mA}$. 
Figure 6.53 collects these critical Bragg dips into a single plot to show the comparative shift in the different operational regimes. The bottom of the dips were positioned to the same level for comparison. This figure is similar to the collection of critical thermo-mechanical deflection heights displayed in figure 6.20.

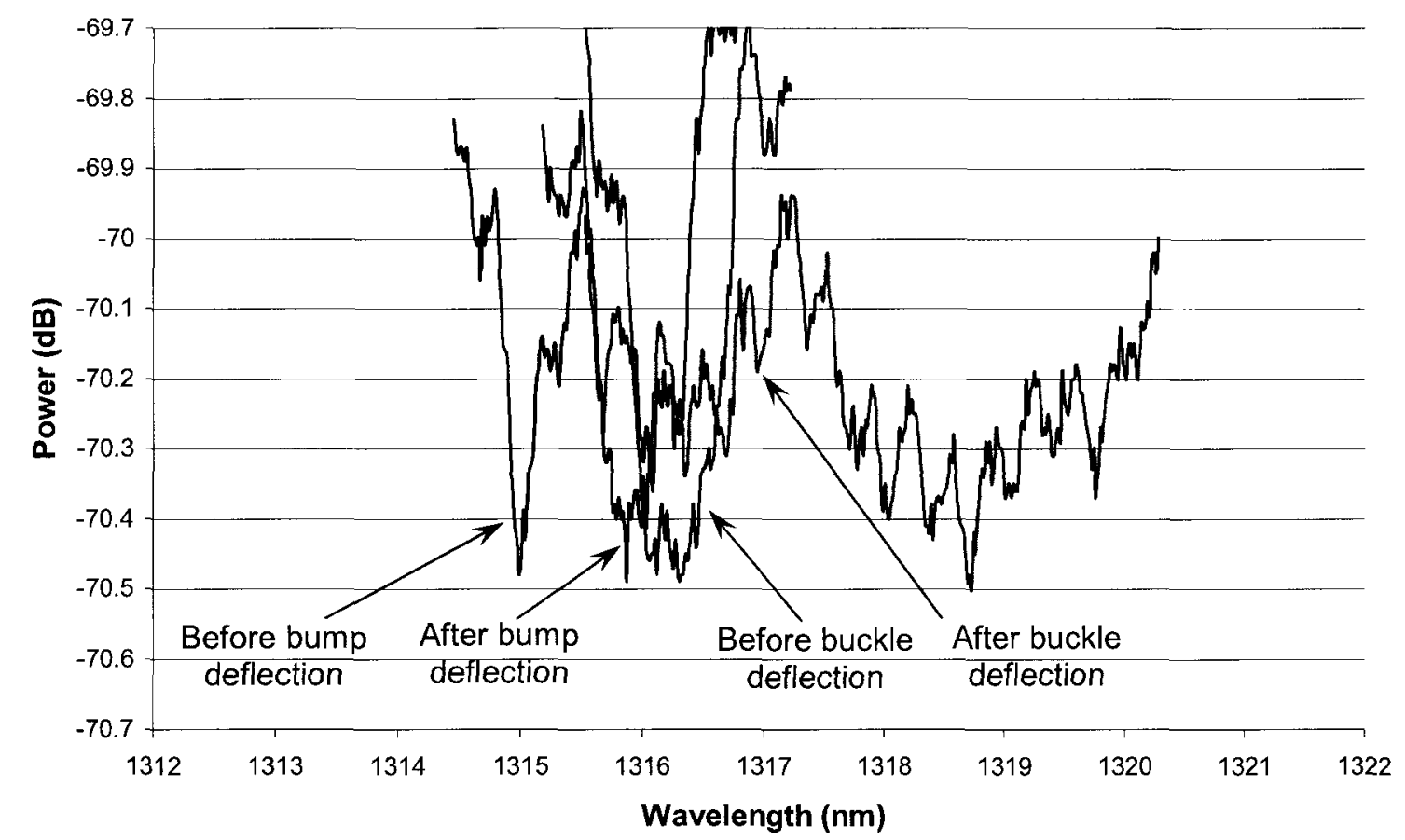

Figure 6.53. Plot of the Bragg filter wavelengths before and after bump deflection and before and after buckle deflection of the waveguide bridge filter.

Figure 6.54 plots the continuous thermo-mechanical shift of the Bragg wavelength in $0.9{ }^{\circ} \mathrm{C}$ increments along the forward and return path of a suspended rib waveguide filter. The experimental thermomechano-optical response of the D2-16 WG-1 device includes a pre-buckle thermal sensitivity of $76 \mathrm{pm} /{ }^{\circ} \mathrm{C}$, a bump shift of $0.39 \mathrm{~nm}$, a meta-stable thermal sensitivity of $62 \mathrm{pm} /{ }^{\circ} \mathrm{C}$, a buckle shift of $0.95 \mathrm{~nm}$, and a post-buckle thermal sensitivity of $88 \mathrm{pm} /{ }^{\circ} \mathrm{C}$. There is a $16 \%$ increase of the post-buckle sensitivity over the pre-buckle rate. The corresponding analytic rates calculated in section 4.4 and 
plotted in figure 4.11 include a pre-buckle thermal sensitivity of $83 \mathrm{pm} /{ }^{\circ} \mathrm{C}$, a buckle shift of $0.55 \mathrm{~nm}$, and a post-buckle thermal sensitivity of $99 \mathrm{pm} /{ }^{\circ} \mathrm{C}$. There is a $19 \%$ increase of the post-buckle sensitivity over the pre-buckle rate.

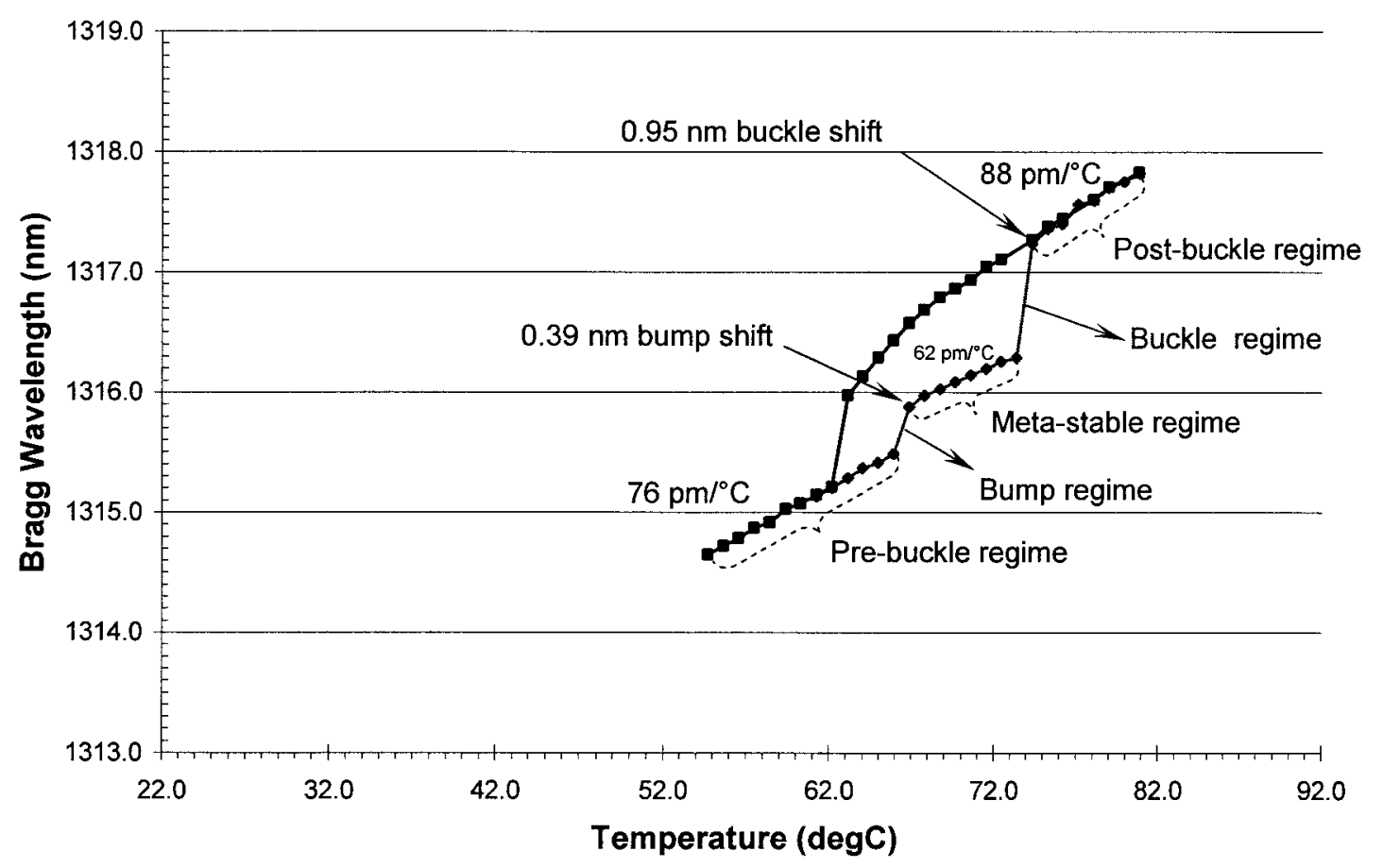

Figure 6.54. Thermomechano-optical response of the D2-16 WG-1 suspended waveguide filter.

The same bi-stable response that was plotted for the thermo-mechanical characterisation in figure 6.43 was observed in the thermomechano-optical response. Figure 6.54 shows a different return path for the Bragg filter wavelength as the device temperature is decreased from the post-buckle regime. The bi-stable Bragg filter wavelength difference was $0.95 \mathrm{~nm}$ at the beginning of the post-buckle regime and $0.77 \mathrm{~nm}$ before the waveguide bridge returned to the pre-buckle regime.

Bump and buckle shifts in the Bragg filter wavelength were measured for a second device, D2-20 WG-1, at $0.24 \mathrm{~nm}$ and $0.96 \mathrm{~nm}$ respectively, to compare to the 
D2-16 WG-1 results of $0.39 \mathrm{~nm}$ and $0.95 \mathrm{~nm}$. The extensive series of measurements required to determine the full thermomechano-optical response, as well as the corresponding thermo-mechanical buckle height measurements, were not conducted on D2-20.

The analytic model plotted in figure 4.11 does not take into account the metastable regime after the bump deflection, which was experimentally plotted in figure 6.54 . A second analytic model was prepared to take into account the effect the bump regime has on the thermomechano-optical response of the device. In this analysis the $0.5 \mu \mathrm{m}$ bump deflection releases the axial stress and allows the bridge to thermally expand, though not buckle. The thermal expansion occurs before the critical buckle temperature so the expansion is not enough to deflect the bridge to its buckling mode shape. The pitch of the Bragg grating expands according to the mechanical effect of thermal expansion and not according to the non-mechanical expansion of the buckling mode shape.

The bump temperature used in the analytic model to calculate the thermal expansion of the Bragg grating was determined experimentally to be $7.5^{\circ} \mathrm{C}$ before the critical buckle temperature. At the bump temperature the photo-elastic effect on $n_{\text {eff }}$, that had accumulated since room temperature, is released. Over the next $7.5^{\circ} \mathrm{C}$ thermal shift in which the bridge does not deform further, axial stress builds again, producing a photoelastic influence on $n_{\text {eff }}$ once more. The influence of the photo-elastic effect will be released a second time when the waveguide deflects $15.1 \mu \mathrm{m}$ to its buckling mode shape. At this deflection the spread of the Bragg grating pitch is due to the buckling mode shape of the waveguide bridge, and was previously calculated in section 4.4. The buckle shift 
in the Bragg wavelength smaller in this example since the grating pitch will have already been spread due to the bump shift. Figure 6.55 plots the result of these analytic calculations.

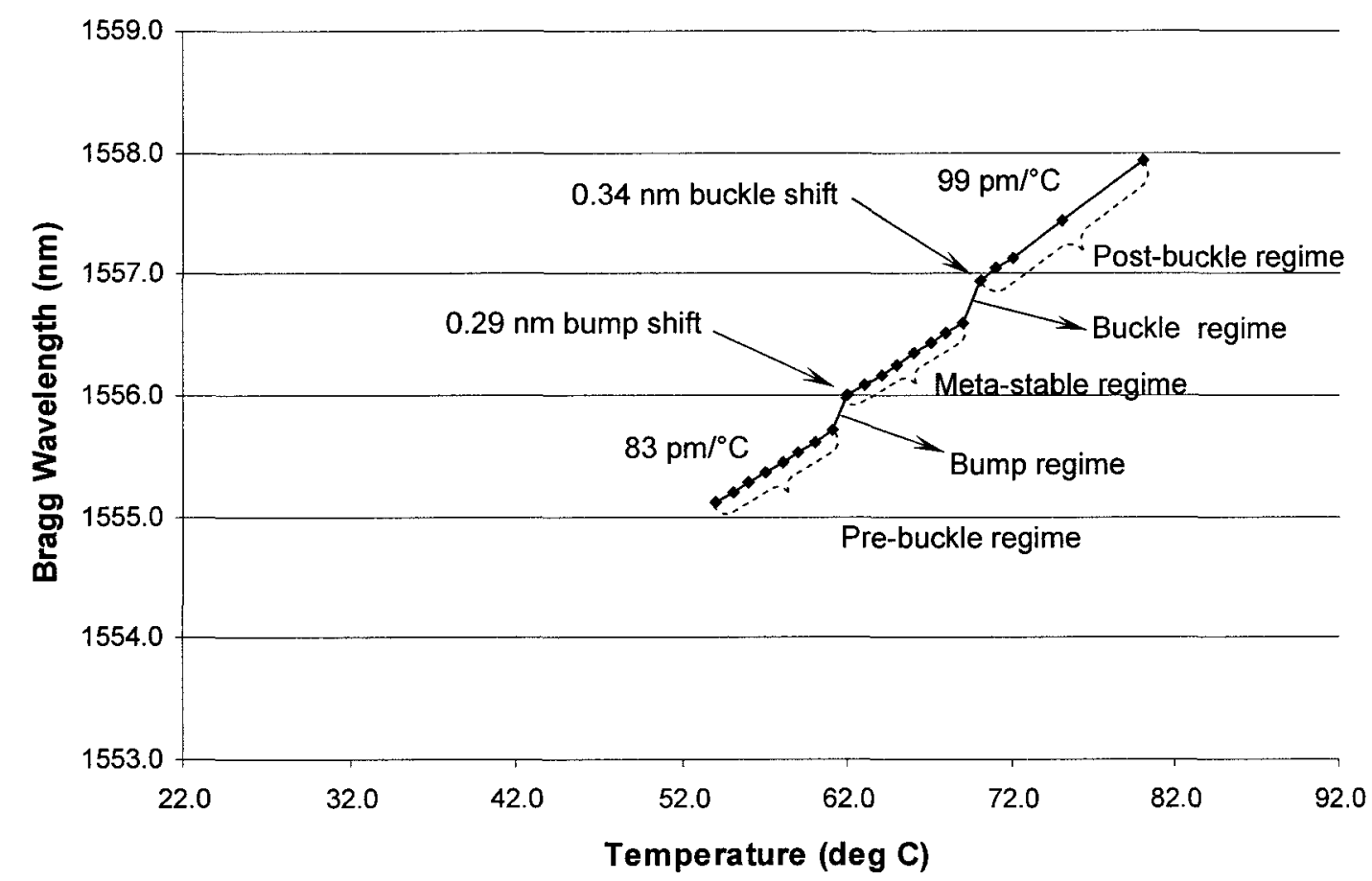

Figure 6.55. Theoretical thermomechano-optical response of device as a result of analysis over the pre-buckle, bump, buckle, and post-buckle regimes.

D2-16 WG-1 was thermo-mechanically characterised separately from the thermomechano-optical characterisation. It is useful to compare the thermo-mechanical buckle height plot to the thermomechano-optical Bragg filter wavelength plot to examine the consistency between the separate experiments. The comparison between the two plots is shown in figure 6.56 where the left axis depicts the Bragg wavelength and the right axis depicts the buckle height. As the temperature increases there is little difference between the temperature at which the bump and buckle effects occur in the plots. This shows a good degree of stability in the experimental setup. 
The comparison between these two experimental plots also shows that the temperature at which the bridge returns to its pre-buckle regime is slightly further apart, at $3.8^{\circ} \mathrm{C}$. On the return path of thermal actuation, when the magnitude of thermal strain is close to the deformation limit that keeps the bridge from being restored to its prebuckle regime, the slightest physical disturbance will cause the bridge to return a few degrees Celsius early. In another thermomechano-optical experimental run on the D2-16 device, in which the post-buckle Bragg filter wavelength was too weak to measure, the bridge waveguide returned to the pre-buckle regime at a temperature within $0.4^{\circ} \mathrm{C}$ of the thermo-mechanical temperature.

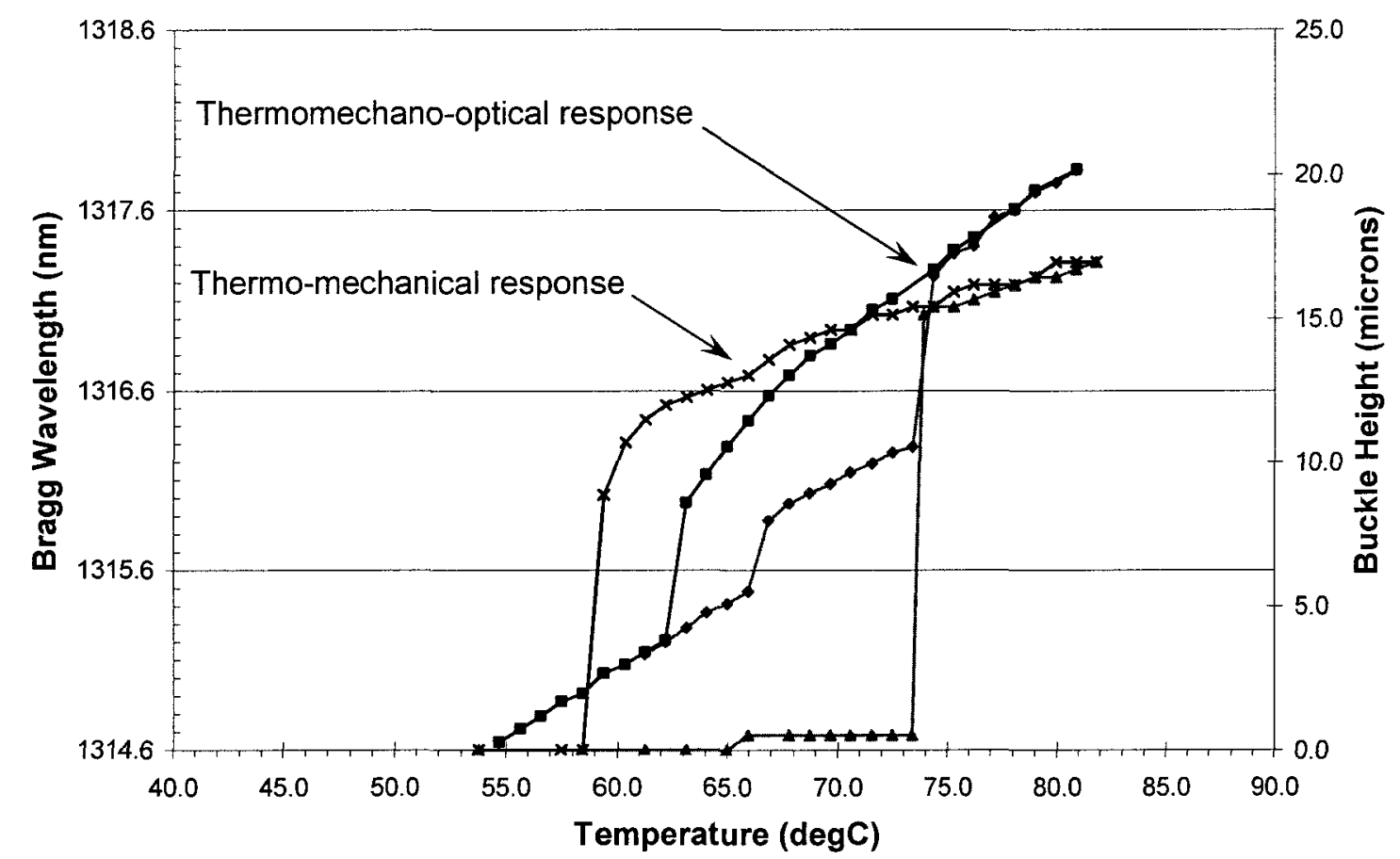

Figure 6.56. Overlay of thermomechano-optical and thermo-mechanical response of D2-16 WG-1.

The characterisation of thermo-mechanically tunable Bragg filters on SOI rib waveguide bridges has allowed the experimental validation of the analytic model for the 
thermomechano-optical behaviour of the device. The integration of a Bragg filter on a buckling waveguide bridge provides a discrete shift in the Bragg filter wavelength of $0.95 \mathrm{~nm}$ at the critical buckle temperature. The integration also provides a $16 \%$ increase in thermal sensitivity of the Bragg filter wavelength in the post-buckle regime. The meta-stability and bi-stability effects of the MOEMS device were also measured. The value of these additional performance characteristics would depend on the application the device was used for. 


\section{Conclusions}

\subsection{Summary of Thesis}

The purpose of this thesis was to investigate the consequence of coupling the material and structural elements of an actuator to an optical component integrated within that actuator. A device was proposed in which a Bragg grating filter was integrated within a thermally actuated waveguide bridge. A MOEMS based thermo-mechanically tunable Bragg grating filter on an SOI rib waveguide bridge was designed, fabricated, and characterised to verify that the thermal, mechanical and optical behaviour was in agreement with the predictions of the analytic model. The contributions that made this investigation possible were:

1) The development of a process flow to fabricate the device. The primary gates successfully completed in this process flow included Bragg filter integration into a rib waveguide; integration of these elements onto a fixed-fixed bridge that was released as a freestanding structure; integration of heaters on unreleased rib waveguide bridge filters; reliable waveguide facet preparation for optical characterisation; and prototype packaging of Bragg filters with integrated heaters on unreleased rib waveguide bridges $[101,120,121]$.

2) The development of a sacrificial bridge release post-process to produce up to $4000 \mu \mathrm{m}$ long, $5.0 \mu \mathrm{m}$ thick fixed-fixed waveguide bridges with a suspension of $2.0 \mu \mathrm{m}$ [122].

3) The measurement of an experimental buckle height of $15.1 \mu \mathrm{m}$, which corresponded to the calculation of a theoretical buckling mode height of $14.8 \mu \mathrm{m}$, for a 
$2000 \mu \mathrm{m}$ long, $5.0 \mu \mathrm{m}$ thick rib waveguide bridge [123].

4) The development of a prototype packaging post-process to allow measurement of the thermal sensitivity of a Bragg grating filter with integrated heaters in the pre-buckle regime. The experimental pre-buckle thermal sensitivity measured $76 \mathrm{pm} /{ }^{\circ} \mathrm{C}$, while the theoretical value was $83 \mathrm{pm} /{ }^{\circ} \mathrm{C}[124,125]$. These measurements agreed with literature for externally heated Bragg grating filters on SOI rib waveguides. This contribution also demonstrated a technique for indirectly measuring the thermal sensitivity of a Bragg filter by mapping an integrated heater thermal tuning rate to an external heater LD current thermal tuning rate.

According to theoretical analysis, the thermo-optic effect on $\Delta n_{e f f}$ was responsible for more than $99 \%$ of the $83 \mathrm{pm} /{ }^{\circ} \mathrm{C}$ pre-buckle Bragg filter sensitivity. The remaining influence on $\Delta n_{\text {eff }}$ came from the photo-elastic effect. This is an example of a thermally tuned device that does not take full advantage of coupling material and structural effects to its optical performance.

5) The measurement of an experimental Bragg filter wavelength shift, $\Delta \lambda_{B}=0.95 \mathrm{~nm}$, caused by the waveguide bridge deflecting to its buckling mode shape at the critical buckle temperature. This abrupt shift in the Bragg filter wavelength between the pre- and post-buckle regimes was due to a combination of $\Delta n_{e f f}$ and $\Delta \Lambda$. The theoretical $\Delta \lambda_{B}$ value depended on the absence or presence of a bump deflection, which resulted in respective Bragg filter wavelength shifts of $0.55 \mathrm{~nm}$ and $0.34 \mathrm{~nm}$.

In an ideal device without bump deflection, modelled in $1{ }^{\circ} \mathrm{C}$ increments to follow the experimental conditions, the thermo-optic effect now only accounted for $72 \%$ of $\Delta n_{\text {eff. }}$ The release of the cumulative photo-elastic effect made up the other $28 \%$. By 
coupling the material effect of the buckled waveguide bridge to the Bragg filter response, the photo-elastic influence was increased from its negligible pre-buckle impact to over one quarter of the total $\Delta n_{\text {eff. }}$ Altogether, $\Delta n_{\text {eff }}$ accounted for $37 \%$ of the influence on $\Delta \lambda_{B}$ in the buckle regime. The majority of the shift in the Bragg filter wavelength was provided by the buckle expansion of the Bragg grating pitch, $\Delta \Lambda$. This was a clear example of the benefit of coupling the material and structural influence of an actuator to an integrated optical component.

6) The measurement of an experimental post-buckle thermal sensitivity of $88 \mathrm{pm} /{ }^{\circ} \mathrm{C}$, which corresponded to a theoretical value of $99 \mathrm{pm} /{ }^{\circ} \mathrm{C}$. According to theoretical analysis, the thermo-optic influence on $\Delta n_{\text {eff }}$ was responsible for $90 \%$ of the $99 \mathrm{pm} /{ }^{\circ} \mathrm{C}$ post-buckle Bragg filter sensitivity. The remaining $10 \%$ came from a spread of the Bragg grating that was the result of continued out-of-plane deflection of the waveguide bridge due to thermo-mechanical expansion.

Since the sensitivity of the pre-buckle Bragg filter wavelength was dominated by the thermo-optic effect, and this effect remained through out the entire pre- to post-buckle range of operation, the influence of the thermo-mechanical expansion on the Bragg filter sensitivity could be experimentally isolated in the post-buckle regime. A $16 \%$ increase in thermal sensitivity was gained from integrating the Bragg grating filter described in 4) into a thermo-mechanically actuated fixed-fixed rib waveguide bridge. The theoretical increase was $19 \%$.

While an increase in thermal sensitivity could also be obtained from unconstrained linear thermal expansion, the buckling mode shape of the thermomechanical actuator provided a greater spread in the Bragg grating pitch per degree 
Celsius. The thermal sensitivity of the device was increased by coupling the structural effect of the buckling mode profile to the integrated Bragg grating.

Meta-stability and bi-stability in the Bragg filter response were also observed over the course of characterising the device. Experimentation showed that the bump deflection which began the meta-stable regime was only observed in beams with a "T" cross-section, not in beams with the more common rectangular (or prismatic) crosssection. It is possible that this meta-stable regime, which exists between the pre- and post-buckle regime, is a superposition of the buckling mechanics of the larger slab beam and the smaller rib beam on top of it.

Bump deflection caused the bridge to move one tenth the bridge thickness out-ofplane, or $0.5 \mu \mathrm{m}$, where it remained mechanically stable for a thermal shift of $7.5^{\circ} \mathrm{C}$ until the critical buckle temperature. Whereupon the bridge deflected through to a buckling mode height of $15.1 \mu \mathrm{m}$. Bump deflection caused a spread in the Bragg grating pitch that was likely the direct result of linear (axial) thermo-mechanical expansion, and not buckling mode deflection, since the bump occurred before the critical buckle temperature. The Bragg filter wavelength shift resulting from the bump deflection was measured to be $0.39 \mathrm{~nm}$ and calculated to be $0.29 \mathrm{~nm}$.

The cumulative influence of the photo-elastic effect at the critical buckle temperature was reduced as a result of the bump deflection, which released the axial stress on the waveguide bridge. The photo-elastic effect on $n_{\text {eff }}$ would begin to accumulate again in this meta-stable state until the critical buckling temperature occurred, but this was only an increase of $7.5^{\circ} \mathrm{C}$ from the bump temperature. The thermal sensitivity of the Bragg filter wavelength in the meta-stable regime was measured to be 
$62 \mathrm{pm} /{ }^{\circ} \mathrm{C}$.

Optical bi-stability was observed in the Bragg filter response from the start of the post-buckle regime along the return to the pre-buckle regime. In this bi-stable regime there would be two possible Bragg wavelengths for a given temperature, the value of which would depend on whether the device was in a forward or return path. The bistable regime occurred over a span of $15{ }^{\circ} \mathrm{C}$. The Bragg wavelength difference at the start of the post-buckle regime was $0.95 \mathrm{~nm}$, while the Bragg wavelength difference at the return to the pre-buckle regime was $0.77 \mathrm{~nm}$.

\subsection{Future Work}

Future work relating to improvement of the device design and fabrication process will be divided into thermal, mechanical, and optical development. The primary improvement to the thermal component of the device would be made by producing an integrated heater that could withstand the bridge release process. The current method of depositing a thin film heater on the surface of the bridge waveguide is vulnerable to delamination in the release process. A resistive element could be formed within the bridge, adjacent to the rib waveguide, through ion implantation and diffusion. The resulting semiconductor heater would not attenuate the optical signal propagating along the waveguide since the rib would contain the mode within the undoped silicon. Doping the silicon to create the heater strips would not change the mechanical properties of the waveguide bridge in relation to the buckling mechanics.

Mechanical improvement to the device design and fabrication could be made by releasing waveguide bridges with an aspect ratio greater than the 400:1 ratio the current devices have. This would translate to a longer bridge to increase the Bragg grating 
length, or a thinner bridge to reduce the waveguide cross-section. This could be accomplished with the current bridge release process by ensuring the sacrificial thermal oxide was grown on the handle layer of the SOI. This would result in an "A" sacrificial oxide etch profile that would reduce HF etch time and provide built-in support for the released waveguide bridge.

Optical improvement of the Bragg filter response could be made in a number of ways. A shallower grating would ensure more of the energy from the optical mode remains in the rib portion where the filter is. A mask in which the grating width matches the waveguide width would ensure a single Bragg grating duty cycle for the optical mode so that the strength of the Bragg response was not spread over two gratings with the same pitch but different duty cycle.

The grating was placed along the centre half of the waveguide bridge for this research. This produced a Bragg dip in the post-buckle regime that had a large $\delta_{3 d B}$ bandwidth. The post-buckle Bragg dip bandwidth could be reduced, and its buckle shift increased, by centring the grating along the area of the bridge that had the largest uniform local spread, even if it meant reducing the overall length of the grating.

COMSOL is a candidate to refine future design development. With its multiphysics module, the software can couple the photo-elastic and thermo-optic effects of the filter response over the pre-buckle regime of operation. Similarly, the software could couple the change in Bragg grating pitch from expansion of the actuating waveguide with the thermo-optic response in the post-buckle regime. Combining the results would provide a spectral response for the device operating over the entire pre- to post-buckle regime. The software would also be useful to explore the nature of the bump 
deflection and meta-stable regime, and how it relates to the cross-section of the waveguide bridge.

\subsection{Applications}

An important feature in the functionality of an optical device is tunability. A tunable filter allows a reduction in the number of components in a system such as an optical switch. A reduction in components results in a reduction in insertion loss and power requirements, and an overall lower system cost. These benefits can be enhanced if the material and structural effects on device performance can be coupled constructively.

The buckling mode shift in the Bragg filter wavelength from pre- to post-buckle regimes provides a mechano-optical separation of two $50 \mathrm{GHz}(0.4 \mathrm{~nm})$ optical channels. A dense wavelength optical system that was sensitive to interchannel cross-talk would benefit from the channel isolation offered by this device. The post-buckle regime saw a $16 \%$ increase in Bragg filter wavelength thermal sensitivity due to the buckling mode expansion of $\Lambda$. This would reduce the power required to tune the Bragg filter wavelength over a given bandwidth. The bistable nature of the device also suggests the possibility of a mechano-optical based flip-flop memory element.

A variation on the distributed feedback laser originally shown in figure 1.8 would be possible by using an optically active material instead of silicon for the bridge waveguide material. The Bragg grating would be separated so that an active cavity was formed in the centre of the suspended waveguide bridge.

Controlling strain is an important factor in quantum dot engineering. The strain along the length of a buckled waveguide bridge changes from the anchor point, where it is zero; to a maximum at the bridge inflection point. Quantum dots placed on top of the 
waveguide rib, that run the length of the bridge, could make use of the gradation of strain to generate a change in the spectral response of the dots.

Strain control is also an important factor in the technique of manipulating the band offset of indirect bandgap materials such as silicon and germanium. The desired strain on the crystal lattice can be achieved by allowing a released structure to permanently collapse to its handle substrate [126]. If strain based band offset manipulation were possible in the free-standing bridge waveguide structures presented here, thermo-mechanical strain tuning in the post-buckle regime would allow a greater degree of bandgap control. 


\section{Appendices}

\section{Appendix I: Verifying Critical Buckle Stress Calculations}

In order to validate equations 3.22 and 3.47 , the self-buckling behaviour of a fixed-fixed polysilicon micromachined beam under resistive heating, reported by $M$. Chiao and L. Lin [127], was used. While the optical properties of polysilicon are not similar to single crystal silicon, the mechanical properties such as the coefficient of thermal expansion and Young's Modulus are. Given a fixed-fixed bridge structure $2 \mu \mathrm{m}$ wide, $2 \mu \mathrm{m}$ thick and $100 \mu \mathrm{m}$ long; they found that a beam temperature of $531^{\circ} \mathrm{C}$ was required to cause the compressive stress necessary for the beam to buckle.

$$
\begin{array}{ll}
\sigma_{c r}=\alpha \Delta T E & 3.22 \\
P_{c r}=\frac{4 \pi^{2} E I}{L^{2}} & 3.47
\end{array}
$$

The following analysis uses equation 3.22 to calculate the critical buckle stress using the material property coefficients they reported of $\mathrm{E}=150 \mathrm{GPa}$ and $\alpha=2.6 \times 10^{-6}{ }^{\circ} \mathrm{C}^{-1}$; and the critical buckle temperature they reported of $T_{c r}=531{ }^{\circ} \mathrm{C}$. This value is compared to the critical buckle stress calculated from equation 3.47. The results were in close agreement.

From Chiao's numbers and equation 3.22:

$$
\begin{aligned}
\sigma_{c r} & =\alpha \Delta T E \\
\sigma_{c r} & =\left(2.6 \times 10^{-6}{ }^{\circ} \mathrm{C}^{-1}\right)(531-22)^{\circ} \mathrm{C}\left(150 \times 10^{9} \mathrm{~N} / \mathrm{m}^{2}\right) \\
& =-198.5 \times 10^{6} \mathrm{~N} / \mathrm{m}^{2}
\end{aligned}
$$


where negative means compressive stress. From equation 3.47 with a beam length $L=$ $100 \mu \mathrm{m}$, width $w=2 \mu \mathrm{m}$, and thickness $t=2 \mu \mathrm{m}$.

$$
\begin{aligned}
P_{c r} & =\frac{4 \pi^{2} E I}{L^{2}} \\
& =7.896 \times 10^{-4} \mathrm{~N} \\
\sigma_{c r} & =P_{c r} / A \\
& =-197.4 \times 10^{6} \mathrm{~N} / \mathrm{m}^{2}
\end{aligned}
$$

With $T_{c r}=531{ }^{\circ} \mathrm{C}$, the thermal strain from $\varepsilon_{T}=\alpha \Delta T$ at the critical buckle temperature is $\varepsilon_{T}=1.316 \times 10^{-3}$. Recalling that $\sigma=E \varepsilon$, equations 3.47 and 3.42 can be combined to express the critical strain in the waveguide that must occur via thermal expansion for buckling to occur as:

$$
\varepsilon_{c r}=\frac{4 \pi^{2} t^{2}}{12 L^{2}}
$$

Using this equation, the strain at the critical buckle temperature can be independently calculated to be $\varepsilon_{c r}=1.323 \times 10^{-3}$, providing further validation of the relationships for the thermo-mechanical behaviour of a fixed-fixed beam.

There are some other useful observations that can be taken from equation 0.1 . The first is that the critical buckle strain, and therefore the critical buckle pressure, is not influenced by the modulus of elasticity, E. The next observation is that while the width of the waveguide will have no effect on the critical buckling pressure (or temperature), the length and thickness both do. Decreasing the length or increasing the thickness of the waveguide will increase the critical buckle pressure and therefore the critical buckle temperature. This fact is crucial to consider when designing the operational envelope of the waveguide bridge filter. The higher the $T_{c r}$, the greater the Bragg wavelength shift 
upon buckling. In addition, as the critical temperature increases there is more flexibility in the design process to maximize the performance of other design parameters such as the bandwidth at FWHM, $\delta \lambda_{3 d B}, \Delta \lambda_{B}$, and the channel isolation. 


\section{Appendix II: Run Sheets}

This appendix contains the run sheet of the process flow for the D2 version of the MOEMS device including post-process facet preparation, bridge release, and packaging.

\section{1) Bragg grating mask EBL (process steps performed at NRC-IMS)}

- RCA clean on SOl wafer

- Dehydration bake and HMDS spin-on

- Spin on ELIONIX ZEP520A positive e-beam resist

- Expose Bragg grating mask

- Develop using o-Xylene.

\section{2) Bragg grating etch}

- Singulate or cleave SOI wafer into $7 \mathrm{~mm} \times 12 \mathrm{~mm}$ die

- Descum die in Technics: base pressure $=4 \mathrm{~Pa}, \mathrm{O}_{2}$ pressure $=40 \mathrm{~Pa}$, ionization power $=100 \mathrm{~W}$, etch time $=10$ seconds.

- Mount four die on the centre of a $100 \mathrm{~mm}$ backing wafer using double sided carbon tape.

- Execute 5 minute conditioning run on ECR.

- Etch Bragg grating in ECR: base pressure $=2.4 \times 10^{-4} \mathrm{~Pa}$, wafer temp $=-30{ }^{\circ} \mathrm{C}$, $\mathrm{SF} 6$ flow $=3.65 \mathrm{sccm}, \mathrm{O}_{2}$ flow $=2.21 \mathrm{sccm}$, gas pressure $=0.8 \mathrm{~Pa}$, forward microwave power $=310 \mathrm{~W}, \mathrm{RF}$ bias $=10 \mathrm{~W}$, etch time $=1$ to 2 minutes.

- Detach die from backing wafer by heating wafer on hotplate at $80^{\circ} \mathrm{C}$ and gently prying the die off.

- Ash remaining grating mask in PlasmaPreen: isolate die from water cooled base with ceramic stand-offs. Base pressure $=4.0 \mathrm{~Pa}, \mathrm{O}_{2}$ pressure $=670 \mathrm{~Pa}$, etch time $=$ two runs of 5 minutes.

\section{3) Rib Waveguide Lithography}

- Dehydrate and clean die in PlasmaPreen: isolate die from water cooled base with ceramic stand-offs. Base pressure $=4.0 \mathrm{~Pa}, \mathrm{O}_{2}$ pressure $=670 \mathrm{~Pa}$, etch time $=5$ 
minutes.

- Spin coat HMDS, 30 seconds at $4000 \mathrm{rpm}$.

- Bake 60 seconds at $105^{\circ} \mathrm{C}$.

- Spin coat $S 1811,30$ seconds at $4000 \mathrm{rpm}$.

- Soft bake 60 seconds at $105^{\circ} \mathrm{C}$.

- Expose waveguide pattern for 16 seconds under vacuum contact (HP) mode.

- Develop for 60 seconds in MF321.

- Rinse in DI for 45 seconds.

- Blow dry with nitrogen and inspect.

- Further develop in MF321 if necessary.

- Passing inspection, hard bake 3 minutes at $125^{\circ} \mathrm{C}$.

\section{4) Rib Waveguide Etch}

- If the die sat over-night, another hard bake of 2 minutes at $125^{\circ} \mathrm{C}$ is performed.

- Descum die in Technics: base pressure $=4 \mathrm{~Pa}, \mathrm{O}_{2}$ pressure $=40 \mathrm{~Pa}$, ionization power $=100 \mathrm{~W}$, etch time $=60$ seconds.

- Perform a 15 second BOE on die.

- Mount die on the centre of a $100 \mathrm{~mm}$ backing wafer using double sided carbon tape.

- Execute 5 minute conditioning run on ECR.

- Etch rib waveguide in ECR: base pressure $=2.4 \times 10^{-4} \mathrm{~Pa}$, wafer temp $=-30{ }^{\circ} \mathrm{C}$, SF6 flow $=3.65 \mathrm{sccm}, \mathrm{O}_{2}$ flow $=2.21 \mathrm{sccm}$, gas pressure $=0.8 \mathrm{~Pa}$, forward microwave power $=310 \mathrm{~W}, \mathrm{RF}$ bias $=10 \mathrm{~W}$, etch time $=2$ to 3 minutes.

- Detach die from backing wafer by heating wafer on hotplate at $80^{\circ} \mathrm{C}$ and gently prying the die off.

- Ash remaining waveguide mask in PlasmaPreen: isolate die from water cooled base with ceramic stand-offs. Base pressure $=4.0 \mathrm{~Pa}, \mathrm{O}_{2}$ pressure $=670 \mathrm{~Pa}$, etch time $=$ two runs of 5 minutes.

\section{5) Heater Lithography (D2)}

- Dehydrate and clean die in PlasmaPreen: isolate die from water cooled base with ceramic stand-offs. Base pressure $=4.0 \mathrm{~Pa}, \mathrm{O}_{2}$ pressure $=670 \mathrm{~Pa}$, etch time $=5$ 
minutes.

- Spin coat HMDS, 30 seconds at $1500 \mathrm{rpm}$.

- Bake 60 seconds at $105^{\circ} \mathrm{C}$.

- Spin coat LOR3A, 30 seconds at $1500 \mathrm{rpm}$.

- Soft bake 5 minutes at $160^{\circ} \mathrm{C}$.

- Spin coat $S 1811,30$ seconds at $4000 \mathrm{rpm}$.

- Soft bake 60 seconds at $105^{\circ} \mathrm{C}$.

- Expose waveguide pattern for 26 seconds under soft contact (ST) mode.

- Develop for 60 seconds in MF321.

- Rinse in DI for 45 seconds.

- Blow dry with nitrogen and inspect.

- Further develop in MF321 if necessary.

6) Heater Evaporation Deposition (D2)

- Cover platform with aluminum foil and wipe down with IPA.

- Affix die to platform using magnets.

- Load platform into deposition chamber and pump down to $4.0 \times 10^{-5} \mathrm{~Pa}$

- Evaporate a titanium adhesion layer of $256 \AA$.

- Evaporate a gold layer of $9500 \AA$

\section{7) Heater Lift-Off (D2)}

- Preheat two beakers of Shipley Microposit Remover 1165 to $80^{\circ} \mathrm{C}$.

- Place die on Teflon holder and place holder in first Microposit beaker.

- Place beaker in heated water bath with ultra-sonic agitator

- If a substantial amount of time has passed without a lift-off, gently abrade surface of the die with a cotton tipped stick.

- When the LOR/PR/metal film has separated from the die, place die in second Microposit beaker.

- After 10 minutes remove die from second bath and rinse in DI.

- Blow dry with nitrogen and inspect. 


\section{8) Bridge Lithography}

- Dehydrate and clean die in PlasmaPreen: isolate die from water cooled base with ceramic stand-offs. Base pressure $=4.0 \mathrm{~Pa}, \mathrm{O}_{2}$ pressure $=670 \mathrm{~Pa}$, etch time $=5$ minutes.

- Spin coat HMDS, 30 seconds at $4000 \mathrm{rpm}$.

- Bake 60 seconds at $105^{\circ} \mathrm{C}$.

- Spin coat $\mathrm{S} 1811,30$ seconds at $4000 \mathrm{rpm}$.

- Soft bake 60 seconds at $105^{\circ} \mathrm{C}$.

- Expose waveguide pattern for 30 seconds under vacuum contact (HP) mode.

- Develop for 60 seconds in MF321.

- Rinse in DI for 45 seconds.

- Blow dry with nitrogen and inspect.

- Further develop in MF321 if necessary.

- Passing inspection, hard bake 3 minutes at $125^{\circ} \mathrm{C}$.

\section{9) Bridge Etch}

- If the die sat over night another hard bake of 2 minutes at $125^{\circ} \mathrm{C}$ is performed.

- Descum die in Technics: base pressure $=4 \mathrm{~Pa}, \mathrm{O}_{2}$ pressure $=40 \mathrm{~Pa}$, ionization power $=100 \mathrm{~W}$, etch time $=60$ seconds.

- Prepare a $100 \mathrm{~mm}$ backing wafer coated with PR with an open strip down the middle. Place strip of Kapton tape on the middle of the wafer, spin coat S1811, hard bake, and remove Kapton tape strip.

- Mount die on open strip of backing wafer using double sided carbon tape.

- Execute 5 minute conditioning run on ECR.

- Etch rib waveguide in ECR: base pressure $=2.4 \times 10^{-4} \mathrm{~Pa}$, wafer temp $=-30{ }^{\circ} \mathrm{C}$, SF6 flow $=3.65 \mathrm{sccm}, \mathrm{O}_{2}$ flow $=2.21 \mathrm{sccm}$, gas pressure $=0.8 \mathrm{~Pa}$, forward microwave power $=310 \mathrm{~W}, \mathrm{RF}$ bias $=10 \mathrm{~W}$, etch time $=6$ minutes

- Ensure the BOX layer has been exposed from silicon etch. Calibrate Nanospec to oxide and measure the oxide thickness at the bottom of the bridge etch.

- Upon successful inspection, detach die from backing wafer by heating wafer on hotplate at $80^{\circ} \mathrm{C}$, gently prying the die off. 
- Ash remaining bridge mask in PlasmaPreen: isolate die from water cooled base with ceramic stand-offs. Base pressure $=4.0 \mathrm{~Pa}, \mathrm{O}_{2}$ pressure $=670 \mathrm{~Pa}$, etch time $=$ two runs of 5 minutes.

\section{0) Waveguide Facet Cleave}

- Place die on single fab wipe sheet

- Butt glass slide edge against die, parallel to waveguides

- Butt second glass slide against the first so that the second slide forms a straight edge perpendicular to the waveguides.

- Draw scribe along edge of second slide

- Place a small diameter hex driver shaft under the die, aligned along the scribe

- Using the hex edges as a fulcrum, apply steady gentle pressure with index finger and thumb tips on either side of the scribe until die cleaves, exposing the waveguide facet.

\section{1) Bridge Release (30 $\mu \mathrm{m}$ wide bridge with $2 \mu \mathrm{m}$ BOX)}

- Place die flat on a Teflon platform and lower platform into conc. HF.

- After 17 minutes 30 seconds grip sides of the die parallel to the waveguides with flair nosed tweezers and remove.

- Alternately the Teflon platform can be removed from the conc. HF.

- Place die into a one litre Petri dish filled with DI water.

- After five minutes, remove die perpendicularly from the DI bath using flair nosed tweezers, gripping the edges of the die parallel to the waveguides.

- Place the die into a Petri dish filled with IPA for three minutes.

- Place the die into a Petri dish filled with HMDS for three minutes.

- Place the die into a Petri dish filled with acetone for three minutes.

- Remove the die from acetone and place the tip of an eyedropper holding PR along the side of one of the tweezer arms to allow a controlled flooding and acetone replacement with the PR

- Place the die in the centre of a $50 \mathrm{~mm}$ backing wafer. The PR will affix the die to the wafer.

- Place the backing wafer under a ventilated cover and place the entire arrangement 
under a fume hood for three days.

- Use a scalpel to remove the die from the backing wafer.

- Place the die on ceramic stand-offs within the reaction chamber of the PlasmaPreen. Base pressure $=4.0 \mathrm{~Pa}, \mathrm{O}_{2}$ pressure $=670 \mathrm{~Pa}$, etch time $=$ three runs of 5 minutes.

- Inspect the waveguide bridge for successful release using phase contrast illumination microscopy and the SEM.

\section{2) Die Packaging}

- Cleave $7 \mathrm{~mm} \times 7 \mathrm{~mm}$ shim spacer die to raise the waveguide facets of the device die above the surface of the chip carrier.

- Dispense a drop of Permatex "Permanent Strength Threadlocker" with a micropipette to adhere the shim to the DIP and the device die to the shim. Care should be taken to avoid excess adhesive that might squeeze out from the sides and wick down the waveguides.

- Cure adhesive over 12 hours on a hotplate set to $100^{\circ} \mathrm{C}$

- Mount package onto the heated workholder of a West-Bond 7400A ultrasonic wire bonder

- After determining the appropriate wire bonding power and temperature, wirebond the heaters to their respective pins. 


\section{Appendix III: Alternative Processes for Heater Protection during Bridge Release Post-process}

In addition to the process flow development described in section 5.3, two alternate strategies were explored to help the gold evaporated heaters survive the bridge release post-process. One strategy reduced the etch time in HF necessary to release the bridges. The other strategy protected the heaters during the etch release with a passivation layer of PR.

The first strategy made use of the MRC RIE-61 reactive ion etcher (RIE). This machine can handle various etch chemistries but is used primarily with $\mathrm{CHF}_{3}$ for its oxide etch capabilities. The SOI wafer used for device fabrication had a BOX "V" etch profile which meant the device layer/BOX interface etched more slowly than the BOX/handle layer interface. By dry etching the oxide to the handle layer of silicon before the sacrificial wet etch, the etch time would be reduced, potentially reducing the effect of delamination.

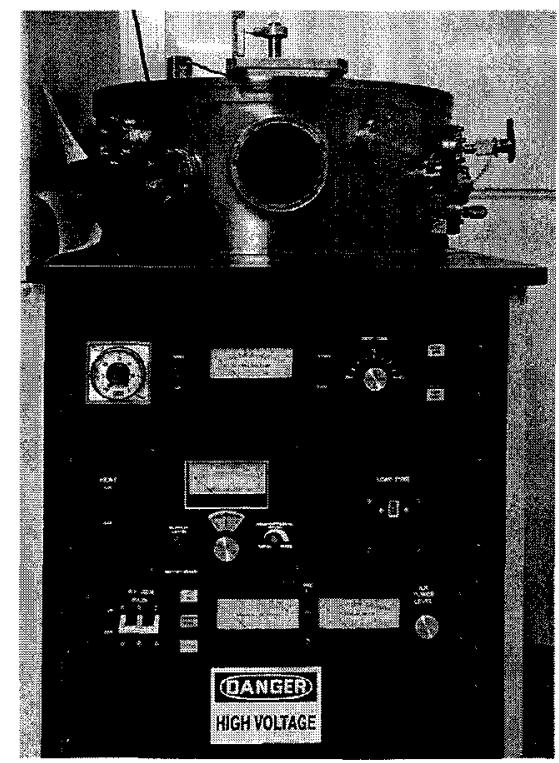

Figure 0.1. MRC RIE-51 Reactive Ion Etcher 
After the ECR etched the silicon to the BOX layer to define the bridge, the MRC would etch the buried oxide down to the handle layer silicon. Test etches are shown in figure 0.2 . By dry etching the exposed BOX, the bridge etch time for the "V" profile SOI was reduced from 17.5 minutes to 12 minutes.
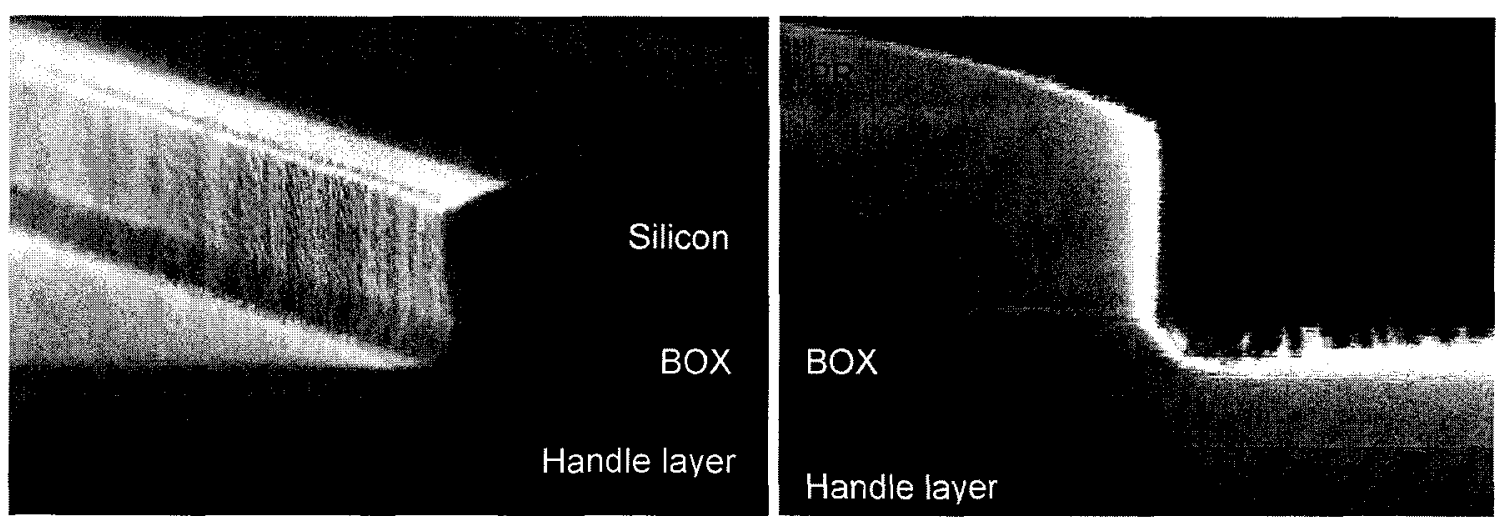

Figure 0.2. RIE results in which part of the BOX has been etched. The photoresist defining the bridge width has not yet been removed in these SEM cross-sections.

The second strategy involved passivating heaters with a film of PR. The passivation process was tested on two gold deposition runs, one with a Ti adhesion layer and one with a $\mathrm{Cr}$ adhesion layer. The passivation layer could be processed either by spin coating a layer of PR after the ECR bridge etch and re-exposing the bridge mask in the same position, or by spin coating a layer of PR that was retained after the ECR etch step for use in the MRC etch. The second method was superior since this passivating PR was self-aligned to the ECR bridge etch.

To assist the PR in its passivation role, an HMDS primer oven was used. This gave the PR much better adhesion to the native oxide on the silicon and greater resistance to penetration from the HF underneath as seen in figure 0.3 . The sample on the left saw only a spin coat of HMDS while the sample on the right was treated in the HMDS primer oven. 

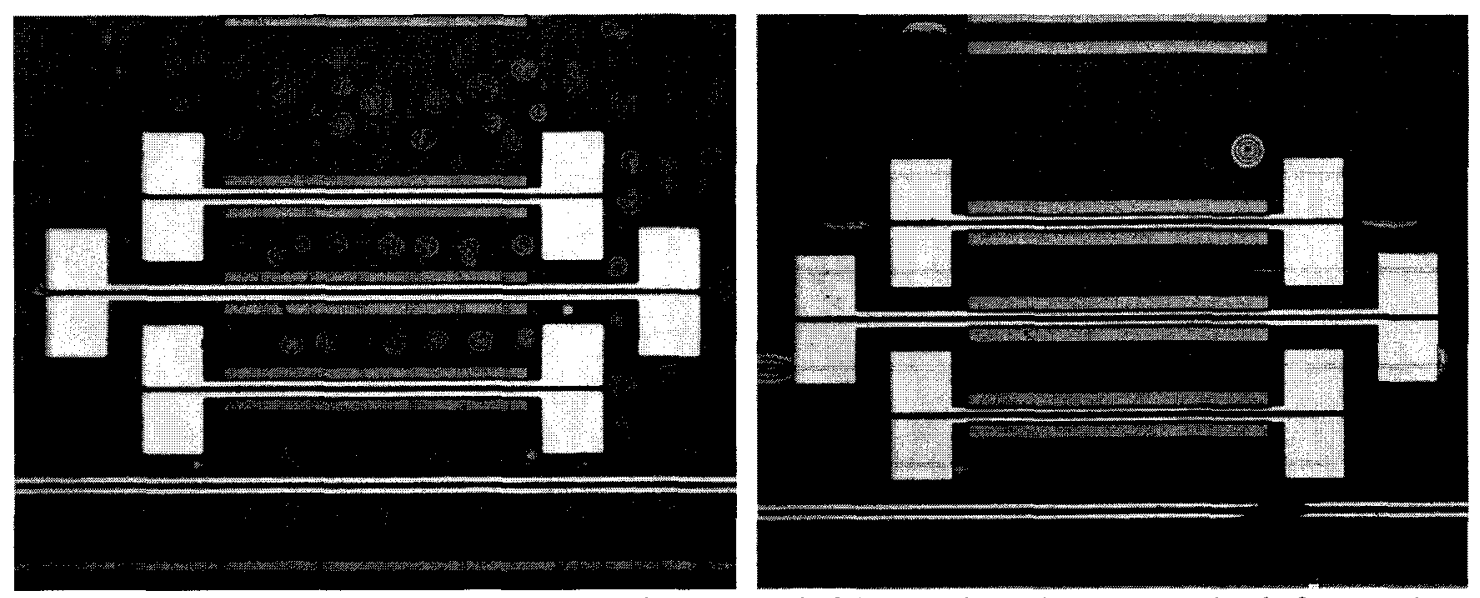

Figure 0.3. Both die have gone through the HF bridge etch. The PR on the left sample was processed with an HMDS spin coat. The PR on the right sample was processed in an HMDS primer oven.

Figure 0.4 shows results from PR passivation after the bridge release was complete and the PR was removed. The same $\mathrm{O} 2$ plasma that ashed the bridge support PR also removed the PR passivation layer. In trials, the Ti adhesion based heaters fared slightly better than the $\mathrm{Cr}$ adhesion based heaters, but substantial improvement was seen in neither.
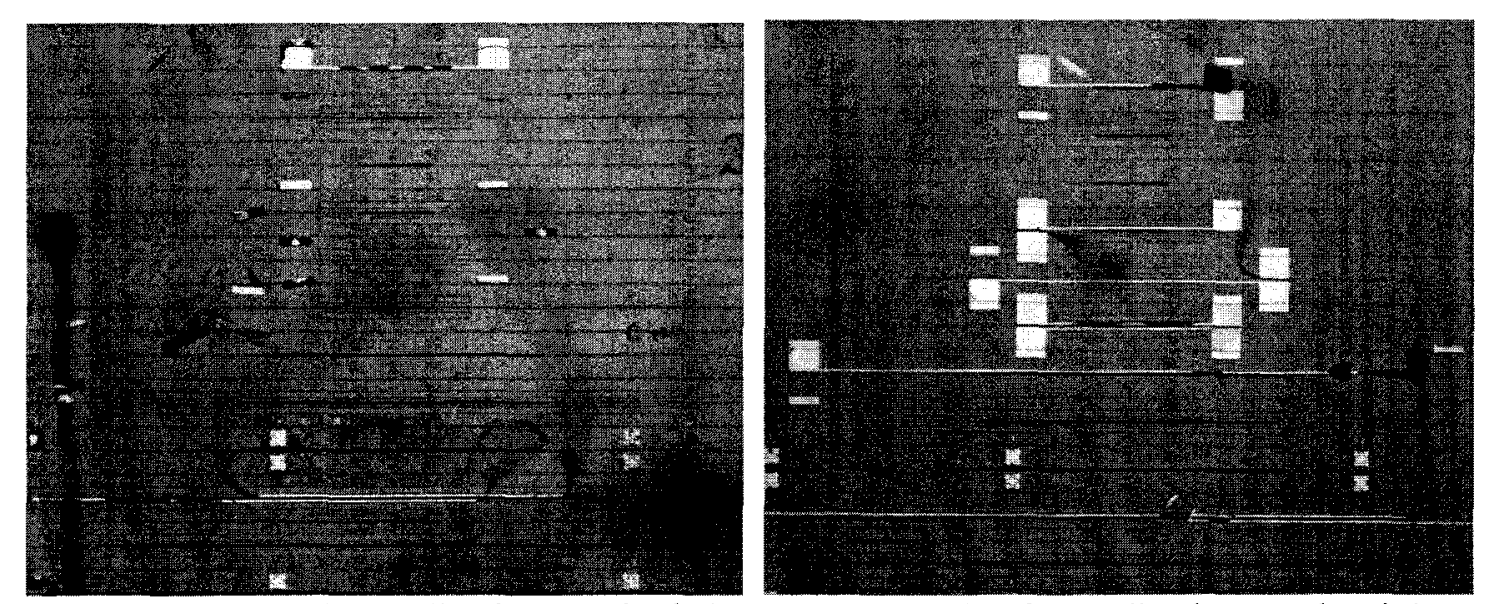

Figure 0.4. Chromium adhesion on the left (D2-30) and titanium adhesion on the right (D2-19) after the $\mathrm{PR}$ passivation layer was removed via $\mathrm{O}_{2}$ plasma ashing.

Post bridge release inspection from the SEM revealed an additional problem with step coverage from the gold heaters. The bond pads are $100 \mu \mathrm{m}$ square and at this size they overlap every other waveguide in the waveguide mask. These waveguides were not 
used optically on the die. The spacing is a hold over from the original waveguide mask design.

When the gold was evaporated, it condensed along the base of the rib and on top of the rib itself until (depending of the rib height) the top of one gold film made contact with the bottom of the other gold film. Because this is an evaporative process in which the samples were not rotated during the deposition, a cavity formed as can be seen in figure 0.5 and figure 0.6. This may have been another reason for delamination of the gold heaters. HF would penetrate into this cavity during the bridge release and this would help cause the metal to delaminate. Once the pad started to lift along the line defined by the waveguide, the entire heater would eventually delaminate.
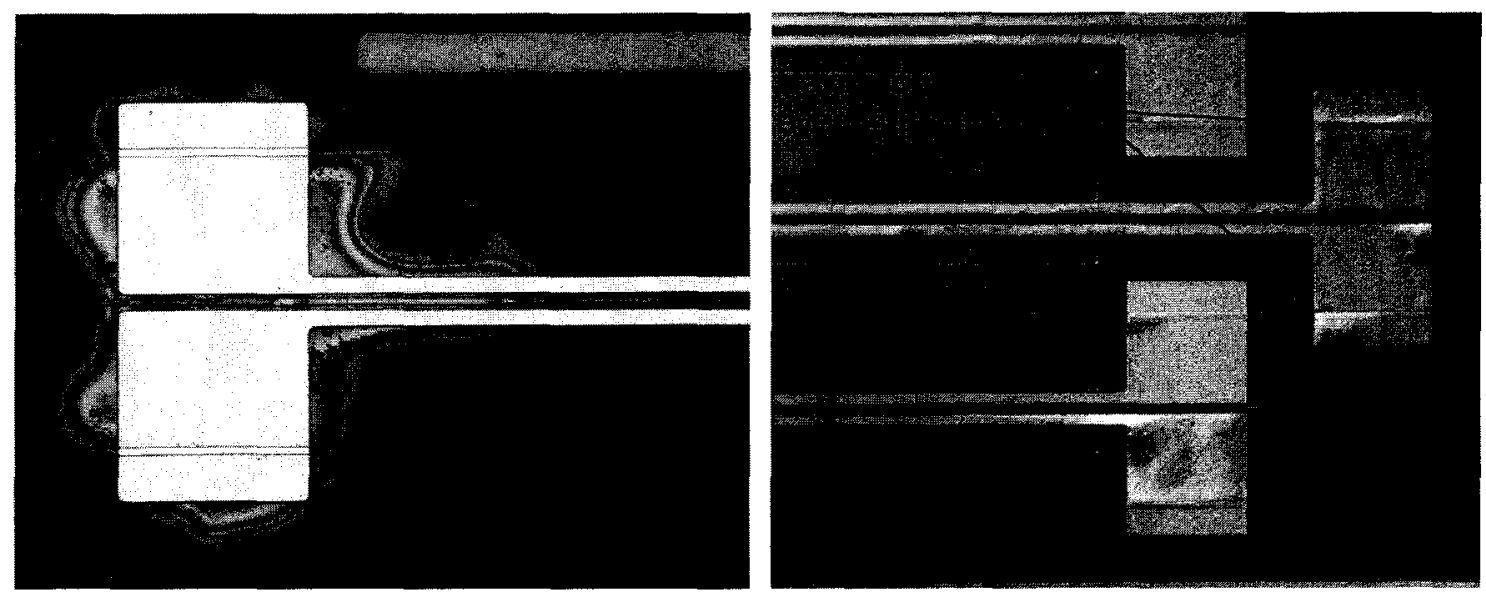

Figure 0.5 . D2-19 on the left shows where the photoresist passivation has failed after the HF bridge release. D2-8 on the right shows areas of failure along the step height differential after the HF bridge release etch and PR passivation removal.

Of the six die processed with PR passivation, only one has a successful bridge release with intact heater. The bond pad of this $500 \mu \mathrm{m}$ bridge device did not hold up to the wire bonder wedge. The SEM of the released device is shown in Figure 0.7. 

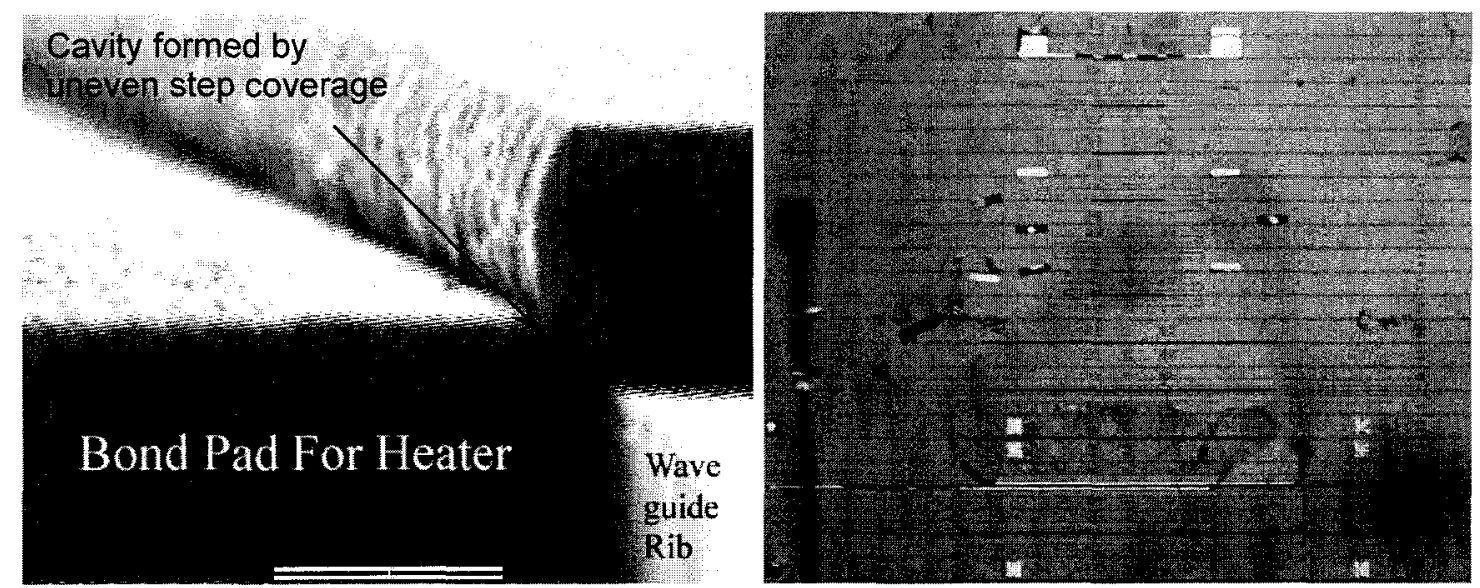

Figure 0.6. Example of step height coverage problem with D2-30 along with HF release.
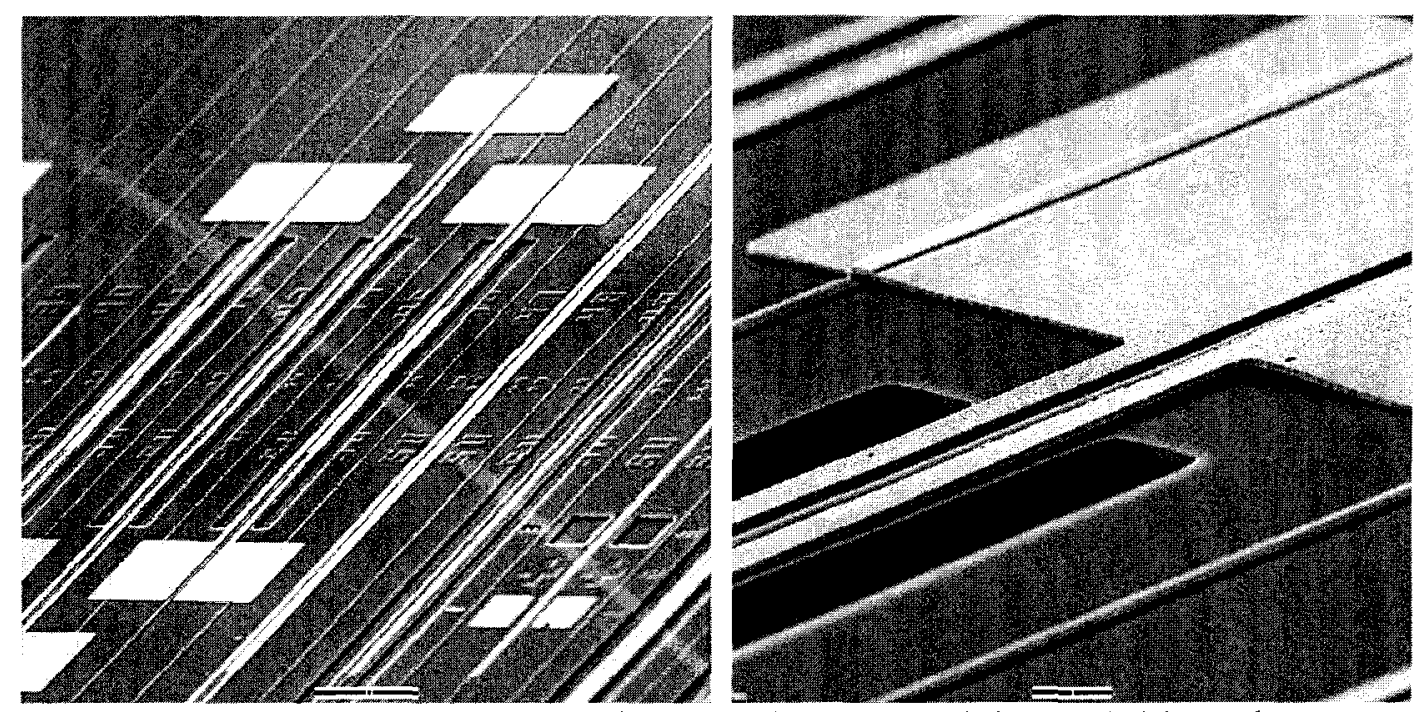

Figure 0.7 . D2-8 heaters on the 500 micron bridge survived the HF bridge release process though the heaters could not be successfully addressed with either probe tips or wire bonding. 


\section{References}

[1] R. Fearing, "Micro Structures and Micro Actuators for Implementing Sub-millimeter Robots," Precision Sensors, Actuators and Systems, Kluwer Academic Publishers, pp. 39-72 (1992).

[2] Y. Gianchandani and K. Najafi, "Batch Fabrication and Assembly of MicromotorDriven Mechanisms with Multi-Level Linkages," MEMS '92, pp. 141-146, (1992).

[3] C.-J Kim, A. Pisano, R. Muller, "Silicon-processed overhanging microgripper," J. MEMS, Vol. 1 No. 1, pp. 31-36 (1992).

[4] J. Sampsell, "The Digital Micromirror Device and its Application to Projection Displays," Transducers '93, pp. 24-27 (1993).

[5] K. Petersen, A. Shartel, and N. Raley, "Micromechanical accelerometer integrated with MOS detection circuitry," IEEE Trans. Electron. Dev., Vol. 29, pp. $23-27$ (1982).

[6] M. Schmidt, R. Howe, S. Senturia, and J. Haritonidis, "Design and calibration of a microfabricated floating-element shear stress sensor," IEEE Trans. Electron. Dev., Vol. 35 , pp. $750-757$ (1988).

[7] J. Yon, A. Astier, S. Bisotto, G. Chamingis, A. Durand, J. Martin, E. Mottin, J. Ouvrier-Buffet, and J. Tissot, "First demonstration of $25 \mu \mathrm{m}$ pitch uncooled amorphous silicon microbolometer IRFPA at LETI-LIR," Infrared Technology and Applications XXXI, Proceedings of SPIE 5783, pp. $432-440$ (2005).

[8] J. Walker, K. Goossen, and S. Arney, "Fabrication of a mechanical antireflection switch for fiber-to-the-home systems," J. MEMS, Vol. 5, No. 1, pp. 45-51 (1996).

[9] M. Motamedi, MOEMS: micro-opto-electro-mechanical systems, SPIE, (2005).

[10] M. Elwenspoek and H. Jansen, Silicon Micromachining, Cambridge University Press, 1998.

[11] Mechanical testing of silicon micro beam at Uppsala University, Division of Micro Systems Technology.

[12] C. Raum, Process characterization of (110) aligned silicon for Zenastra Photonics, Inc. (2001).

[13] P. Van Kessel, L. Hornbeck, R. Meier and M. Douglas, "A MEMS-based projection display," Proc. IEEE Vol. 86, pp. 1687-1704 (1998).

[14] H. Weinberg, "Dual axis, low g, fully integrated accelerometers," Analog Dialogue 33-1 (1999). 
[15] E. Palik, Handbook of Optical Constants of Solids, Academic Press N.Y., 1985.

[16] C. Marxer and N.F. de Rooij, "Micro-optomechanical $2 \times 2$ switch for single-mode fibers based on a plasma-etched silicon mirror and electrostatic actuation", Journal of Lightwave Technology, Vol. 17, No.1, pp. 2-6 (1999).

[17] M. Taher and A. Saif, "On a tunable bistable MEMS - theory and experiment," J. MEMS, Vol. 9 No. 2, pp. 157-170 (2000).

[18] M. Sinclair, "A High Frequency Resonant Scanner Using Thermal Actuation," Microsoft Research, Microsoft, Redmond, WA, 98052.

[19] A. Michael, MEMS Micro-bridge Actuator for Potential Applications in Optical Switching, Ph.D. Thesis, University of New South Wales, 2007.

[20] T. Bakke, C. Tigges, J. Lean, C. Sullivan and O. Spahn, "Planar Microoptomechanical Waveguide Switches," IEEE J. Sel. Topics In Quantum Electronics, Vol. 8, No. 1, pp. 64-72 (2002).

[21] M. Li, W. Yuen, G. Li, and C. Chang-Hasnain, "Top-emitting micromechanical VCSEL with a $31.6 \mathrm{~nm}$ tuning range," IEEE Photon. Tech. Lett., Vol. 10, No. 1, pp. 1820 (1998).

[22] M. Lafoy, 3D graphic art of suspended waveguide filter in post-buckle mode, based on design by Christopher Raum, Screen Images Ltd., Toronto, Ontario, Canada.

[23] A. Iocco, H. Limberger, R Salathé, L. Everall, K. Chisholm, J. Williams, and I. Bennion, "Bragg Grating Fast Tunable Filter for Wavelength Division Multiplexing," J. Lwv. Tech., 17 (7), pp. 1217-1221 (1999).

[24] A. Iocco, H. Limberger, R. Salathé, "Bragg grating fast tunable filter," Elec. Lett. 33 (25), pp. 2147-2148, (1997).

[25] H. Gnewuch, N. Zayer, C. N. Pannell, "Broadband monolithic acousto-optic tunable filter," Optics Letters, Vol. 25, No. 5, 2000.

[26] F. Arecco, D. Scarano, S. Schmid, D. Blumenthal, and R. Gaudino "Acousto Optic Devices In Add/Drop Multiplexer Nodes," ECOC98, 20-24 September 1998, Madrid, Spain.

[27] G. Kovacs, Micromachined Transducers Sourcebook, Ch. 6, McGraw Hill, 1998.

[28] K. Goossen, "MEMS-Based Variable Optical Interface Device," Optical MEMS, IEEE/LEOS International Conference 2000, pp. 17-18. 
[29] C. Madsen, J. Walker, J. Ford, K. Goossen, T. Nielsen, G. Lenz, "A Tunable Dispersion Compensating MEMS All-Pass Filter," IEEE Photonics Technology Letters, Vol. 12, No. 6, June 2000.

[30] R. Apte, F. Sandejas, W. Banyai and D. Bloom, "Grating Light Valves for High Resolution Displays," Solid State Sensors and Actuators Workshop, June 1994.

[31] Carlos Mateus, "Widely Tunable Torsional Optical Filter," IEEE Photonics Technology Letters, Vol. 14, No. 6, June 2002.

[32] R. Le Dantec, T. Benyattou, G. Guillot, A. Spisser, J. L. Leclercq, P. Viktorovitch, D. Rondi, R. Blondeau, "Tunable microcavity based on InP/air Bragg Mirrors," Journal Of Materials Science: Materials In Electronics, 10 (1999) pp. 447 - 450.

[33] J. Ford, V. Aksyuk, D. Bishop, J. Walker, "Wavelength Add-Drop Switching Using Tilting Micro-mirrors," Journal of Lightwave Technology, Vol. 17, No. 5, May 1999.

[34] D. Bishop, C. Giles, and G. Austin, "The Lucent Lambda Router: MEMS Technology of the Future Here Today," IEEE Communications Magazine, March 2002, pp. 75-79.

[35] T. Ohnstein, J. Zook, H. French, H. Guckel, T. Earles, J. Klein, P. Mangat, "Tunable IR Filters with Integral Electromagnetic Actuators," Solid-State Sensors and Actuator Workshop, Hilton Head, S.C., June 1996.

[36] T. Ohnstein, J. Zook, J. Cox, B. Speldrich, T. Wagener, H. Guckel, T. Christenson, J. Klein, T. Earles, and I. Glasgow, "Tunable IR filters using flexible metallic microstructures.” IEEE Proceedings: MEMS '95, pp. 170-174, (1995).

[37] S. Jin, R. Espindola, H. Mavoori, T. Strasser, J. DeMarco, "Magnetically Programmable Fibre Bragg Gratings," Electronics Letters, Vol. 34, No. 22, pp. 2158$2159,(1998)$.

[38] K. Lee and S. Lee, "Millimetre-sized Shape Memory Alloy Bending Actuator Using Buckling of the Beam," International Symposium on Micromechatronics and Human Science, pp 211-217 (1997).

[39] P. Surbled, B. Le Pioufle, E. Yang, H. Fujita, "Shape memory alloys for micromembranes actuation," Europto Series (EOS/SPIE symposia), Munich (D), 1999.

[40] S. Nagaoka, N. Take, Y. Tawa, Y. Ohba, Y. Takahashi, "Compact tunable FBG filters using Ni film heater and surface memory alloy (SMA) coil actuator," Lasers and Electro-Optics, 2001, Volume: 2, pp. II-26 - II-27, July 2001. 
[41] Louay Eldada, "Advances in telecom and datacom optical components," Opt. Eng. Vol. 40 No. 7 , pp 1165-1178, July 2001.

[42] D. Hohlfeld and H Zappe, "A Silicon Based Tunable Optical Filter Designed for Low Power Consumption and Fast Thermal Modulation," IEEE/LEOS International Conference on Optical MEMS, pp 171-172, 20-23 August 2002.

[43] G. Blau, M. Gale, J. Söchtig, C. Zschokke, R. Hövel, K. Gulden, "Tunable VCSEL with Integrated Heater and Planar Waveguide Wavelength Monitor," Proc. Opt. MEMS'00, Kauai, HI, Aug. 27-31, 2000, pp. 83-84.

[44] D. Hohlfeld, M. Epmeier, H. Zappe, "Tunable Thermo-optic Filter for WDM Applications," $15^{\text {th }}$ IEEE International Conference on MEMS, pp. 564-7 (2002).

[45] M. Oh, H. Lee, M. Lee, J. Ahn, S. Han, H. Kim, "Thermo-optic Tunable Wavelength Filters Using Polymer Waveguide Bragg Gratings," IEEE/LEOS Annual Meeting, Vol. 2, pp. 7-8 (1998).

[46] S. Yun and J. Lee, "A Micromachined In-Plane Tunable Optical Filter Using the Thermo-optic Effect of Crystalline Silicon," Journal of Micromechanics and Microengineering," Vol. 13, pp.721-725 (2003).

[47] L. Eldada, R. Blomquist, M. Maxfield, D. Pant, G. Boudoughian, C. Poga, R. Norwood, "Thermooptic Planar Polymer Bragg Grating OADM's with Broad Tuning Range," IEEE Photonics Technology Letters, Vol. 11, No. 4 (1999).

[48] X. Zhang and A. Liu, "A MEMS Pitch-tunable Grating Add/Drop Multiplexers," Optical MEMS 2000, IEEE and LEOS, Kauai, Hawaii, pp. 21-24 August 2000.

[49] T. Amano, F. Koyama, N. Nishiyama, K. Iga, "Temperature-Insensitive Micromachined AlGaAs-GaAs Vertical Cavity Filter," IEEE Photonics Technology Letters, Vol. 12, No. 5 (2000).

[50] R. Soref and B. Bennett, "Electro-optical Effects in Silicon," IEEE Journal of Quantum Electronics, Vol. 23, No. 123 (1987).

[51] R. Hunsperger, Integrated Optics: Theory and Technology, Springer-Verlag (2002).

[52] K. lizuka, Elements of Photonics, Volume II: For Fiber and Integrated Optics, Ch. 10, John Wiley \& Sons, Inc. (2002).

[53] A. Marcatili, "Dielectric rectangular waveguide and directional coupler for integrated optics," Bell Systems Technical Journal, Vol. 48, pp. 2071-2102 (1969). 
[54] H. Furuta, H. Noda, A. Ihaya, "Novel Optical Waveguide for Integrated Optics," Applied Optic, Vol. 13, pp. 322-326 (1974).

[55] R. Soref, J. Schmidtchen, and K. Petermann, "Large Single-Mode Rib Waveguides in GeSi-Si and Si-on-SiO 2 ," Journal of Quantum Electronics, Vol. 27, No. 8, pp. 19711974 (1991).

[56] T. Tamir, Integrated Optics, Ch 2, Berlin: Springer-Verlag (1975).

[57] K. Ramdas, An Electronically-Tunable Bragg Grating in Silicon-On-Insulator, M.Sc. Thesis, Carleton University (2004).

[58] T. Tamir ed., Integrated Optics, H. Kogelnik, Ch 2, "Theory of Dielectric Waveguides," Springer-Verlag (1979).

[59] K. Okamoto, Fundamentals of Optical Waveguide, Ch 2, p. 38, Academic Press, San Diego (1992).

[60] A. Yariv, "Coupled mode theory for guided wave optics," J. Quan. Electron., Vol. QE-9, No. 9, pp. 919-933 (1973).

[61] A. Yariv and M. Nakamura, "Periodic Structures for Integrated Optics," J. Quan. Electron., Vol. QE-13, No. 4, pp. 233-253 (1977).

[62] W. Tropf and M. Thomas, "Infrared Refractive Index and Thermo-optic Coefficient Measurement at APL," John Hopkins APL Tech. Digest, Vol. 19, No. 3, pp. 293-298 (1998).

[63] M. Fox, Optical Properties of Solids, Oxford University Press (2001).

[64] F. Corte, M. Montefusco, L. Moretti, I. Rendina, G. Cocorullo, "Temperature dependence analysis of the thermo-optic effect in silicon by single and double oscillator models," J. Appl. Phys., Vol. 88, No. 12, pp. 7115-7119 (2000).

[65] Y. Okada, Y. Tokumaru, "Precise determination of lattice parameter and thermal expansion coefficient of silicon between 300 and 1500 K," J. Appl. Phys, Vol. 56, No. 2, pp. 314-320 (1984).

[66] R. Hull ed., Properties of Crystalline Silicon, The Institution of Electrical Engineers (1999).

[67] Properties of Silicon (INSPEC, London, 1988) EMIS Data Reviews Series, No. 4.

[68] U. Behner, Cubic hexoctahedral output from crystal growth Javascript, Zum Bildchen 62 - 66687 Wadern, Germany. 
[69] W. Brantley, "Calculated Elastic Constants for Stress Problems Associated with Semiconductor Devices," J. Appl. Phys., Vol. 44, pp. 534-535 (1973).

[70] J. Nye, Physical Properties of Crystals, Ch 13, Oxford Press (1957).

[71] A Yariv and P. Yeh, Optical Waves in Crystals, Ch 9, Wiley Interscience (1984).

[72] S. He, T. Zheng and S. Danyluk, "Analysis and Determination of the Stress-optic Coefficients of Thin Single Crystal Silicon Samples," J. Appl. Phys., Vol. 96, No. 6, pp. 3103-3109 (2004).

[73] A. Giardini, "Piezobirefringence in Silicon," American Mineralogist, Vol. 43, pp. 249-262 (1958).

[74] R. Craig, Mechanics of Materials, Ch 6, 7 and 10, John Wiley \& Sons (2000).

[75] A. Usmani and J. Rotter, "Fundamental Principles of Structural Behaviour Under Thermal Effects," Fire Safety Journal, Vol. 36, pp. 721-744 (2001).

[76] J. Qiu, J. Lang, A. Slocum, "A Curved-Beam Bistable Mechanism," J. MEMS, Vol. 13, No. 2, pp. 137-146 (2004).

[77] A. Michael, C. Kwok, "Design Criteria for Bi-stable Behaviour in a Buckled Multilayered MEMS Bridge," J. Micromech. And Microeng., Vol. 16, pp. 2034-2043 (2006).

[78] M. Vangbo, "An analytical analysis of a compressed bistable buckled beam," Sensors and Actuators A, Vol. 69, pp. 212-216 (1998).

[79] S. Senturia, Microsystem Design, Springer (2001).

[80] W. Pilkey, Formulas for Stress, Strain, and Structural Matrices, Wiley (1994).

[81] J. Zahn, N. Talbot, D. Liepmann, A. Pisano, "Microfabricated Polysilicon Microneedles for Minimally Invasive Biomedical Devices," Biomedical Microdevices Vol. 2, No. 4, pp. 295-303 (2000).

[82] W. Fang and J Wickert, "Post buckling of micromachined beams," J. Micromech. Microeng. Vol. 4, pp. 116-122 (1994).

[83] M. Saif and N. MacDonald, “A Millinewton Microloading Device," Sensors and Actuators A, Vol. 52, pp 65-75 (1996).

[84] M. Saif and N. MacDonald, "Measurement of Forces and Spring Constants of Microinstruments," Rev. of Sci. Instruments, Vol. 69, No. 3, pp. 1410-1422 (1998). 
[85] J. Stewart, Calculus - Early Transcendentals, Brooks/Cole (1991).

[86] R. Arya, M Rashid, D. Howard, S. Collnis, R. Smith, "Thermally Actuated, Bistable, Snapping Silicon Membranes," $12^{\text {th }}$ International Conference on Solid State Sensors, Actuators and Microsystems (2003).

[87] S. Pogossian, L. Vescan, A. Vonsovici, "The Single-Mode Condition for Semiconductor Rib Waveguides with Large Cross Section," J. of Lightwave Tech., Vol. 16, No. 10, pp. 1851-1853 (1998).

[88] A. Rickman, G. Reed and F. Namavar, "Silicon-On-Insulator Optical Rib Waveguide Loss and Mode Characteristics," J. Lightwave Technol., Vol. 12, pp. 17711776 (1994).

[89] Apollo Photonic Solutions Suite, Apollo Photonics Inc., Hamilton, Ontario, Canada.

[90] Maissel and Glang, Handbook of Thin Film Technology, Ch 3, "Thin Film Resistors," McGraw-Hill (1970).

[91] J. Stanley, Electrical and Magnetic Properties of Metals, American Society for Metals, Metals Park, OH (1963).

[92] A. Othonos and K. Kalli, Fiber Bragg Gratings - Fundamentals and Applications in Telecommunications and Sensing, Artech House (1999).

[93] C. Raum, Fibre Optic Alignment Using Micromachines, Masters Thesis, University of Regina (1997).

[94] S. Timoshenko and J. Gere, Mechanics of Materials, Ch. 5, "Stresses in Beams," Van Nostrand Reinhold Ltd. (1972).

[95] K. Mnaymneh, Photonic Quasicrystals: A Case For Crystal Angular Momentum, Ph.D. Thesis, Carleton University (2007).

[96] J. Plummer, M. Deal, P. Griffin, Silicon VLSI Technology, Prentice Hall (2000).

[97] HTE Labs, "SiO2 colour chart for thermally grown silicon dioxide," Santa Clara, California, USA.

[98] Maissel and Glang, Handbook of Thin Film Technology, Ch. 12, "Mechanical Properties of Thin Film," McGraw-Hill (1970).

[99] K. Williams and R. Muller, "Etch Rates for Micromachining Processes," J. MEMS Vol. 5, No. 4, pp. 256-269 (1996). 
[100] K. Williams, K. Gupta, M. Wasilik, "Etch Rates for Micromachining Processes Part II," J. MEMS, Vol. 12, No. 6, pp. 761-778 (2003).

[101] C. Raum, "Crystallographic properties of silicon relating to the techniques of preparing a waveguide facet," portion of a short course on silicon waveguide fabrication processes, Carleton University, Feb 26 and 27, 2007 and Feb 25 and 26, 2008.

[102] J. Bühler, F-P. Steiner, and H. Baltes, "Silicon dioxide sacrificial layer etching in surface micromachining," J. Micromech. Microeng. Vol. 7, pp. R1-R13 (1997).

[103] N. Tas, T. Sonnenberg, H. Jansen, R. Legtenberg, and M. Elwenspoek, "Stiction in surface micromachining," J. Micromech. Microeng. Vol. 6, pp. 385-397 (1996).

[104] J. Rose, L. Roy, and N. Tait, "Development of a MEMS microwave switch and application to adaptive integrated antennas," IEEE CCECE 2003, Vol.3, pp. 1901- 1904 May 2003.

[105] G. Kovaks, Micromachined Transducers Sourcebook, McGraw-Hill (1998).

[106] J. Kiihamäki, "Measurement of oxide etch rate of SOI structure using near IR microscopy," Physica Scripta, Vol. T101, pp. 185-187 (2002).

[107] T. Abe, W. Messner and M. Reed, "Effects of Elevated Temperature Treatments in Microstructure Release Procedures," J. MEMS, Vol. 4, pp. 66-75 (1995).

[108] N. Tien, S. Jeong, L. Phinney, K. Fushinobu, and J. Boker, "Surface Adhesion Reduction in Silicon Microstructures using Femtosecond Laser Pulses," Appl. Phys. Lett., Vol. 68, pp. 197-199 (1996).

[109] A. Kovacs and A. Stoffel, "Process Optimization Of Free-Standing Polysilicon Microstructures," J. Micromech. Microeng. Vol. 2, pp. 190-192 (1992).

[110] Shom S. Ponoth, Navnit T. Agarwal, Peter D. Persans, Joel L. Plawsky, "Fabrication of controlled sidewall angles in thin films using isotropic etches," J. Vac. Sci. Technol. B Vol. 21, No. 4, pp. 1240-1247 (2003).

[111] T. Yanagawa and I. Takekoshi, "Failure Analysis of Evaporated Metal Interconnections at Contact Windows," IEEE Trans. Electron. Devices, Vol. 17, No. 11, pp. 964-970 (1970).

[112] J. North, T. McGahan, D. Rice and A. Adams, "Tapered Windows in PhosphorusDoped SiO2 by Ion Implantation," IEEE Trans. Electron. Dev. Vol. 25, No. 7, pp. 809812 (1978). 
[113] Y. Choi, Y-Se Kwon, and C-Ki Kim, "Graded Etching of Thermal Oxide with Various Angles using Silica film," IEEE Electron. Dev. Letters, Vol. 1, No. 3, pp. 30-31 (1980).

[114] Jyrki Kiihamäki, Fabrication of SOI Micromechanical Devices, Ph.D. Thesis, VTT Technical Research Centre of Finland (2005).

[115] T. Murphy, J. Hastings, and H. Smith, "Fabrication and Characterization of Narrow-Band Bragg-Reflection Filters in Silicon-on-Insulator Ridge Waveguides," J. Lightwave Tech., Vol. 19, No. 12, pp. 1938-1942 (2001).

[116] I. Giuntoni, M. Krause, H. Renner, J. Bruns, A. Gajda, E. Brinkmeyer, and K. Petermann, "Numerical Survey on Bragg Reflectors in Silicon-on-Insulator Waveguides," 5th Int. Conf. on Group IV Photonics (IEEE/LEOS 2008), pp. 285-287.

[117] I. Giuntoni, A. Gajda, M. Krause, R. Steingruber, J. Bruns, K. Petermann, "Tunable Bragg reflectors on silicon-on-insulator rib waveguides," Optics Express, vol. 17, no. 21 (2009).

[118] S. Homampour, M. Bulk, P. Jessop, and A. Knights, "Thermal tuning of planar Bragg gratings in silicon-on-insulator rib waveguides," Phys. Status Solidi C 6, No. S1, S240-S243 (2009).

[119] C. Chen and J. Albert, "Strain-optic coefficients of individual cladding modes of single mode fibre: theory and experiment," Electronics Letters, Vol. 42, No. 18 (2006).

[120] C. Raum, R. Tait, and R. Gauthier, "Fabrication and characterization of a thermomechanically tunable grating-assisted suspended waveguide filter," Proceedings of SPIE, Vol. 6898, pp. 68981E-1 - 9 (2008).

[121] A. Enianga, B. Obiesioa, K. Akpana, and C. Raum, "Fabrication and characterization of an SOI based thermally tuned phase modulator," Proc. of SPIE, Vol. $6796(2007)$

[122] C. Raum, R. Tait, and R. Gauthier, "Controlled sacrificial sidewall surface micromachining for the release of high aspect ratio bridges," J. Vac. Sci. Technol. B, accepted (2010).

[123] C. Raum, R. Gauthier, and N. Tait, "Analysis of a Bistable Thermally Tunable Waveguide," Proc. of SPIE, Vol. 5970, pp. 356-366, (2005).

[124] C. Raum, R. Gauthier, and R. Tait, "Fabrication and characterization of a thermally tunable Bragg filter on a suspended SOI waveguide", SPIE Great Lakes Photonics Symposium, 2006. 
[125] C. Raum, R. Gauthier, and N. Tait, "Integrated heaters for the thermal tuning of Bragg filters on silicon-on-insulator rib.waveguides," Microw. Opt. Techn. Let., submitted (2010).

[126] M. Roberts, L. Klein, D. Savage, K. Slinker, M. Friesen, G. Celler, M. Eriksson, and M. Lagally, "Elastically relaxed free-standing strained-silicon nanomembranes," Nature Materials, Vol. 5, pp. 388-393 (2006).

[127] M. Chiao and L. Lin, "Self-Buckling of Micromachined Beams Under Resistive Heating," J. MEMS, Vol. 9, pp. 146-151 (2000). 